\title{
Catalytic Enantioselective Syn-Hydroxy-Oxyacylation of Electron Deficient Alkenes
}

Chandra Bhan Pandey, Vikram Singh, Susanta Ghanta, Bhoopendra Twari

Submitted date: 01/04/2021 Posted date: 01/04/2021

Licence: CC BY-NC-ND 4.0

Citation information: Pandey, Chandra Bhan; Singh, Vikram; Ghanta, Susanta; Twari, Bhoopendra (2021):

Catalytic Enantioselective Syn-Hydroxy-Oxyacylation of Electron Deficient Alkenes. ChemRxiv. Preprint. https://doi.org/10.26434/chemrxiv.14345981.v2

Asymmetric syn-dihydroxylation and dioxyacylation of alkenes have been well established. A direct method for the enantioselective preparation of orthogonally protected syn-1,2-diols from alkenes is unprecedented. Here in, we report the first enantioselective hypervalent iodine catalyzed syn-hydroxy-oxyacylation of enones. The orthogonally protected diols were obtained with excellent diastereo- and regioselectivity under metal-free condition. For these electron-deficient alkenes, even the syn-dihydroxylation and dioxyacylation have remained yet an unfinished challenge.

File list (2)

CBP_CA_fv.pdf $(722.35 \mathrm{KiB})$

view on ChemRxiv - download file 


\title{
Catalytic Enantioselective syn-Hydroxy-Oxyacylation of Electron Deficient Alkenes
}

\author{
Chandra Bhan Pandey, ${ }^{\dagger}$ Vikram Singh,${ }^{\dagger}$ Susanta Ghanta,${ }^{\ddagger}$ and Bhoopendra Tiwari ${ }^{\dagger *}$ \\ 'Division of Molecular Synthesis \& Drug Discovery, Centre of Biomedical Research, SGPGIMS-Campus, Raebareli Road, \\ Lucknow, 226014, India. \\ Department of Chemistry, National Institute of Technology, Agartala, 799046, Tripura, India.
}

This paper is dedicated to late Professor Kilian Muniz

\begin{abstract}
Asymmetric syn-dihydroxylation and dioxyacylation of alkenes have been well established. A direct method for the enantioselective preparation of orthogonally protected $s y n$-1,2-diols from alkenes is unprecedented. Here in, we report the first enantioselective hypervalent iodine catalyzed syn-hydroxy-oxyacylation of enones. The orthogonally protected diols were obtained with excellent diastereo- and regioselectivity under metal-free condition. For these electron-deficient alkenes, even the syn-dihydroxylation and dioxyacylation have remained yet an unfinished challenge.
\end{abstract}

Orthogonally protected diols and triols are privileged structural motifs in numerous biologically active natural products, pharmaceutics, functionalized materials, and are important intermediates in many syntheses. ${ }^{1}$ Therefore, numerous synthetic approaches have been developed to stereoselectively install double syn $\mathrm{C}-\mathrm{O}$ bonds on alkenes (Scheme 1). The oxidative dihydroxylation of alkenes catalyzed by $\mathrm{OsO}_{4}$, especially Sharpless dihydroxylation, has emerged as an extremely reliable variant. ${ }^{2}$ However, the high toxicity, expensiveness and volatility of this catalyst led to the renewed interest in developing catalysts based on other transition metals, such as ruthenium, ${ }^{3}$ palladium, ${ }^{4}$ manganese ${ }^{5}$ and iron. ${ }^{6}$ The metalfree methods due to their intrinsic properties have also generated a great interest using peroxides, ${ }^{7}$ hydroxamic acids,${ }^{8}$ hypervalent iodine reagents, ${ }^{9}$ organocatalysts ${ }^{10}$ and biocatalysts. ${ }^{11}$ The group of Fujita reported the first asymmetric variant of Prevost and Woodward method for the dihydroxylation using chiral hypervalent iodine reagent in $2011 .^{12}$ Besides dihydroxylation, asymmetric diacetoxylation ${ }^{13}$ and dialkoxylation ${ }^{14}$ have also garnered significant importance for the asymmetric functionalization. However, for any effective direct chemoselective transformation, the difunctionalities are ideally desired to be in the differentiated/orthogonally protected form to avoid unproductive protection and deprotection steps in a multistep synthesis.

Enones are an important class of olefins which act as the surrogates for accessing triols after deoxygenation, followed by a selective reduction. It has surprisingly remained beyond the scope of almost all the known methods for enantioselective syn-dihydroxylation or diacylation. In 2002, the group of Brown pioneered the 1,2dihydroxylation of enones using a phase transfer catalyst in a moderate yield of $19-52 \%$ and $60-80 \%$ ee (Scheme 1a). ${ }^{10 \mathrm{~b}}$ This method is compatible with enones

\section{Scheme 1. Asymmetric Hydroxy-Oxyacetylation of Enones}

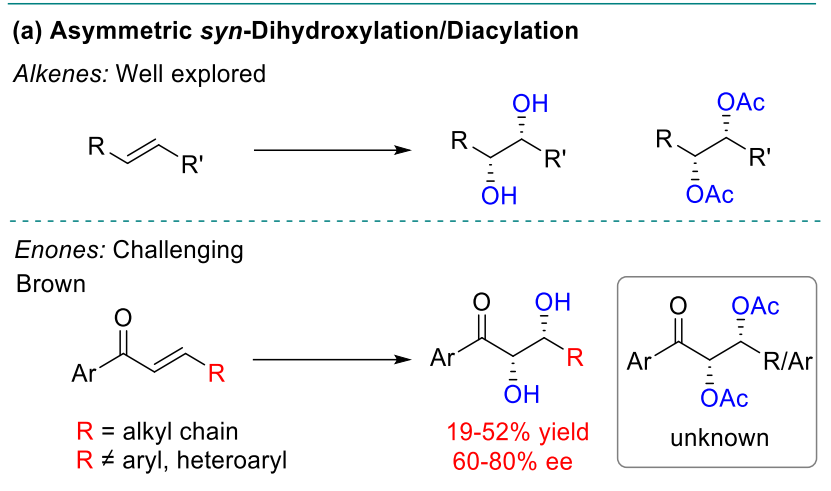

(b) Asymmetric syn-Hydroxy-Oxyacylation: Not Known

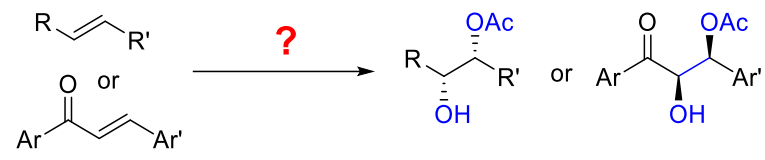

This Work: Enantioselective syn-Hydroxy-Oxyacylation of Enones

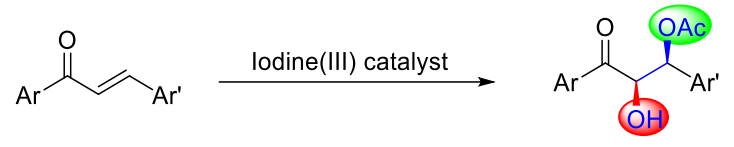

up to $96 \%$ yield and $99 \%$ ee

substituted with less bulky alkyl chains at the $\beta$-position, and the sterically demanding (hetero)aryls and polycyclic aryls are beyond its scope. To the best of our knowledge, (i) there is no report on asymmetric syn-hydroxy-oxyacylation of electron-deficient alkenes, and (ii) for enones, even the syn-dihydroxylation with a high enantioselectivity has remained unfinished task, whereas the dioxyacylation is yet to be reported (Scheme 1b). Herein, we describe the first highly enantio and regioselective syn-1,2-hydroxy-oxyacylation of enones using hypervalent iodine catalyst (Scheme 1b). Acetic acid/ $\mathrm{H}_{2} \mathrm{O}$ (residual water from the reagents) act as the source of acyloxy and hydroxy groups.

Our initial investigation focused on identifying a suitable hypervalent iodine catalyst, generated in situ, for the asymmetric 
Table 1. Reaction Condition Optimization ${ }^{a}$

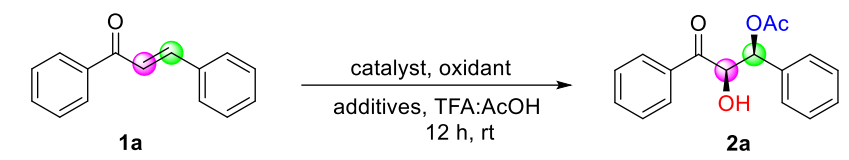

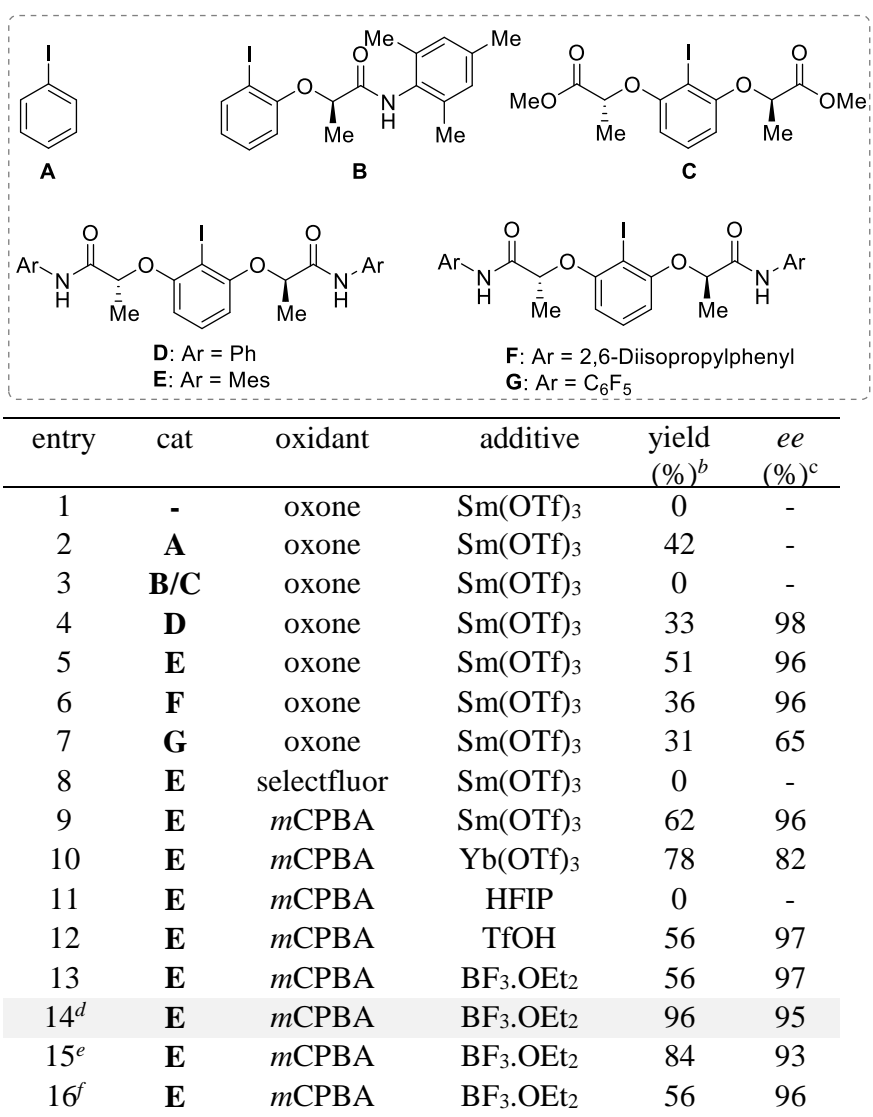

${ }^{a}$ Reaction condition unless otherwise specified: 1a $(0.1 \mathrm{mmol})$, additive (20 mol \%), I(III) catalyst A-G (20 mol \%), oxidant (1.2 equiv.), solvent ( $2.0 \mathrm{~mL}, 1: 1$ ratio) at $\mathrm{rt}^{b}{ }^{b}$ Isolated yield. ${ }^{c}$ Determined by HPLC analysis on chiral stationary phase. ${ }^{d} 50 \mathrm{~mol} \%$ of $\mathrm{BF}_{3}$. OEt 2 was used. ${ }^{e}$ Reaction performed at $0{ }^{\circ} \mathrm{C}$. ${ }^{f} 30 \mathrm{~mol} \%$ of catalyst loading was used.

syn-1,2-hydroxy-oxyacylation of chalcone 1a (Table 1). Various class of chiral aryl iodides (B-G) were tested as the potential catalysts in the presence of oxone in AcOH:TFA (1:1) as the solvent and $\mathrm{Sm}(\mathrm{OTf})_{3}$ as the additive. Initial breakthrough was observed in the presence of $20 \mathrm{~mol} \%$ of achiral catalyst A, giving the desired hydroxy-oxyacetylated product $\mathbf{2 a}$ as a single diastereomer in $42 \%$ yield (entries 1 and 2). Among the different catalysts screened, catalyst $\mathbf{E}$ provided 2a with a decent yield of $51 \%$ and $96 \%$ ee (entry 5 ). We next evaluated various oxidants. The use of selectfluor was found to be unsuitable while $m$ CPBA produced 2a in $62 \%$ yield and $96 \%$ ee (entries 8-9). The variation of Lewis/Bronsted acids showed a dramatic effect on the yield and $\mathrm{BF}_{3} . \mathrm{OEt}_{2}$ was the optimal choice giving the desired product in $96 \%$ yield with $95 \%$ ee (entry 14). The structure and the absolute configuration was unambiguously confirmed through X-ray crystallographic analysis of $\mathbf{2 d}$ (Scheme 2). ${ }^{15}$

With the optimized reaction condition in hand, chalcones bearing different electron-donating groups (EDG) as well as electron-withdrawing groups (EWG) at ortho, meta and para-position of the aryl rings were examined (Scheme 2). The EDGs
Scheme 2. Substrate scope

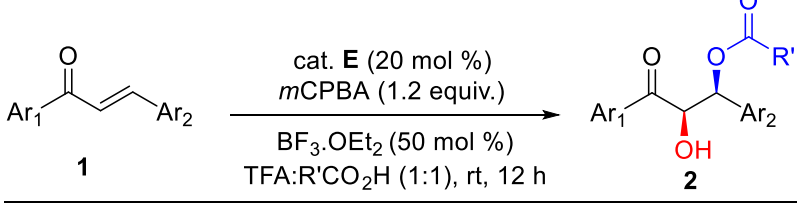<smiles>[R]#CC(=O)c1ccc(C(=O)C(O)c2ccccc2)cc1</smiles>

2a, $\mathrm{R}=\mathrm{H}, 96 \%, 95 \%$ ee 2b, $\mathrm{R}=4-\mathrm{Me}, 73 \%, 98 \%$ ee 2c, $\mathrm{R}=4-\mathrm{OMe}, 93 \%, 89 \%$ ee 2d, $\mathrm{R}=4-\mathrm{Br}, 76 \%, 96 \%$ ee 2e, $\mathrm{R}=4-\mathrm{Cl}, 64 \%, 96 \%$ ee 2f, $\mathrm{R}=4-\mathrm{NO}_{2}, 21 \%, 92 \%$ ee<smiles>[R][X]1cccc([C@H](OC(C)=O)[C@@H](O)C(=O)c2ccccc2)c1</smiles>

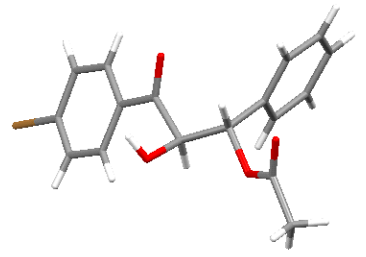

X-ray structure of $\mathbf{2 d}$<smiles>CC(=O)OC(c1ccc(C)cc1)C(O)C(=O)c1ccc(C)cc1</smiles>

2g, $\mathrm{R}=4-\mathrm{Me}, 68 \%, 83 \%$ ee 2h, R $=4-\mathrm{Cl}, 60 \%, 94 \%$ ee $2 \mathrm{i}, \mathrm{R}=2-\mathrm{Br}, 62 \%, 96 \%$ ee 2j, $R=4-B r, 65 \%, 93 \%$ ee 2k, R $=4-F, 72 \%, 92 \%$ ee

2I, $\mathrm{R}=4-\mathrm{Me}, 60 \%, 88 \%$ ee $2 \mathrm{~m}, \mathrm{R}=4$-Et, $69 \%, 91 \%$ ee 2n, $\mathrm{R}=4-\mathrm{Br}, 64 \%, 94 \%$ ee 2o, $\mathrm{R}=4-\mathrm{Cl}, 84 \%, 95 \%$ ee<smiles>[R]Pc1ccc(C(OC(C)=O)C(O)C(=O)c2ccc(Br)cc2)cc1</smiles>

2p, $\mathrm{R}=3-\mathrm{Me}, 68 \%, 98 \%$ ee 2q, $R=4-M e, 64 \%, 91 \%$ ee $2 r, R=4-E t, 81 \%, 94 \%$ ee 2s, $R=4{ }^{i} \operatorname{Pr}, 73 \%$, 96\% ee<smiles>CC(=O)OC(c1ccccc1)C(O)C(=O)c1cccs1</smiles>

$2 \mathrm{x}, 81 \%, 95 \%$ ee<smiles>CC(=O)O[C@H](c1ccccc1)[C@@H](O)C(=O)c1ccc(Cl)cc1</smiles>

2t, $\mathrm{R}=3-\mathrm{Me}, 81 \%, 99 \%$ ee 2u, $\mathrm{R}=4-\mathrm{Me}, 76 \%, 94 \%$ ee $2 v, R=4-E t, 64 \%, 94 \%$ ee $2 \mathbf{w}, \mathrm{R}=4{ }^{i} \mathrm{Pr}, 75 \%, 93 \%$ ee<smiles>CC(=O)OC(c1ccccc1)C(O)C(=O)c1ccc2ccccc2c1</smiles>

$2 y, 78 \%, 94 \%$ ee<smiles>CC(=O)OC(c1cccc2ccccc12)C(O)C(=O)c1ccccc1</smiles>

2z, $73 \%^{a}$<smiles>CCC(=O)OC(c1ccccc1)[C@H](O)C(=O)c1ccccc1</smiles>

2ab, $66 \%, 93 \%$ ee<smiles>CC(=O)OC(c1ccccc1)C(O)C(=O)/C=C/c1ccccc1</smiles>

2aa, $62 \%, 98 \%$ ee<smiles>CCCCC(=O)OC(c1ccccc1)C(O)C(=O)c1ccccc1</smiles>

2ac, $60 \%, 90 \%$ ee

${ }^{a}$ HPLC separation for $2 z$ was not achieved, see $S$ I for further details.

like $\mathrm{Me}, \mathrm{Et}, \mathrm{OMe},{ }^{i} \mathrm{Pr}$; and EWGs like flouro, chloro, bromo on the aryl ring $\mathrm{Ar}_{1}$ gave the desired products in good to excellent yields and high enantioselectivity. Substitution patterns on the $\beta$-aryl ring $\left(\mathrm{Ar}_{2}\right)$ were also compatible with the optimized reaction condition producing the desired products in good yields and excellent ee. ${ }^{16}$ Switching from acetic acid to higher analogues also furnished 2ab and $\mathbf{2 a c}$ in good yields without any loss in enantioselectivity. 


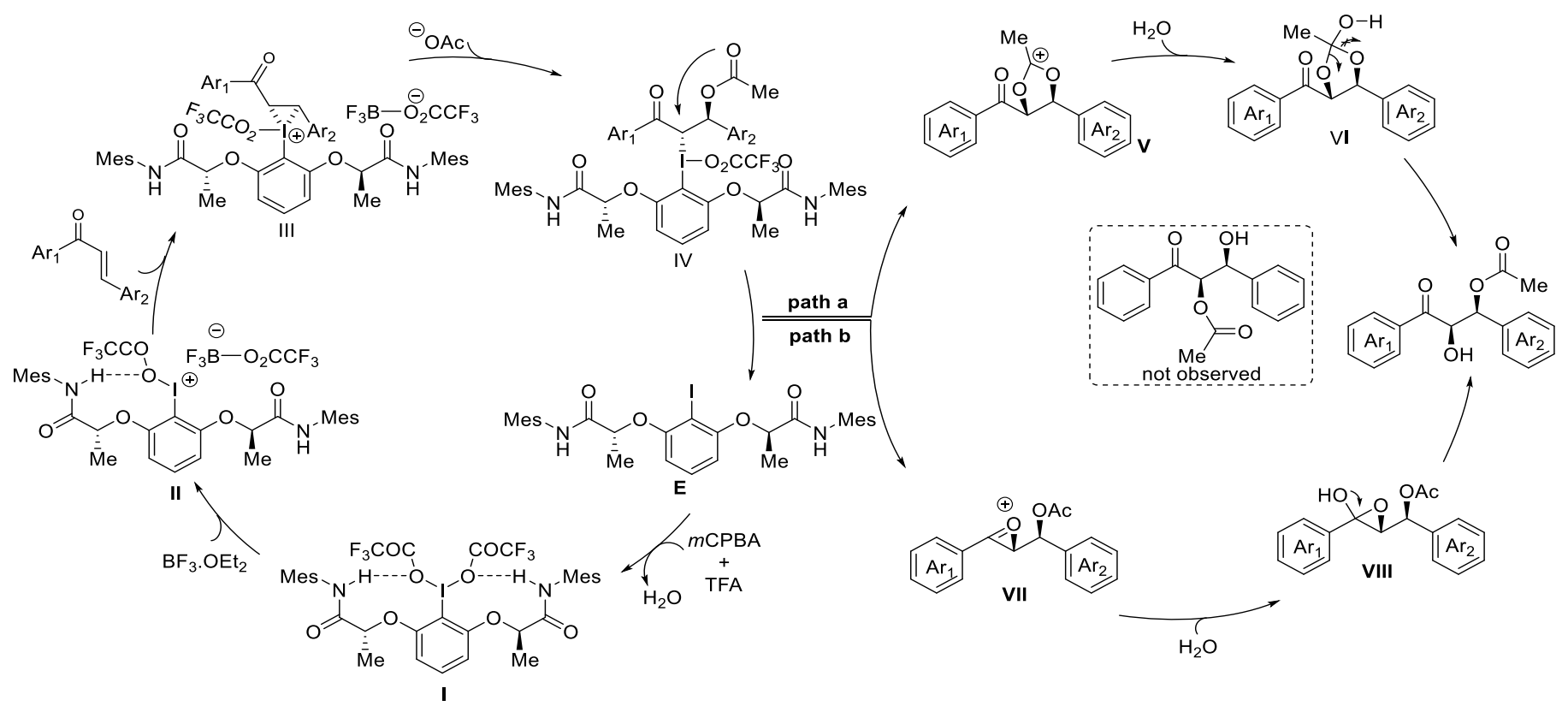

Our proposed reaction mechanism for this transformation is detailed in Scheme 3. The catalytic cycle begins with the in situ oxidative generation of I(III) in presence of $m \mathrm{CPBA} / \mathrm{TFA}$ that reacts with $\mathrm{BF}_{3} . \mathrm{OEt}_{2}$ to produce intermediate II. This intermediate coordinates with the chalcones $\mathbf{1}$ to give adduct III. A regioselective substitution by an acetate generates intermediate $\mathbf{I V}$. The regeneration of catalyst $\mathbf{E}$ takes place via reductive elimination to give either intermediate $\mathbf{V}$ (path a) or intermediate VII (path b). Subsequent regioselective hydrolysis of intermediate VI or VIII generates the desired product with excellent regio- and stereoselecvity. A detailed DFT calculation is underway to ascertain the preferred reaction pathway among the path a and path $b$.

In conclusion, we have achieved the first enantioselective synhydroxy-oxyacylation of electron deficient olefins using hypervalent iodine(III) catalysis. This method furnished the orthogonally protected syn-diols from alkenes with a wide substrate scope and excellent enantiocontrol. Further studies towards selective transformation of the products is under progress in our laboratory.

\section{ASSOCIATED CONTENT}

\section{Supporting Information}

Experimental procedure, characterization data and spectra (PDF)

\section{AUTHOR INFORMATION}

\section{Corresponding Author}

E-mail: btiwari@cbmr.res.in

\section{Notes}

The authors declare no competing financial interests.

\section{ACKNOWLEDGMENT}

C. B. P. thanks the University Grand Commission (UGC), New Delhi, India, for the fellowship. Financial support by SERB, New Delhi, India, (CRG/2018/004424) is gratefully acknowledged.

\section{REFERENCES}

1. (a) Edagwa, B. J.; Taylor, C. M. J. Org. Chem. 2009, 74, 4132. (b) Gardiner, J. M.; Panchal, N. R.; Stimpson, W. T.; Herbert, J. M.; Ellames, G. J. Synlett 2005, 2685. (c) Gancitano, P.; Ciriminna, R.; Testa, M. L.; Fidalgo, A.; Ilharco, L. M.; Pagliaro, M. Org. Biomol. Chem. 2005, 3, 2389. (d) Gupta, P.; Naidu, S. V.; Kumar, P.; Tetrahedron Lett. 2004, 45, 849. (e) Pye, P. J.; Rossen, K.; Weissman, S. A.; Maliakal, A.; Reamer, R. A.; Ball, R.; Tsou, N. N.; Volante, R. P.; Reider, P. J. Chem. Eur. J. 2002, 8, 1372. (f) Kang, S. H.; Jeong, J. W.; Hwang, Y. S.; Lee, S. B. Angew. Chem. Int. Ed. 2002, 41, 1392. (g) Ruiz, M; Ojea, V; Quintela, J. M. Tetrahedron: Asymmetry 2002, 123, 1535. (h) Johnson, R. A.; Sharpless, K. B. in Catalytic Asymmetric Synthesis, 2nd ed.; Ojima, I., Ed.; VCH: Weinheim, 2000, p 357. (i) Schultze, L. M.; Chapman, H. H.; Dubree, N. J. P.; Jones, R. J.; Kent, K. M.; Lee, T. T.; Louie, M. S.; Postich, M. J.; Prise, E. J.; Rohloff, J. C.; Yu, R. H.; Tetrahedron Lett. 1998, 39, 1853. (j) Lohray, B. B.; Reddy, A. S.; Bhushan, V. Tetrahedron: Asymmetry 1996, 7, 2411. (k) Smith III, A. B.; Chen, S. S.-Y.; Nelson, F. C.; Reichert, J. M.; Salvatore, B. A. J. Am. Chem. Soc. 1995, 117, 12013. (1) Parida, S.; Dordick, J. S. J. Am. Chem. Soc. 1991, 113, 2253. (m) Roush, W. R.; Lin, X.; Straub, J. A. J. Org. Chem. 1991, 56, 1649. (n) Nelson, W. L.; Wennerstrom, J. E.; Sankar, S. R. J. Org. Chem. 1977, 42, 1006.

2. (a) Qin, T.; Li, J.-P.; Xie, M.-S.; Qu, G.-R.; Guo, H.-M. J. Org. Chem. 2018, 83, 15512. (b) Branco, L. C.; Serbanovic, A.; da Ponte, M. N.; Afonso, C. A. M. ACS Catal. 2011, 1, 1408. (c) Smaltz, D. J.; Myers, A. G. J. Org. Chem. 2011, 76, 8554. (d) Zaitsev, A. B.; Adolfsson, H. Synthesis 2006, 1725. (e) Choudary, B. M.; Chowdari, N. S.; Jyothi, K.; Kantam, M. L. J. Am. Chem. Soc. 2002, 124, 5441. (f) Jonsson, S. Y.; Adolfsson, H.; Ba1ckvall, J-E. Org. Lett. 2001, 22, 3463. (g) Eames, J.; Mitchell, H. J.; Nelson, A.; Brien, P. O.; Warren, S.; Wyatt, P. J. Chem. Soc., Perkin Trans. 1, 1999, 1095. (h) Kolb, H. C.; VanNieuwenhze, M. S.; Sharpless, K. B. Chem. Rev. 1994, 94, 2483. (i) Jacobsen, E. N.; Marko, I.; Mungall, W. S.; Schrçder, G.; Sharpless, K. B. J. Am. Chem. Soc. 1988, 110, 1968. (j) VanRheenen, V.; Kelly, R. C.; Cha, D. Y. Tetrahedron Lett. 1976, 17, 1067.

3. (a) Hu, W.-X.; Li, P.-R.; Jiang, G.; Che, C.-M.; Chen, J. Adv. Synth. Catal. 2010, 352, 3190. (b) Neisius, N. M.; Plietker, B. J. Org. Chem. 2008, 73, 3218. (c) Plietker, B.; Niggemann, M. J. Org. Chem. 2005, 70, 2402. (d) Plietker, B.; Niggemann, M. Org. Lett. 2003, 5, 3353. (e) Shing, T. K. M.; Tam, E. K. W.; Tai, V. W. F.; Chung, I. H. F.; Jiang, Q.; Chem. Eur. J. 1996, 2, 50. 
4. (a) Fan, T.; Shen, H.-C.; Han Z.-Y.; Gong, L.-Z. Chin. J. Chem. 2019, 37, 226. (b) Enthaler, S. Chem. Soc. Rev. 2011, 40, 4912. (c) Wang, A.; Jiang, H. J. Org. Chem. 2010, 75, 2321.

5. (a) de Boer, J. W.; Browne, W. R.; Brinksma, J.; Alsters, P. L.; Hage, R.; Feringa, B. L. Inorg. Chem. 2007, 46, 6353. (b) de Boer, J. W.; Brinksma, J.; Browne, W. R.; Meetsma, A.; Alsters, P. L.; Hage, R.; Feringa, B. L. J. Am. Chem. Soc. 2005, 127, 7990 (c) De Vos, D. E.; de Wildeman, S.; Sels, B. F.; Grobet, P. J.; Jacobs, P. A. Angew. Chem. Int. Ed. 1999, 38, 980.

6. (a) Wei, J.; Wu, L.; Wang, H.-X.; Zhang, X.; Tse, C. W.; Zhou, C. Y.; Huang, J.-S.; Che, C.-M. Angew. Chem. Int. Ed. 2020, 59 , 16561. (b) Chow, T. W.-S.; Wong, E. L.-M.; Guo, Z.; Liu, Y.; Huang, J.-S.; Che, C.-M. J. Am. Chem. Soc. 2010, 132, 13229. (c) Aciro, C.; Davies, S. G., Kurosawa, W.; Roberts, P. M.; Russell, A. J.; Thomson, J. E.; Org. Lett. 2009, 11, 1333. (d) Oldenburg, P. D.; Jr., Que, L. Catal. Today 2006, 117, 15. (e) Oldenburg, P. D.; Shteinman, A. A.; Jr, Que L. J. Am. Chem. Soc. 2005, 127, 15672. (f) Emmanuvel, L.; Shaikh, T. M. A.; Sudalai, A. Org. Lett. 2005, 7, 5071. (h) Chen, K.; Costas, M.; Kim, J.; Tipton, A. K.; Jr., Que, L. J. Am. Chem. Soc. 2002, 124, 3026. (g) Oldenburg, P. D.; Shteinman, A. A.; Jr., Que, L. J. Am. Chem. Soc. 2005, 127 15672. (h) Klopstra, M.; Roelfes, G.; Hage, R.; Kellogg, R. M.; Feringa, B. L. Eur. J. Inorg. Chem. 2004, 846. (i) Costas, M.; Tipton, A. K.; Chen, K.; Jo, D.-H.; Jr., Que, L. J. Am. Chem. Soc. 2001, 123, 6722 .

7. (a) Pilevar, A.; Hosseini, A.; Becker, J.; Schreiner, P. R. J. Org. Chem. 2019, 84, 12377. (b) Alamillo-Ferrer, C.; Davidson S. C.; Rawling, M. J.; Theodoulou, N. H.; Campbell M.; Humphreys, P. G.; Kennedy, A. R.; Tomkinson, N. C. Org. Lett. 2015, $20,5132$. (c) Picon, S.; Rawling, M.; Campbell, M.; Tomkinson, N. C. Org. Lett. 2012, 24, 6250. (d) Griffith, J. C.; Jones, K. M.; Picon, S.; Rawling, M. J.; Kariuki, B. M.; Campbell, M.; Tomkinson, N. C. J. Am. Chem. Soc. 2010, 132, 14409.

8. (a) Giglio, B. C.; Schmidt, V. A.; Alexanian, E. J. J. Am. Chem. Soc. 2011, 133, 13320. (b) Schmidt, V. A.; Alexanian, E. J. Angew. Chem. Int. Ed. 2010, 49, 4491.
9. For recent reviews see: (a) Yoshimura, A.; Zhdankin V. V. Chem Rev. 2016, 116, 3328. (b) Parra, A. Chem. Rev. 2019, 119, 12033. For articles see: (c) Bekkaye, M.; Su, Y.; Masson, G. Eur. J. Org. Chem. 2013, 2013, 3978. (d) Zhong, W.; Liu, S.; Yang, J.; Meng, X.; Li, Z. Org. Lett. 2012, 14, 3336. (e) Zhong, W.; Yang, J.; Meng, X.; Li, Z. J. Org. Chem. 2011, 76, 9997. (f) Celik, M.; Alp, C.; Coşkun, B.; Gültekin, M. S.; Balci, M. Tetrahedron Lett. 2006, 47, 3659. (g) Emmanuvel, L.; Shaikh, T. M. A.; Sudalai, A. Org. Lett. 2005, 7, 5071

10. (a) Albrecht, L.; Jiang, H.; Dickmeiss, G. Gschwend, B.; Hansen, S. G.; Jørgensen K. A. J. Am. Chem. Soc. 2010, 132, 9188. (b) Bhunnoo, R. A.; Hu, Y.; Lainé, D. I.; Brown, R. C. D. Angew. Chem. Int. Ed. 2002, 41, 3479.

11. (a) Hu, D.; Zong, X.-C.; Xue, F.; Li, C.; Hu, B.-C.; Wu, M.-C. Chem. Commun. 2020, 56, 2799. (b) Wu, S.; Zhou, Y.; Li, Z. Chem. Commun. 2019, 55, 883. (c) de Gonzalo, G. Molecules, 2018, 23, 1585. (d) Lewis, S. E. Chem. Commun. 2014, 50, 2821. (e) Boyd, D. R.; Sharma, N. D.; Bowers, N. I.; Brannigan, I. N.; Groocock, M. R.; Malone, J. F.; McConville, G.; Allen, C. C. Adv. Synth. Catal. 2005, 347, 1081

12. Fujita, M.; Wakita, M.; Sugimura, T. Chem. Commun. 2011, 47, 3983.

13. (a) Tian, B.; Chen, P.; Leng, X.; Liu, G. Nat. Catal. 2021, 4, 172. (b) Thirsten H. W.; Muñiz, K. Georg Thieme Verlag Stuttgart. New York, 2016, doi.org/10.1055/s-0035-1561313. (c) AlamilloFerrer, C.; Davidson, S. C.; Rawling, M. J.; Theodoulou, N. H.; Campbell, M.; Humphreys, P. G.; Kennedy, A. R.; Tomkinson, N. C. O. Org. Lett. 2015, 17, 5132. And references cited in 9.

14. Zhang, Y.; Sigman, M. S. J. Am. Chem. Soc. 2007, 129, 3076.

15. CCDC 1946629 contains the supplementary crystallographic data for this paper. These data can be obtained free of charge from The Cambridge Crystallographic Data Centre via https://www.ccdc.cam.ac.uk/daa_request/cif.

16. HPLC separation for $\mathbf{2 z}$ was not achieved, see SI for further details. 


\title{
Supplementary Information
}

\section{Catalytic Enantioselective syn-Hydroxy-Oxyacylation of Electron Deficient Alkenes}

\author{
Chandra Bhan Pandey, ${ }^{\dagger}$ Vikram Singh, ${ }^{\dagger}$ Susanta Ghanta, ${ }^{\star}$ and Bhoopendra Tiwari ${ }^{\dagger *}$ \\ 'Division of Molecular Synthesis \& Drug Discovery, Centre of Biomedical Research, SGPGIMS-Campus, Raebareli \\ Road, Lucknow, 226014, India. \\ Department of Chemistry, National Institute of Technology, Agartala, 799046, Tripura, India.
}

\section{Table of Contents}

I General information $\quad$ S2

II General procedures for the synthesis of Chalcones 1

III General Procedure for synthesis of Hydroxy-oxyacylation products $2 \quad$ S2

IV References S3

V Stereochemistry determination of $\mathbf{2 d}$ via X-ray crystallographic analysis $\quad$ S3

VI Characterization data of Hydroxy-oxyacylation Products, 2

VII $\quad{ }^{1} \mathrm{H}$ and $\left.{ }^{13} \mathrm{C}_{\{1}{ }^{1} \mathrm{H}\right\}$ spectra of Hydroxy-oxyacylation products, $2 \quad \mathrm{~S} 12$

VIII HPLC spectra of Hydroxy-oxyacylation products, 2 


\section{General Information}

Commercially available aldehydes purchased from Alfa-Aesar or Sigma-Aldrich were used without further purification. The chalcones were synthesized from commercially available aryl aldehydes and aryl acetophenones following the literature procedure. ${ }^{1}$ The chiral catalysts were synthesized using the reported literature procedure. ${ }^{2}$ Solvents were dried and distilled following the standard methods. TLC was carried out on pre-coated plates (Merck silica gel 60, f254), and the spots were visualized with UV light and by charring the plates dipped in phosphomolybdic acid (PMA) solution. Column chromatography was accomplished using silica gel (230-400 mesh) with distilled solvents. Proton nuclear magnetic resonance $\left({ }^{1} \mathrm{H}\right)$ and proton decoupled carbon nuclear magnetic resonance $\left({ }^{13} \mathrm{C}\left\{{ }^{1} \mathrm{H}\right\}\right)$ for compounds were recorded at $400 \mathrm{MHz}$ and $800 \mathrm{MHz}$ instruments using $\mathrm{CDCl}_{3}$ as the solvent. Chemical shifts were recorded in parts per million (ppm, $\delta)$ relative to tetramethylsilane $(\delta 0.00)$ or chloroform $(\delta 7.26$, singlet $) .{ }^{1} \mathrm{H}$ NMR splitting patterns are designated as singlet (s), doublet (d), triplet (t), quartet (q), dd (doublet of doublets), m (multiplets), and etc. All first-order splitting patterns were assigned on the basis of the appearance of the peaks. High-resolution mass spectral analysis (HRMS) was performed on "QTOF Premier mass spectrometer". HPLC analysis was performed on "Agilent Technologies 1260 Infinity" using chiral stationary phase.

\section{General procedures for the synthesis of Chalcones 1}

Chalcones 1, were synthesized by an Aldol-condensation reaction, following a literature procedure. ${ }^{[1]}$

To a stirred solution of substituted acetophenone $(20 \mathrm{mmol})$ in ethanol $(50 \mathrm{~mL})$ at $0^{\circ} \mathrm{C}$, was added dropwise a solution of sodium hydroxide $(26 \mathrm{mmol})$ in water $(15 \mathrm{~mL})$ followed by slow addition of substituted benzaldehyde $(20 \mathrm{mmol})$. The resulting mixture was slowly warmed to room temperature and stirred for $6 \mathrm{~h}$, then filtered and washed with ethanol to get the pure product $\mathbf{1}$.

\section{General Procedure for synthesis of Hydroxy-oxyacylation products 2}

To a $25 \mathrm{ml}$ round bottom flask containing magnetic bar was added catalyst $\mathbf{E}(0.02 \mathrm{mmol})$ and $\mathrm{m}$ CPBA (0.12 mmol, 1.20 equiv.) followed by acetic acid (1.0 mL) and trifluoroacetic acid (1.0 ml) at room temperature. Chalcone $\mathbf{1}(0.1 \mathrm{mmol})$ was then added in one portion followed by $\mathrm{BF}_{3} . \mathrm{OEt}_{2}$ $(0.05 \mathrm{mmol}, 50 \mathrm{~mol} \%)$ and the resulting mixture was allowed to stir at $\mathrm{rt}$ for $12 \mathrm{~h}$. After completion, the reaction mixture was quenched with a saturated aqueous solution of $\mathrm{NaHCO}_{3}$ and extracted 
with $\mathrm{CH}_{2} \mathrm{Cl}_{2}(3 \times 5 \mathrm{~mL})$. The combined organic layers were dried over $\mathrm{Na}_{2} \mathrm{SO}_{4}$, and concentrated in vacuo to provide the crude product. The crude mixture was then purified by column chromatography to afford the pure product.

\section{References}

1. Farid, U.; Malmedy, F.; Claveau, R.; Albers, L.; Wirth, T. Angew. Chem., Int. Ed. 2013, 52, 7018.

2. For a catalyst preparation see: (a) Pandey, C. B.; Azaz, T.; Verma, R. S.; Mishra, M.; Jat, J. L.; Tiwari, B. J. Org. Chem. 2020, 85, 10175. (b) Muñiz, K.; Barreiro, L.; Romero, M.; Martínez, C. J. Am. Chem. Soc. 2017, 139, 4354. (c) Haubenreisser, S.; Wöste, T. H.; Martínez, C.; Ishihara, K.; Muñiz, K. Angew. Chem, Int. Ed. 2016, 55, 413. (d) Banik, S. M.; Medley, J. W.; Jacobsen, E. N. J. Am. Chem. Soc. 2016, 138, 5000. (e) Banik, S. M.; Medley, J. W.; Jacobsen, E. N. Catalytic, Science 2016, 353, 51. (f) Woerly, E. M.; Banik, S. M.; Jacobsen, E. N. J. Am. Chem. Soc. 2016, 138, 13858. (g) Yoshida, Y.; Magara, A.; Mino, T.; Sakamoto, M. Tetrahedron Lett. 2016, 57, 5103. (h) Uyanik, M.; Yasui, T.; Ishihara, K. Tetrahedron 2010, 66, 5841. (i) Uyanik, M.; Yasui, T.; Ishihara, K. Angew. Chem., Int. Ed. 2010, 49, 2175.

\section{Stereochemistry determination of $2 \mathrm{~d}$ via $\mathrm{X}$-ray crystallographic analysis}

Absolute configurations of the products were assigned based on the crystal X-ray structures of $\mathbf{2 d}$. A colorless flaky crystal of $\mathbf{2 d}$ was obtained by vaporization of a methanol solution of compound 2d $(96 \%$ ee). The absolute configuration of $\mathbf{2 d}$ was determined by X-ray to be $(1 S, 2 R)$. CCDC 1946629 contains the supplementary X-ray crystallographic data. These data can be obtained free of charge from The Cambridge Crystallographic Data Centre via www.ccdc.cam.ac.uk/data request/cif.<smiles>CC(=O)O[C@H](c1ccccc1)[C@H](O)C(=O)c1ccc(Br)cc1</smiles>

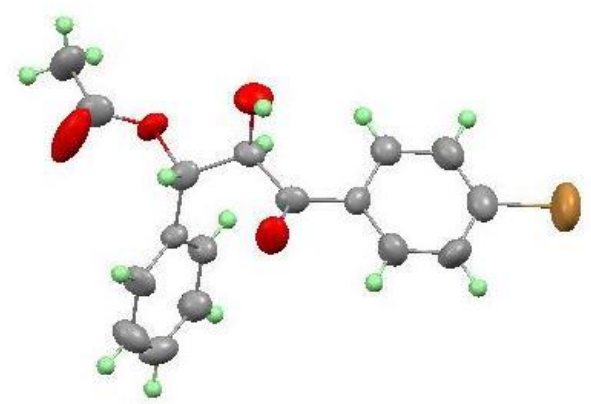




\section{Characterization data of Hydroxy-oxyacylation Products 2}

(1S,2R)-2-Hydroxy-3-oxo-1,3-diphenylpropyl acetate (2a): Colorless gummy liquid, 96\% yield

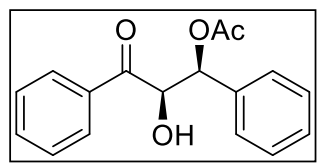

$(27.3 \mathrm{mg}) ;[\alpha]_{\mathrm{D}}{ }^{20}=-27.4\left(c=0.1 \mathrm{CHCl}_{3}\right) ;{ }^{1} \mathrm{H} \mathrm{NMR}\left(400 \mathrm{MHz}, \mathrm{CDCl}_{3}\right) \delta$ 7.93-7.87 (m, 2H), $7.64(\mathrm{t}, J=8.0 \mathrm{~Hz}, 1 \mathrm{H}), 7.51(\mathrm{t}, J=8.0 \mathrm{~Hz}, 1 \mathrm{H}), 7.42-$ $7.28(\mathrm{~m}, 5 \mathrm{H}), 6.09(\mathrm{~d}, J=4 \mathrm{~Hz}, 1 \mathrm{H}), 5.35(\mathrm{dd}, J=8 \mathrm{~Hz}, J=2.9 \mathrm{~Hz}, 1 \mathrm{H})$; $3.84(\mathrm{~d}, J=8.0 \mathrm{~Hz}, 1 \mathrm{H}) ; 2.00(\mathrm{~s}, 3 \mathrm{H}) ;{ }^{13} \mathrm{C}\left\{{ }^{1} \mathrm{H}\right\} \mathrm{NMR}\left(100 \mathrm{MHz}, \mathrm{CDCl}_{3}\right) \delta 198.7,169.4,136.5$, 134.1, 134.0, 128.8, 128.4, 128.3, 126.9, 75.5, 75.1, 20.6; HRMS (ESI) $\mathrm{m} / \mathrm{z}$ calcd. for $\mathrm{C}_{17} \mathrm{H}_{16} \mathrm{O}_{4} \mathrm{Na}$ $[\mathrm{M}+\mathrm{Na}]^{+}$: 307.0941, found: 307.0939; HPLC analysis: 95\% ee (Chiralcel OD-H, 02:98 $\left.{ }^{\mathrm{i}} \mathrm{PrOH} / \mathrm{Hexane}, 1 \mathrm{~mL} / \mathrm{min}\right), \mathrm{R}_{\mathrm{t}}($ minor $)=46.5 \mathrm{~min}, \mathrm{R}_{\mathrm{t}}$ (major) $=33.5 \mathrm{~min}$.

$(1 S, 2 R)$-2-Hydroxy-3-oxo-1-phenyl-3-(p-tolyl)propyl acetate (2b): Colorless gummy liquid,

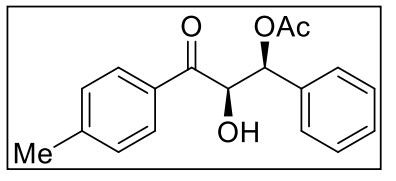
$73 \%$ yield $(21.7 \mathrm{mg}) ;[\alpha]_{\mathrm{D}}{ }^{20}=+12.6\left(c=0.1 \mathrm{CHCl}_{3}\right) ;{ }^{1} \mathrm{H} \mathrm{NMR} \quad(400$ $\left.\mathrm{MHz}, \mathrm{CDCl}_{3}\right) \delta 7.82(\mathrm{~d}, J=8.0 \mathrm{~Hz}, 2 \mathrm{H}), 7.43-7.28(\mathrm{~m}, 7 \mathrm{H}), 6.09(\mathrm{~d}, J$ $=2.6,1 \mathrm{H}), 5.31(\mathrm{dd}, J=8 \mathrm{~Hz}, 2.6 \mathrm{~Hz}, 1 \mathrm{H}), 3.88(\mathrm{~d}, J=8.0 \mathrm{~Hz}, 1 \mathrm{H}), 2.44$ (s, 3H), 2.01(s, 3H), ${ }^{13} \mathrm{C}\left\{{ }^{1} \mathrm{H}\right\}$ NMR (100 MHz, $\left.\mathrm{CDCl}_{3}\right) \delta 198.1,169.4,145.2,136.6,131.4,129.6$, 128.5, 128.3, 126.9, 75.7, 75.0, 21.7, 20.6; HRMS (ESI) m/z calcd for $\mathrm{C}_{18} \mathrm{H}_{18} \mathrm{O}_{4} \mathrm{Na}[\mathrm{M}+\mathrm{Na}]^{+}$: 321.1097, found: 321.1098; HPLC analysis: 98\% ee (Chiralcel OD-H, 02:98 ${ }^{\mathrm{i}} \mathrm{PrOH} / \mathrm{Hexane}$, $1 \mathrm{~mL} / \mathrm{min}), \mathrm{R}_{\mathrm{t}}($ minor $)=38.3 \mathrm{~min}, \mathrm{R}_{\mathrm{t}}($ major $)=25.0 \mathrm{~min}$.

(1S,2R)-2-Hydroxy-3-(4-methoxyphenyl)-3-oxo-1-phenylpropyl acetate (2c): Colorless

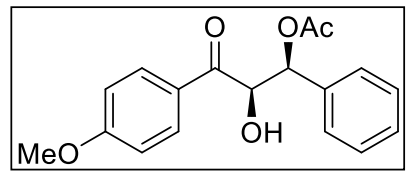
gummy liquid, $93 \%$ yield $(29.2 \mathrm{mg}) ;[\alpha]_{\mathrm{D}}{ }^{20}=+4.4\left(c=0.1 \mathrm{CHCl}_{3}\right) ;{ }^{1} \mathrm{H}$ NMR $\left(400 \mathrm{MHz}, \mathrm{CDCl}_{3}\right) \delta 7.80(\mathrm{dd}, J=8.0 \mathrm{~Hz}, 4.0 \mathrm{~Hz}, 2 \mathrm{H}), 7.33-$ $7.28(\mathrm{~m}, 2 \mathrm{H}), 7.27-7.19(\mathrm{~m}, 3 \mathrm{H}), 6.90-6.85(\mathrm{~m}, 2 \mathrm{H}), 6.00(\mathrm{~d}, J=4.0$ $\mathrm{Hz}, 1 \mathrm{H}), 5.21-5.16(\mathrm{~m}, 1 \mathrm{H}), 3.82(\mathrm{~d}, J=8.0 \mathrm{~Hz}, 1 \mathrm{H}), 3.78(\mathrm{~s}, 3 \mathrm{H}), 1.92(\mathrm{~s}, 3 \mathrm{H}) ;{ }^{13} \mathrm{C}\left\{{ }^{1} \mathrm{H}\right\} \mathrm{NMR}$ $\left(100 \mathrm{MHz}, \mathrm{CDCl}_{3}\right) \delta 196.6,169.4,164.2,136.7,130.8,128.3,126.8,126.7,114.1,75.8,74.7$, 55.4, 20.6; HRMS (ESI) m/z calcd for $\mathrm{C}_{18} \mathrm{H}_{18} \mathrm{O}_{5} \mathrm{Na}$ [M+Na] ${ }^{+}: 337.1046$, found: 337.1046; HPLC analysis: $89 \%$ ee (Chiralcel IC, $\left.10: 90{ }^{i} \mathrm{PrOH} / \mathrm{Hexane}, 1 \mathrm{~mL} / \mathrm{min}\right), \mathrm{R}_{\mathrm{t}}($ minor $)=54.6 \mathrm{~min}$, $\mathrm{R}_{\mathrm{t}}($ major $)=44.0 \mathrm{~min}$.

(1S,2R)-3-(4-Bromophenyl)-2-hydroxy-3-oxo-1-phenylpropyl acetate (2d): Colorless gummy

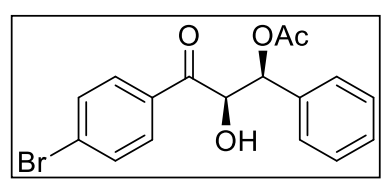
liquid, $76 \%$ yield $(27.5 \mathrm{mg}) ;[\alpha]_{\mathrm{D}}{ }^{20}=+25.8\left(c=0.1 \mathrm{CHCl}_{3}\right) ;{ }^{1} \mathrm{H} \mathrm{NMR}$ $\left(400 \mathrm{MHz}, \mathrm{CDCl}_{3}\right) \delta 7.75(\mathrm{~d}, J=8.0 \mathrm{~Hz}, 2 \mathrm{H}), 7.64(\mathrm{~d}, J=12.0 \mathrm{~Hz}, 2 \mathrm{H})$, 7.40-7.27 (m, 5H), $6.05(\mathrm{~d}, J=4.0 \mathrm{~Hz}, 1 \mathrm{H}), 5.27(\mathrm{dd}, J=8.0 \mathrm{~Hz}, 4.0 \mathrm{~Hz}$, $1 \mathrm{H}), 3.75(\mathrm{~d}, J=8.0 \mathrm{~Hz}, 1 \mathrm{H}), 2.02(\mathrm{~s}, 3 \mathrm{H}) ;{ }^{13} \mathrm{C}\left\{{ }^{1} \mathrm{H}\right\} \mathrm{NMR}\left(100 \mathrm{MHz}, \mathrm{CDCl}_{3}\right) \delta 197.8,169.5$, 
136.2, 132.9, 132.2, 129.8, 129.4, 128.5, 128.4, 126.9, 75.5, 75.2, 20.7; HRMS (ESI) calcd for OCl $\mathrm{C}_{17} \mathrm{H}_{15} \mathrm{BrO}_{4} \mathrm{Na}[\mathrm{M}+\mathrm{Na}]^{+}:$385.0046, found: 385.0039; HPLC analysis: 96\% ee (Chiralcel OD-H, 02:98 $\left.{ }^{i} \mathrm{PrOH} / \mathrm{Hexane}, 1 \mathrm{~mL} / \mathrm{min}\right), \mathrm{R}_{\mathrm{t}}$ (minor) $=65.1 \mathrm{~min}, \mathrm{R}_{\mathrm{t}}($ major $)=37.0 \mathrm{~min}$.

(1S,2R)-3-(4-Chlorophenyl)-2-hydroxy-3-oxo-1-phenylpropyl acetate (2e): Colorless gummy (1) liquid, $64 \%$ yield $(20.4 \mathrm{mg}) ;[\alpha]_{\mathrm{D}}{ }^{20}=+68.8\left(c=0.1 \mathrm{CHCl}_{3}\right) ;{ }^{1} \mathrm{H} \mathrm{NMR}$ $\left(400 \mathrm{MHz}, \mathrm{CDCl}_{3}\right) \delta 7.82(\mathrm{~d}, J=8.0 \mathrm{~Hz}, 2 \mathrm{H}), 7.47(\mathrm{~d}, J=8.0 \mathrm{~Hz}, 2 \mathrm{H})$, $7.38-7.28(\mathrm{~m}, 5 \mathrm{H}), 6.05(\mathrm{~d}, J=2.9 \mathrm{~Hz}, 1 \mathrm{H}), 5.27(\mathrm{dd}, J=8.0 \mathrm{~Hz}, 4.0$ $\mathrm{Hz}, 1 \mathrm{H}), 3.73(\mathrm{~d}, J=8.0 \mathrm{~Hz}, 1 \mathrm{H}), 2.02(\mathrm{~s}, 3 \mathrm{H}) ;{ }^{13} \mathrm{C}\left\{{ }^{1} \mathrm{H}\right\} \mathrm{NMR}\left(100 \mathrm{MHz}, \mathrm{CDCl}_{3}\right) \delta$ 197.6, 169.5, 140.6, 136.2, 132.5, 129.8, 129.2, 128.5, 128.4, 126.9, 75.5, 75.2, 20.7; HRMS (ESI) m/z calcd for $\mathrm{C}_{17} \mathrm{H}_{15} \mathrm{ClO}_{4} \mathrm{Na}$ [M+Na] ${ }^{+}: 341.0551$, found: 341.0546; HPLC analysis: $96 \%$ ee (Chiralcel OD$\left.\mathrm{H}, 02: 98{ }^{i} \mathrm{PrOH} / \mathrm{Hexane}, 1 \mathrm{~mL} / \mathrm{min}\right), \mathrm{R}_{\mathrm{t}}($ minor $)=61.8 \mathrm{~min}, \mathrm{R}_{\mathrm{t}}($ major $)=34.1 \mathrm{~min}$.

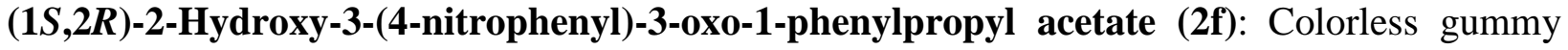

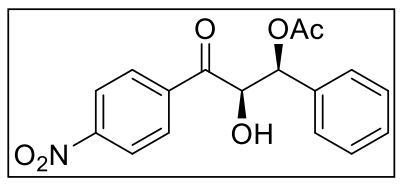
liquid, $21 \%$ yield $(7 \mathrm{mg}) ;[\alpha]_{\mathrm{D}}{ }^{20}=+19.8\left(c=0.1 \mathrm{CHCl}_{3}\right) ;{ }^{1} \mathrm{H} \mathrm{NMR}$ $\left(400 \mathrm{MHz}, \mathrm{CDCl}_{3}\right) \delta 8.27(\mathrm{~d}, J=8.0 \mathrm{~Hz}, 2 \mathrm{H}), 7.96(\mathrm{~d}, J=8.0 \mathrm{~Hz}, 2 \mathrm{H})$, $7.30-7.22(\mathrm{~m}, 5 \mathrm{H}), 5.98(\mathrm{~d}, J=3.3 \mathrm{~Hz}, 1 \mathrm{H}), 5.26(\mathrm{~s}, 1 \mathrm{H}), 3.56(\mathrm{~d}, J=$ $8.0 \mathrm{~Hz}, 1 \mathrm{H}), 1.97(\mathrm{~s}, 3 \mathrm{H}) ;{ }^{13} \mathrm{C}\left\{{ }^{1} \mathrm{H}\right\} \mathrm{NMR}\left(100 \mathrm{MHz}, \mathrm{CDCl}_{3}\right) \delta 197.9,169.6,150.6,139.1,135.8$, 129.5, 128.8, 128.6, 126.9, 123.9, 75.9, 75.3, 29.6; HRMS (ESI) m/z calcd. for $\mathrm{C}_{17} \mathrm{H}_{15} \mathrm{NO}_{6} \mathrm{Na}$ $[\mathrm{M}+\mathrm{Na}]^{+}:$352.0792, found: 352.0793; HPLC analysis: 92\% ee (Chiralcel OD-H, 02:98 ${ }^{i} \mathrm{PrOH} /$ Hexane, $\left.1 \mathrm{~mL} / \mathrm{min}\right), \mathrm{R}_{\mathrm{t}}$ (minor) $=58.6 \mathrm{~min}, \mathrm{R}_{\mathrm{t}}$ (major) $=32.9 \mathrm{~min}$.

$(1 S, 2 R)$-2-Hydroxy-3-oxo-3-phenyl-1-(p-tolyl)propyl acetate (2g): Colorless gummy liquid,

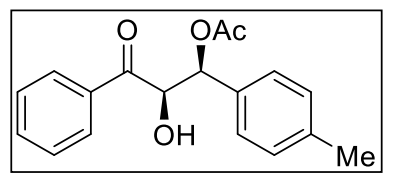
$68 \%$ yield $(20.3 \mathrm{mg}) ;[\alpha]_{\mathrm{D}}{ }^{20}=+16.2\left(c=0.1 \mathrm{CHCl}_{3}\right) ;{ }^{1} \mathrm{H}$ NMR $(400$ $\left.\mathrm{MHz}, \mathrm{CDCl}_{3}\right) \delta 7.81(\mathrm{~d}, J=8.0 \mathrm{~Hz}, 2 \mathrm{H}), 7.55(\mathrm{t}, J=8.0 \mathrm{~Hz}, 3 \mathrm{H}), 7.43$ $(\mathrm{t}, J=8.0,2 \mathrm{H}), 7.19(\mathrm{t}, J=8.0,2 \mathrm{H}), 7.07(\mathrm{~d}, J=8.0,2 \mathrm{H}), 5.97(\mathrm{~d}, J=$ 4.0, 1H), $5.24(\mathrm{dd}, J=8.0 \mathrm{~Hz}, 4.0 \mathrm{~Hz}, 1 \mathrm{H}), 3.72(\mathrm{~d}, J=8.0 \mathrm{~Hz}, 1 \mathrm{H}), 2.25(\mathrm{~s}, 3 \mathrm{H}), 1.90(\mathrm{~s}, 3 \mathrm{H})$; ${ }^{13} \mathrm{C}\left\{{ }^{1} \mathrm{H}\right\} \mathrm{NMR}\left(100 \mathrm{MHz}, \mathrm{CDCl}_{3}\right) \delta 198.8,169.4,138.2,134.2,134.0,133.5,129.0,128.8,128.4$, 126.8, 75.5, 75.1, 21.1, 20.6; HRMS (ESI) calcd for $\mathrm{C}_{18} \mathrm{H}_{18} \mathrm{O}_{4} \mathrm{Na}[\mathrm{M}+\mathrm{Na}]^{+}: 321.1097$, found: 321.1096; HPLC analysis: 83\% ee (Chiralcel OD-H, 02:98 $\left.{ }^{i} \mathrm{PrOH} / \mathrm{Hexane}, 1 \mathrm{~mL} / \mathrm{min}\right), \mathrm{R}_{\mathrm{t}}(\mathrm{minor})$ $=29.8 \mathrm{~min}, \mathrm{R}_{\mathrm{t}}$ (major) $=43.8 \mathrm{~min}$.

(1S,2R)-1-(4-Chlorophenyl)-2-hydroxy-3-oxo-3-phenylpropyl acetate (2h): Colorless gummy liquid, $60 \%$ yield $(19 \mathrm{mg}) ;[\alpha]_{\mathrm{D}}{ }^{20}=+61.6\left(c=0.1 \mathrm{CHCl}_{3}\right) ;{ }^{1} \mathrm{H} \mathrm{NMR}\left(400 \mathrm{MHz}, \mathrm{CDCl}_{3}\right) \delta 7.88$ 
$(\mathrm{d}, J=8.0 \mathrm{~Hz}, 2 \mathrm{H}), 7.65(\mathrm{t}, J=8.0 \mathrm{~Hz}, 1 \mathrm{H}), 7.52(\mathrm{t}, J=8.0 \mathrm{~Hz}, 2 \mathrm{H}), 7.31(\mathrm{~s}, 4 \mathrm{H}), 6.03(\mathrm{~d}, J=$ $2.6 \mathrm{~Hz}, 1 \mathrm{H}), 5.29(\mathrm{dd}, J=8.0 \mathrm{~Hz}, 4.0 \mathrm{~Hz}, 1 \mathrm{H}), 3.84(\mathrm{~d}, J=8.0 \mathrm{~Hz}, 1 \mathrm{H}), 1.98(\mathrm{~s}, 3 \mathrm{H}) ;{ }^{13} \mathrm{C}\left\{{ }^{1} \mathrm{H}\right\}$ NMR $\left(100 \mathrm{MHz}, \mathrm{CDCl}_{3}\right) \delta 198.4,169.3,135.0,134.3,134.2,134.0,128.9,128.5,128.4,75.0$, 74.9, 20.6; HRMS (ESI) $\mathrm{m} / \mathrm{z}$ calcd for $\mathrm{C}_{17} \mathrm{H}_{15} \mathrm{ClO}_{4} \mathrm{Na}[\mathrm{M}+\mathrm{Na}]^{+}$: 341.0551, found: 341.0546; HPLC analysis: 94\% ee (Chiralcel OD-H, 02:98 $\left.{ }^{\mathrm{P}} \mathrm{PrOH} / \mathrm{Hexane}, 1 \mathrm{~mL} / \mathrm{min}\right), \mathrm{R}_{\mathrm{t}}(\mathrm{minor})=$ $33.8 \mathrm{~min}, \mathrm{R}_{\mathrm{t}}($ major $)=36.5 \mathrm{~min}$.

(1S,2R)-1-(2-Bromophenyl)-2-hydroxy-3-oxo-3-phenylpropyl acetate (2i): Colorless gummy

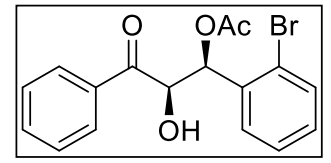
liquid, $62 \%$ yield $(22.6 \mathrm{mg}) ;[\alpha]_{\mathrm{D}}{ }^{20}=+14.2\left(c=0.1 \mathrm{CHCl}_{3}\right) ;{ }^{1} \mathrm{H}$ NMR $(400$ $\left.\mathrm{MHz}, \mathrm{CDCl}_{3}\right) \delta 7.77-7.73(\mathrm{~m}, 2 \mathrm{H}), 7.67-7.63(\mathrm{~m}, 2 \mathrm{H}), 7.38-7.30(\mathrm{~m}, 5 \mathrm{H})$, $6.05(\mathrm{~d}, J=4.0 \mathrm{~Hz}, 1 \mathrm{H}), \quad 5.26(\mathrm{~d}, J=8.0 \mathrm{~Hz}, 1 \mathrm{H}), 3.75(\mathrm{~s}, 1 \mathrm{H}), 2.02(\mathrm{~s}, 3 \mathrm{H}) ;{ }^{13} \mathrm{C}\left\{{ }^{1} \mathrm{H}\right\}$ NMR $(100$ $\left.\mathrm{MHz}, \mathrm{CDCl}_{3}\right) \delta 197.9,169.5,136.3,133.0,132.2,129.9,129.5,128.6,128.5,127.0,75.6,75.2$, 20.7; HRMS (ESI) $\mathrm{m} / \mathrm{z}$ calcd. for $\mathrm{C}_{17} \mathrm{H}_{15} \mathrm{BrO}_{4} \mathrm{Na}$ [M+Na] ${ }^{+}$: 387.0025, found: 387.0021; HPLC analysis: 96\% ee (Chiralcel OD-H, 02:98 $\left.{ }^{i} \mathrm{PrOH} / \mathrm{Hexane}, 1 \mathrm{~mL} / \mathrm{min}\right), \mathrm{R}_{\mathrm{t}}(\mathrm{minor})=64.4 \mathrm{~min}$, $\mathrm{R}_{\mathrm{t}}($ major $)=36.8 \mathrm{~min}$.

(1S,2R)-1-(4-Bromophenyl)-2-hydroxy-3-oxo-3-phenylpropyl acetate (2j): Colorless gummy OH liquid, $65 \%$ yield $(23.7 \mathrm{mg}) ;[\alpha]_{\mathrm{D}}{ }^{20}=+36.4\left(c=0.1 \mathrm{CHCl}_{3}\right) ;{ }^{1} \mathrm{H} \mathrm{NMR}$ $\left(400 \mathrm{MHz}, \mathrm{CDCl}_{3}\right) \delta 7.79(\mathrm{~d}, J=8.0 \mathrm{~Hz}, 2 \mathrm{H}), 7.56(\mathrm{t}, J=8.0 \mathrm{~Hz}, 1 \mathrm{H})$, 7.35-7.46 (m, 4H), $7.17(\mathrm{~d}, J=8.0 \mathrm{~Hz}, 2 \mathrm{H}), 5.93(\mathrm{~d}, J=4.0 \mathrm{~Hz}, 1 \mathrm{H}), 5.20$ $(\mathrm{d}, J=4.0 \mathrm{~Hz}, 1 \mathrm{H}), 3.78(\mathrm{~d}, J=8.0 \mathrm{~Hz}, 1 \mathrm{H}), 1.89(\mathrm{~s}, 3 \mathrm{H}) ;{ }^{13} \mathrm{C}\left\{{ }^{1} \mathrm{H}\right\} \mathrm{NMR}\left(100 \mathrm{MHz}, \mathrm{CDCl}_{3}\right) \delta$ 198.3, 169.2, 135.5, 134.2, 134.0, 131.4, 128.9, 128.7, 128.3, 122.5 , 75.0, 74.8, 20.5; HRMS (ESI) calcd for $\mathrm{C}_{17} \mathrm{H}_{15} \mathrm{BrO}_{4} \mathrm{Na}[\mathrm{M}+\mathrm{Na}]^{+}$: 387.0025, found: 387.0026; HPLC analysis: 93\% ee (Chiralcel OD-H, 02:98 $\left.{ }^{i} \mathrm{PrOH} / \mathrm{Hexane}, 1 \mathrm{~mL} / \mathrm{min}\right), \mathrm{R}_{\mathrm{t}}($ minor $)=35.6 \mathrm{~min}, \mathrm{R}_{\mathrm{t}}($ major $)=40.9 \mathrm{~min}$. (1S,2R)-1-(4-Fluorophenyl)-2-hydroxy-3-oxo-3-phenylpropyl acetate (2k): Colorless gummy

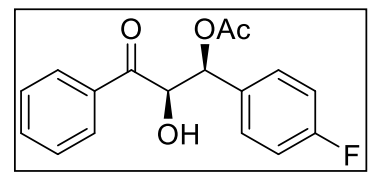
liquid, $72 \%$ yield $(21.7 \mathrm{mg}) ;[\alpha]_{\mathrm{D}}{ }^{20}=+29.8\left(c=0.1 \mathrm{CHCl}_{3}\right) ;{ }^{1} \mathrm{H} \mathrm{NMR}$ $\left(400 \mathrm{MHz}, \mathrm{CDCl}_{3}\right) \delta 7.81(\mathrm{~d}, J=8.0 \mathrm{~Hz}, 2 \mathrm{H}), 7.57(\mathrm{t}, J=8.0 \mathrm{~Hz}, 1 \mathrm{H})$, $7.45(\mathrm{t}, J=8.0 \mathrm{~Hz}, 2 \mathrm{H}), 7.33-7.27(\mathrm{~m}, 2 \mathrm{H}), 6.95(\mathrm{t}, J=8.0 \mathrm{~Hz}, 2 \mathrm{H}), 5.97$ $(\mathrm{d}, J=2.3 \mathrm{~Hz}, 1 \mathrm{H}), 5.22(\mathrm{~d}, J=4.0 \mathrm{~Hz}, 1 \mathrm{H}), 3.76(\mathrm{~d}, J=8.0 \mathrm{~Hz}, 1 \mathrm{H}), 1.91(\mathrm{~s}, 3 \mathrm{H}) ;{ }^{13} \mathrm{C}\left\{{ }^{1} \mathrm{H}\right\} \mathrm{NMR}$ $\left(100 \mathrm{MHz}, \mathrm{CDCl}_{3}\right) \delta 198.6,169.4,162.7\left(\mathrm{~d}, \mathrm{~J}_{\mathrm{C}-\mathrm{F}}=245 \mathrm{~Hz}\right) 134.2,134.1,132.4,132.3,129.0$, 128.9, 128.8, 128.4, 115.5, 115.2, 75.0, 20.6; HRMS (ESI) m/z calcd. for $\mathrm{C}_{17} \mathrm{H}_{15} \mathrm{FO}_{4} \mathrm{Na}[\mathrm{M}+\mathrm{Na}]^{+}$: 325.0847, found: 325.0839; HPLC analysis: 92\% ee (Chiralcel OD-H, 02:98 ${ }^{i} \mathrm{PrOH} / \mathrm{Hexane}, 1$ $\mathrm{mL} / \mathrm{min}), \mathrm{R}_{\mathrm{t}}($ minor $)=29.1 \mathrm{~min}, \mathrm{R}_{\mathrm{t}}($ major $)=22.8 \mathrm{~min}$. 
(1S,2R)-2-Hydroxy-3-oxo-1,3-di-p-tolylpropyl acetate (2l): Colorless gummy liquid, 60\%

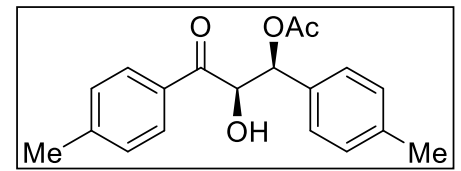
yield $(18.7 \mathrm{mg}) ;[\alpha]_{\mathrm{D}}{ }^{20}=-1.4\left(c=0.1 \mathrm{CHCl}_{3}\right) ;{ }^{1} \mathrm{H} \mathrm{NMR}(400 \mathrm{MHz}$, $\left.\mathrm{CDCl}_{3}\right) \delta 7.84(\mathrm{~d}, J=8.0 \mathrm{~Hz}, 2 \mathrm{H}), 7.33(\mathrm{t}, J=8 \mathrm{~Hz}, 4 \mathrm{H}), 7.18(\mathrm{~d}$, $J=8.0 \mathrm{~Hz}, 2 \mathrm{H}), 6.10(\mathrm{~s}, 1 \mathrm{H}), 5.32(\mathrm{~s}, 1 \mathrm{H}), 3.89(\mathrm{~s}, 1 \mathrm{H}), 2.47(\mathrm{~s}, 3 \mathrm{H})$, 2.36 (s, 3H), 2.02 (s, 3H); ${ }^{13} \mathrm{C}\left\{{ }^{1} \mathrm{H}\right\}$ NMR (100 MHz, $\left.\mathrm{CDCl}_{3}\right) \delta 198.2,169.4,145.1,138.1,133.7$, 131.5, 129.5, 129.0, 128.5, 126.8, 75.6, 75.0, 21.7, 21.0, 20.6; HRMS (ESI) m/z calcd for $\mathrm{C}_{19} \mathrm{H}_{20} \mathrm{O}_{4}[\mathrm{M}+\mathrm{Na}]^{+}: 335.1254$, found: 335.1247 ; HPLC analysis: 88\% ee (Chiralcel OD-H, 02:98 $\left.{ }^{\mathrm{i}} \mathrm{PrOH} / \mathrm{Hexane}, 1 \mathrm{~mL} / \mathrm{min}\right), \mathrm{R}_{\mathrm{t}}($ minor $)=24.6 \mathrm{~min}, \mathrm{R}_{\mathrm{t}}($ major $)=20.0 \mathrm{~min}$.

(1S,2R)-1-(4-Ethylphenyl)-2-hydroxy-3-oxo-3-(p-tolyl)propyl acetate (2m): Colorless gummy

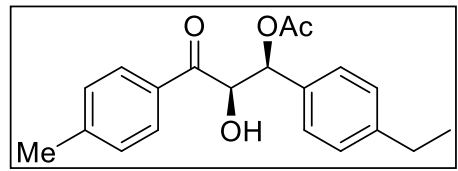
liquid, $69 \%$ yield $(22.5 \mathrm{mg}) ;[\alpha]_{\mathrm{D}}{ }^{20}=+31.4\left(c=0.1 \mathrm{CHCl}_{3}\right) ;{ }^{1} \mathrm{H}$ NMR (400 MHz, $\left.\mathrm{CDCl}_{3}\right) \delta 7.79(\mathrm{~d}, J=4.0 \mathrm{~Hz}, 2 \mathrm{H}), 7.33-7.27(\mathrm{~m}$, $4 \mathrm{H}), 7.17(\mathrm{~d}, J=8.0 \mathrm{~Hz}, 2 \mathrm{H}), 6.06(\mathrm{~d}, J=4.0 \mathrm{~Hz}, 1 \mathrm{H}), 5.30(\mathrm{~d}, J=$ $8.0 \mathrm{~Hz}, 1 \mathrm{H}), 3.84(\mathrm{~d}, J=8.0 \mathrm{~Hz}, 1 \mathrm{H}), 2.63(\mathrm{q}, J=8.0 \mathrm{~Hz}, 2 \mathrm{H}), 2.43$ (s, 3H), 1.99 (s, 3H), 1.22 (t, $J=8.0 \mathrm{~Hz}, 3 \mathrm{H}) ;{ }^{13} \mathrm{C}\left\{{ }^{1} \mathrm{H}\right\} \mathrm{NMR}\left(100 \mathrm{MHz}, \mathrm{CDCl}_{3}\right) \delta 198.2,169.5,145.1,144.5,133.9,131.6$, 129.6, 128.5, 127.9, 127.0, 75.7, 75.1, 28.5, 21.7, 20.7, 15.4; HRMS (ESI) $\mathrm{m} / \mathrm{z}$ calcd for $\mathrm{C}_{20} \mathrm{H}_{22} \mathrm{O}_{4} \mathrm{Na}[\mathrm{M}+\mathrm{Na}]^{+}: 349.1410$, found: 349.1411; HPLC analysis: 91\% ee (Chiralcel OD-H, 02:98 $\left.{ }^{i} \mathrm{PrOH} / \mathrm{Hexane}, 1 \mathrm{~mL} / \mathrm{min}\right), \mathrm{R}_{\mathrm{t}}($ minor $)=22.3 \mathrm{~min}, \mathrm{R}_{\mathrm{t}}($ major $)=18.7 \mathrm{~min}$.

(1S,2R)-1-(4-Bromophenyl)-2-hydroxy-3-oxo-3-( $p$-tolyl)propyl acetate (2n): Colorless gummy OAc liquid, 64\% yield $(24.2 \mathrm{mg}) ;[\alpha]_{\mathrm{D}}{ }^{20}=-14.4\left(c=0.1 \mathrm{CHCl}_{3}\right) ;{ }^{1} \mathrm{H} \mathrm{NMR}$ $\left(400 \mathrm{MHz}, \mathrm{CDCl}_{3}\right) \delta 7.79(\mathrm{~d}, J=8.0 \mathrm{~Hz}, 2 \mathrm{H}), 7.47(\mathrm{~d}, J=8.0 \mathrm{~Hz}$, $2 \mathrm{H}), 7.32(\mathrm{~d}, J=8.0 \mathrm{~Hz}, 2 \mathrm{H}), \quad 7.27(\mathrm{~d}, J=8.0 \mathrm{~Hz}, 2 \mathrm{H}), 6.02(\mathrm{~d}, J=$ $2.5 \mathrm{~Hz}, 1 \mathrm{H}), 5.25(\mathrm{dd}, J=8.0 \mathrm{~Hz}, 4.0 \mathrm{~Hz}, 1 \mathrm{H}), 3.88(\mathrm{~d}, J=8.0 \mathrm{~Hz}, 1 \mathrm{H}), 2.45(\mathrm{~s}, 3 \mathrm{H}), 1.99(\mathrm{~s}, 3 \mathrm{H})$; ${ }^{13} \mathrm{C}\left\{{ }^{1} \mathrm{H}\right\}$ NMR (100 MHz, $\left.\mathrm{CDCl}_{3}\right) \delta 197.7,169.3,145.5,135.7,131.5,131.3,129.7,128.7,128.5$, 122.5, 75.2, 74.7, 21.8, 20.6; HRMS (ESI) $\mathrm{m} / \mathrm{z}$ calcd. for $\mathrm{C}_{18} \mathrm{H}_{17} \mathrm{BrO}_{4} \mathrm{Na}[\mathrm{M}+\mathrm{Na}]^{+}$: 401.0182, found: 401.0177; HPLC analysis: 94\% ee (Chiralcel OZ-H, $10: 90{ }^{i} \mathrm{PrOH} / \mathrm{Hexane}, 1 \mathrm{~mL} / \mathrm{min}$ ), $\mathrm{R}_{\mathrm{t}}($ minor $)=34.4 \mathrm{~min}, \mathrm{R}_{\mathrm{t}}($ major $)=16.6 \mathrm{~min}$.

(1S,2R)-1-(4-Chlorophenyl)-2-hydroxy-3-oxo-3-(p-tolyl)propyl acetate (20): Colorless

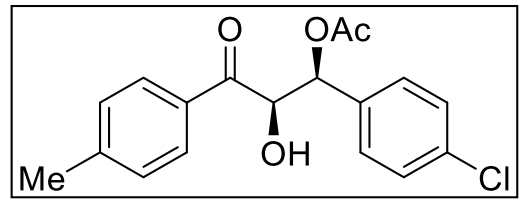
gummy liquid, $84 \%$ yield $(28 \mathrm{mg}) ;[\alpha]_{\mathrm{D}}{ }^{20}=-19.6(c=0.1$ $\left.\mathrm{CHCl}_{3}\right) ;{ }^{1} \mathrm{HNMR}\left(400 \mathrm{MHz}, \mathrm{CDCl}_{3}\right) \delta 7.79(\mathrm{~d}, J=8.0 \mathrm{~Hz}, 2 \mathrm{H})$, 7.33-7.30 (m, 6H), $6.03(\mathrm{~d}, J=4.0 \mathrm{~Hz}, 1 \mathrm{H}), 5.26(\mathrm{dd}, J=8.0$ $\mathrm{Hz}, 2.6 \mathrm{~Hz}, 1 \mathrm{H}), 3.88(\mathrm{~d}, J=8.0 \mathrm{~Hz}, 1 \mathrm{H}), 2.44(\mathrm{~s}, 3 \mathrm{H}), 1.99(\mathrm{~s}, 3 \mathrm{H}) ;{ }^{13} \mathrm{C}\left\{{ }^{1} \mathrm{H}\right\}$ NMR $(100 \mathrm{MHz}$, 
$\left.\mathrm{CDCl}_{3}\right) \delta 197.8,169.4,145.5,135.2,134.3,131.3,129.7,128.6,128.5,128.4,75.1,74.8,21.8$, 20.6; HRMS (ESI) $\mathrm{m} / \mathrm{z}$ calcd for $\mathrm{C}_{18} \mathrm{H}_{17} \mathrm{ClO}_{4} \mathrm{Na}[\mathrm{M}+\mathrm{Na}]^{+}: 355.0708$, found: 355.0704; HPLC analysis: 95\% ee (Chiralcel OZ-H, $\left.10: 90{ }^{~} \mathrm{PrOH} / \mathrm{Hexane}, 1 \mathrm{~mL} / \mathrm{min}\right), \mathrm{R}_{\mathrm{t}}($ minor $)=30.4 \mathrm{~min}$, $\mathrm{R}_{\mathrm{t}}($ major $)=15.3 \mathrm{~min}$.

(1S,2R)-3-(4-Bromophenyl)-2-hydroxy-3-oxo-1-( $m$-tolyl)propyl acetate $(2 \mathrm{p}):$ Colorless

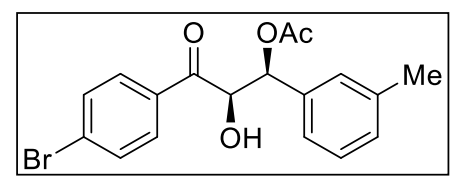

gummy liquid, 68\% yield $(25.7 \mathrm{mg}) ;[\alpha]_{\mathrm{D}}{ }^{20}=+32.2\left(c=0.1 \mathrm{CHCl}_{3}\right)$; ${ }^{1} \mathrm{H} \mathrm{NMR}\left(400 \mathrm{MHz}, \mathrm{CDCl}_{3}\right) \delta 7.74(\mathrm{~d}, J=8.0 \mathrm{~Hz}, 2 \mathrm{H}), 7.64(\mathrm{~d}, J=$ $8.0 \mathrm{~Hz}, 2 \mathrm{H}), 7.26-7.19(\mathrm{~m}, 1 \mathrm{H}), 7.17-7.09(\mathrm{~m}, 3 \mathrm{H}), 6.00(\mathrm{~d}, J=4.0$ $\mathrm{Hz}, 1 \mathrm{H}), 5.26(\mathrm{~s}, 1 \mathrm{H}), 3.74(\mathrm{~d}, J=4.0 \mathrm{~Hz}, 1 \mathrm{H}), 2.33(\mathrm{~s}, 3 \mathrm{H}), 2.02(\mathrm{~s}, 3 \mathrm{H}) ;{ }^{13} \mathrm{C}\left\{{ }^{1} \mathrm{H}\right\} \mathrm{NMR}(100$ $\left.\mathrm{MHz}, \mathrm{CDCl}_{3}\right) \delta 197.9,169.5,138.1,136.1,133.0,132.2,129.8,129.4,129.3,128.4,127.5,123.9$, 75.5, 75.3, 21.4, 20.7; HRMS (ESI) $\mathrm{m} / \mathrm{z}$ calcd. for $\mathrm{C}_{18} \mathrm{H}_{17} \mathrm{BrO}_{4} \mathrm{Na}[\mathrm{M}+\mathrm{Na}]^{+}$: 401.0182, found: 401.0184; HPLC analysis: 98\% ee (Chiralcel OD-H, 02:98 ${ }^{i} \mathrm{PrOH} / \mathrm{Hexane}, 1 \mathrm{~mL} / \mathrm{min}$ ), $\mathrm{R}_{\mathrm{t}}($ minor $)=40.9 \min , \mathrm{R}_{\mathrm{t}}($ major $)=26.4 \mathrm{~min}$.

(1S,2R)-3-(4-Bromophenyl)-2-hydroxy-3-oxo-1-(p-tolyl)propyl acetate (2q): Colorless

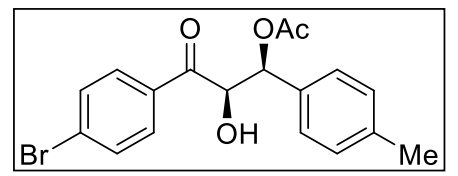
gummy liquid, $64 \%$ yield $(24.2 \mathrm{mg}) ;[\alpha]_{\mathrm{D}}{ }^{20}=+33.2\left(c=0.1 \mathrm{CHCl}_{3}\right)$; ${ }^{1} \mathrm{H}$ NMR $\left(400 \mathrm{MHz}, \mathrm{CDCl}_{3}\right) \delta 7.74(\mathrm{~d}, J=12.0 \mathrm{~Hz}, 2 \mathrm{H}), 7.65(\mathrm{~d}, J$ $=8.0 \mathrm{~Hz}, 2 \mathrm{H}), 7.25(\mathrm{~d}, J=8.0 \mathrm{~Hz}, 2 \mathrm{H}), 7.14(\mathrm{~d}, J=8.0 \mathrm{~Hz}, 3 \mathrm{H}), 6.01$ (d, $J=4.0 \mathrm{~Hz}, 1 \mathrm{H}), 5.25(\mathrm{dd}, J=8.0 \mathrm{~Hz}, 4.0 \mathrm{~Hz}, 1 \mathrm{H}), 3.70(\mathrm{~d}, J=8.0 \mathrm{~Hz}, 1 \mathrm{H}), 2.33(\mathrm{~s}, 3 \mathrm{H}), 2.01$ $(\mathrm{s}, 3 \mathrm{H}) ;{ }^{13} \mathrm{C}\left\{{ }^{1} \mathrm{H}\right\} \mathrm{NMR}\left(100 \mathrm{MHz}, \mathrm{CDCl}_{3}\right) \delta 198.0,169.5,138.5,133.2,133.0,132.2,129.9,129.4$, 129.1, 126.9, 75.5, 75.3, 21.1, 20.7; HRMS (ESI) m/z calcd. for $\mathrm{C}_{18} \mathrm{H}_{17} \mathrm{BrO}_{4} \mathrm{Na}[\mathrm{M}+\mathrm{Na}]^{+}$: 401.0182, found: 401.0183; HPLC analysis: 91\% ee (Chiralcel IC, 02:98 ${ }^{i} \mathrm{PrOH} / \mathrm{Hexane}, 1$ $\mathrm{mL} / \mathrm{min}), \mathrm{R}_{\mathrm{t}}($ minor $)=32.7 \mathrm{~min}, \mathrm{R}_{\mathrm{t}}($ major $)=25.2 \mathrm{~min}$.

(1S,2R)-3-(4-Bromophenyl)-1-(4-ethylphenyl)-2-hydroxy-3-oxopropyl acetate (2r): Colorless (n) gummy liquid, $81 \%$ yield $(31.6 \mathrm{mg}) ;[\alpha]_{\mathrm{D}}{ }^{20}=-14.6\left(c=0.1 \mathrm{CHCl}_{3}\right)$; ${ }^{1} \mathrm{H}$ NMR $\left(400 \mathrm{MHz}, \mathrm{CDCl}_{3}\right) \delta 7.72(\mathrm{~d}, J=8.0 \mathrm{~Hz}, 2 \mathrm{H}), 7.63(\mathrm{~d}, J=$ $8.0 \mathrm{~Hz}, 2 \mathrm{H}), 7.27(\mathrm{~d}, J=8.0 \mathrm{~Hz}, 2 \mathrm{H}), 7.16(\mathrm{~d}, J=8.0 \mathrm{~Hz}, 2 \mathrm{H}), 6.01$ $(\mathrm{d}, J=4.0 \mathrm{~Hz}, 1 \mathrm{H}), 5.25(\mathrm{dd}, J=8.0 \mathrm{~Hz}, 4.0 \mathrm{~Hz}, 1 \mathrm{H}), 3.72(\mathrm{~d}, J=8.0 \mathrm{~Hz}, 1 \mathrm{H}), 2.62(\mathrm{q}, J=8.0$ $\mathrm{Hz}, 2 \mathrm{H}), 2.01(\mathrm{~s}, 3 \mathrm{H}), 1.21(\mathrm{t}, J=8.0 \mathrm{~Hz}, 3 \mathrm{H}) ;{ }^{13} \mathrm{C}\left\{{ }^{1} \mathrm{H}\right\} \mathrm{NMR}\left(100 \mathrm{MHz}, \mathrm{CDCl}_{3}\right) \delta 198.0,169.6$, 144.8, 133.4, 133.0, 132.2, 129.9, 129.3, 128.0, 127.0, 75.5, 75.3, 28.5 20.7, 15.4; HRMS (ESI) calcd. for $\mathrm{C}_{19} \mathrm{H}_{19} \mathrm{BrO}_{4} \mathrm{Na}[\mathrm{M}+\mathrm{Na}]^{+}$: 413.0359, found: 413.0361; HPLC analysis: 99\% ee $\left(\right.$ Chiralcel OD-H, 02:98 $\left.{ }^{i} \mathrm{PrOH} / \mathrm{Hexane}, 1 \mathrm{~mL} / \mathrm{min}\right), \mathrm{R}_{\mathrm{t}}($ minor $)=28.4 \mathrm{~min}, \mathrm{R}_{\mathrm{t}}($ major $)=23.8 \mathrm{~min}$. 
(1S,2R)-3-(4-Bromophenyl)-2-hydroxy-1-(4-isopropylphenyl)-3-oxopropyl acetate (2s):

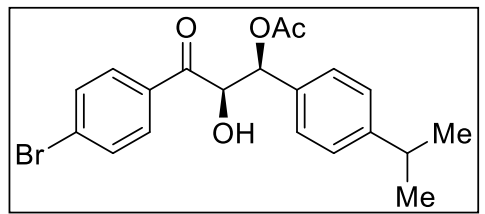

Colorless gummy liquid, $64 \%$ yield $(26 \mathrm{mg}) ;[\alpha]_{\mathrm{D}}{ }^{20}=+29.4(c=$ $\left.0.1 \mathrm{CHCl}_{3}\right) ;{ }^{1} \mathrm{H} \mathrm{NMR}\left(400 \mathrm{MHz}, \mathrm{CDCl}_{3}\right) \delta 7.71(\mathrm{~d}, J=8.0 \mathrm{~Hz}$, 2H), $7.61(\mathrm{~d}, J=8.0 \mathrm{~Hz}, 2 \mathrm{H}), 7.27(\mathrm{~d}, J=8.0 \mathrm{~Hz}, 2 \mathrm{H}), 7.17(\mathrm{~d}, J$ $=8.0 \mathrm{~Hz}, 2 \mathrm{H}), 6.00(\mathrm{~d}, J=4.0 \mathrm{~Hz}, 1 \mathrm{H}), 5.25(\mathrm{~d}, J=4.0 \mathrm{~Hz}, 1 \mathrm{H}), 3.72(\mathrm{~d}, J=8.0 \mathrm{~Hz}, 1 \mathrm{H}), 2.91-$ $2.81(\mathrm{~m}, 1 \mathrm{H}), 2.02(\mathrm{~s}, 3 \mathrm{H}), 1.22(\mathrm{~d}, J=8.0 \mathrm{~Hz}, 6 \mathrm{H}) ;{ }^{13} \mathrm{C}\left\{{ }^{1} \mathrm{H}\right\} \mathrm{NMR}\left(100 \mathrm{MHz}, \mathrm{CDCl}_{3}\right) \delta 198.1$, 169.6, 149.4, 133.6, 133.0, 132.1, 129.8, 129.3, 127.0, 126.5, 75.6, 75.3, 33.8, 23.8, 20.7; HRMS (ESI) $\mathrm{m} / \mathrm{z}$ calcd. for $\mathrm{C}_{20} \mathrm{H}_{21} \mathrm{BrO}_{4} \mathrm{Na}[\mathrm{M}+\mathrm{Na}]^{+}: 429.0495$, found: 429.0491 ; HPLC analysis: $95 \%$ $e e\left(\right.$ Chiralcel IC, $\left.02: 98{ }^{\mathrm{i}} \mathrm{PrOH} / \mathrm{Hexane} 0.7 \mathrm{~mL} / \mathrm{min}\right), \mathrm{R}_{\mathrm{t}}($ minor $)=98.1 \mathrm{~min}, \mathrm{R}_{\mathrm{t}}$ (major) $=92.1 \mathrm{~min}$. (1S,2R)-3-(4-Chlorophenyl)-2-hydroxy-3-oxo-1-( $m$-tolyl)propyl acetate (2t): Colorless

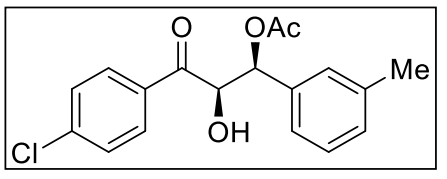

gummy liquid, $81 \%$ yield $(27 \mathrm{mg}) ;[\alpha]_{\mathrm{D}}{ }^{20}=-19.4\left(c=0.1 \mathrm{CHCl}_{3}\right)$; ${ }^{1} \mathrm{H}$ NMR (400 MHz, $\left.\mathrm{CDCl}_{3}\right) \delta 7.82(\mathrm{~d}, J=8.0 \mathrm{~Hz}, 2 \mathrm{H}), 7.47(\mathrm{~d}, J$ $=12.0 \mathrm{~Hz}, 2 \mathrm{H}), 7.27-7.20(\mathrm{~m}, 1 \mathrm{H}), 7.17-7.09(\mathrm{~m}, 3 \mathrm{H}), 6.01(\mathrm{~d}, J=$ $4.0 \mathrm{~Hz}, 1 \mathrm{H}), 5.26(\mathrm{dd}, J=8.0 \mathrm{~Hz}, 4.0 \mathrm{~Hz}, 1 \mathrm{H}), 3.73(\mathrm{~d}, J=8.0 \mathrm{~Hz}, 1 \mathrm{H}), 2.33(\mathrm{~s}, 3 \mathrm{H}), 2.02$ (s, 3H); ${ }^{13} \mathrm{C}\left\{{ }^{1} \mathrm{H}\right\} \mathrm{NMR}\left(100 \mathrm{MHz}, \mathrm{CDCl}_{3}\right) \delta 197.7,169.5,140.6,138.1,136.2,132.6,129.8,129.3,129.2$, 128.4, 127.6, 123.9, 75.6, 75.3, 21.4, 20.7; HRMS (ESI) m/z calcd for $\mathrm{C}_{18} \mathrm{H}_{17} \mathrm{ClO}_{4} \mathrm{Na}[\mathrm{M}+\mathrm{Na}]^{+}$: 355.0708, found: 355.0708; HPLC analysis: 94\% ee (Chiralcel OD-H, 02:98 $\mathrm{PrOH} / \mathrm{Hexane}, 1$ $\mathrm{mL} /$ min $), \mathrm{R}_{\mathrm{t}}($ minor $)=37.3 \min , \mathrm{R}_{\mathrm{t}}($ major $)=23.8 \mathrm{~min}$.

(1S,2R)-3-(4-Chlorophenyl)-2-hydroxy-3-oxo-1-(p-tolyl)propyl acetate $(2 \mathrm{u}):$ Colorless

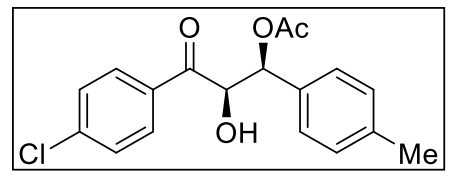

gummy liquid, $73 \%$ yield $(24.2 \mathrm{mg}) ;[\alpha]_{\mathrm{D}}{ }^{20}=+38.4\left(c=0.1 \mathrm{CHCl}_{3}\right)$; ${ }^{1} \mathrm{H}$ NMR $\left(400 \mathrm{MHz}, \mathrm{CDCl}_{3}\right) \delta 7.82(\mathrm{~d}, J=8.0 \mathrm{~Hz}, 2 \mathrm{H}), 7.47(\mathrm{~d}, J=$ $12.0 \mathrm{~Hz}, 2 \mathrm{H}), 7.23(\mathrm{~d}, J=8.0 \mathrm{~Hz}, 2 \mathrm{H}), 7.14(\mathrm{~d}, J=8.0 \mathrm{~Hz}, 2 \mathrm{H}), 6.02$ (d, $J=3.0 \mathrm{~Hz}, 1 \mathrm{H}), 5.24(\mathrm{~d}, J=4.0 \mathrm{~Hz}, 1 \mathrm{H}), 3.72(\mathrm{~d}, J=8.0 \mathrm{~Hz}, 1 \mathrm{H}), 2.33(\mathrm{~s}, 3 \mathrm{H}), 2.00(\mathrm{~s}, 3 \mathrm{H})$; ${ }^{13} \mathrm{C}\left\{{ }^{1} \mathrm{H}\right\}$ NMR (100 MHz, $\left.\mathrm{CDCl}_{3}\right) \delta 197.7,169.5,140.6,138.4,133.2,132.6,129.8,129.2,129.1$, 126.9, 75.5, 75.3, 21.1, 20.7; HRMS (ESI) $\mathrm{m} / \mathrm{z}$ calcd. for $\mathrm{C}_{18} \mathrm{H}_{17} \mathrm{ClO}_{4} \mathrm{Na}[\mathrm{M}+\mathrm{Na}]^{+}$: 355.0708, found: 355.0707; HPLC analysis: 96\% ee (Chiralcel OD-H, 02:98 ${ }^{i} \mathrm{PrOH} / \mathrm{Hexane}, 1 \mathrm{~mL} / \mathrm{min}$ ), $\mathrm{R}_{\mathrm{t}}($ minor $)=31.2 \min , \mathrm{R}_{\mathrm{t}}($ major $)=23.0 \mathrm{~min}$.

(1S,2R)-3-(4-Chlorophenyl)-1-(4-ethylphenyl)-2-hydroxy-3-oxopropyl acetate (2v): (O) Colorless gummy liquid, 76\% yield $(26.3 \mathrm{mg}) ;[\alpha]_{\mathrm{D}}{ }^{20}=+34.4(c=$ $\left.0.1 \mathrm{CHCl}_{3}\right) ;{ }^{1} \mathrm{H} \mathrm{NMR}\left(400 \mathrm{MHz}, \mathrm{CDCl}_{3}\right) \delta 7.81(\mathrm{~d}, J=8.0 \mathrm{~Hz}, 2 \mathrm{H})$, $7.46(\mathrm{~d}, J=8.0 \mathrm{~Hz}, 2 \mathrm{H}), 7.27(\mathrm{~d}, J=8.0 \mathrm{~Hz}, 2 \mathrm{H}), 7.16(\mathrm{~d}, J=8.0$ 
$\mathrm{Hz}, 2 \mathrm{H}), 6.01(\mathrm{~d}, J=4.0 \mathrm{~Hz} 1 \mathrm{H}), 5.26(\mathrm{dd}, J=8.0 \mathrm{~Hz}, 4.0 \mathrm{~Hz}, 1 \mathrm{H}), 3.72(\mathrm{~d}, J=8.0 \mathrm{~Hz} 1 \mathrm{H}), 2.62$ $(\mathrm{q}, J=8.0 \mathrm{~Hz}, 2 \mathrm{H}), 2.01(\mathrm{~s}, 3 \mathrm{H}), 1.21(\mathrm{t}, J=8.0 \mathrm{~Hz}) ;{ }^{13} \mathrm{C}\left\{{ }^{1} \mathrm{H}\right\} \mathrm{NMR}\left(100 \mathrm{MHz}, \mathrm{CDCl}_{3}\right) \delta 197.8$, 169.6, 144.8, 140.6, 133.5, 132.6, 129.8, 129.2, 128.0, 127.0, 75.6, 75.3, 28.5, 20.7, 15.4; HRMS (ESI) $\mathrm{m} / \mathrm{z}$ calcd. for $\mathrm{C}_{19} \mathrm{H}_{19} \mathrm{ClO}_{4} \mathrm{Na}[\mathrm{M}+\mathrm{Na}]^{+}: 369.0864$, found: 369.0865 ; HPLC analysis: $94 \%$ ee (Chiralcel OD-H, 02:98 $\left.{ }^{\mathrm{i}} \mathrm{PrOH} / \mathrm{Hexane}, 1 \mathrm{~mL} / \mathrm{min}\right), \mathrm{R}_{\mathrm{t}}($ minor $)=26.4 \mathrm{~min}, \mathrm{R}_{\mathrm{t}}($ major $)=$ $22.0 \mathrm{~min}$.

(1S,2R)-3-(4-Chlorophenyl)-2-hydroxy-1-(4-isopropylphenyl)-3-oxopropyl acetate (2w):

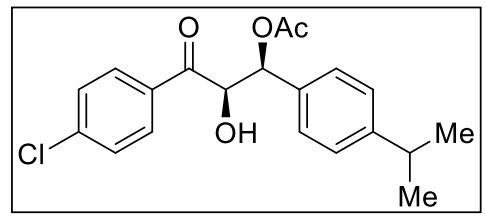
Colorless gummy liquid, $75 \%$ yield $(27 \mathrm{mg}) ;[\alpha]_{\mathrm{D}}{ }^{20}=+24.0(c=$ $\left.0.1 \mathrm{CHCl}_{3}\right) ;{ }^{1} \mathrm{H} \mathrm{NMR}\left(400 \mathrm{MHz}, \mathrm{CDCl}_{3}\right) \delta 7.79(\mathrm{~d}, J=8.0 \mathrm{~Hz}$, 2H), $7.44(\mathrm{~d}, J=8.0 \mathrm{~Hz}, 2 \mathrm{H}), 7.30-7.24(\mathrm{~m}, 2 \mathrm{H}), 7.17(\mathrm{~d}, J=8.0$

$\mathrm{Hz}, 2 \mathrm{H}), 6.0(\mathrm{~d}, J=4.0 \mathrm{~Hz}, 1 \mathrm{H}), 5.30-5.23(\mathrm{~m}, 1 \mathrm{H}), 3.73(\mathrm{~d}, J=8.0 \mathrm{~Hz}, 1 \mathrm{H}), 2.92-2.82(\mathrm{~m}, 1 \mathrm{H})$, $2.02(\mathrm{~s}, 3 \mathrm{H}), 1.22(\mathrm{~d}, J=8.0 \mathrm{~Hz}, 6 \mathrm{H}) ;{ }^{13} \mathrm{C}\left\{{ }^{1} \mathrm{H}\right\} \mathrm{NMR}\left(100 \mathrm{MHz}, \mathrm{CDCl}_{3}\right) \delta 197.9,169.6,149.4$, $140.5,133.6,132.6,129.8,129.2,127.0,126.5,75.6,75.3,33.8,23.8,20.7$; HMS (ESI) m/z calcd. for $\mathrm{C}_{20} \mathrm{H}_{21} \mathrm{ClO}_{4} \mathrm{Na}[\mathrm{M}+\mathrm{Na}]^{+}$: 383.1021, found: 383.1021; HPLC analysis: 93\% ee (Chiralcel IC, 02:98 ${ }^{i} \mathrm{PrOH} /$ Hexane, $\left.1 \mathrm{~mL} / \mathrm{min}\right), \mathrm{R}_{\mathrm{t}}($ minor $)=42.1 \mathrm{~min}, \mathrm{R}_{\mathrm{t}}($ major $)=46.3 \mathrm{~min}$.

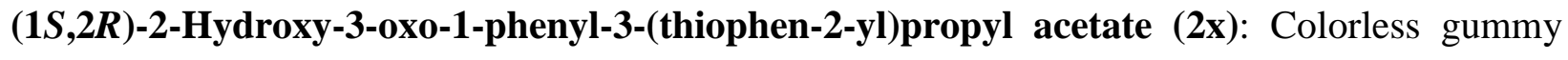
ST

liquid, $81 \%$ yield $(23.5 \mathrm{mg}) ;[\alpha]_{\mathrm{D}}{ }^{20}=-59.4\left(c=0.1 \mathrm{CHCl}_{3}\right) ;{ }^{1} \mathrm{H}$ NMR $(400$ $\left.\mathrm{MHz} \mathrm{CDCl}_{3}\right) \delta 7.76(\mathrm{t}, J=4.0 \mathrm{~Hz}, 2 \mathrm{H}), 7.42-7.24(\mathrm{~m}, 6 \mathrm{H}), 7.17(\mathrm{t}, J=8.0$ $\mathrm{Hz}, 1 \mathrm{H}), 6.16$ (d, $J=4.0 \mathrm{~Hz}, 1 \mathrm{H}), 5.09$ (dd, $J=8.0 \mathrm{~Hz}, 4.0 \mathrm{~Hz}, 1 \mathrm{H}), 3.64$ (d, $J=8.0 \mathrm{~Hz}, 1 \mathrm{H}), 2.06(\mathrm{~s}, 1 \mathrm{H}) ;{ }^{13} \mathrm{C}\left\{{ }^{1} \mathrm{H}\right\} \mathrm{NMR}\left(100 \mathrm{MHz}, \mathrm{CDCl}_{3}\right) \delta 190.7,169.6,140.1,136.4$, 135.3, 133.2, 128.5, 128.5, 128.4, 126.9, 76.4, 76.2, 20.8; HRMS (ESI) $\mathrm{m} / \mathrm{z}$ calcd. for $\mathrm{C}_{15} \mathrm{H}_{14} \mathrm{O}_{4} \mathrm{SNa}[\mathrm{M}+\mathrm{Na}]^{+}:$313.0505, found: 313.0502; HPLC analysis: 95\% ee(Chiralcel OD-H, 02:98 ${ }^{i} \mathrm{PrOH} /$ Hexane, $\left.1 \mathrm{~mL} / \mathrm{min}\right), \mathrm{R}_{\mathrm{t}}($ minor $)=74.8 \mathrm{~min}, \mathrm{R}_{\mathrm{t}}($ major $)=52.3 \mathrm{~min}$.

(1S,2R)-2-Hydroxy-3-(naphthalen-2-yl)-3-oxo-1-phenylpropyl acetate (2y): Colorless (OAC gummy liquid, $78 \%$ yield $(26 \mathrm{mg}) ;[\alpha]_{\mathrm{D}}{ }^{20}=+26.0\left(c=0.1 \mathrm{CHCl}_{3}\right) ;{ }^{1} \mathrm{H}$ NMR (400 MHz, $\left.\mathrm{CDCl}_{3}\right) \delta 8.41(\mathrm{~s}, 1 \mathrm{H}), 8.00-7.87(\mathrm{~m}, 4 \mathrm{H}), 7.68-7.56$ $(\mathrm{m}, 2 \mathrm{H}), 7.42(\mathrm{~d}, J=8.0 \mathrm{~Hz}, 2 \mathrm{H}), 7.38-7.27(\mathrm{~m}, 3 \mathrm{H}), 6.16(\mathrm{~d}, J=4.0 \mathrm{~Hz}$,

$1 \mathrm{H}), 5.51(\mathrm{~s}, 1 \mathrm{H}), 3.91(\mathrm{~s}, 1 \mathrm{H}), 2.00(\mathrm{~s}, 3 \mathrm{H}) ;{ }^{13} \mathrm{C}\left\{{ }^{1} \mathrm{H}\right\} \mathrm{NMR}\left(100 \mathrm{MHz}, \mathrm{CDCl}_{3}\right) \delta$ 198.6, 169.5, $136.5,135.9,132.3,131.5,130.5,129.6,129.0,128.9$, 128.5, 128.4, 127.8, 127.1, 127.0, 123.7, 75.87, 75.1, 20.7; HRMS (ESI) m/z calcd for $\mathrm{C}_{21} \mathrm{H}_{18} \mathrm{O}_{4} \mathrm{Na}[\mathrm{M}+\mathrm{Na}]^{+}: 357.1097$, found: 357.1096; 
HPLC analysis: 94\% ee (Chiralcel OD-H, 02:98 $\left.{ }^{i} \mathrm{PrOH} / \mathrm{Hexane}, 1 \mathrm{~mL} / \mathrm{min}\right), \mathrm{R}_{\mathrm{t}}($ minor $)=56.5$ $\min , \mathrm{R}_{\mathrm{t}}($ major $)=66.3 \mathrm{~min}$.

(1S,2R)-2-Hydroxy-1-(naphthalen-1-yl)-3-oxo-3-phenylpropyl acetate (2z): Colorless gummy

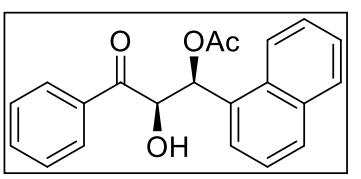
liquid, $73 \%$ yield $(24.4 \mathrm{mg}) ;[\alpha]_{\mathrm{D}}{ }^{20}=+15.6\left(c=0.1 \mathrm{CHCl}_{3}\right) ;{ }^{1} \mathrm{H} \mathrm{NMR}$ $\left(400 \mathrm{MHz}, \mathrm{CDCl}_{3}\right) \delta 8.09(\mathrm{~d}, J=8.0 \mathrm{~Hz}, 1 \mathrm{H}), 7.87-7.82(\mathrm{~m}, 3 \mathrm{H}), 7.79(\mathrm{~d}$, $J=8.0 \mathrm{~Hz}, 1 \mathrm{H}), 7.68-7.54(\mathrm{~m}, 3 \mathrm{H}), 7.53-7.42(\mathrm{~m}, 4 \mathrm{H}), 6.81(\mathrm{~d}, J=3.1 \mathrm{~Hz}$, $1 \mathrm{H}), 5.47(\mathrm{dd}, J=8.0 \mathrm{~Hz}, 4.0 \mathrm{~Hz}, 1 \mathrm{H}), 3.86(\mathrm{~d}, J=8.0 \mathrm{~Hz}, 1 \mathrm{H}), 2.07(\mathrm{~s}, 3 \mathrm{H}) ;{ }^{13} \mathrm{C}\left\{{ }^{1} \mathrm{H}\right\} \mathrm{NMR}(100$ $\left.\mathrm{MHz}, \mathrm{CDCl}_{3}\right) \delta 199.5,169.5,134.4,134.0,133.7,132.2,130.2,129.1,129.0,128.8,128.2,126.6$, 125.7, 125.6, 125.2, 122.5, 74.6, 72.3, 20.7; HRMS (ESI) m/z calcd for $\mathrm{C}_{21} \mathrm{H}_{18} \mathrm{O}_{4} \mathrm{Na}[\mathrm{M}+\mathrm{Na}]^{+}$: 357.1097, found: 357.1094. HPLC analysis: Chiral separation was not achieved.

(1S,2R,E)-2-Hydroxy-3-oxo-1,5-diphenylpent-4-en-1-yl acetate (2aa): Colorless gummy

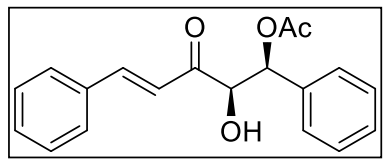
liquid, $62 \%$ yield $(19.2 \mathrm{mg}) ;[\alpha]_{\mathrm{D}}{ }^{20}=+86.8\left(c=0.1 \mathrm{CHCl}_{3}\right) ;{ }^{1} \mathrm{H} \mathrm{NMR}$ $\left(400 \mathrm{MHz}, \mathrm{CDCl}_{3}\right) \delta 7.72(\mathrm{~d}, J=15.8 \mathrm{~Hz}, 1 \mathrm{H}), 7.53(\mathrm{~d}, J=7.5 \mathrm{~Hz}$, 2H), $7.43(\mathrm{~d}, J=6.1 \mathrm{~Hz}, 4 \mathrm{H}), 7.39-7.31(\mathrm{~m}, 3 \mathrm{H}), 6.82(\mathrm{~d}, J=15.8 \mathrm{~Hz}, 1 \mathrm{H}), 6.15(\mathrm{~d}, J=3.8 \mathrm{~Hz}$, $1 \mathrm{H}), 4.70(\mathrm{~s}, 1 \mathrm{H}), 3.75(\mathrm{~d}, J=5.9 \mathrm{~Hz}, 1 \mathrm{H}), 2.10(\mathrm{~s}, 3 \mathrm{H}) ;{ }^{13} \mathrm{C}\left\{{ }^{1} \mathrm{H}\right\} \mathrm{NMR}\left(100 \mathrm{MHz}, \mathrm{CDCl}_{3}\right) \delta$ 196.9, 169.6, 145.0, 136.3, 133.9, 131.2, 129.0, 128.7, 128.5, 128.5, 127.0, 120.7, 78.1, 75.4, 20.9; HRMS (ESI): calcd for $\mathrm{C}_{19} \mathrm{H}_{18} \mathrm{O}_{4}(\mathrm{M}+\mathrm{Na}$ ): 333.1097, found: 333.1095; HPLC analysis: $98 \%$ ee (Chiralcel OD-H, 02:98 $\left.{ }^{i} \mathrm{PrOH} / \mathrm{Hexane}, 1 \mathrm{~mL} / \mathrm{min}\right), \mathrm{R}_{\mathrm{t}}($ minor $)=29.1 \mathrm{~min}, \mathrm{R}_{\mathrm{t}}($ major $)=27.6$ $\min$.

(1S,2R)-2-Hydroxy-3-oxo-1,3-diphenylpropyl propionate (2ab): Colorless gummy liquid,

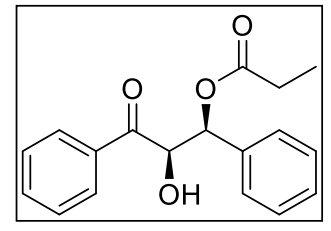
$66 \%$ yield $(19.7 \mathrm{mg}) ;[\alpha]_{\mathrm{D}}{ }^{20}=-25.8\left(c=0.1 \mathrm{CHCl}_{3}\right) ;{ }^{1} \mathrm{H} \mathrm{NMR}(400 \mathrm{MHz}$, $\left.\mathrm{CDCl}_{3}\right) \delta 7.90(\mathrm{~d}, J=7.8 \mathrm{~Hz}, 2 \mathrm{H}), 7.64(\mathrm{t}, J=7.5 \mathrm{~Hz}, 1 \mathrm{H}), 7.51(\mathrm{t}, J=7.6$ $\mathrm{Hz}, 2 \mathrm{H}), 7.40-7.29(\mathrm{~m}, 5 \mathrm{H}), 6.09(\mathrm{~d}, J=2.6 \mathrm{~Hz}, 1 \mathrm{H}), 5.34(\mathrm{~s}, 1 \mathrm{H}), 3.81$ (s, $1 \mathrm{H}), 2.28(\mathrm{q}, J=7.5 \mathrm{~Hz}, 2 \mathrm{H}), 1.05(\mathrm{t}, J=7.6 \mathrm{~Hz}, 3 \mathrm{H}) ;{ }^{13} \mathrm{C}\left\{{ }^{1} \mathrm{H}\right\} \mathrm{NMR}\left(100 \mathrm{MHz}, \mathrm{CDCl}_{3}\right) \delta 198.7$, 172.8, 136.6, 134.2, 134.1, 128.9, 128.4, 128.4, 126.9, 75.4, 75.2, 27.4, 8.9; HRMS (ESI): calcd for $\mathrm{C}_{18} \mathrm{H}_{18} \mathrm{O}_{4}(\mathrm{M}+\mathrm{Na}): 321.1097$, found: 321.1097; HPLC analysis: 93\% ee (Chiralcel OD-H, 02:98 ${ }^{i} \mathrm{PrOH} /$ Hexane, $\left.1 \mathrm{~mL} / \mathrm{min}\right), \mathrm{R}_{\mathrm{t}}($ minor $)=27.5 \mathrm{~min}, \mathrm{R}_{\mathrm{t}}($ major $)=20.3 \mathrm{~min}$. 
(1S,2R)-2-Hydroxy-3-oxo-1,3-diphenylpropyl pentanoate (2ac): Colorless gummy liquid, 60\%

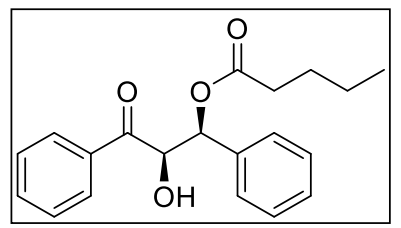
yield $(19.6 \mathrm{mg}) ;[\alpha]_{\mathrm{D}}{ }^{20}=-6.4\left(c=0.1 \mathrm{CHCl}_{3}\right) ;{ }^{1} \mathrm{H} \mathrm{NMR}(400 \mathrm{MHz}$, $\left.\mathrm{CDCl}_{3}\right) \delta 7.90(\mathrm{~d}, J=7.9 \mathrm{~Hz}, 2 \mathrm{H}), 7.64(\mathrm{t}, J=7.5 \mathrm{~Hz}, 1 \mathrm{H}), 7.52(\mathrm{t}, J=$ $7.6 \mathrm{~Hz}, 2 \mathrm{H}), 7.40-7.29(\mathrm{~m}, 5 \mathrm{H}), 6.10(\mathrm{~d}, J=2.5 \mathrm{~Hz}, 1 \mathrm{H}), 5.34(\mathrm{~s}, 1 \mathrm{H})$, $3.83(\mathrm{~d}, J=5.5 \mathrm{~Hz}, 1 \mathrm{H}), 2.25(\mathrm{t}, J=7.5 \mathrm{~Hz}, 2 \mathrm{H}), 1.55-1.45(\mathrm{~m}, 2 \mathrm{H}), 1.28-1.22(\mathrm{~m}, 2 \mathrm{H}), 0.87(\mathrm{t}, J$ $=7.3 \mathrm{~Hz}, 3 \mathrm{H}) ;{ }^{13} \mathrm{C}\left\{{ }^{1} \mathrm{H}\right\} \mathrm{NMR}\left(100 \mathrm{MHz}, \mathrm{CDCl}_{3}\right) \delta 198.6,172.2,136.7,134.1,134.1,128.9,128.4$, 128.4, 126.9, 75.3, 75.3, 33.8, 26.8, 22.0, 13.6; HRMS (ESI): calcd for $\mathrm{C}_{20} \mathrm{H}_{22} \mathrm{O}_{4}(\mathrm{M}+\mathrm{Na})$ : 349.1410, found: 349.1416; HPLC analysis: 90\% ee (Chiralcel OD-H, 02:98 ${ }^{i} \mathrm{PrOH} / \mathrm{Hexane}, 1$ $\mathrm{mL} / \mathrm{min}), \mathrm{R}_{\mathrm{t}}($ minor $)=22.0 \min , \mathrm{R}_{\mathrm{t}}($ major $)=17.2 \mathrm{~min}$.

\section{VII. ${ }^{1} \mathrm{H}$ and ${ }^{13} \mathrm{C}\left\{{ }^{1} \mathrm{H}\right\}$ spectra of $\mathbf{H y d r o x y}-0 x y a c y l a t i o n$ products 2:}




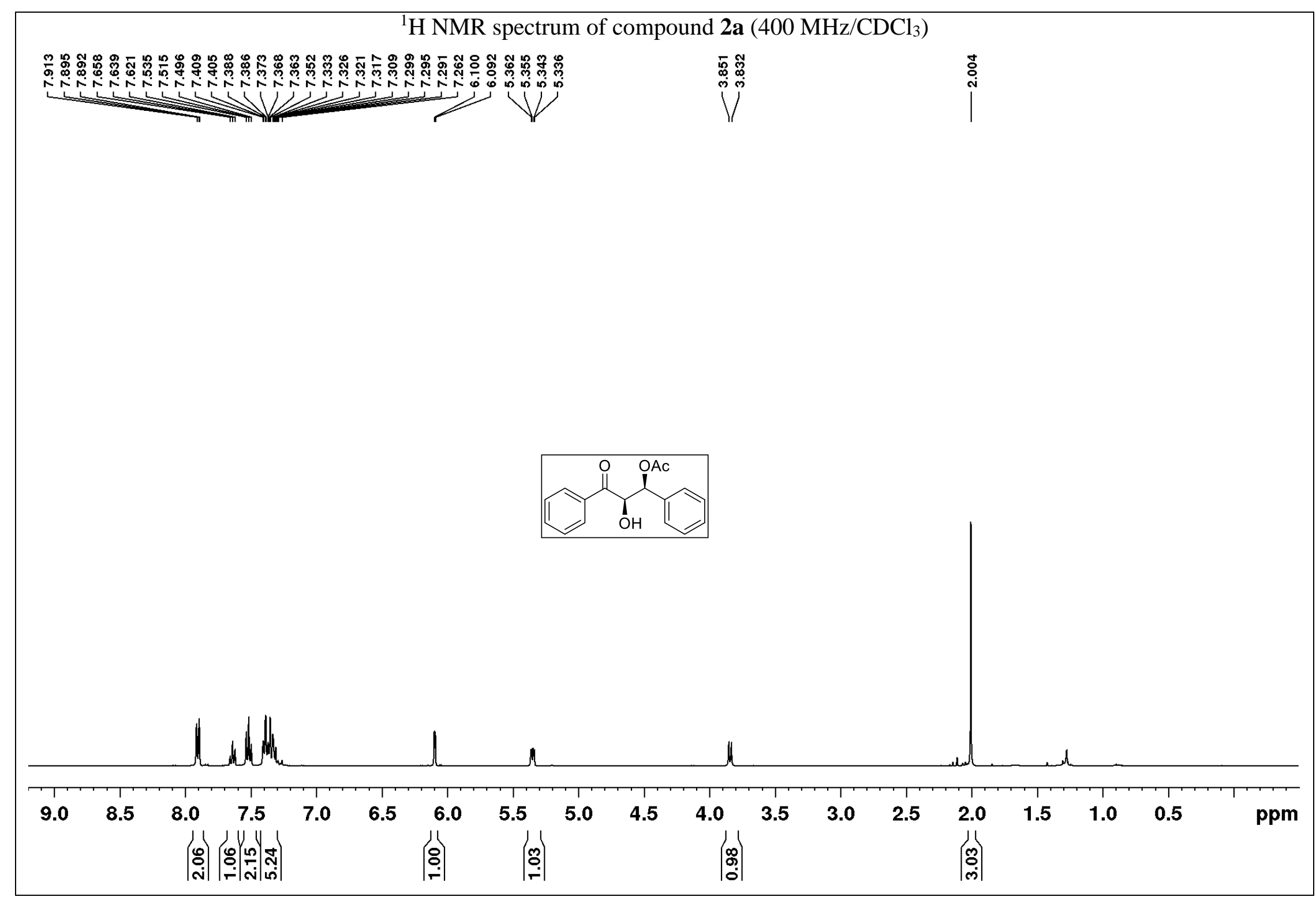




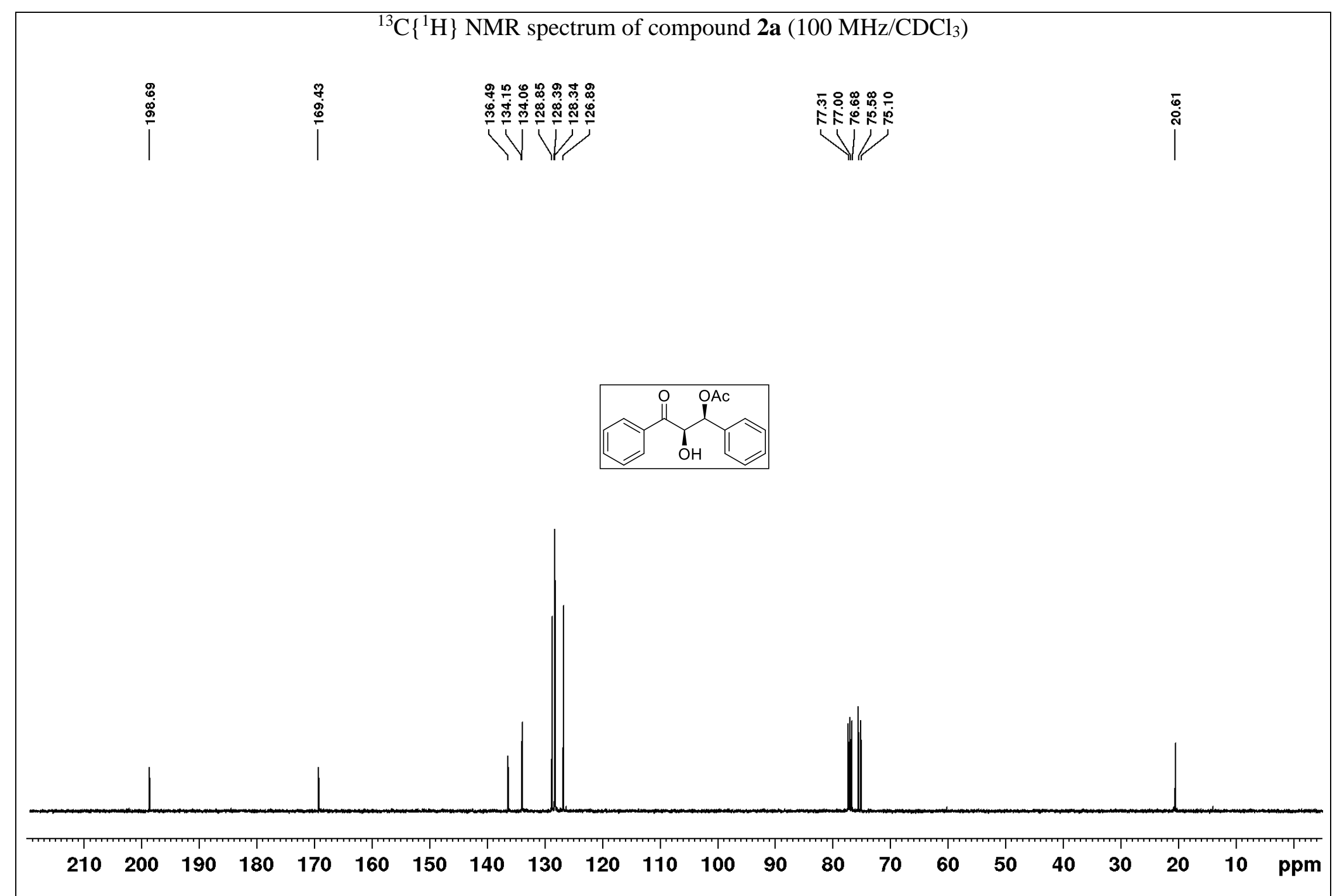




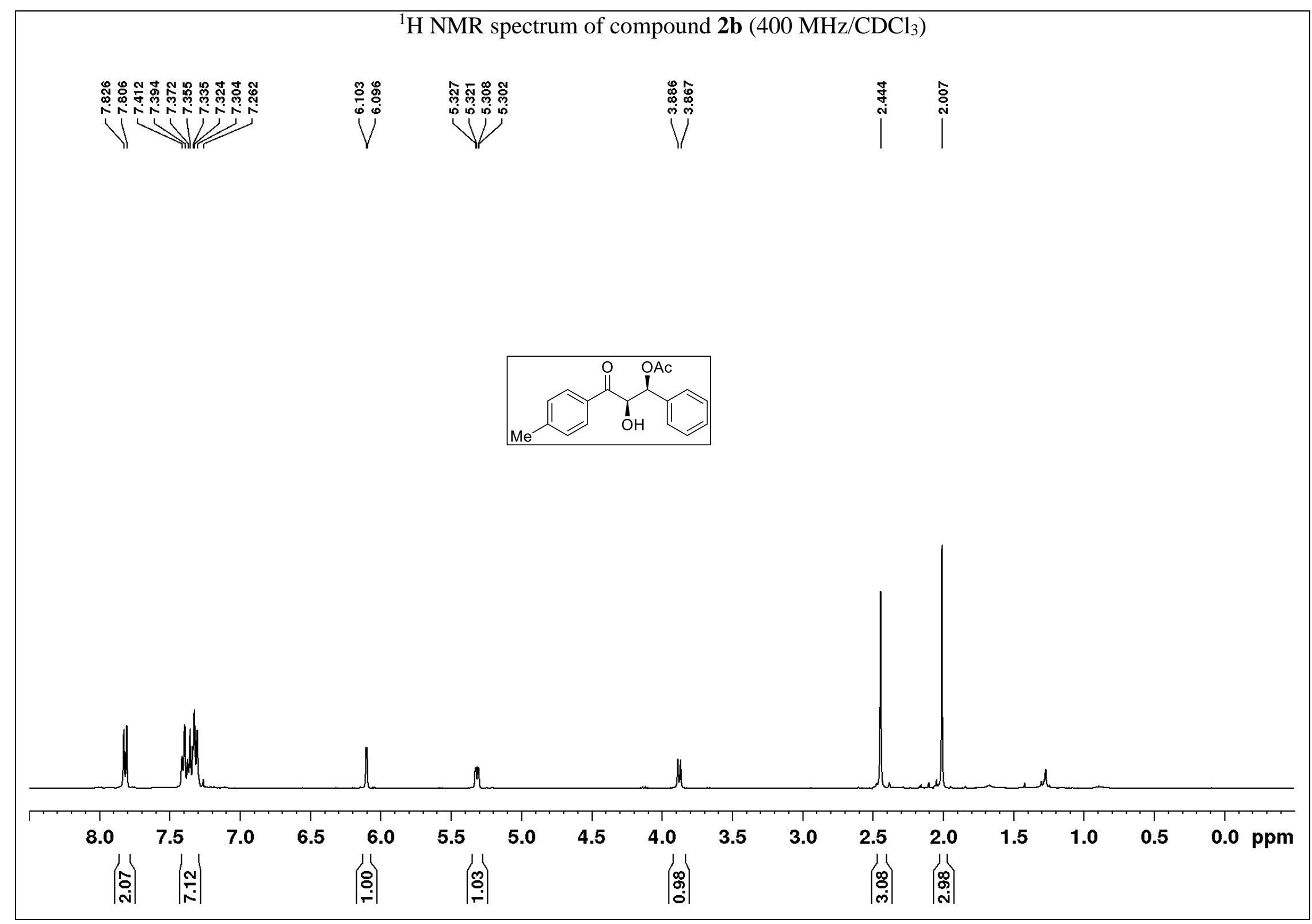




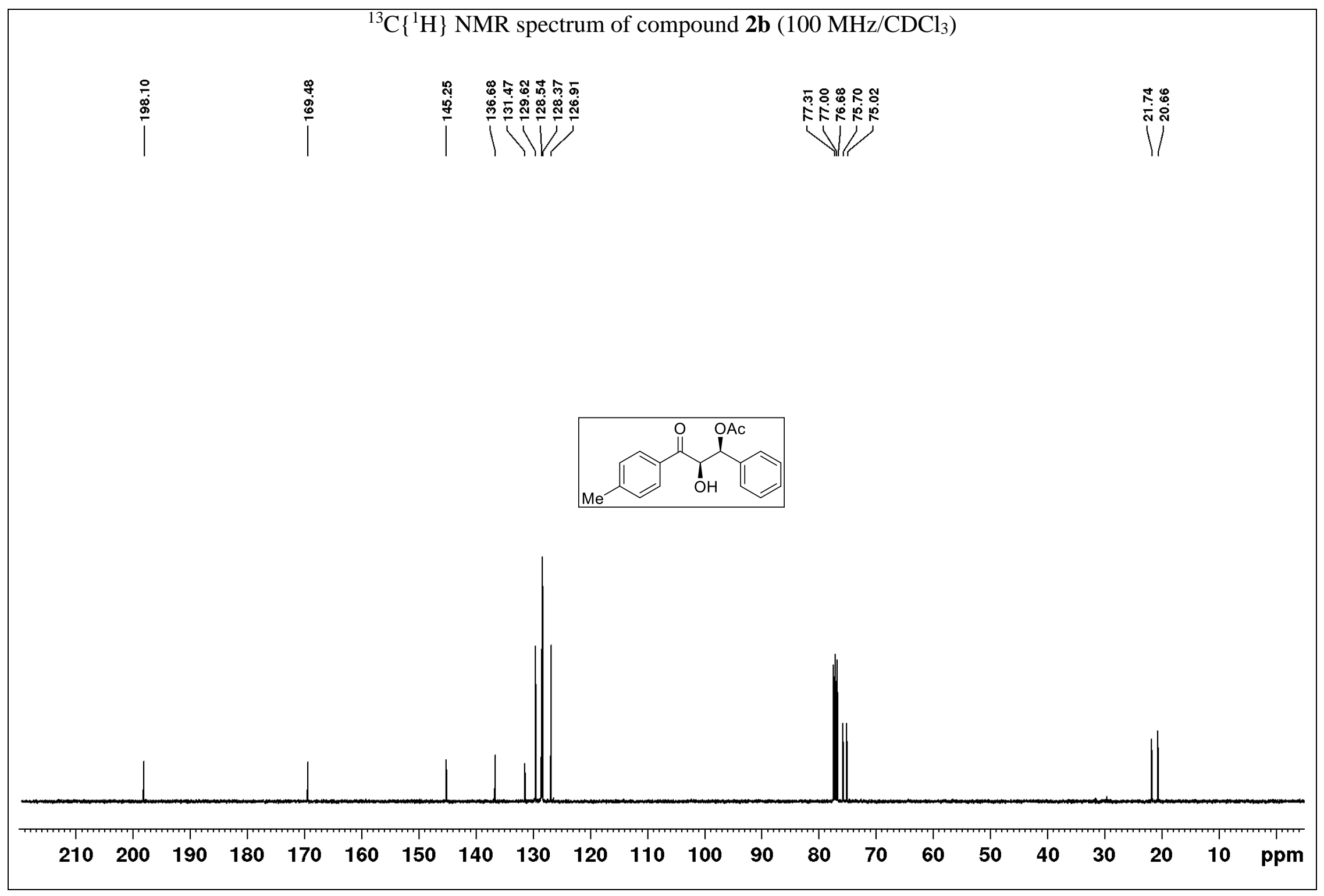

S16 


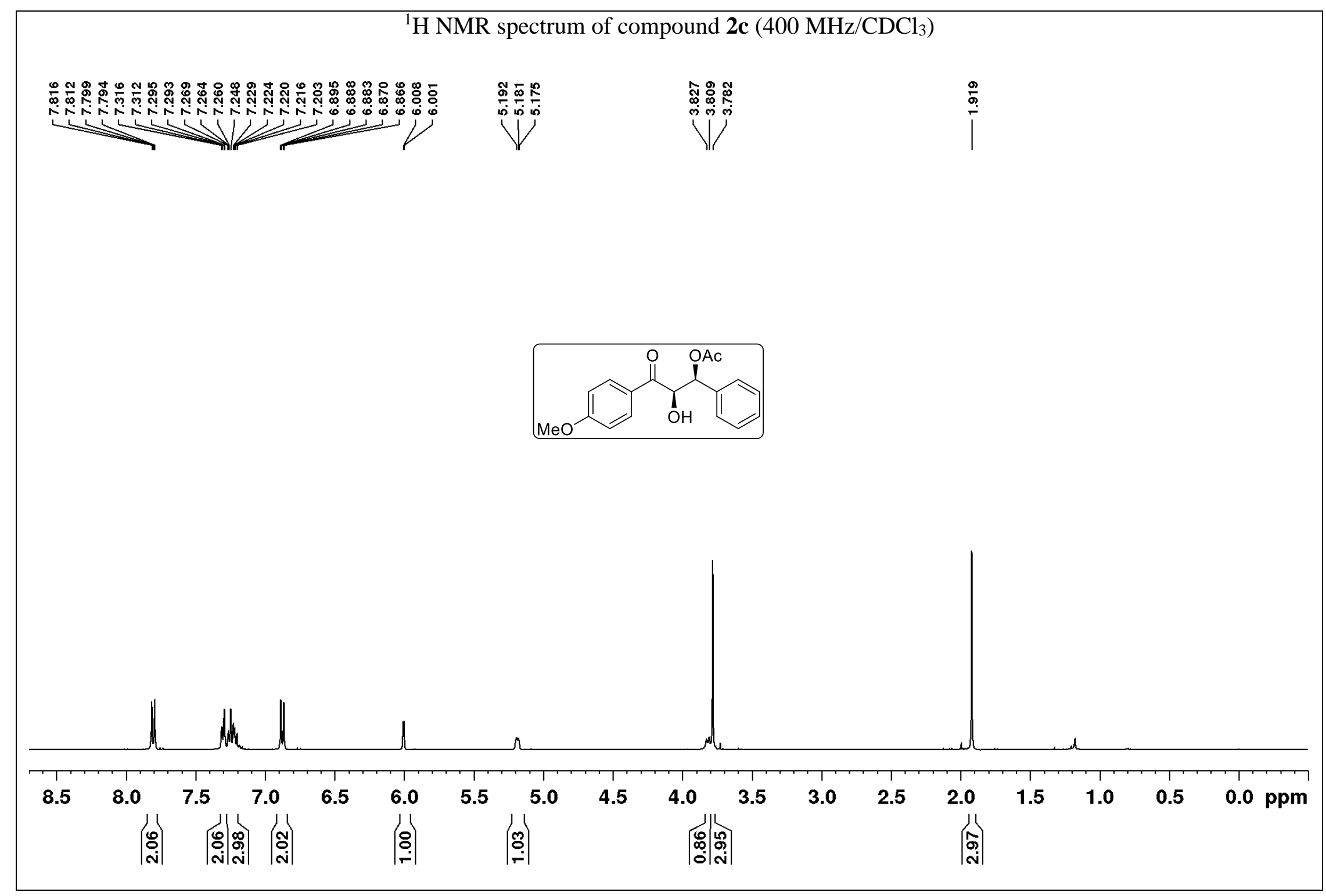




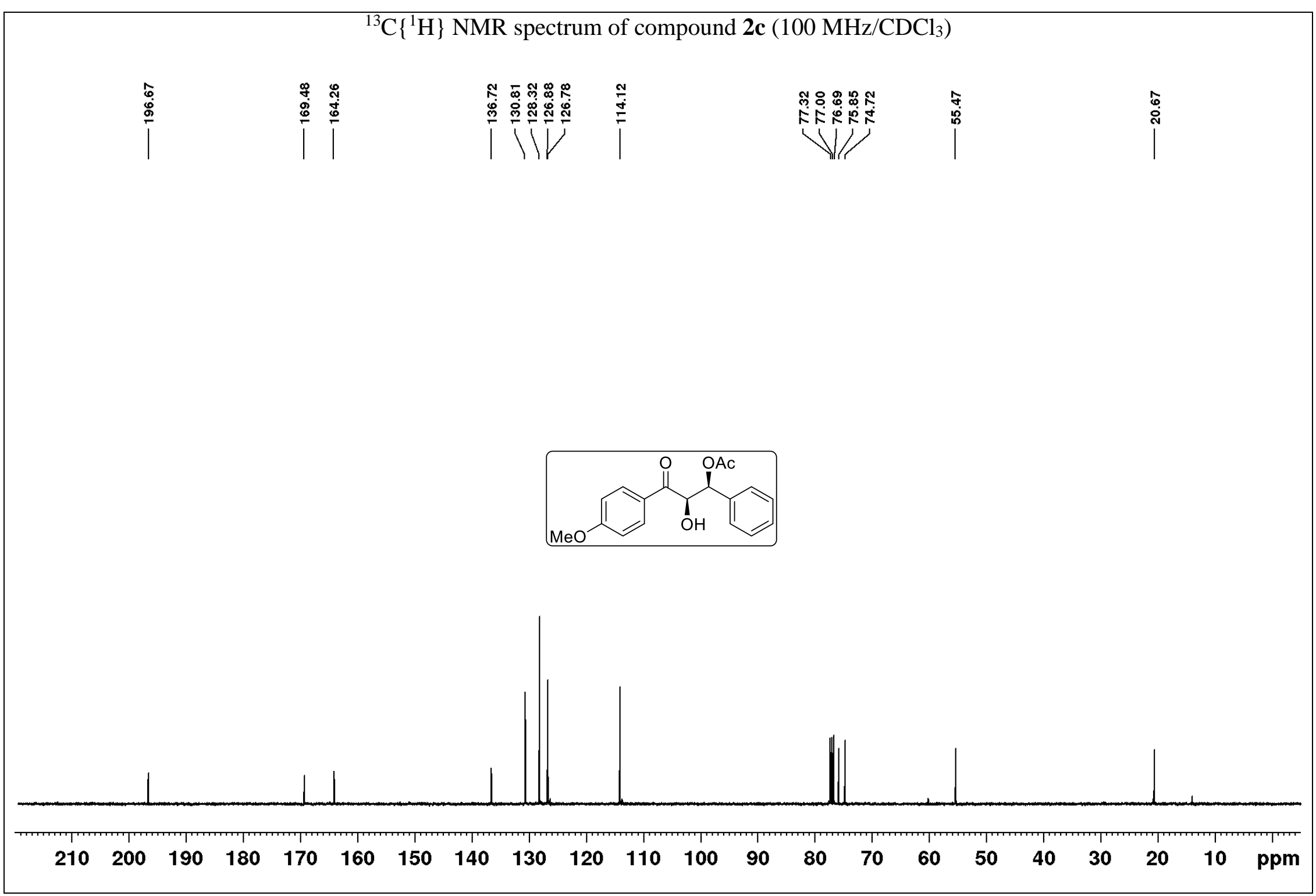




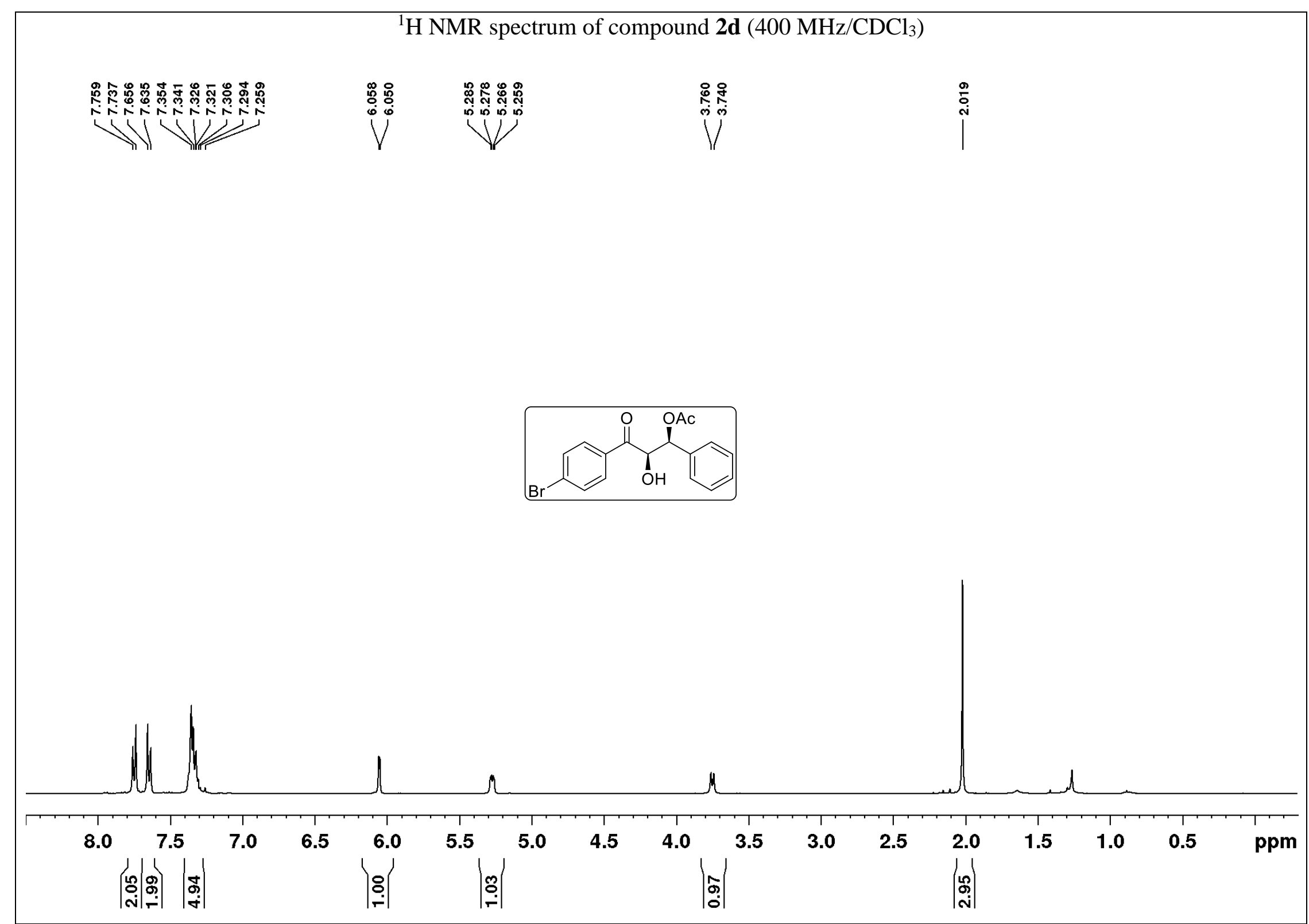




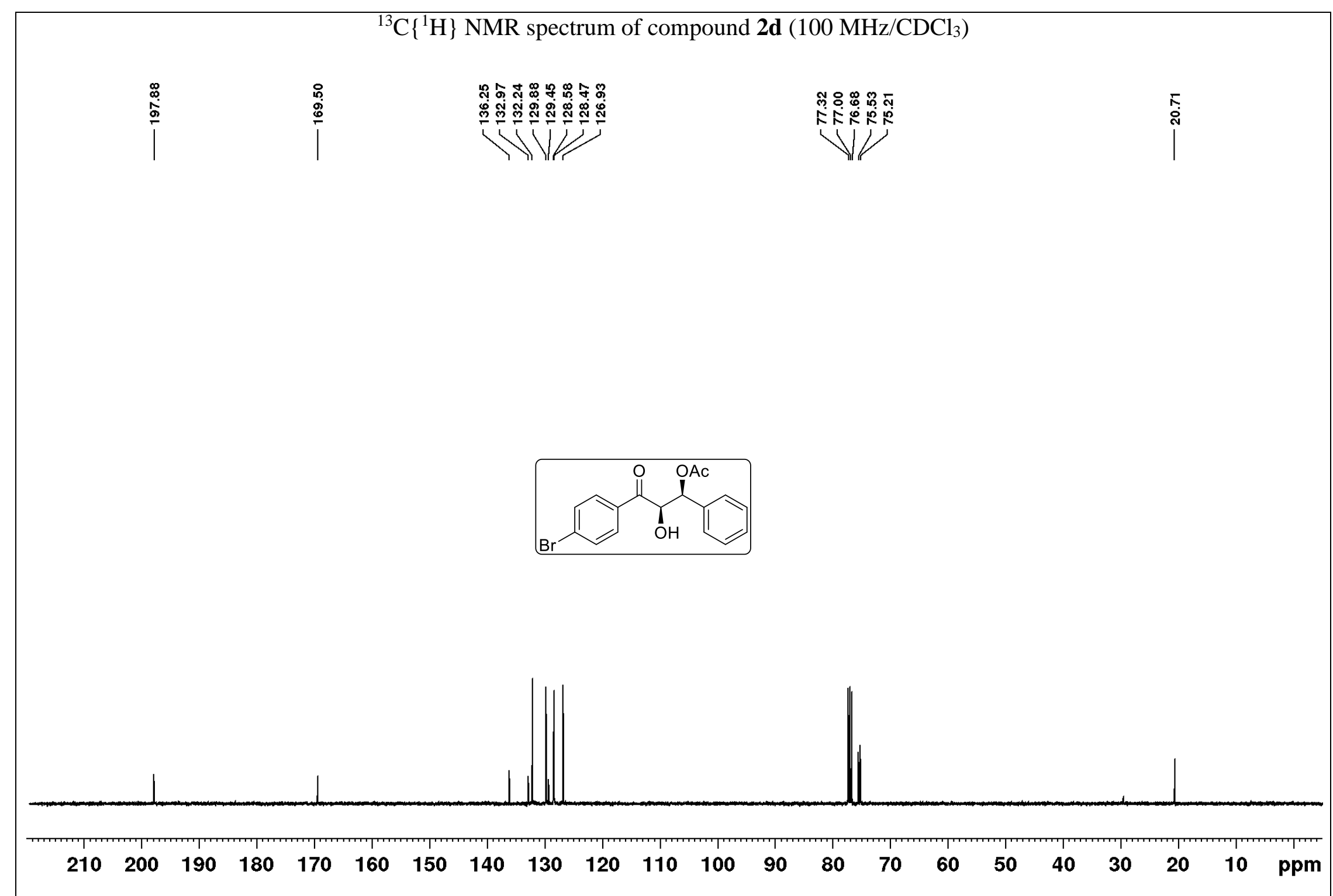




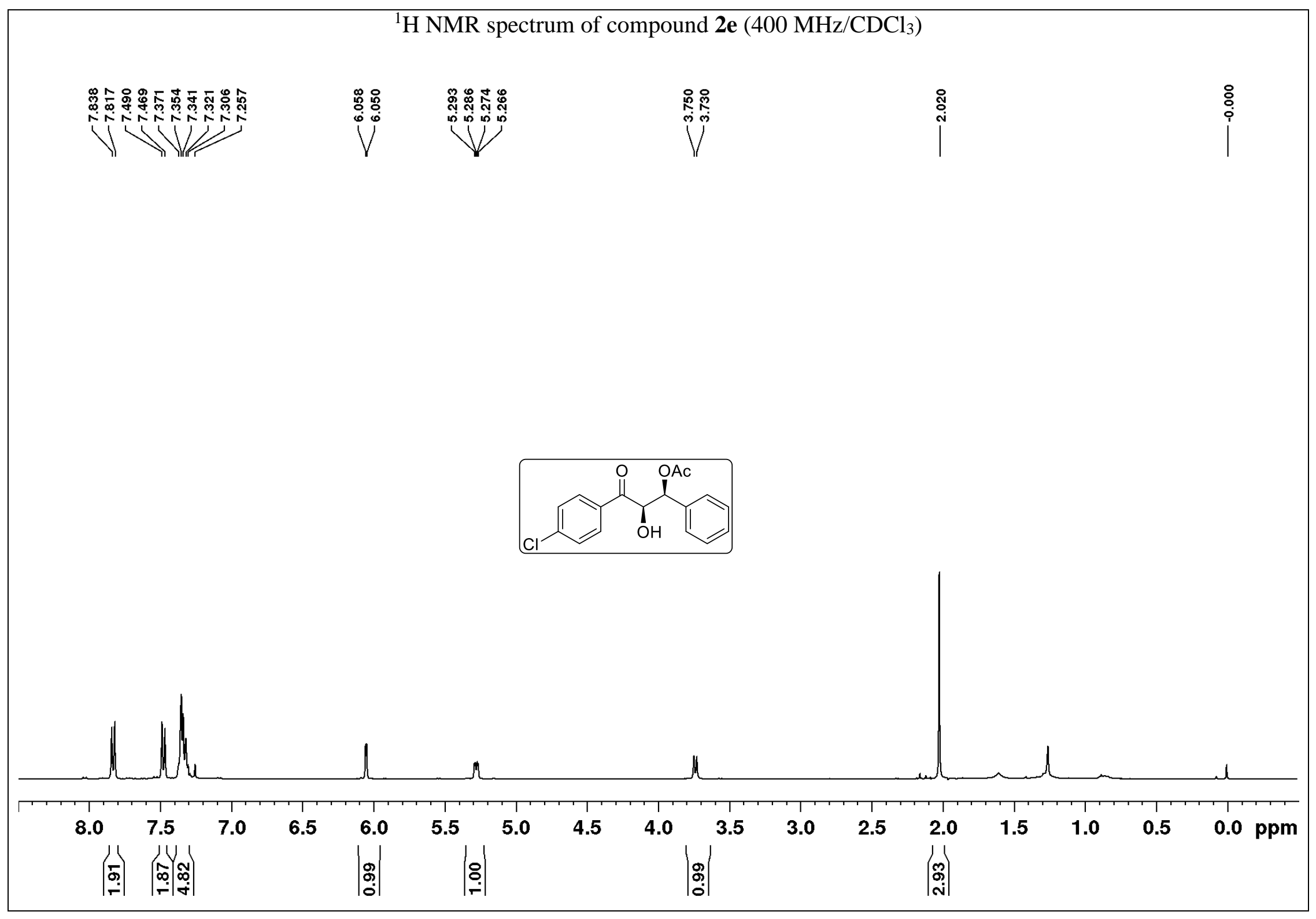

S21 


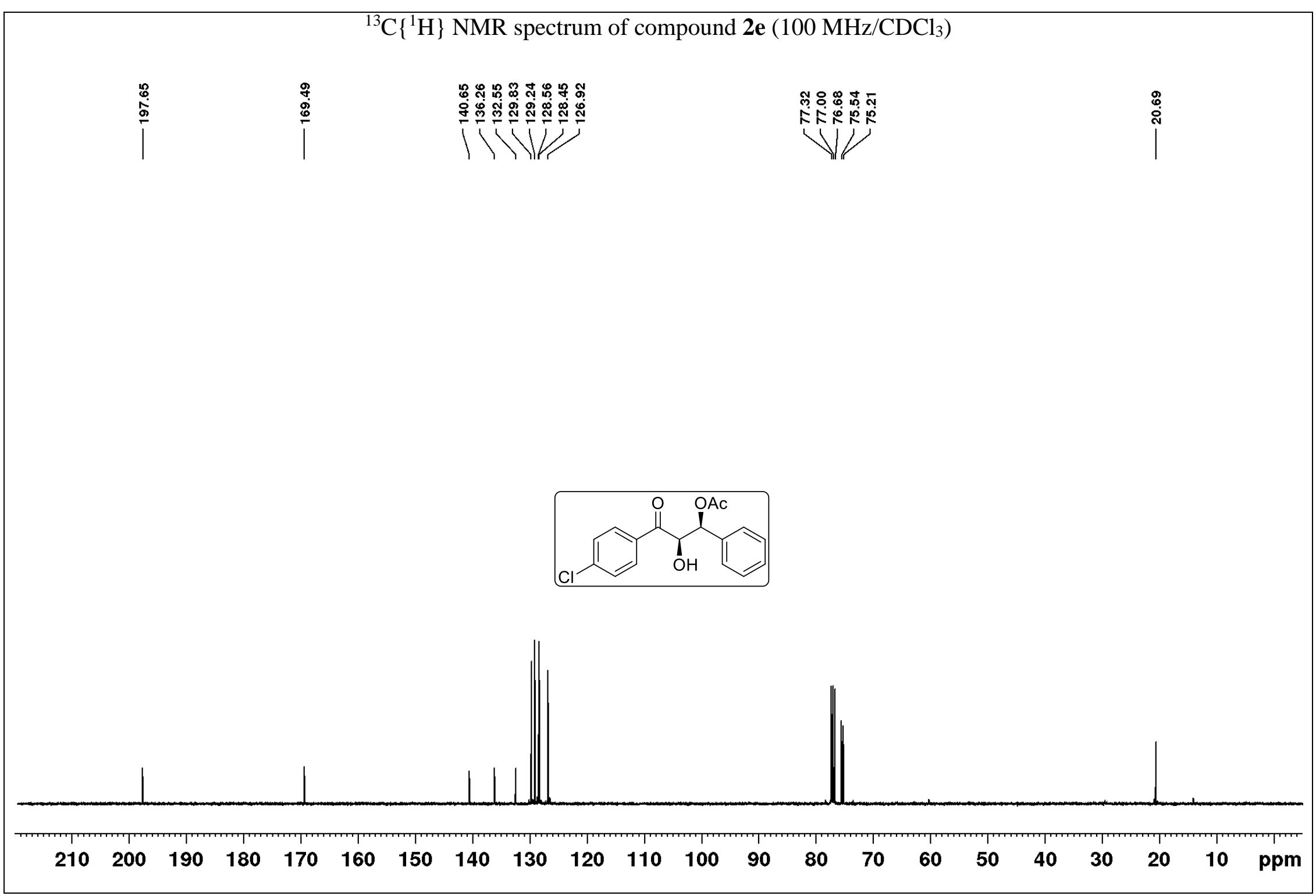




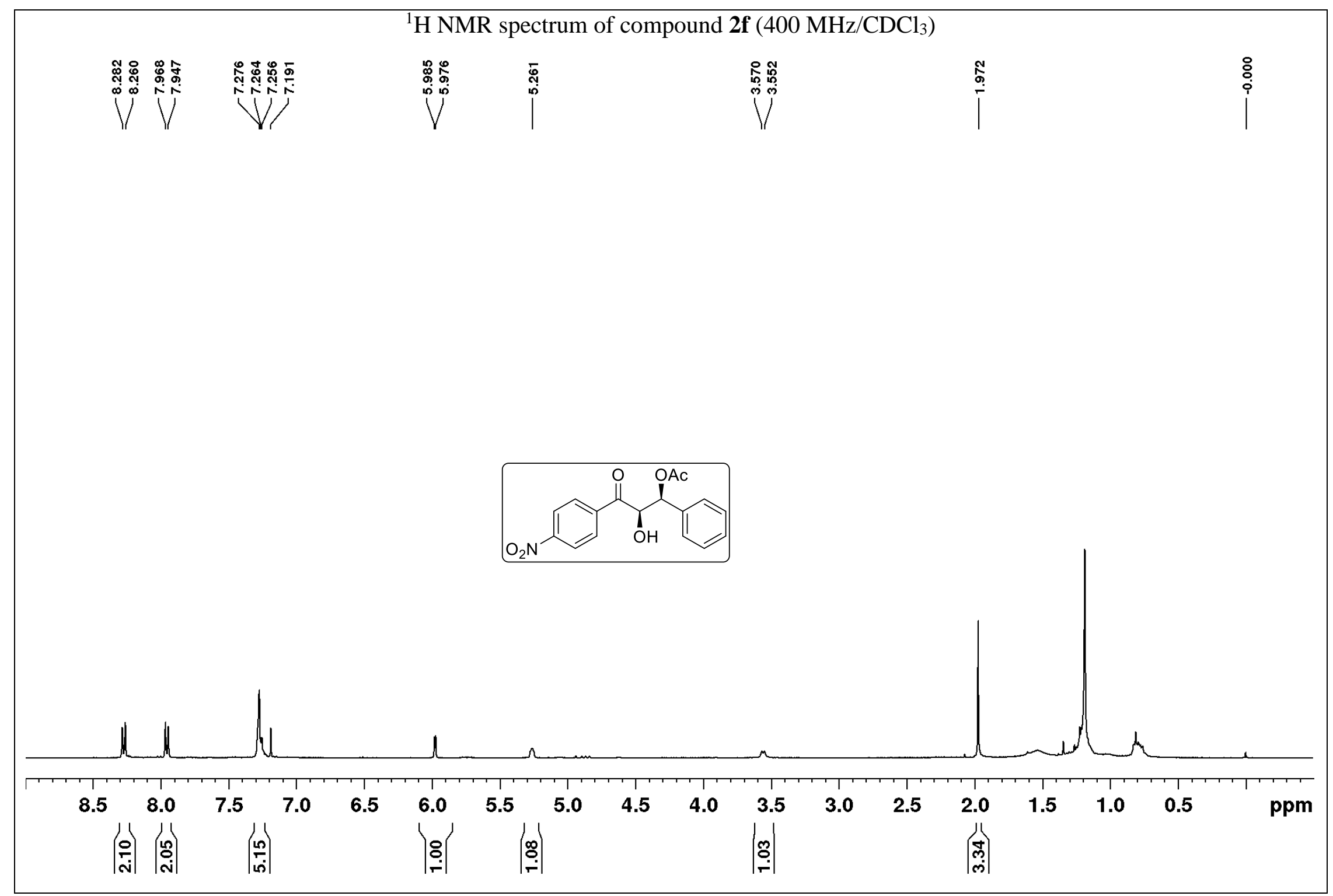




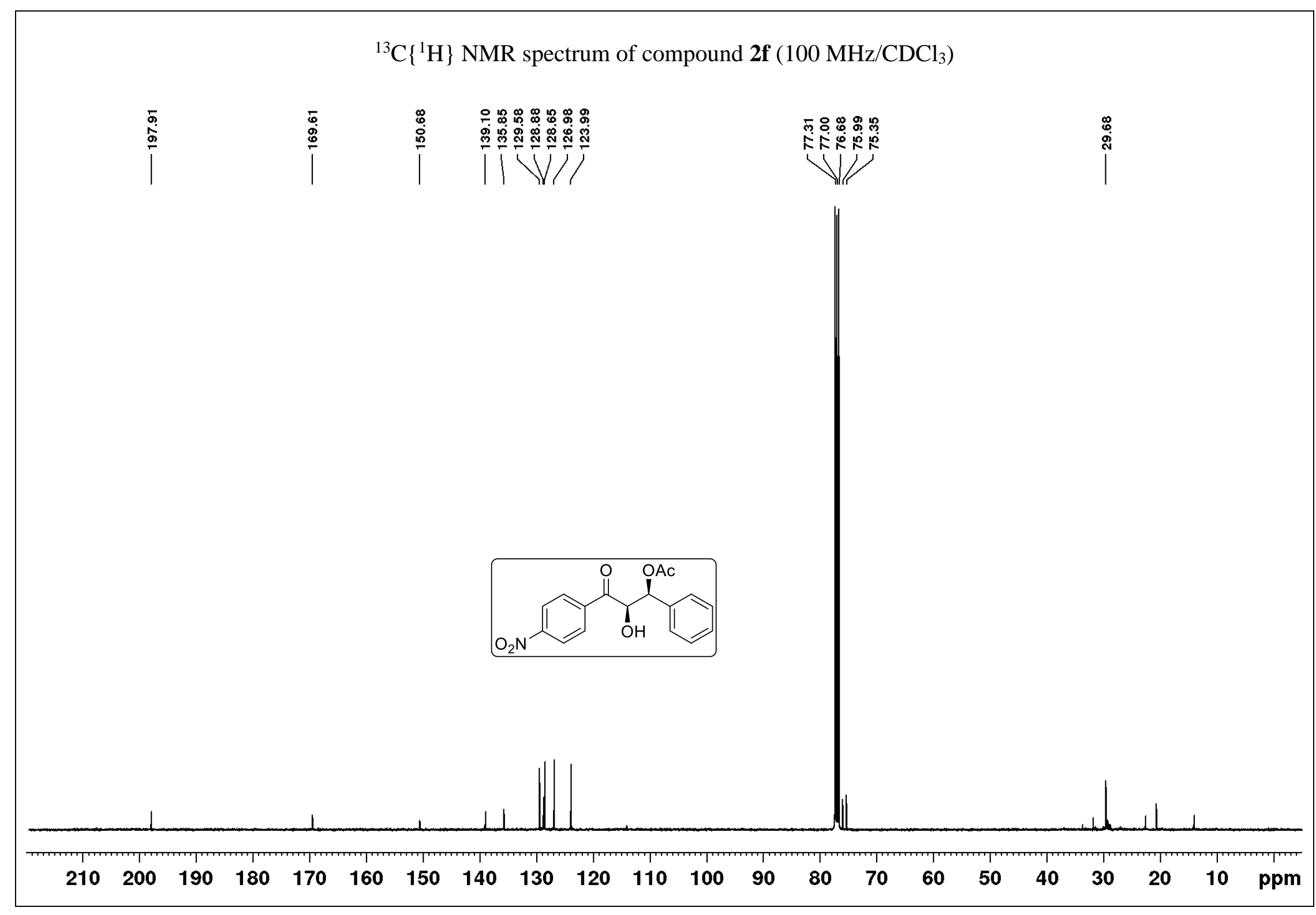




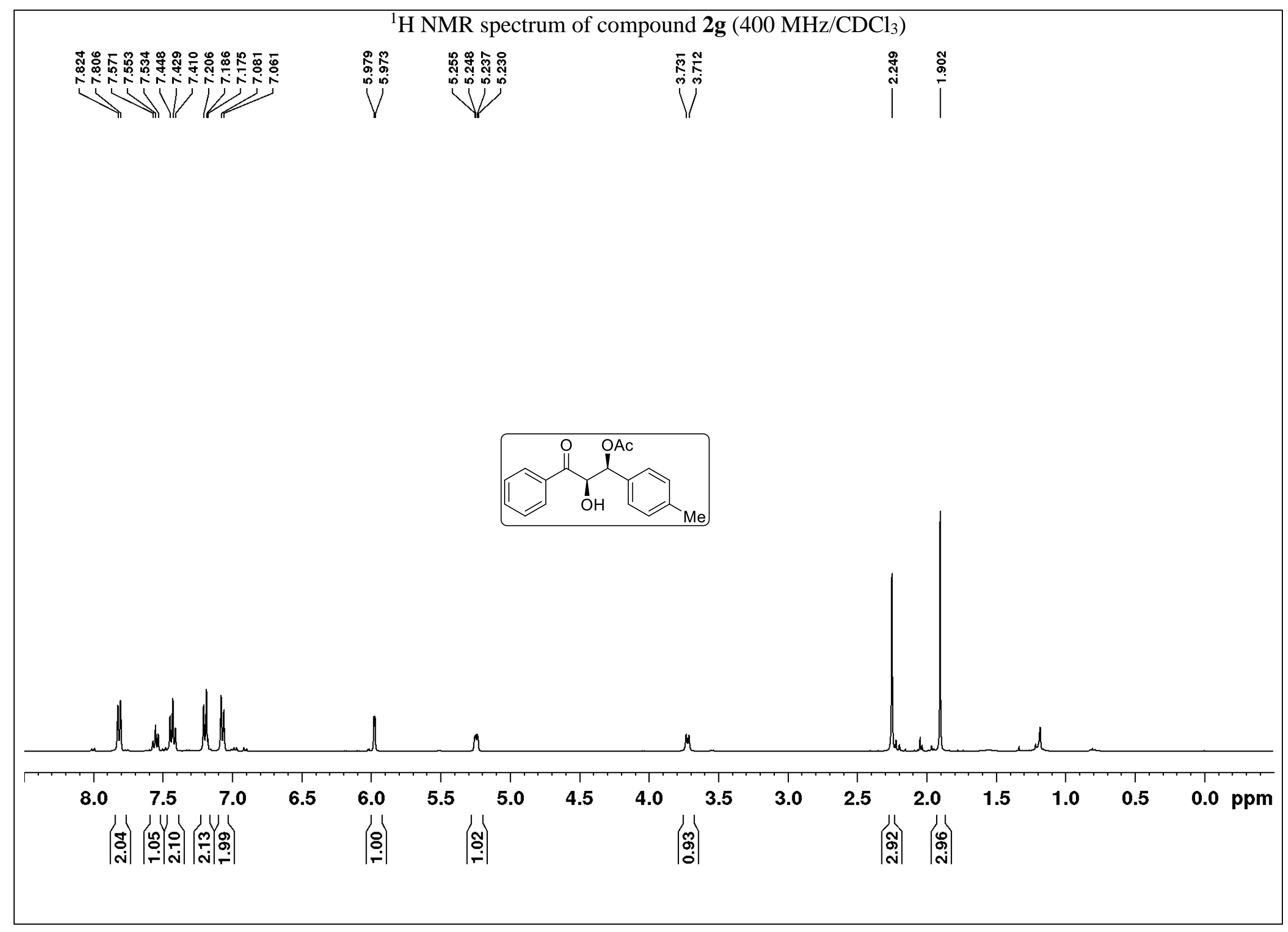




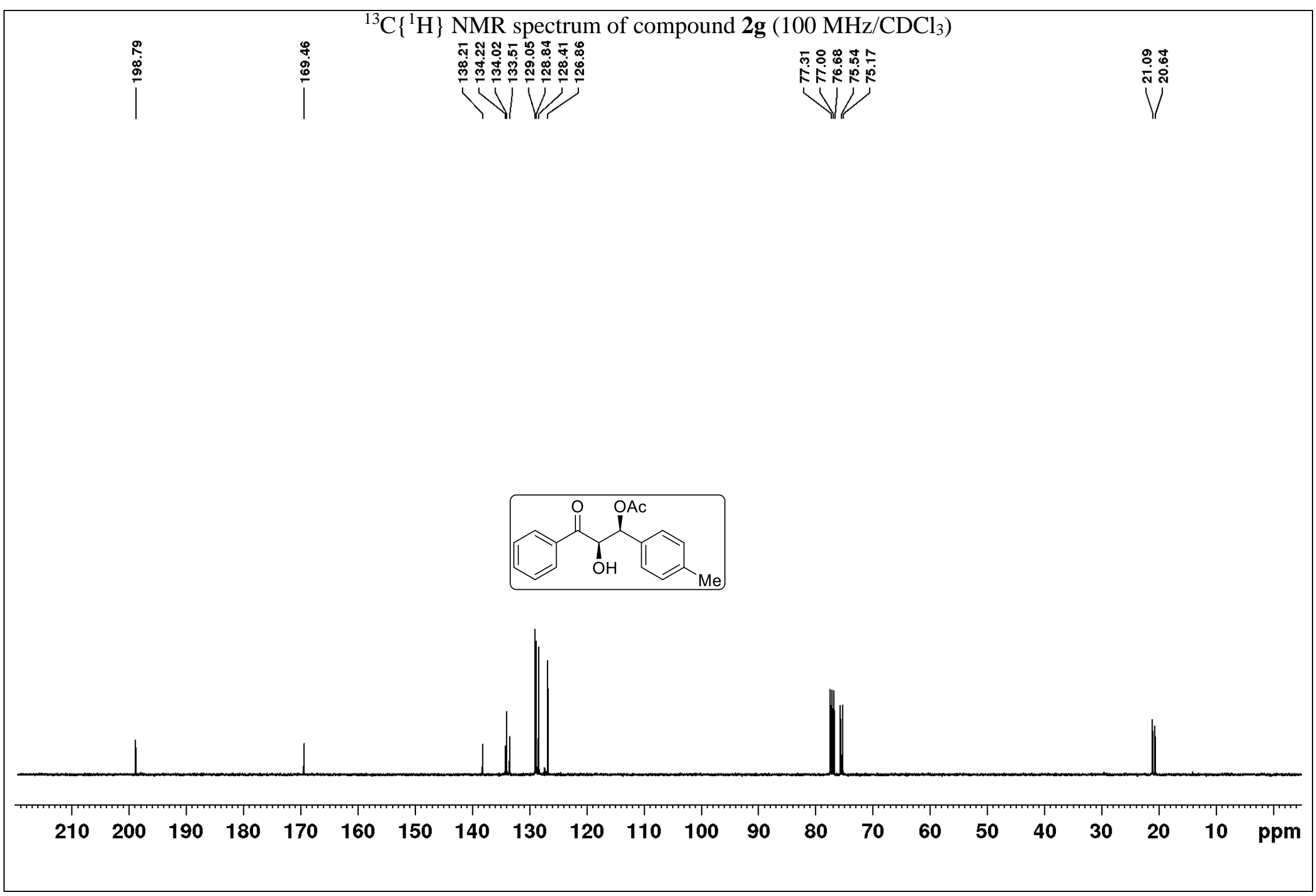




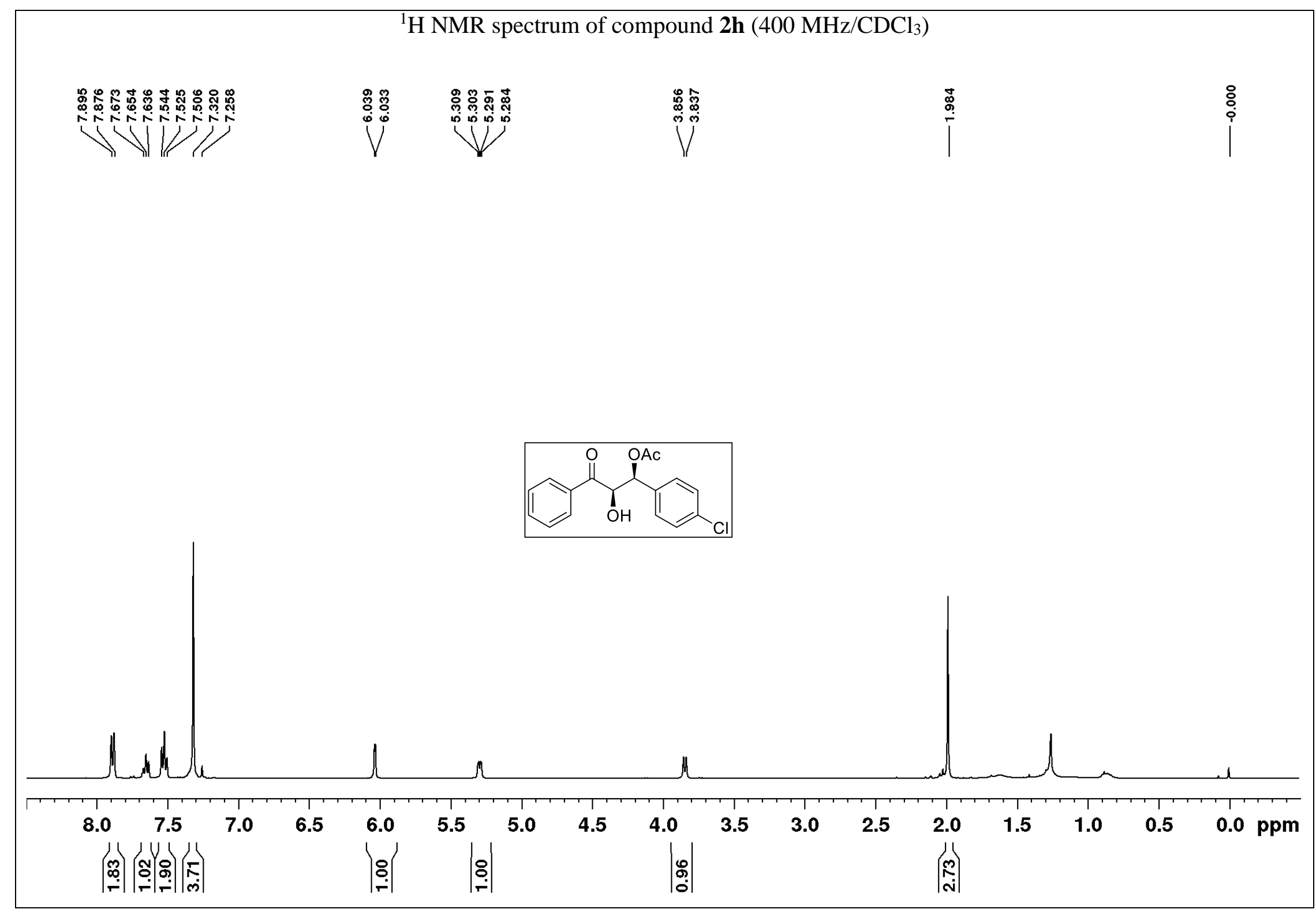




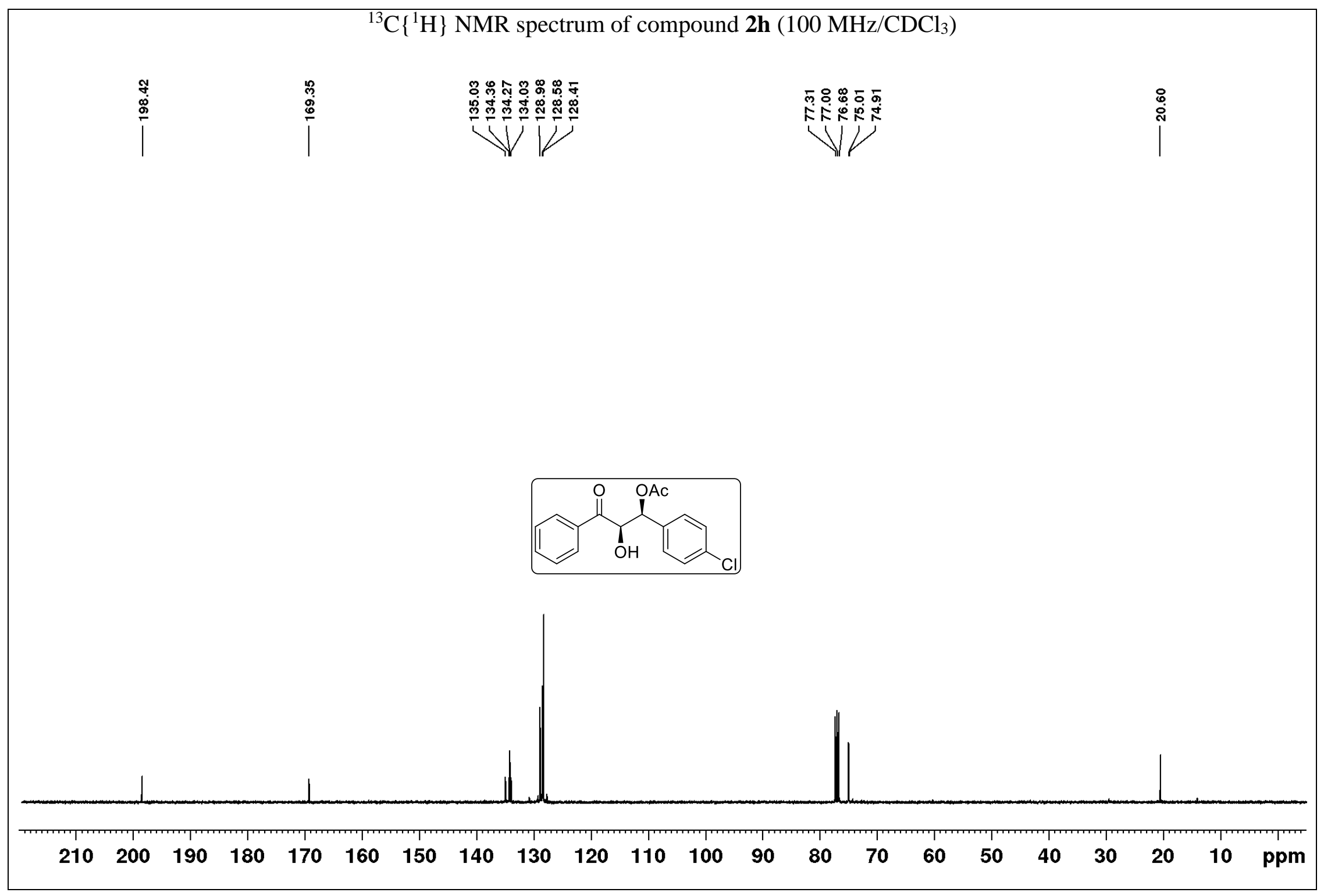

S28 


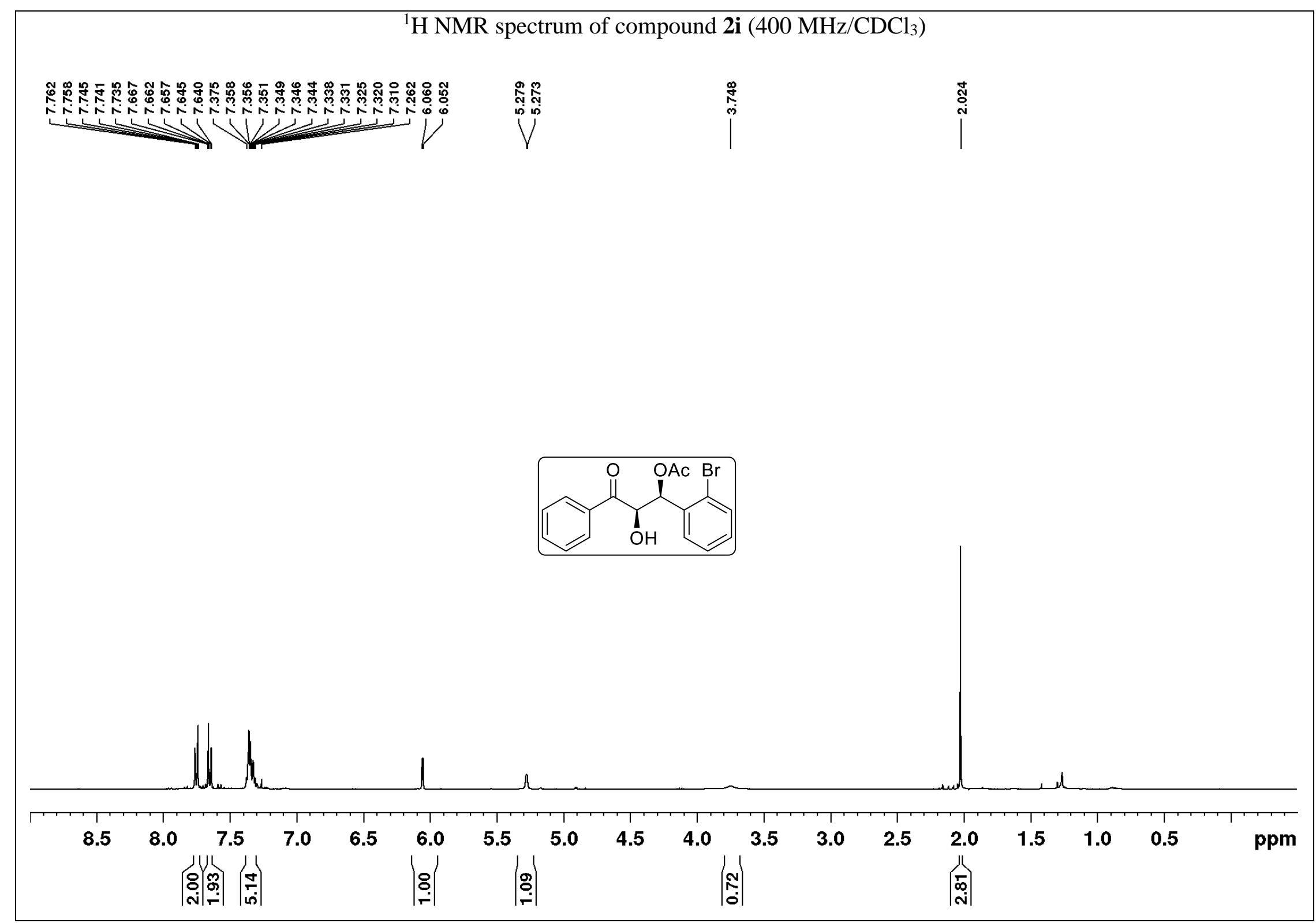




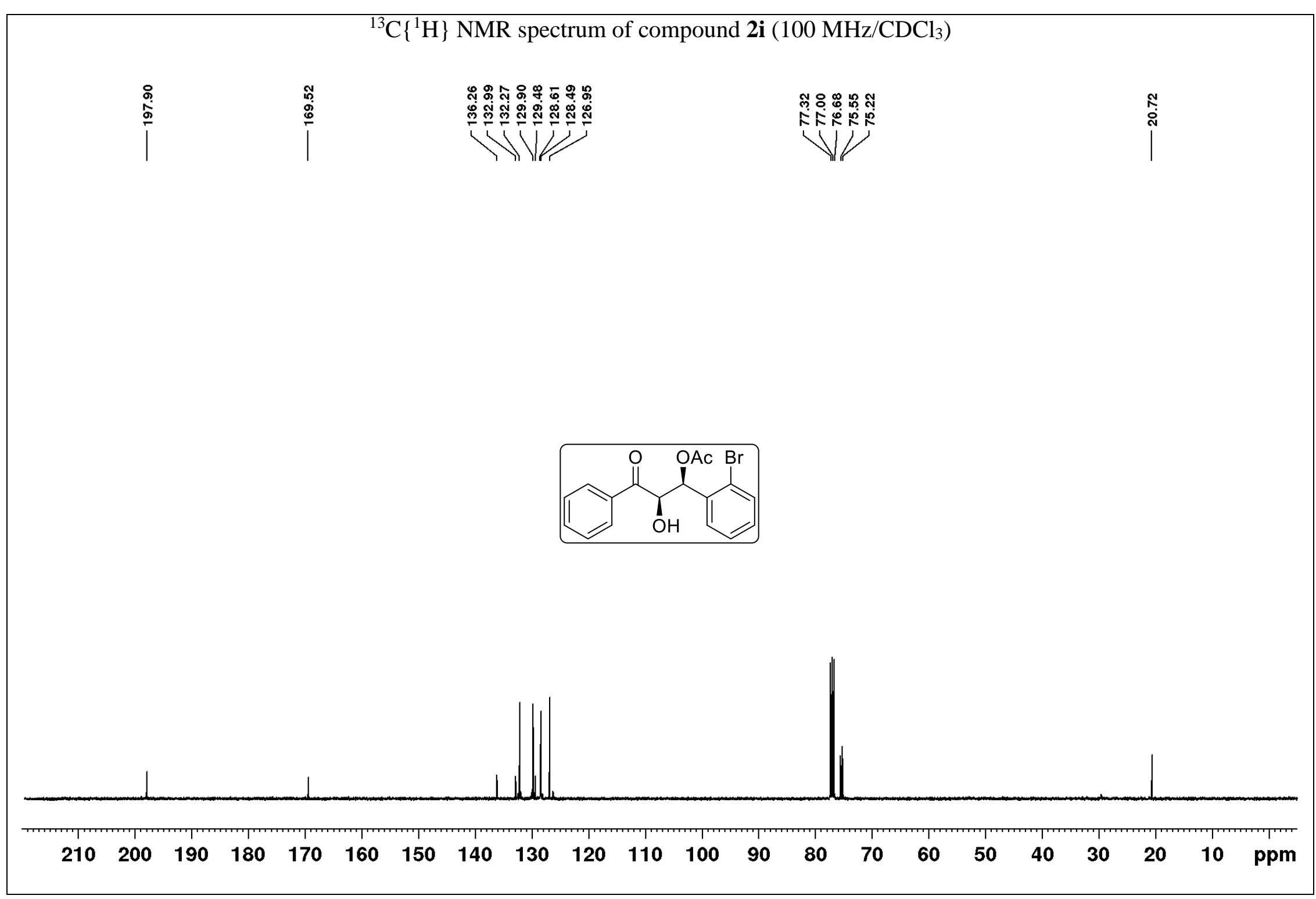




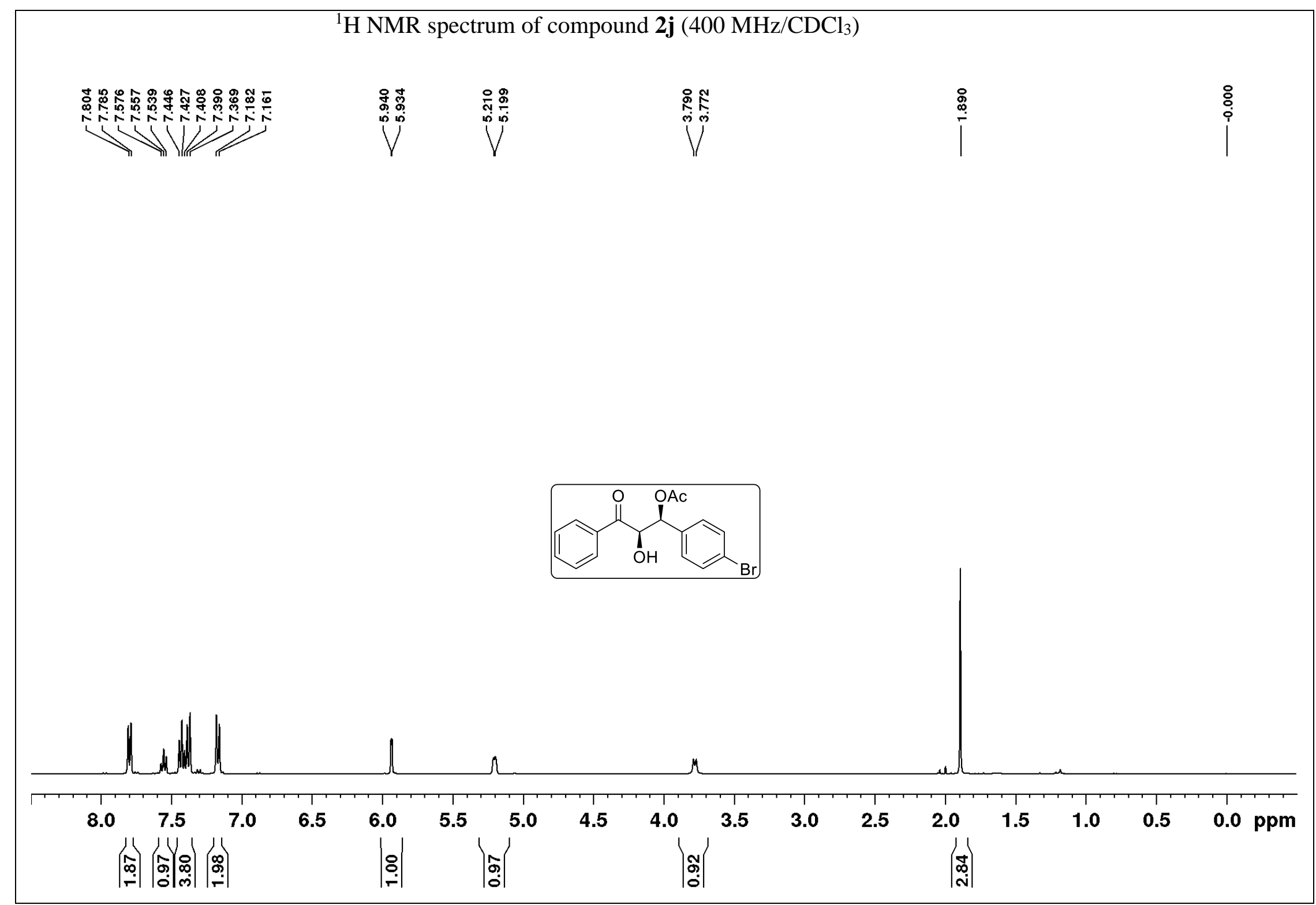




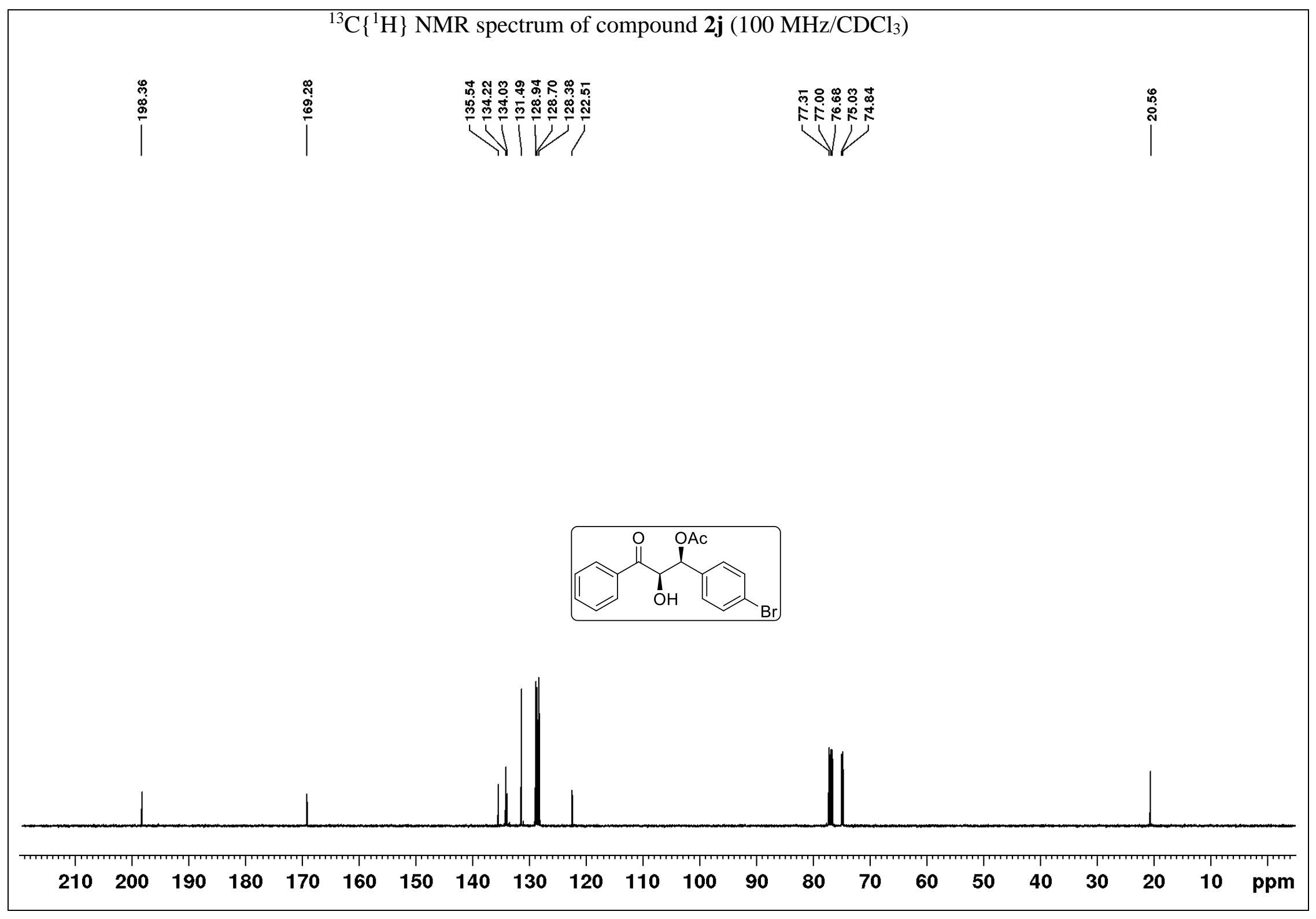

S32 


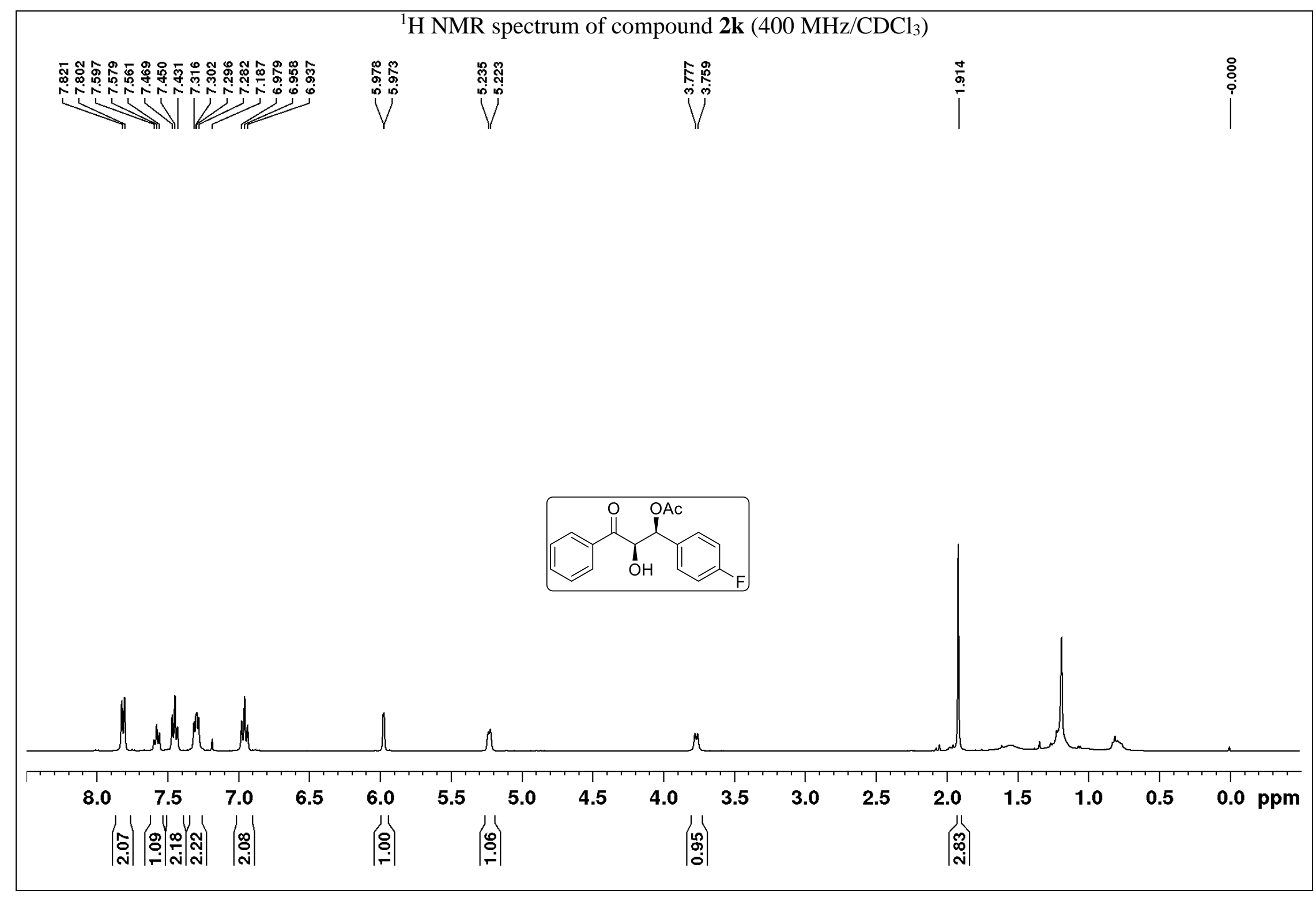




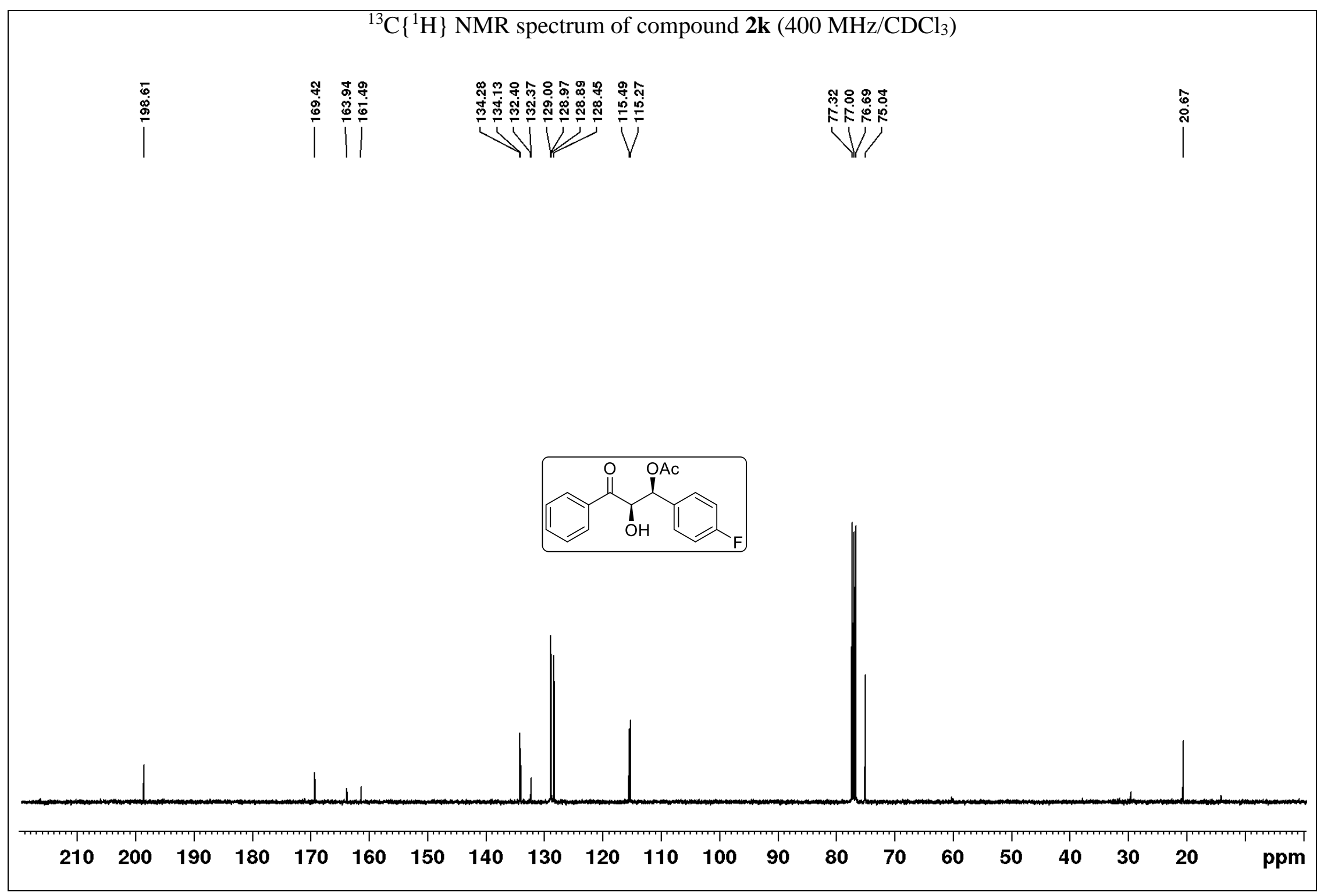

S34 


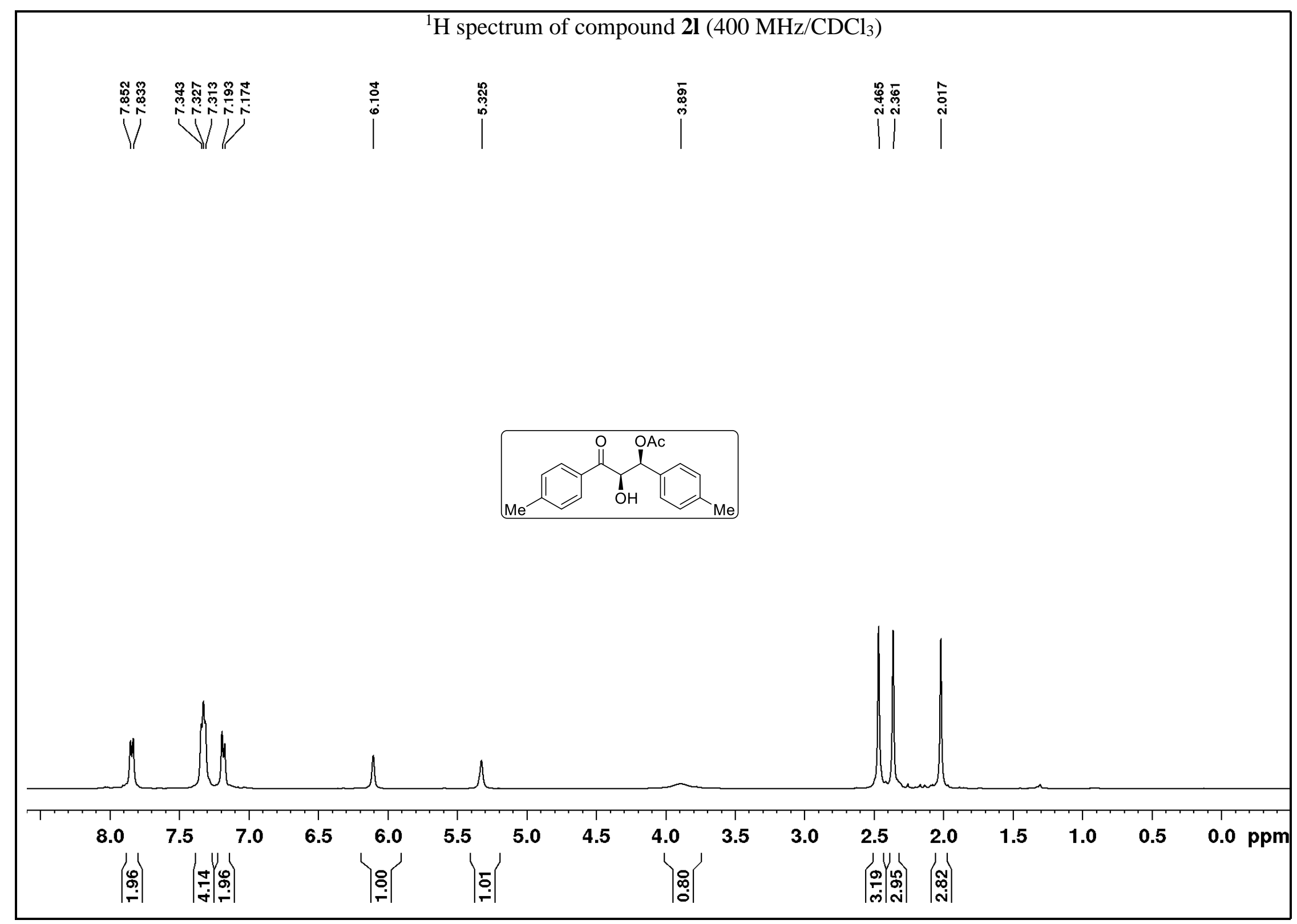




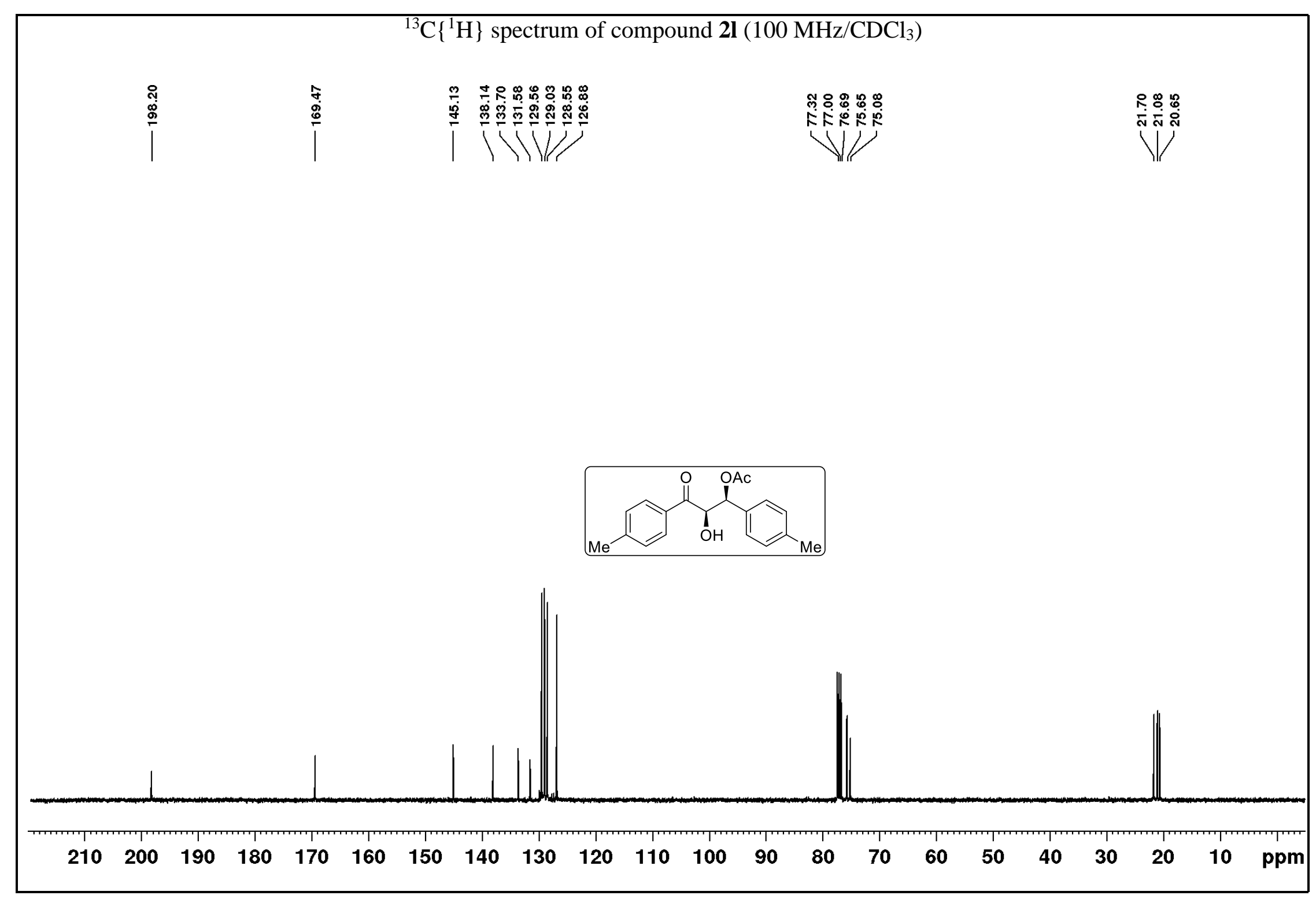




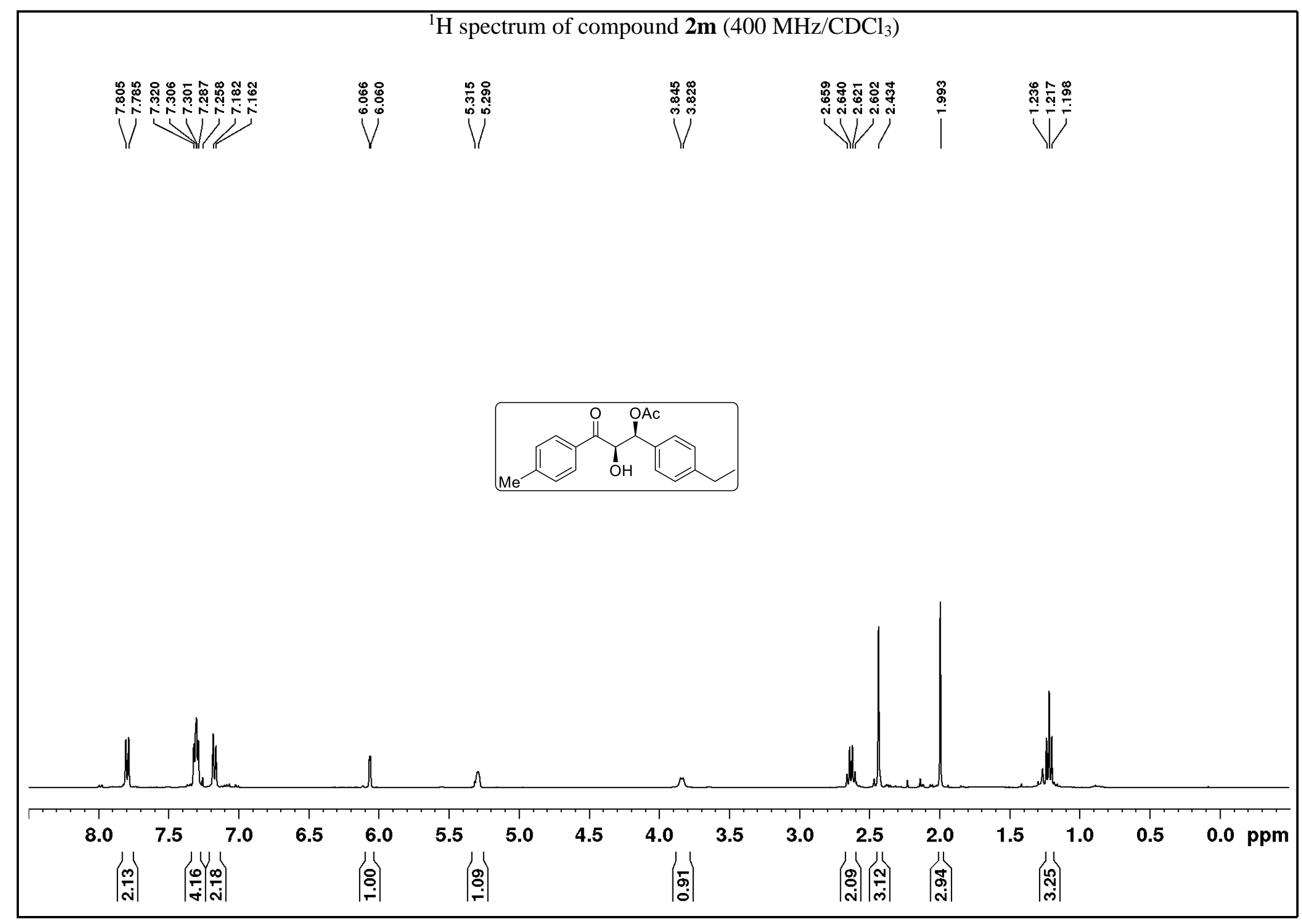




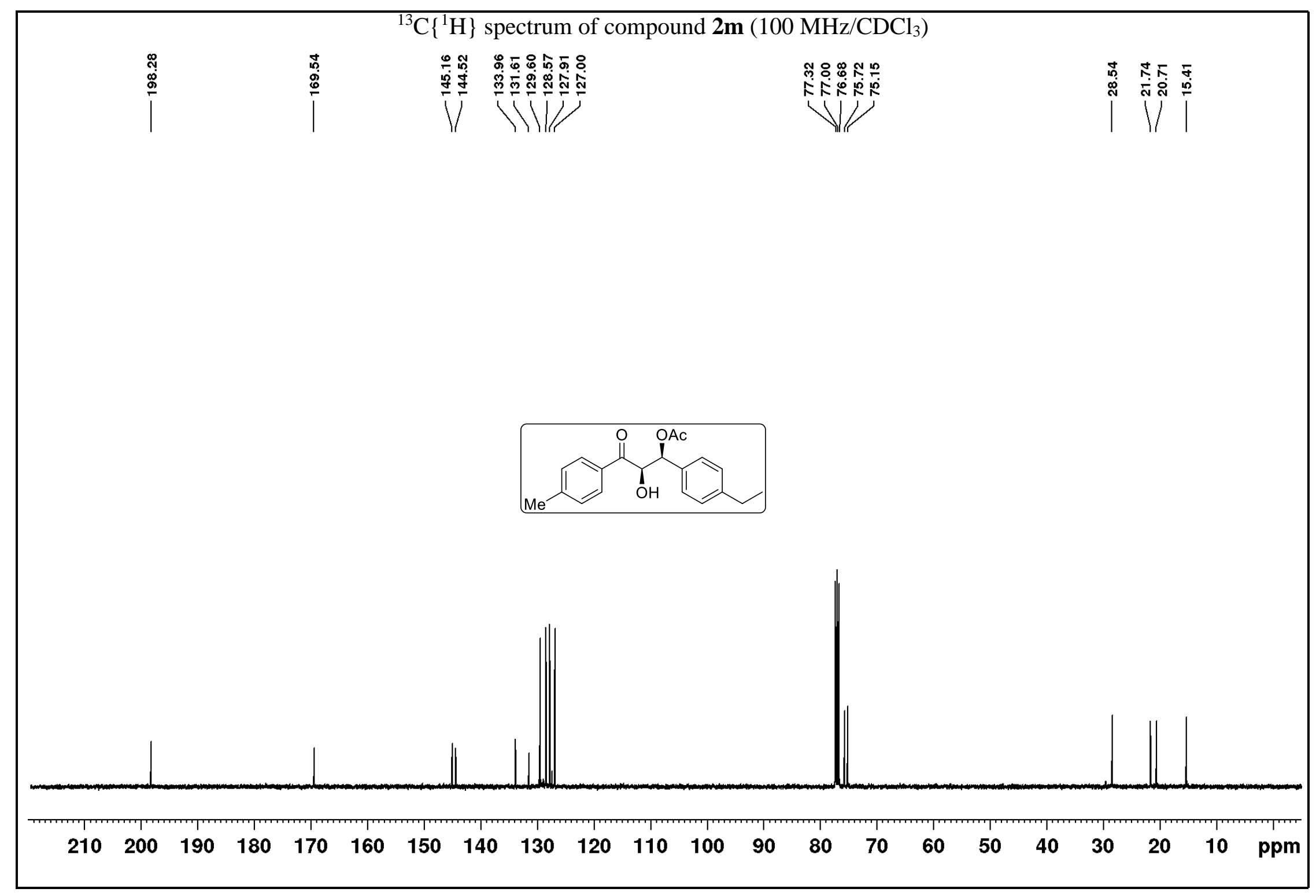




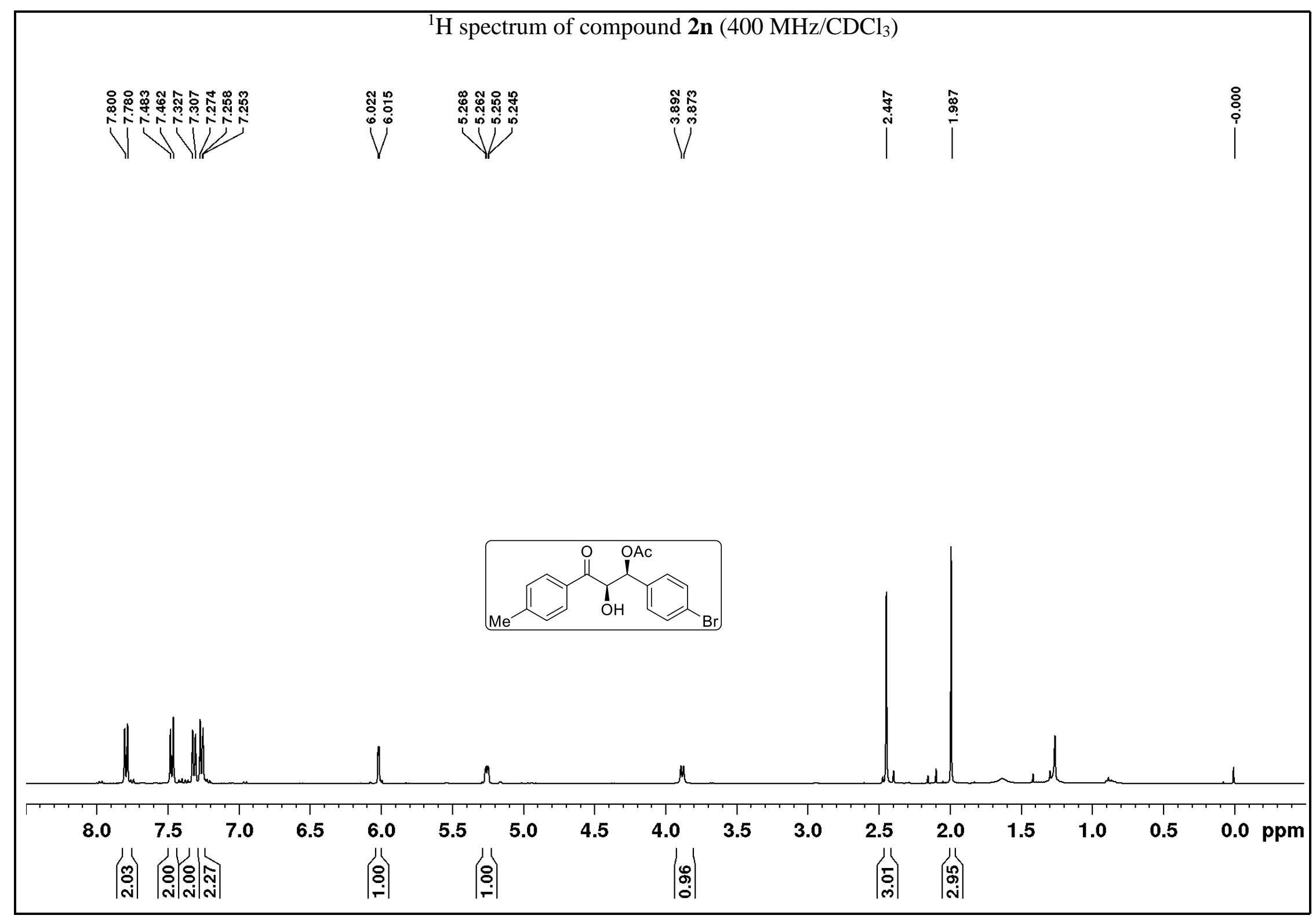




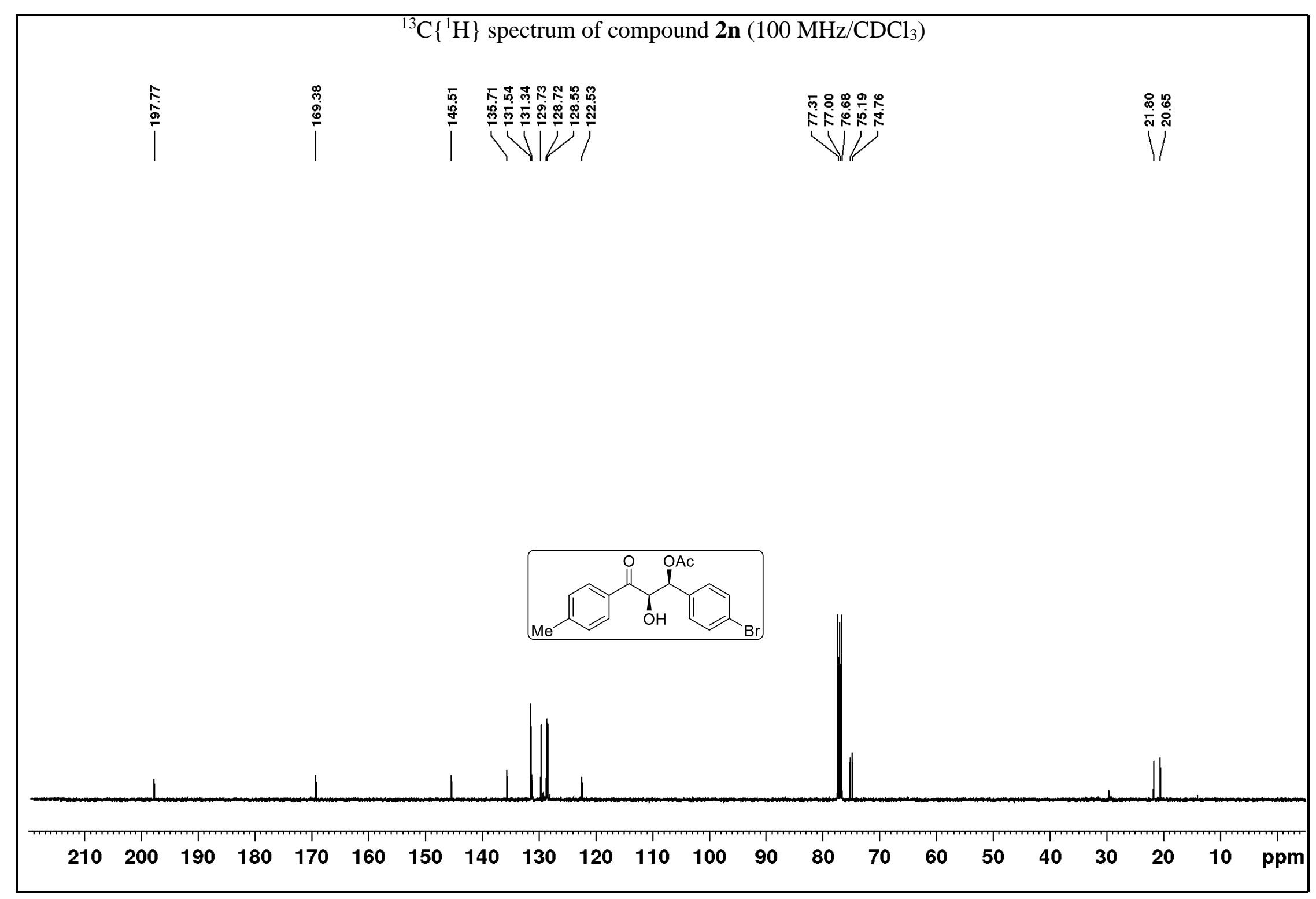




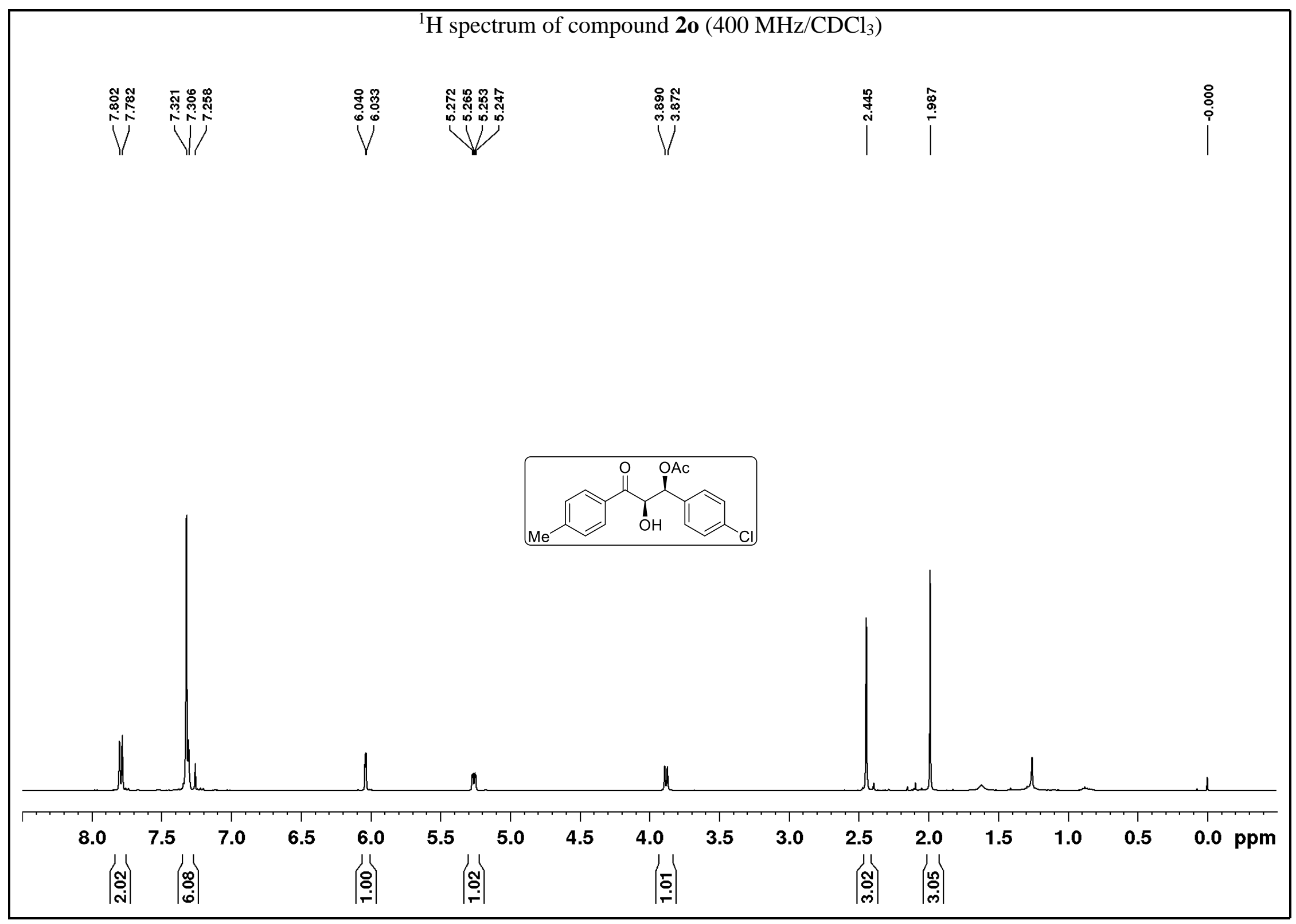

S41 


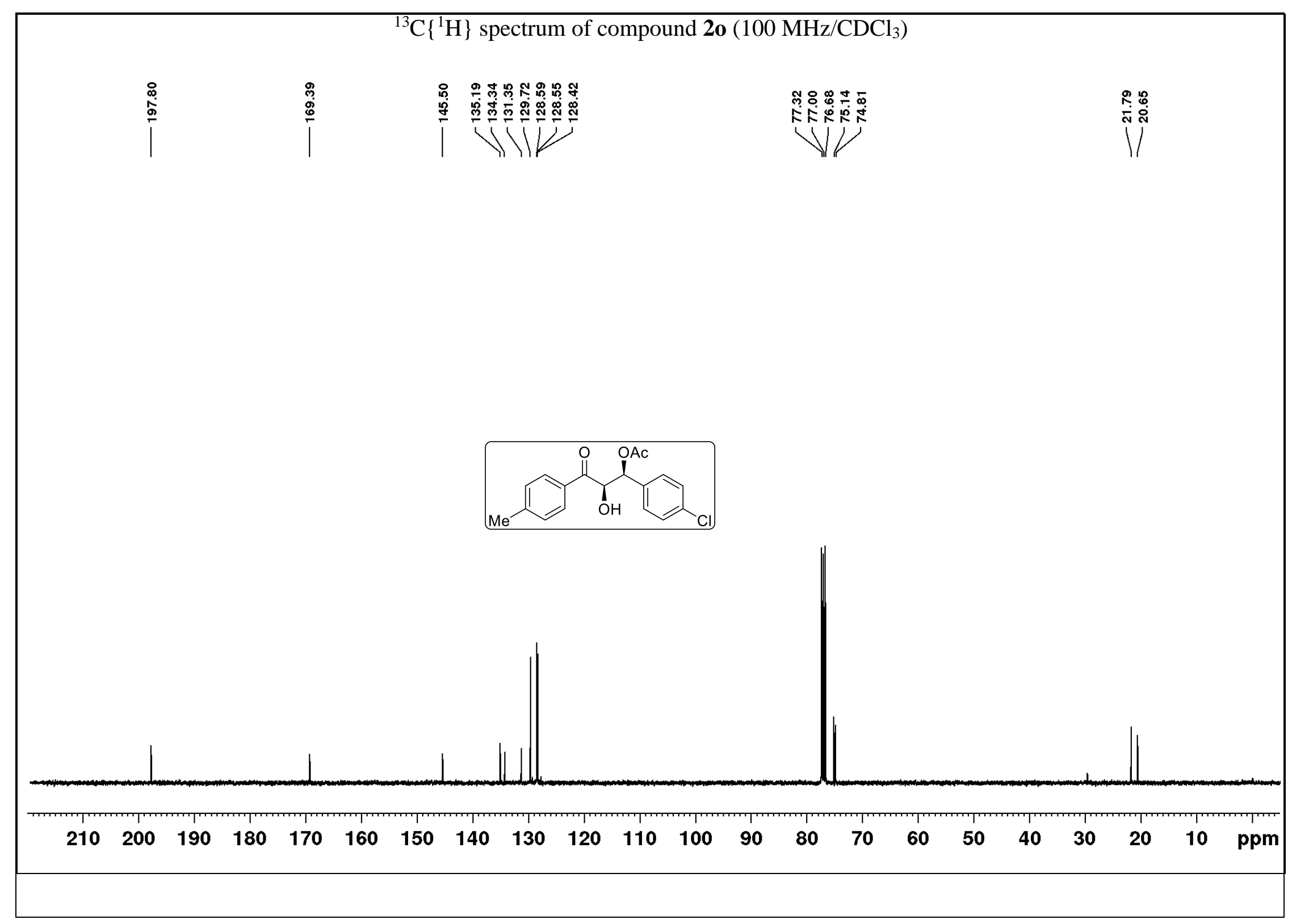




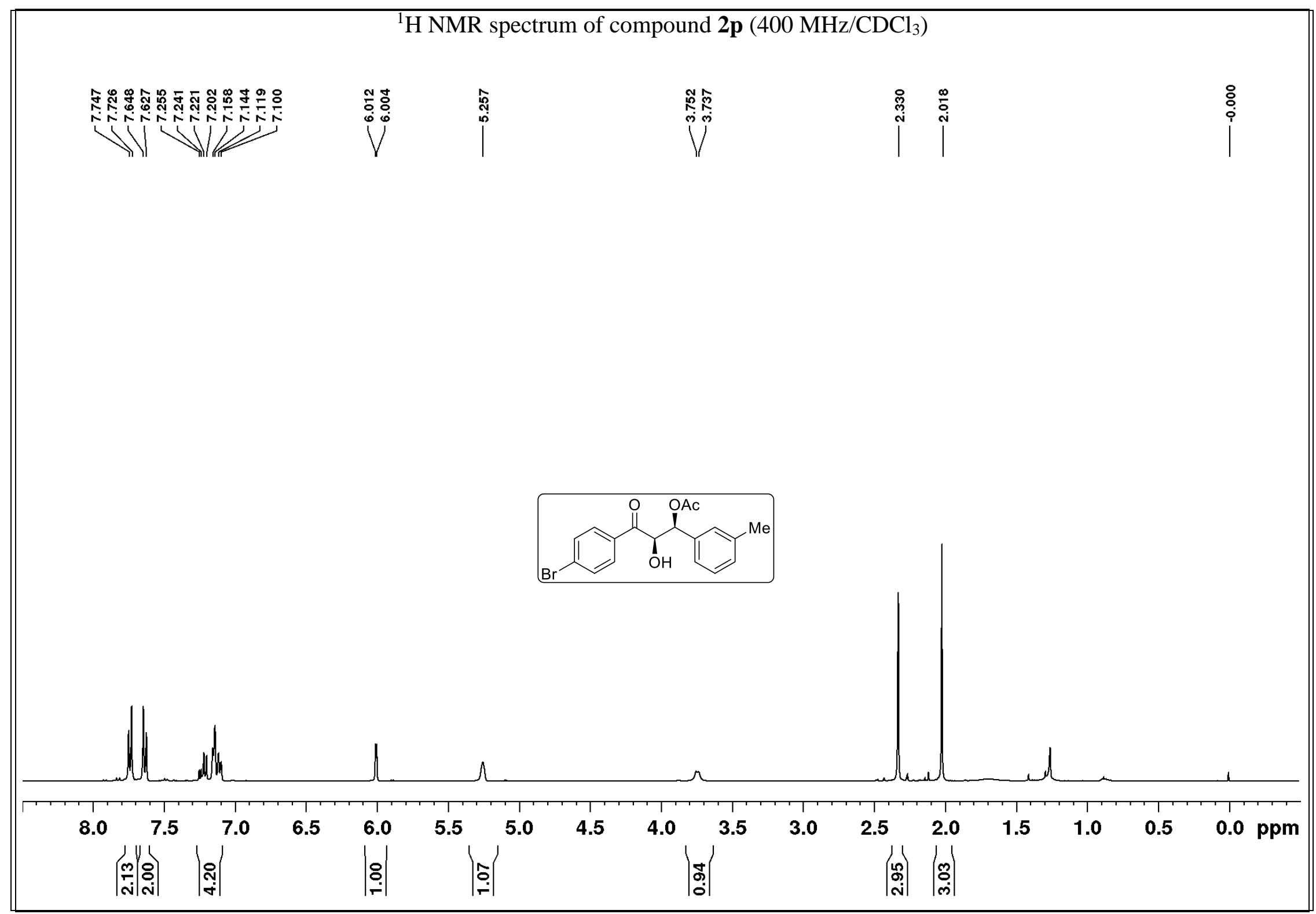




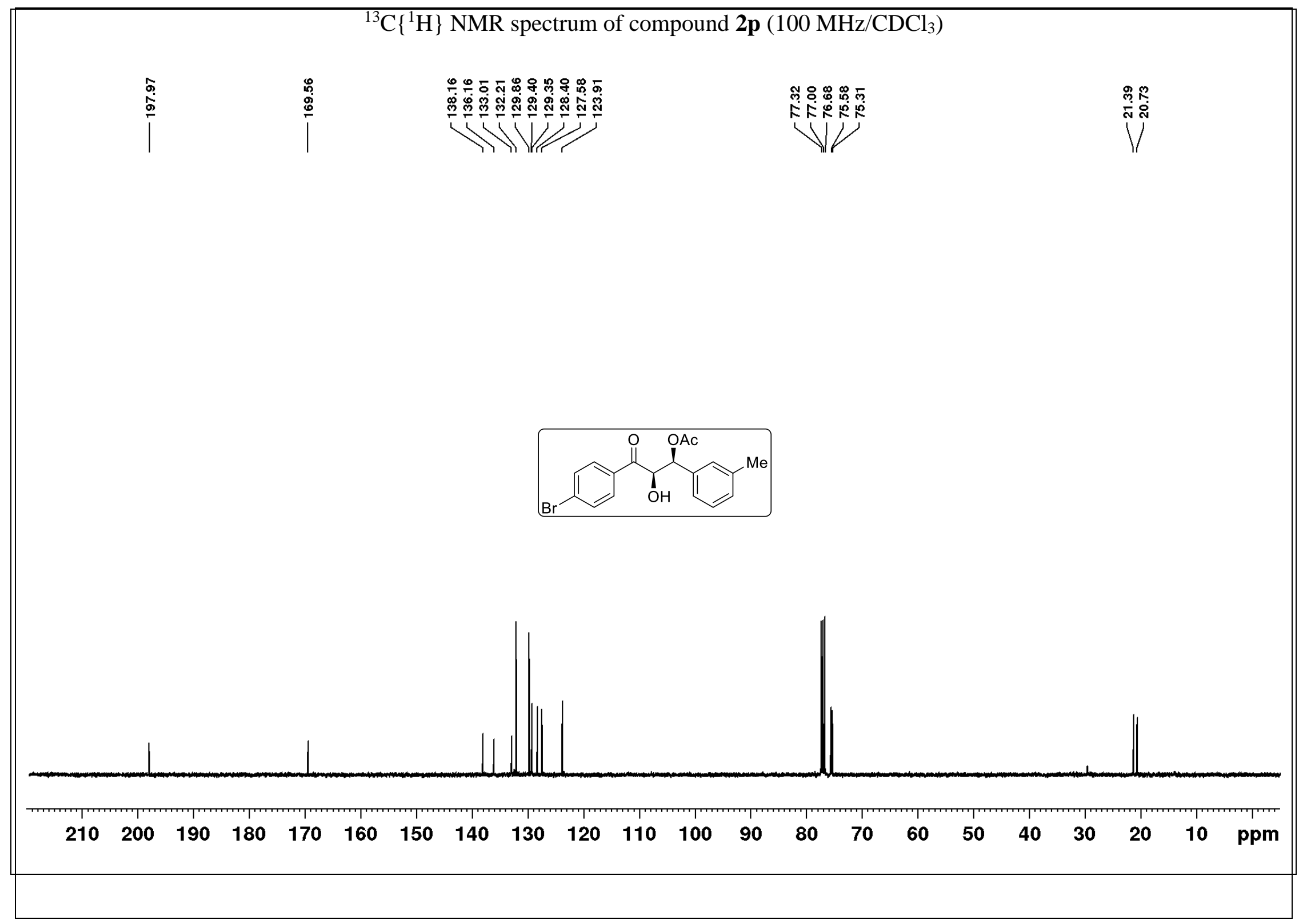




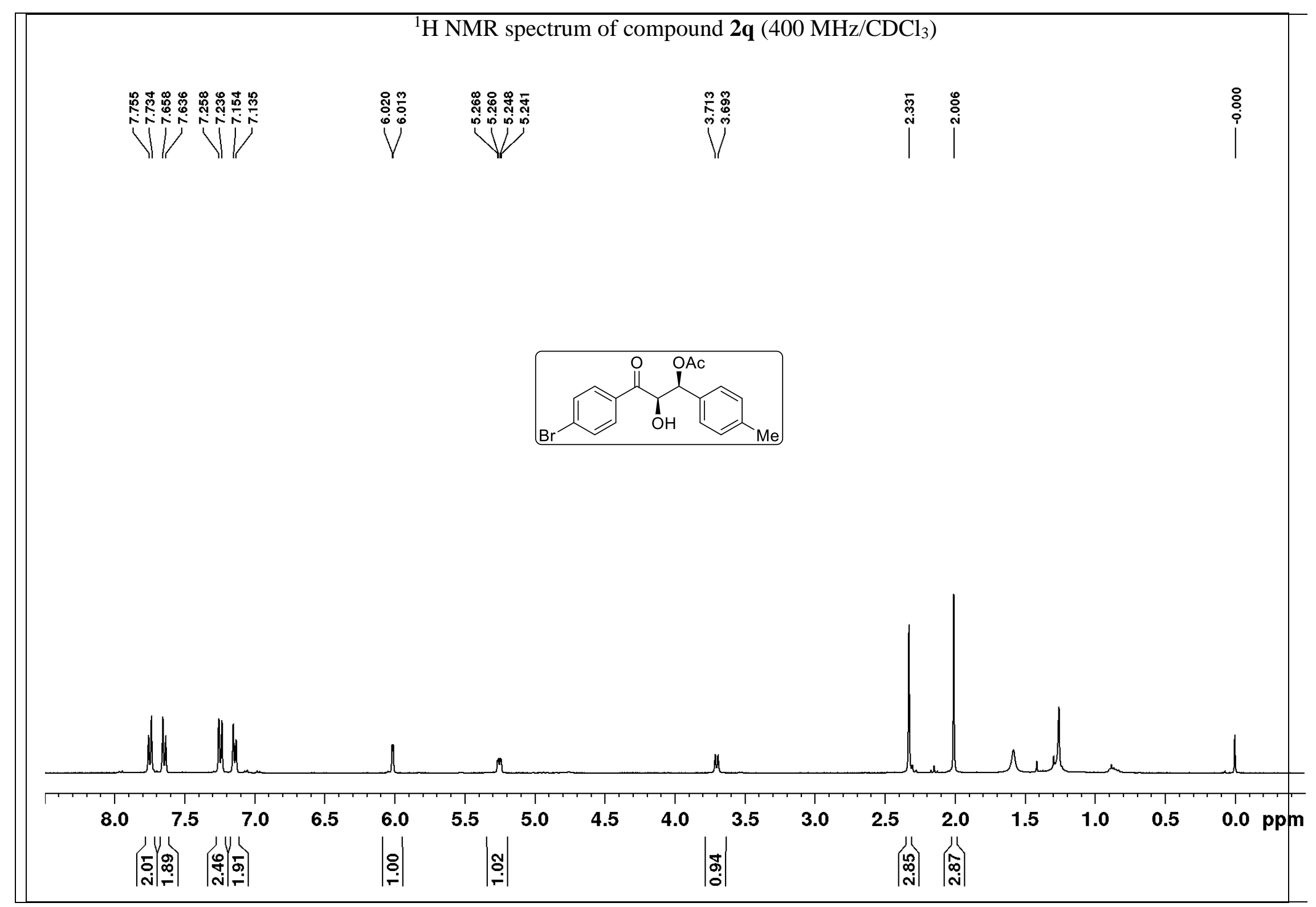




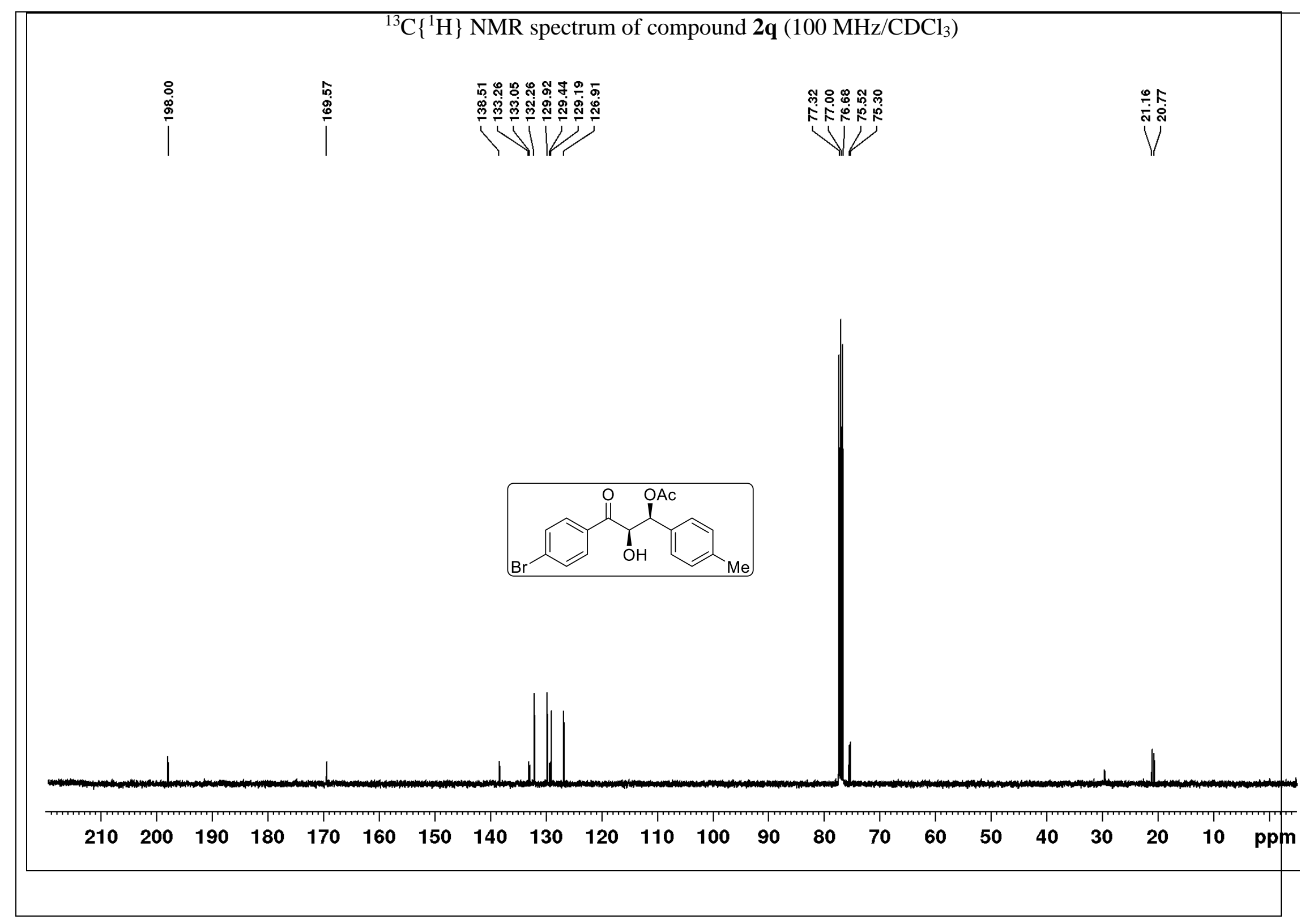




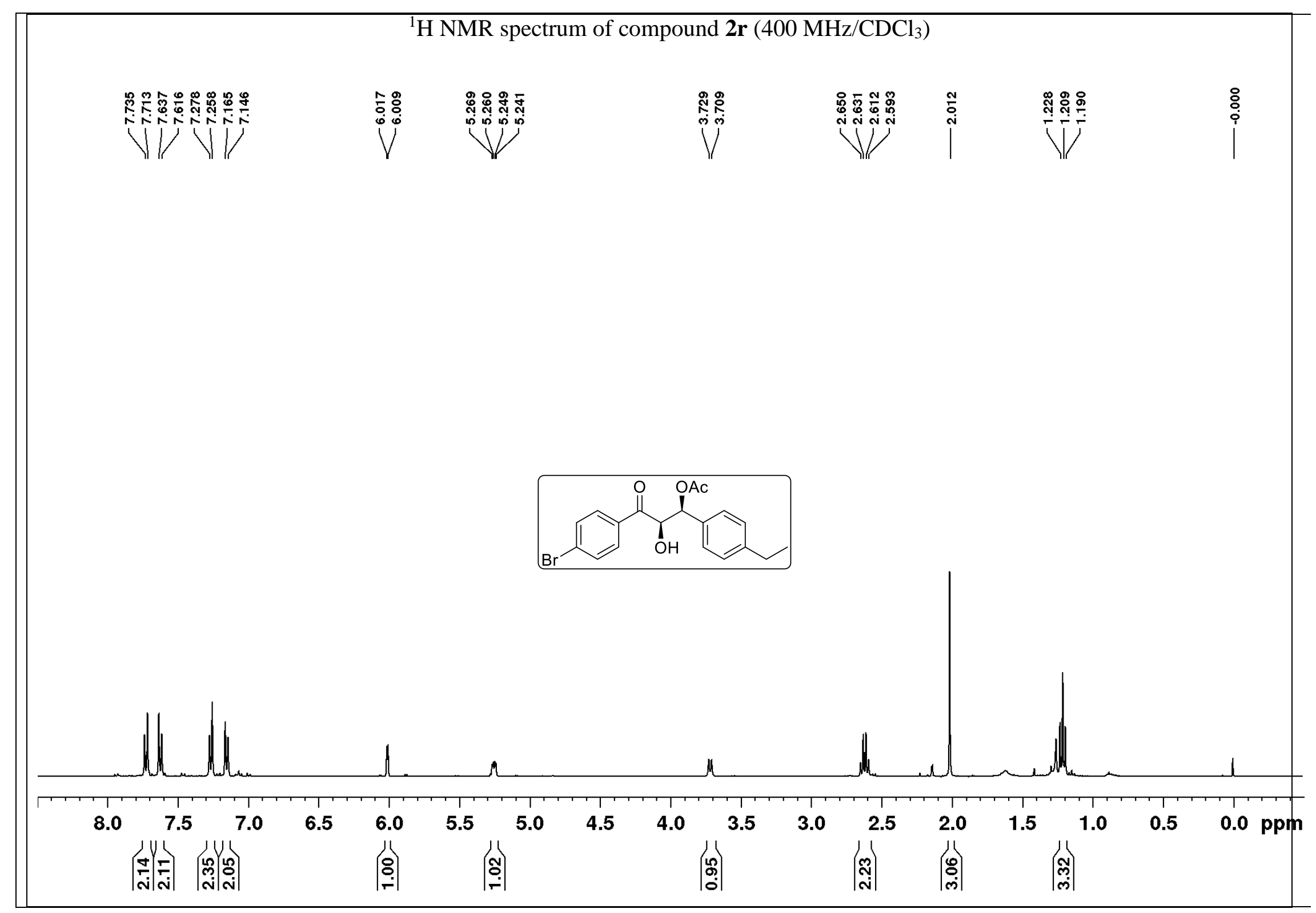




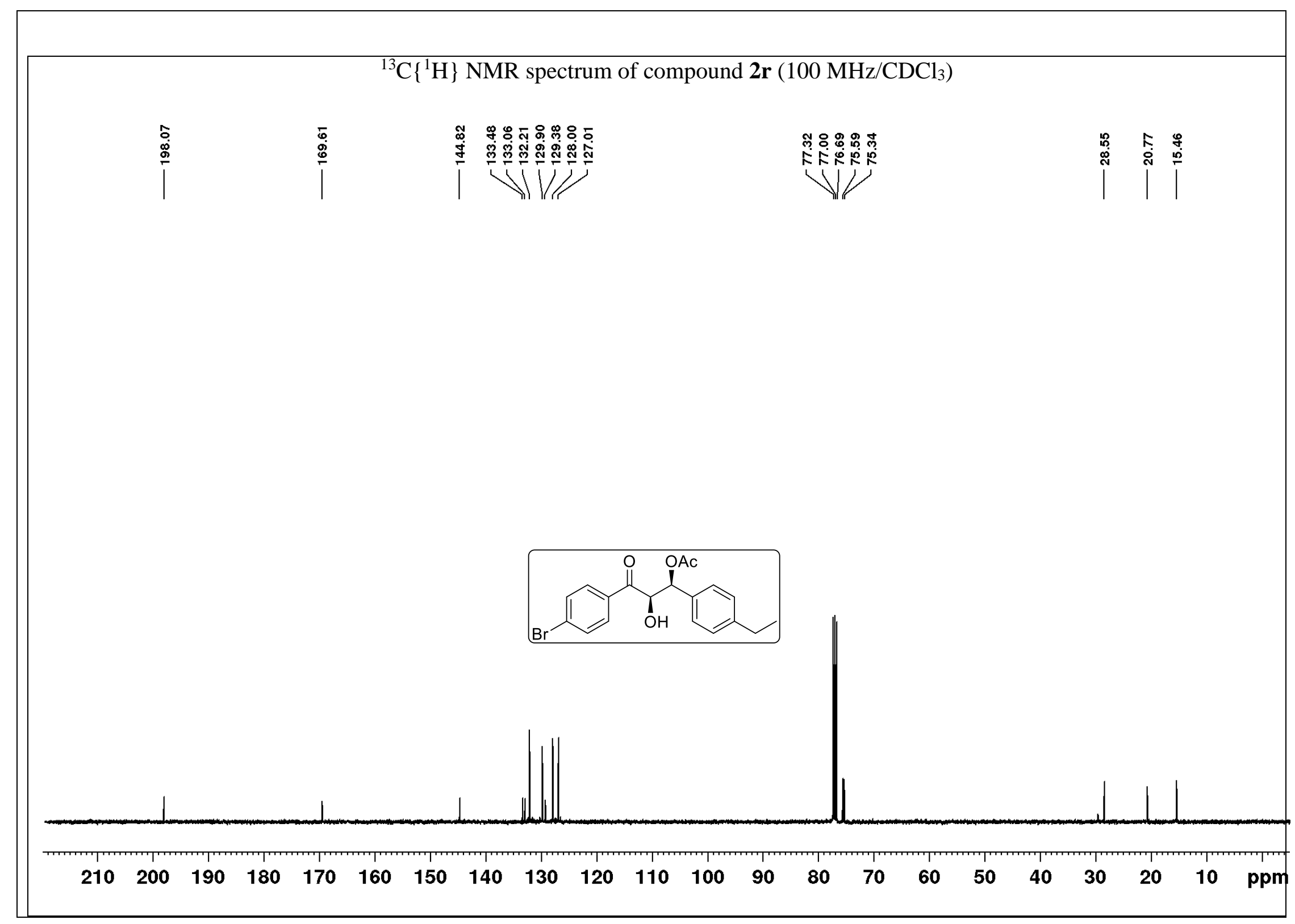




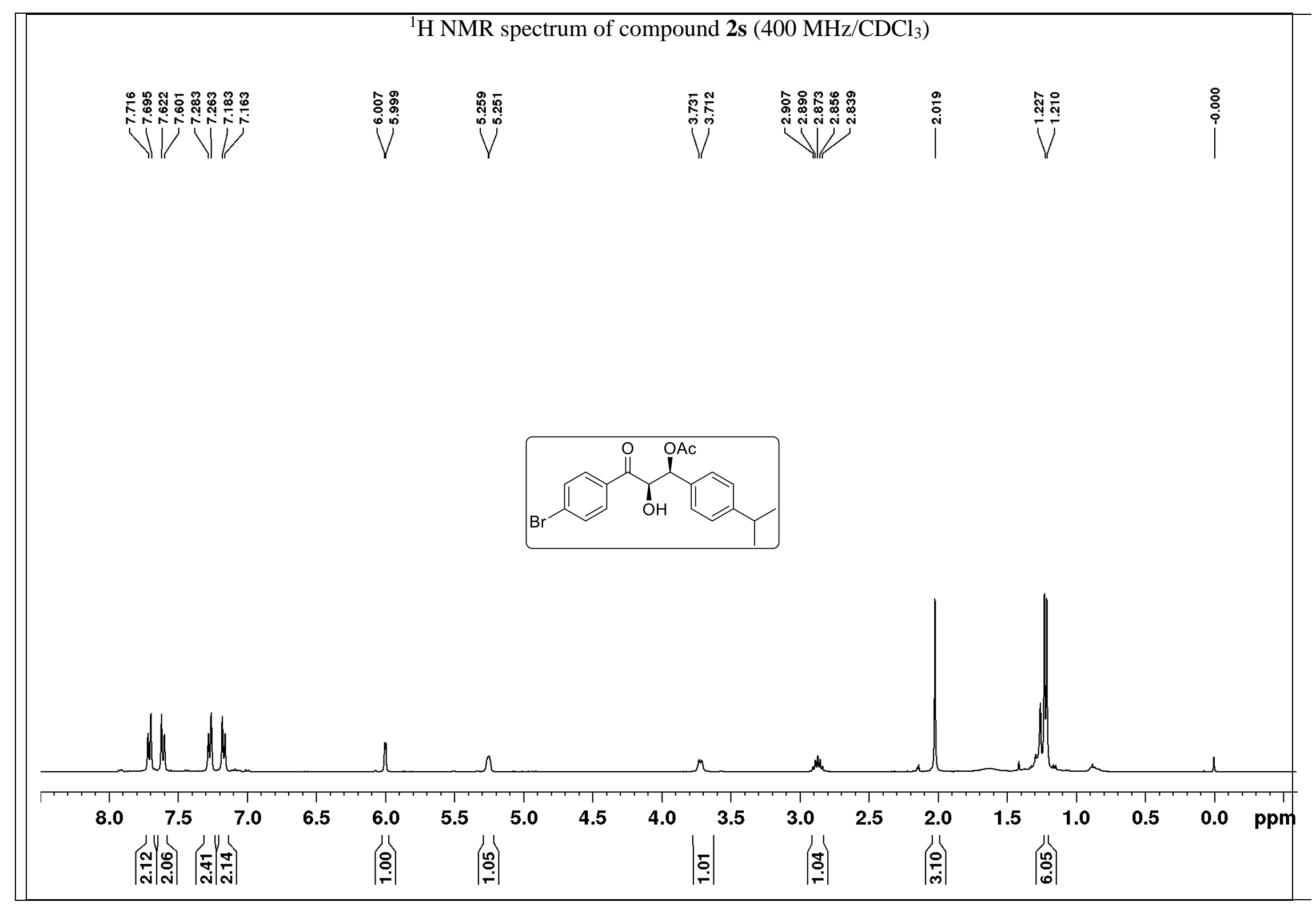




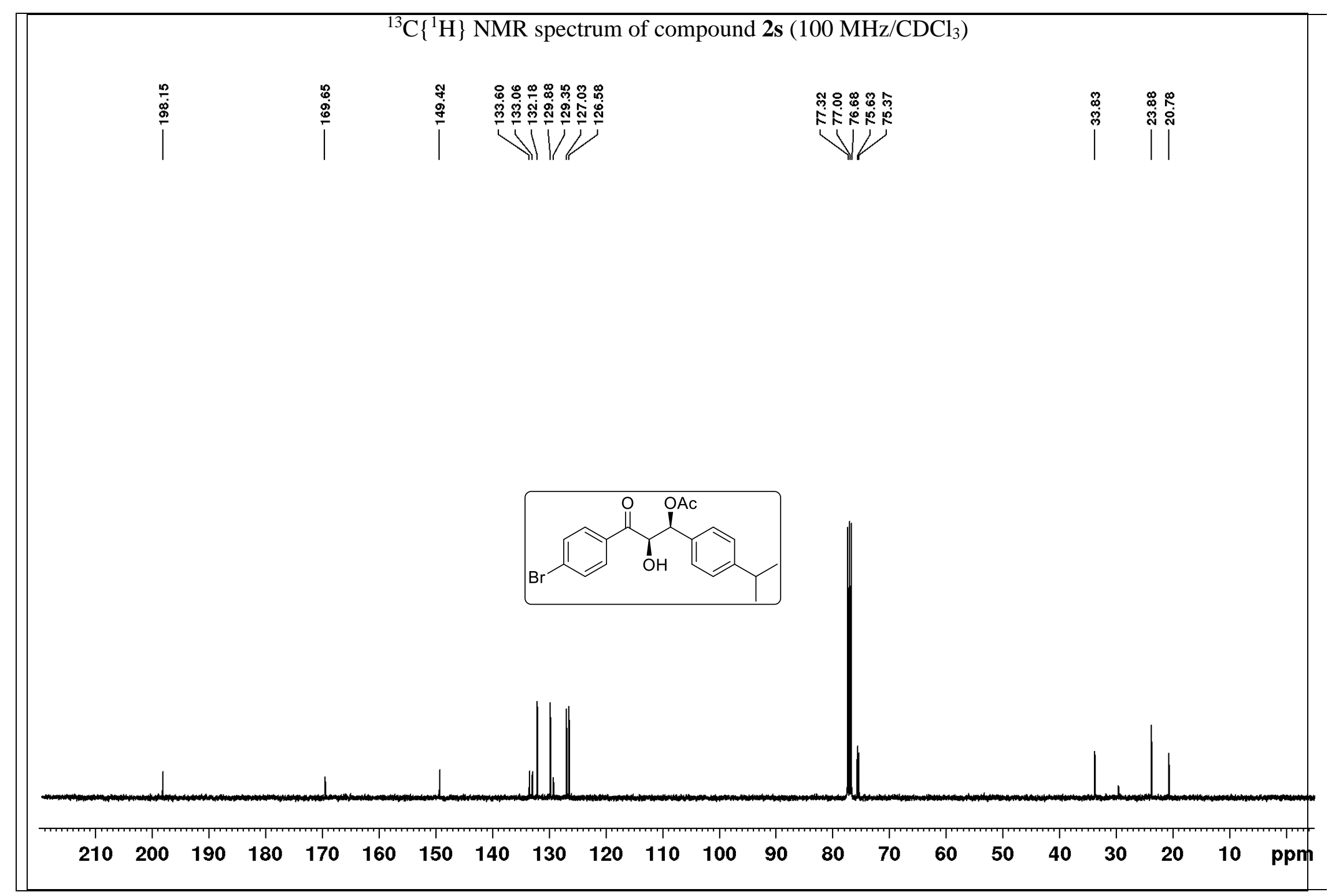




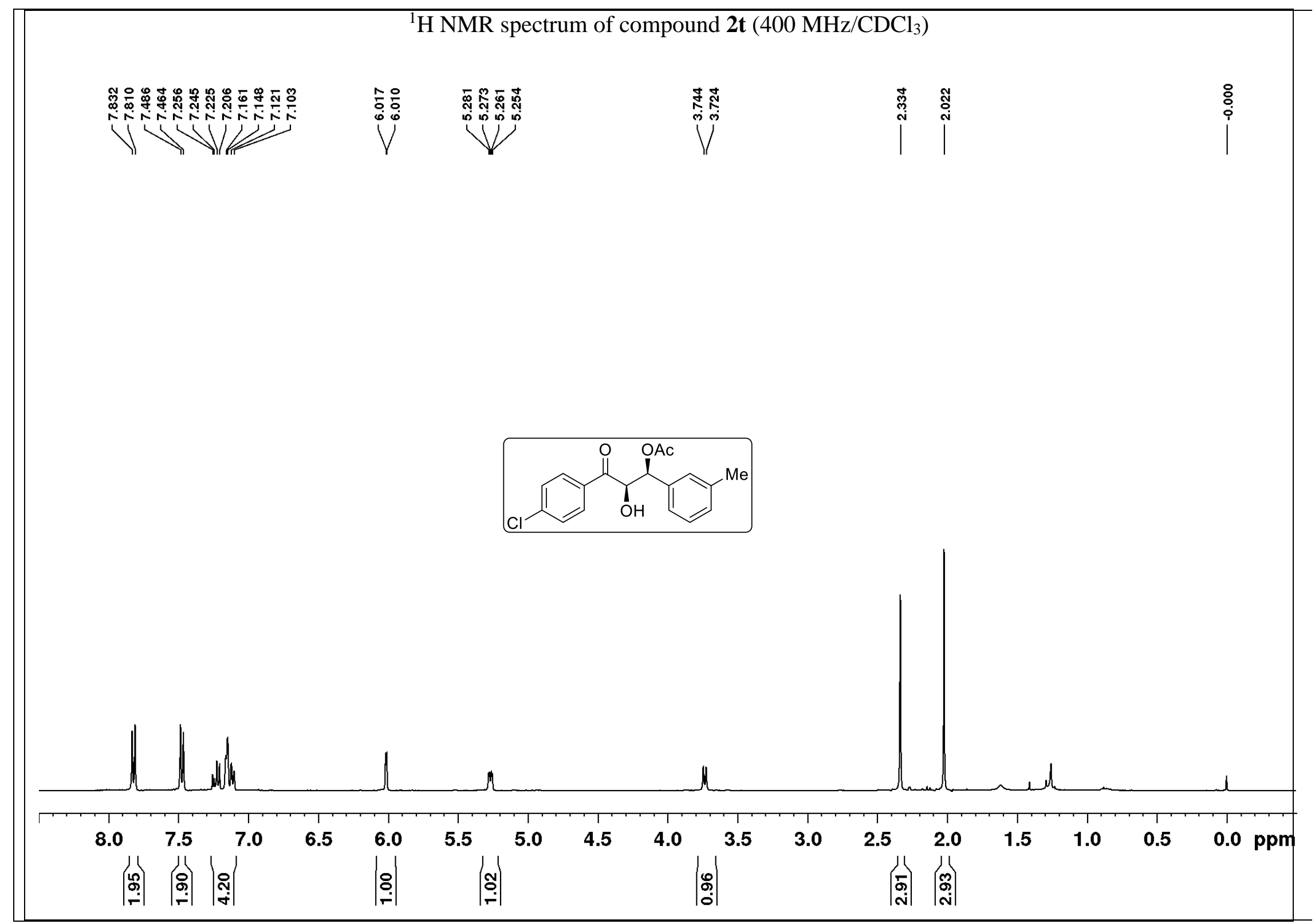

S51 


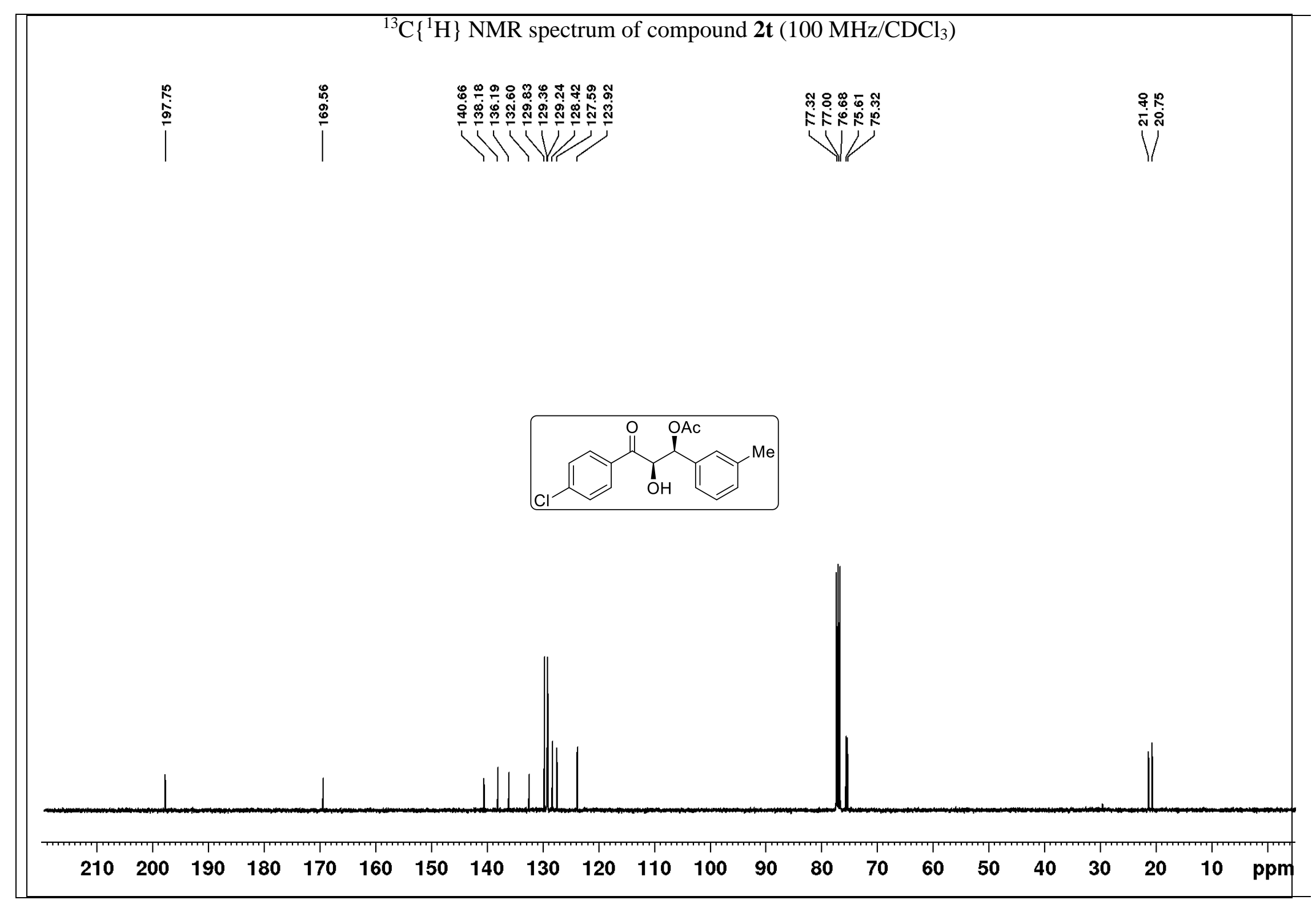




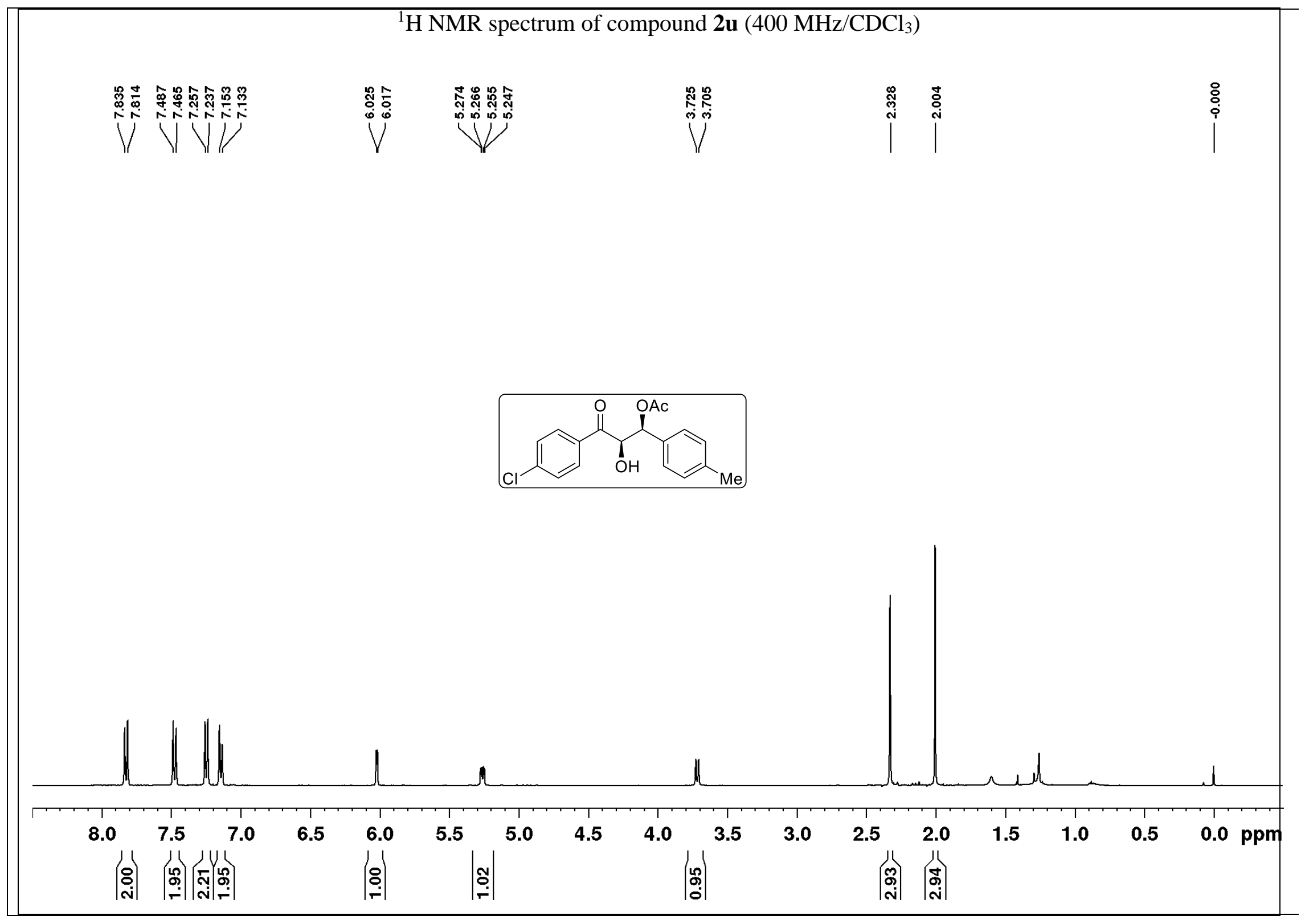

S53 


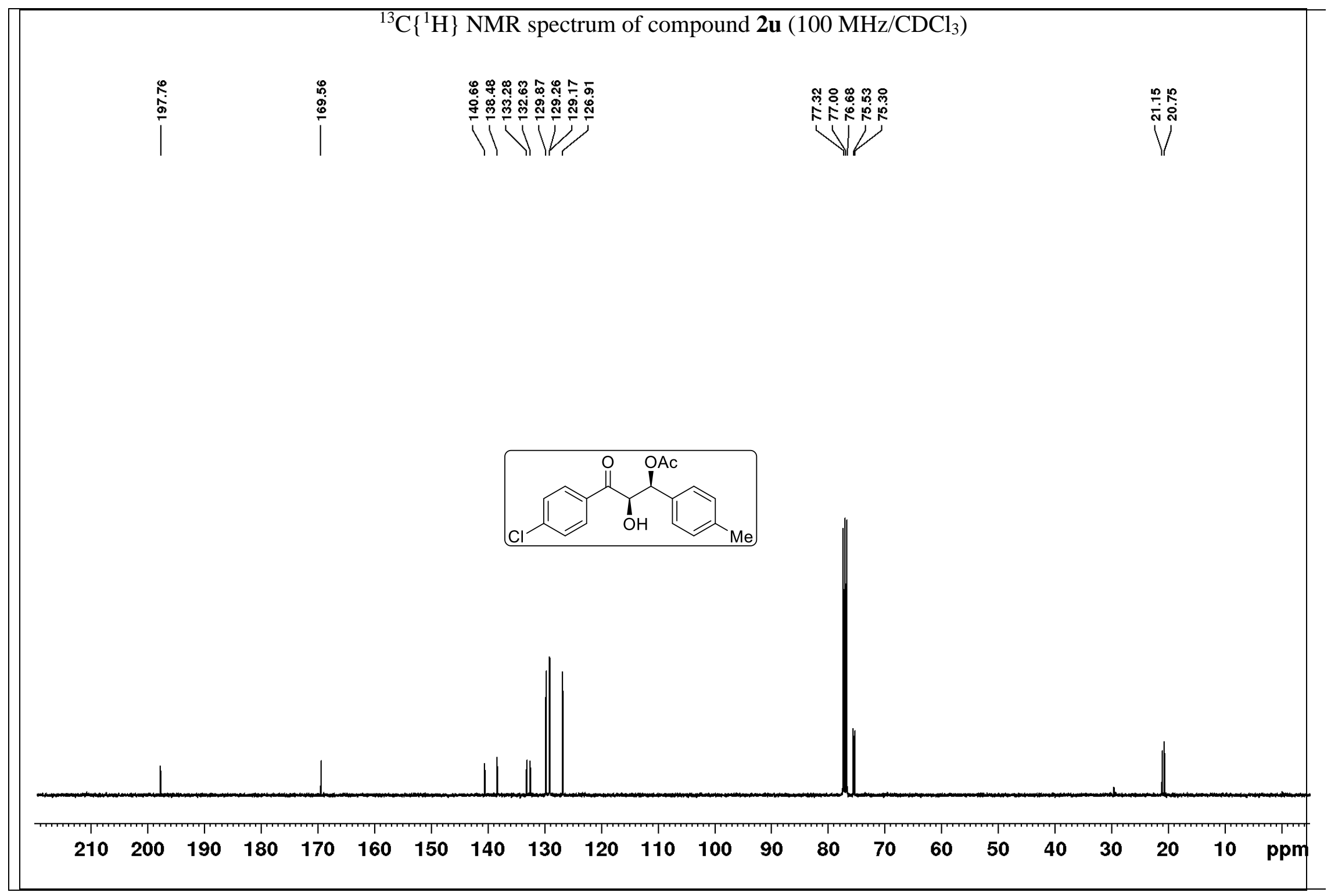

S54 


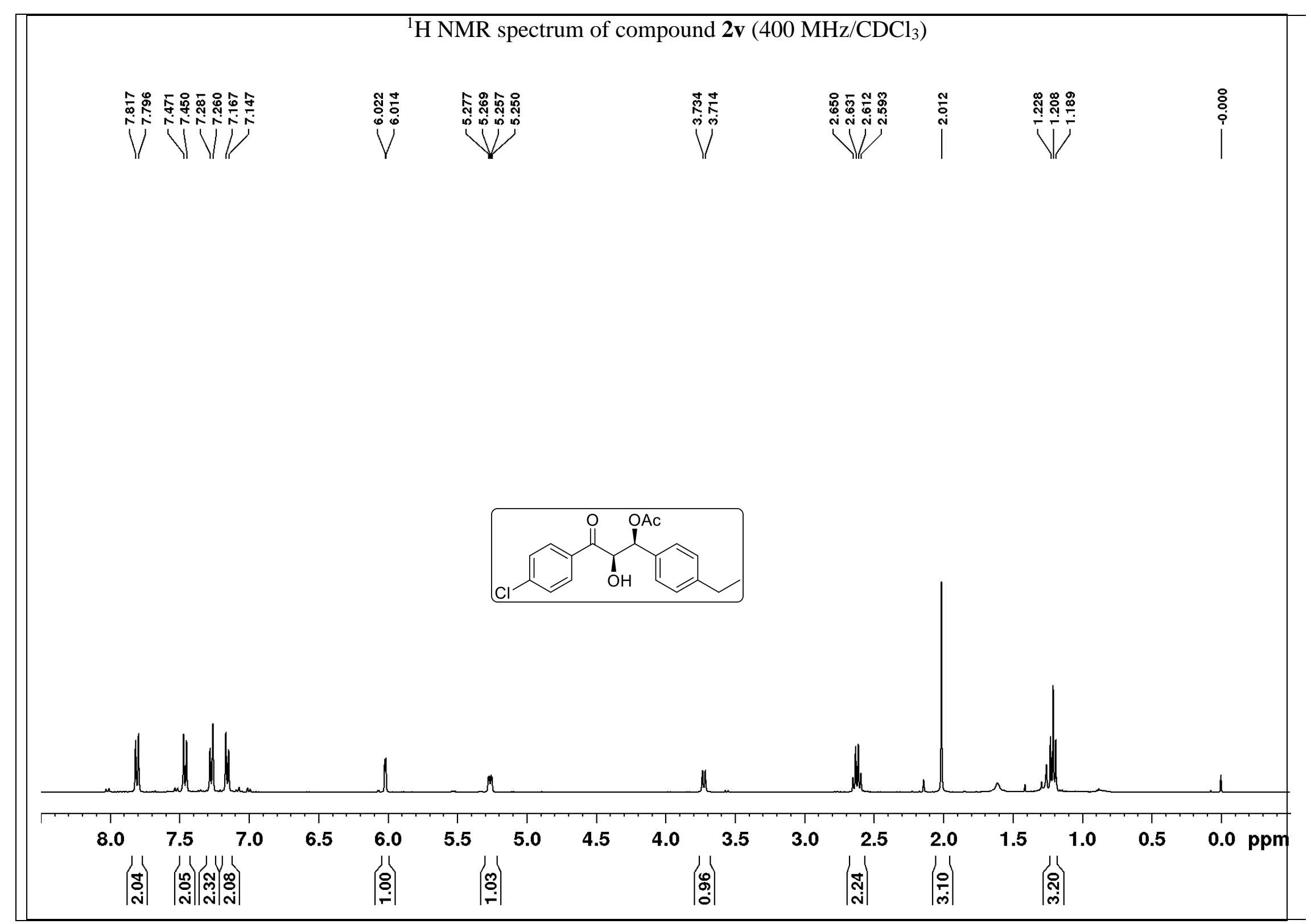




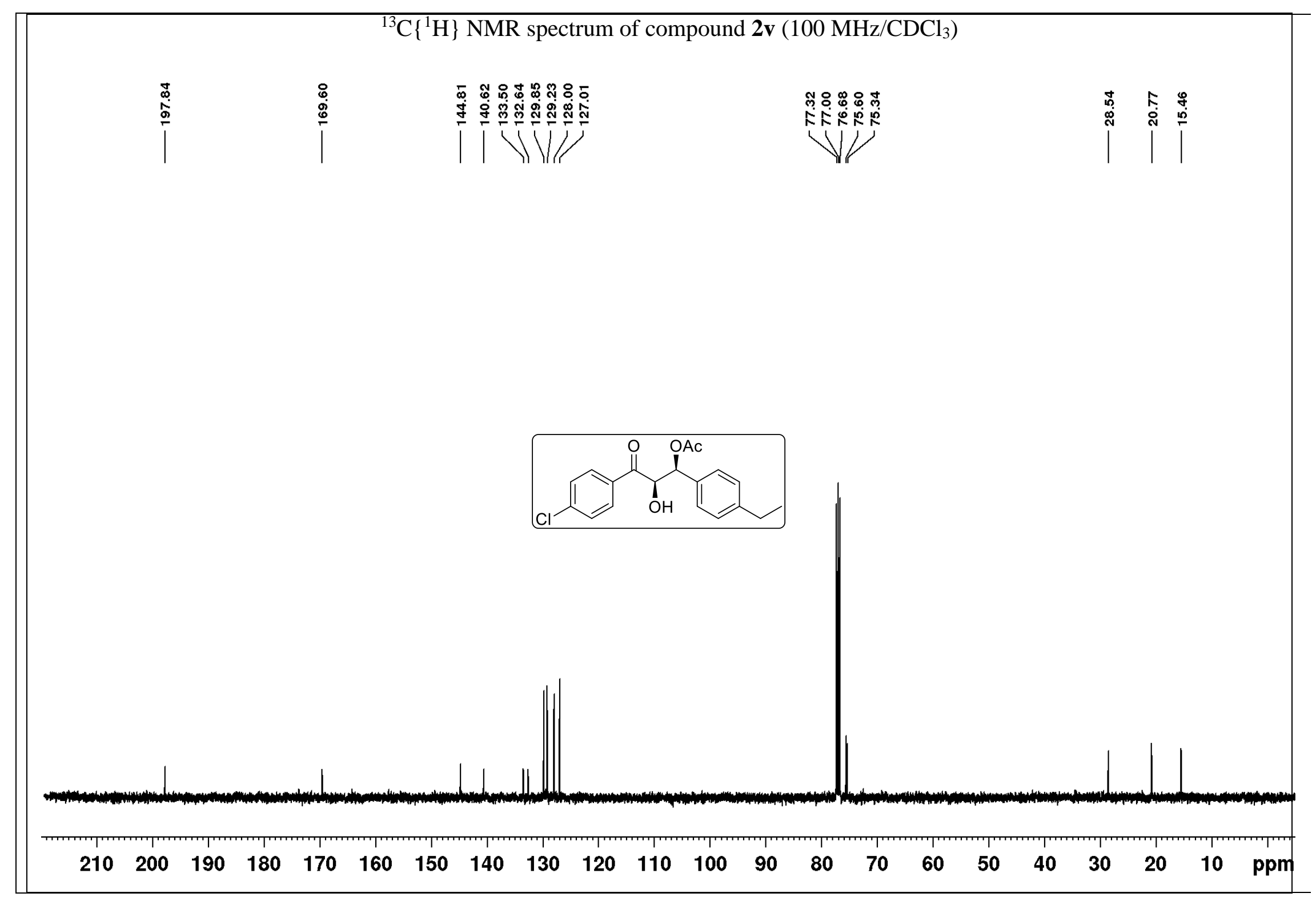




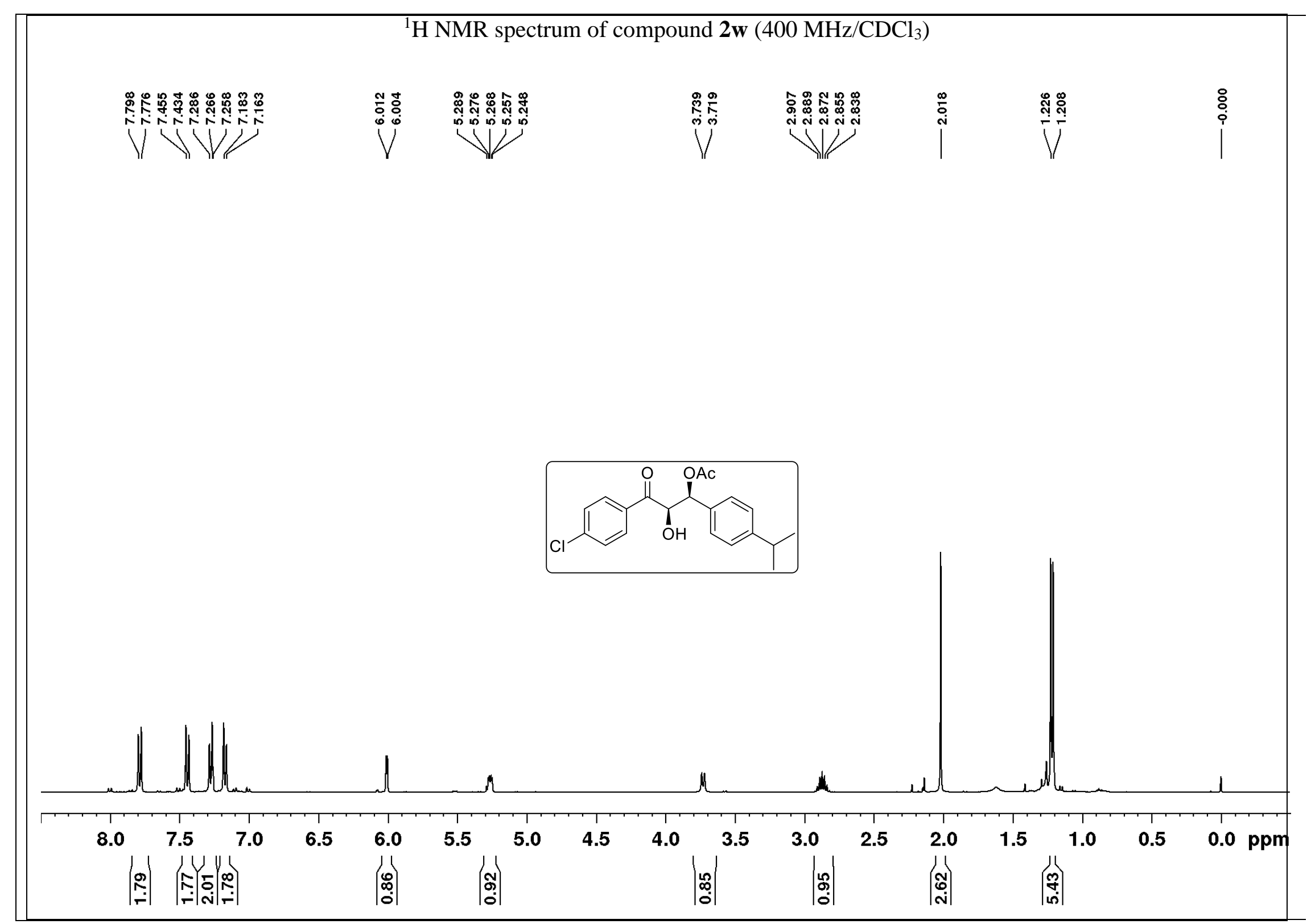




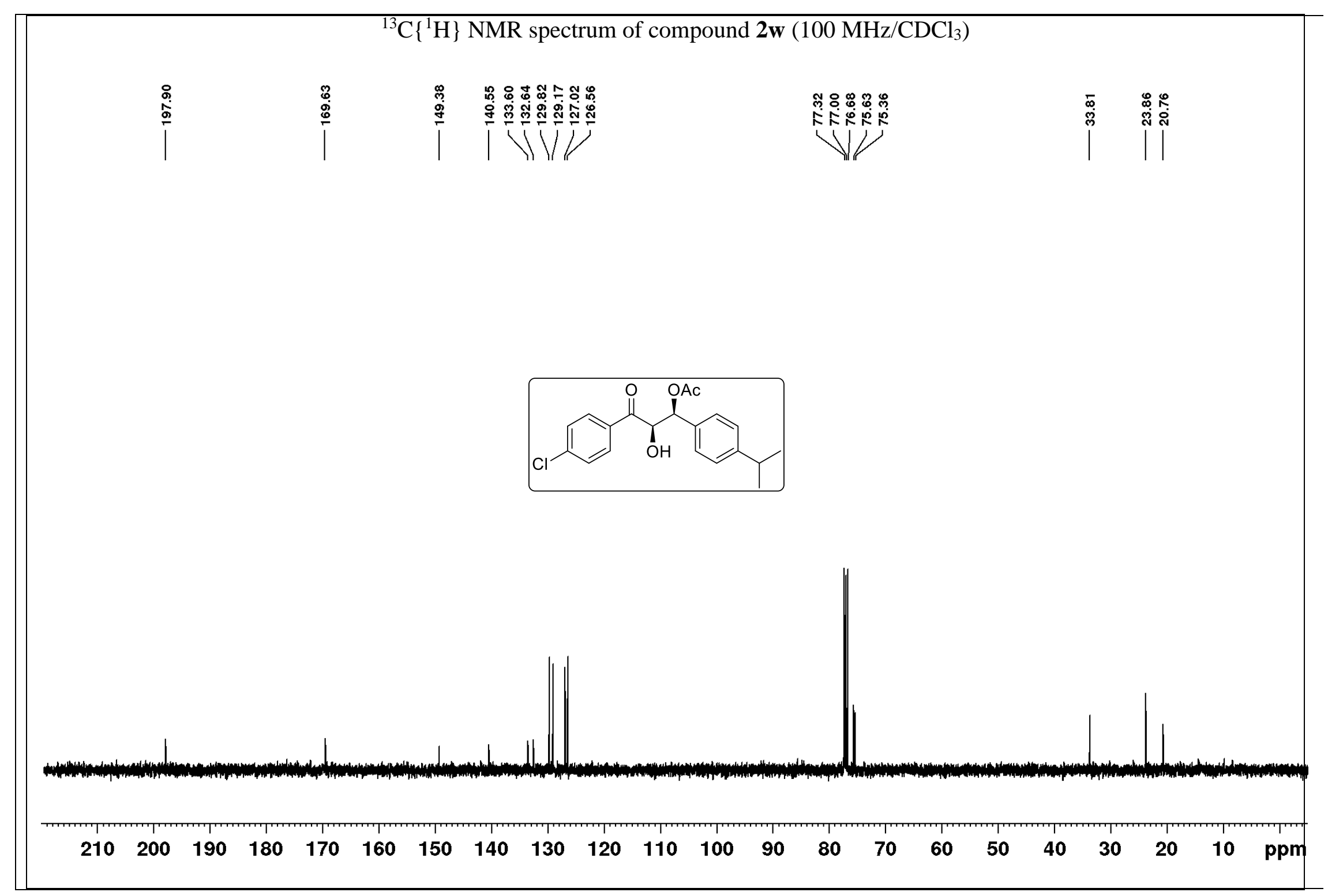




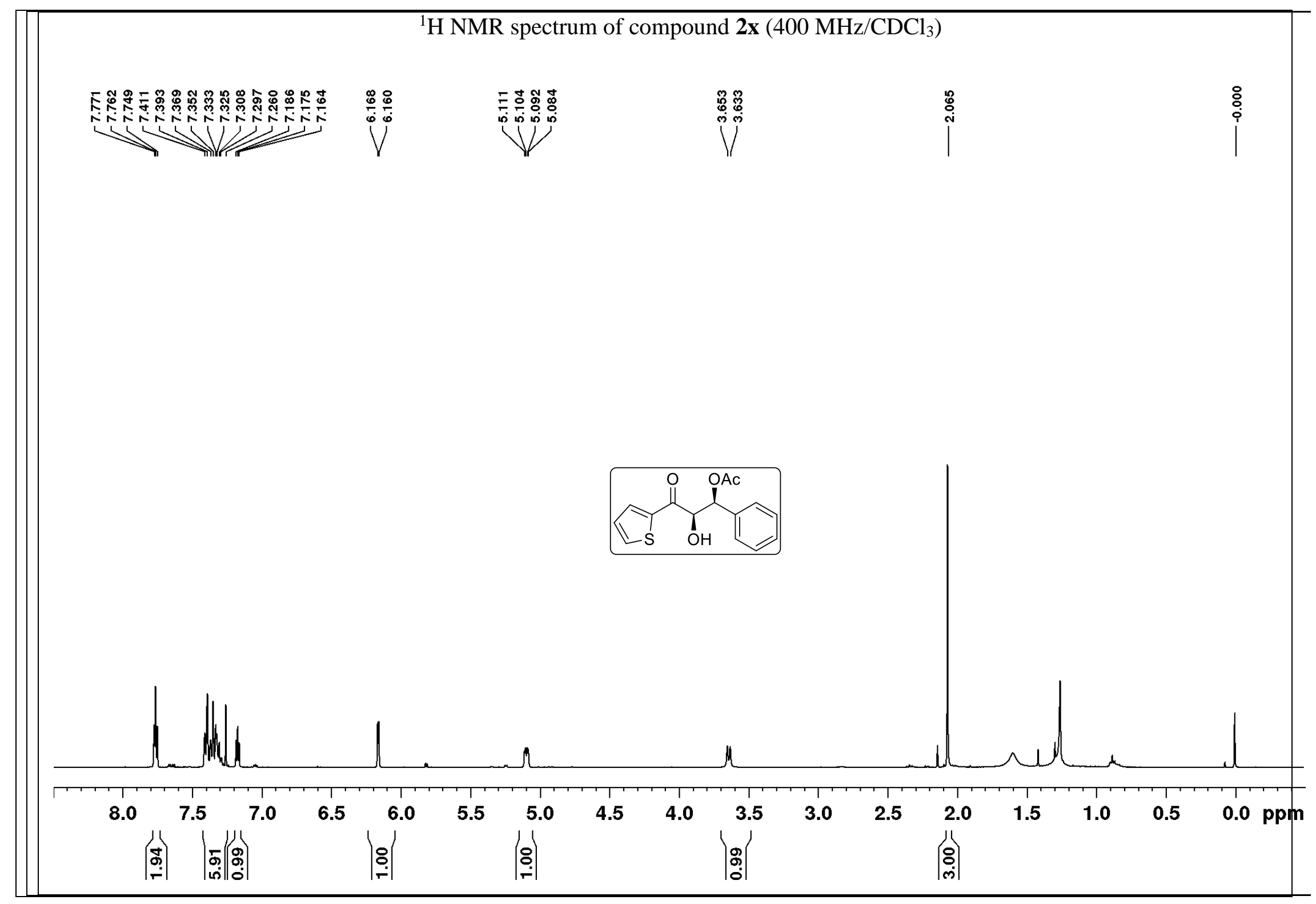




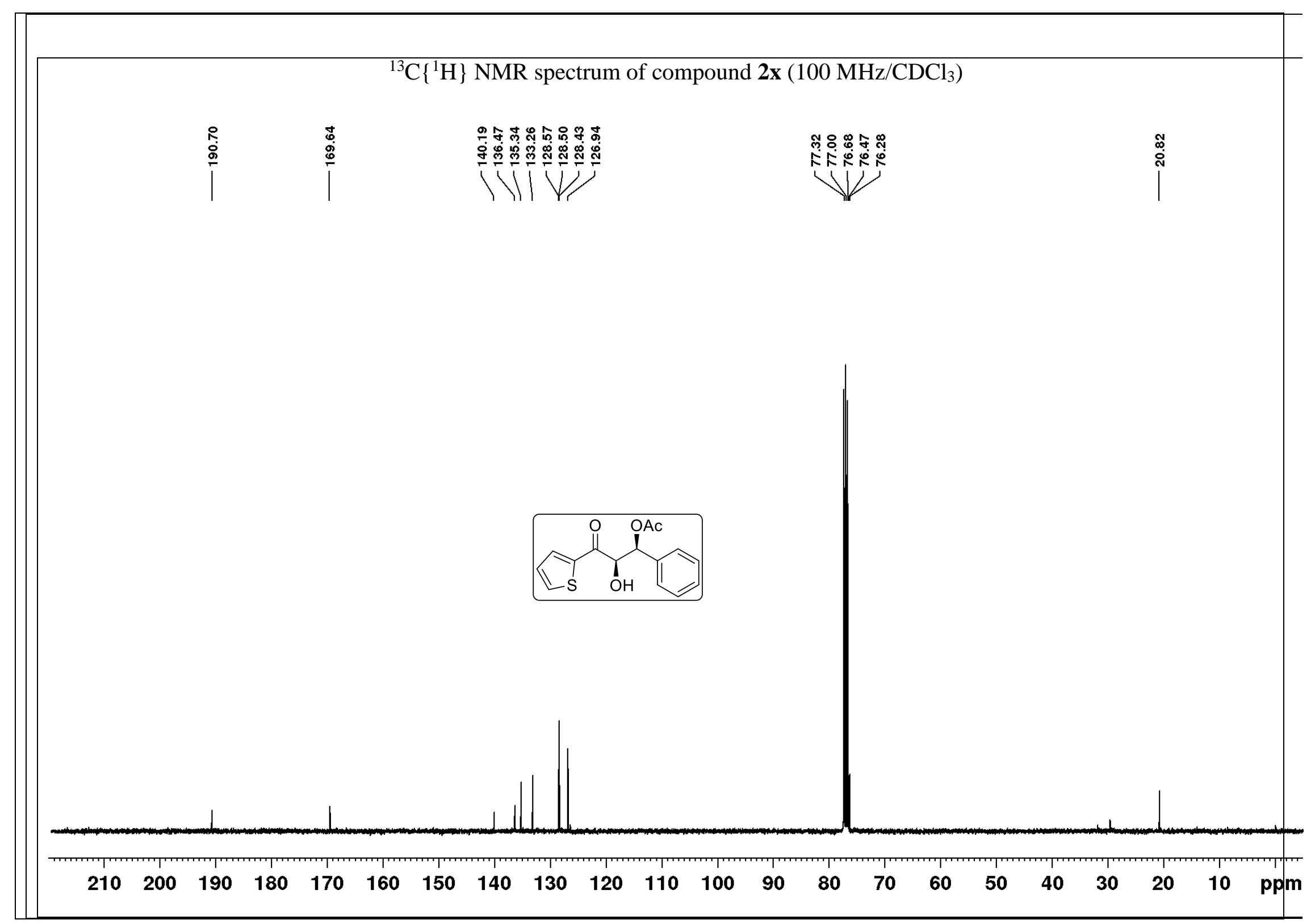




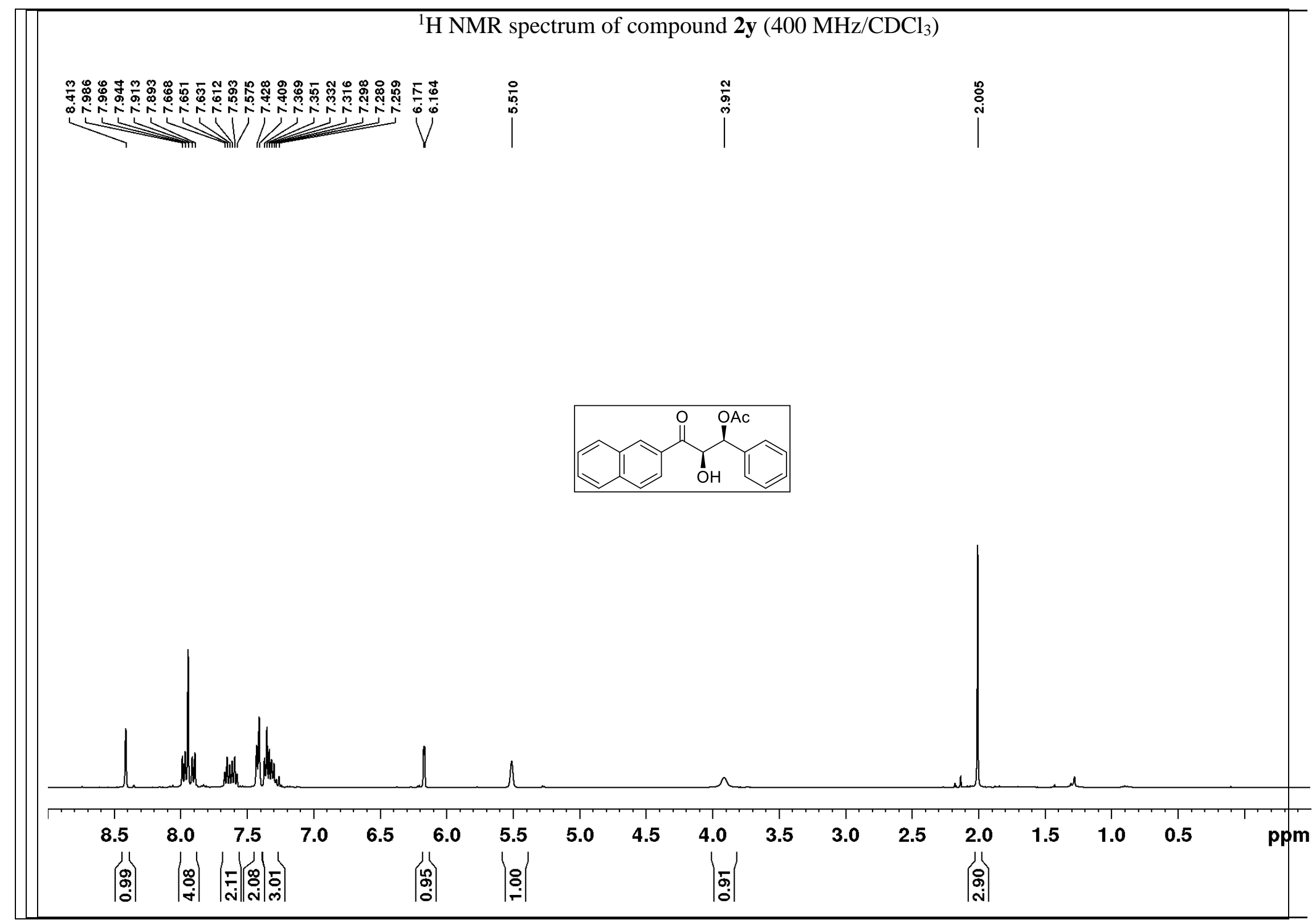




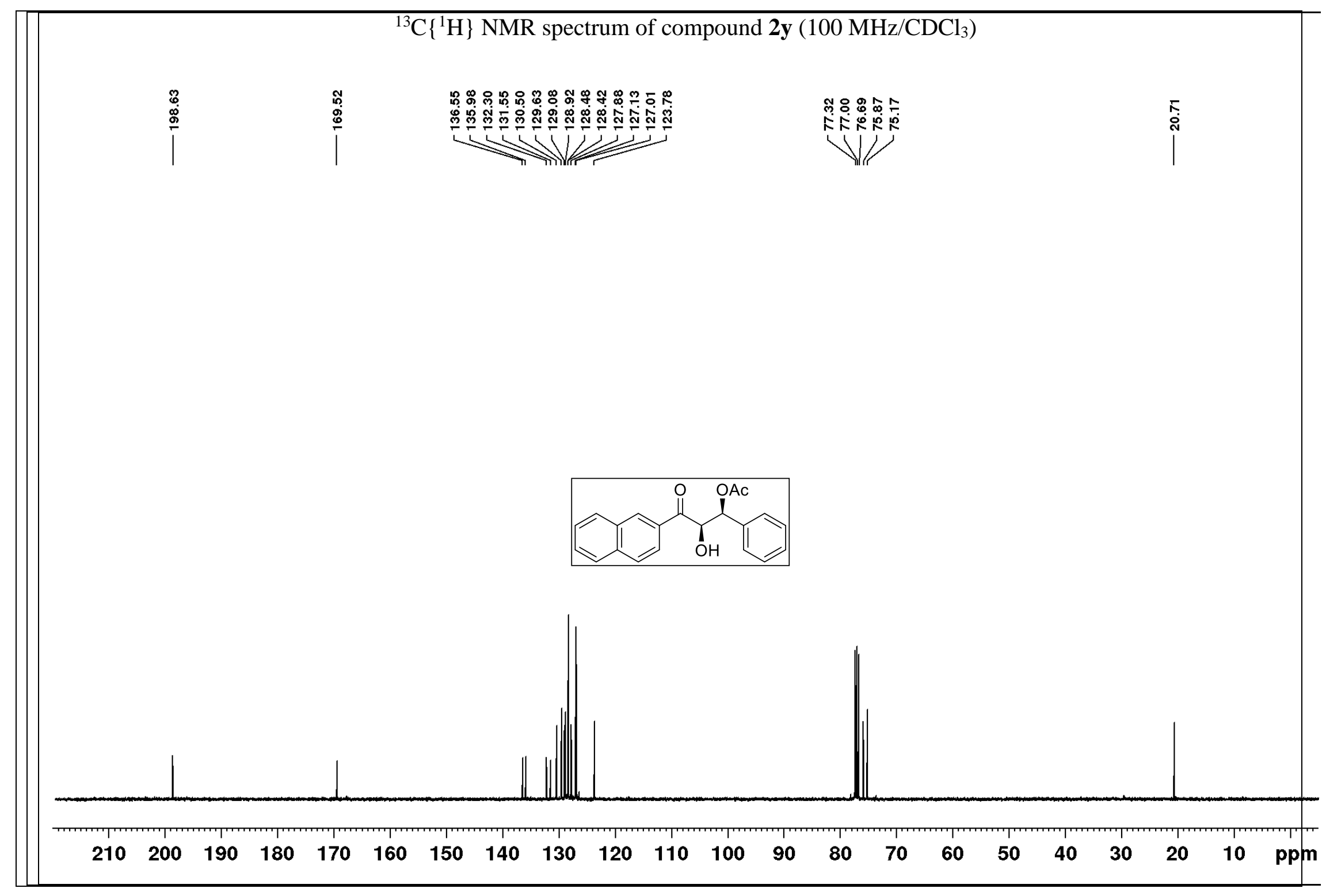




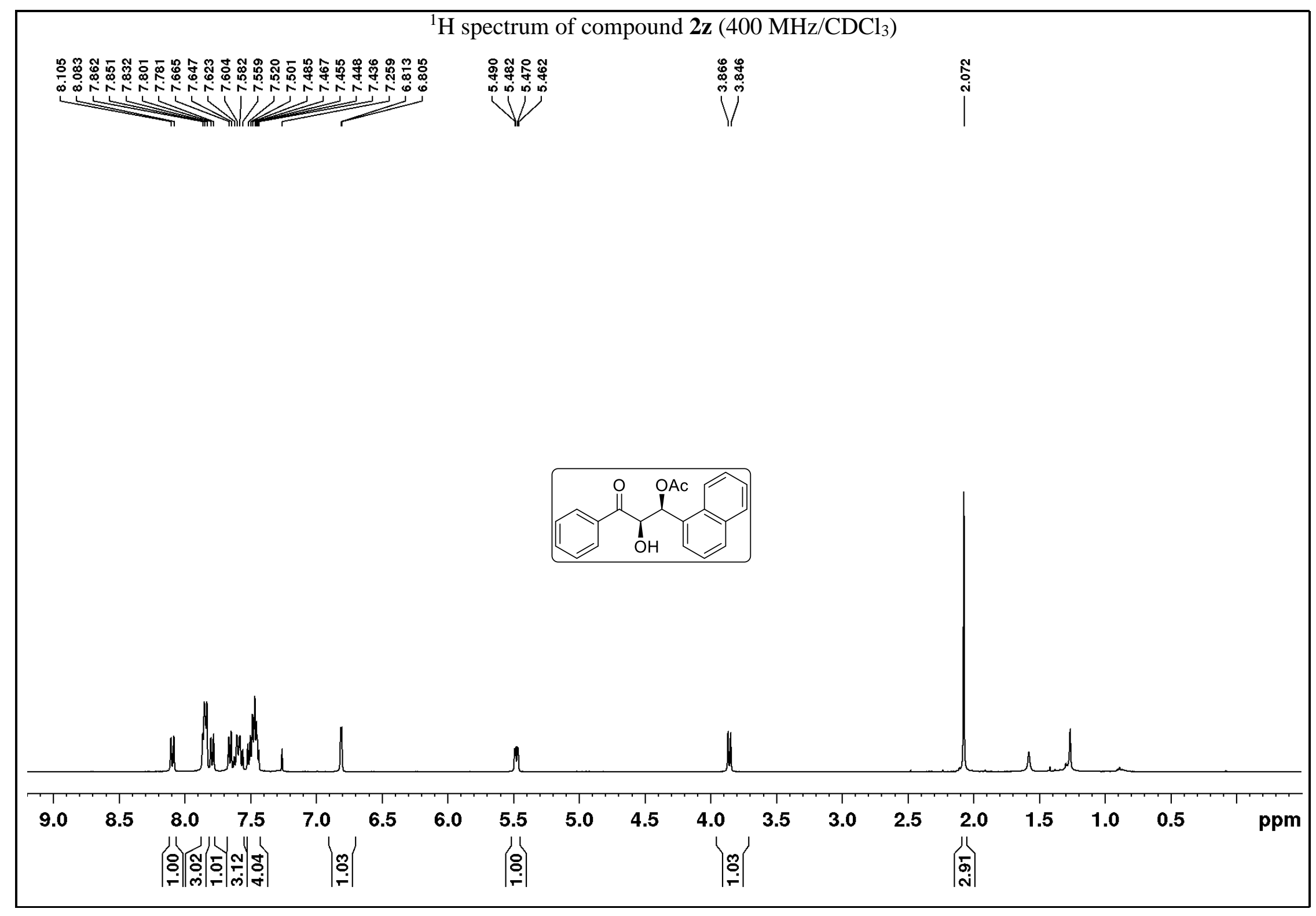




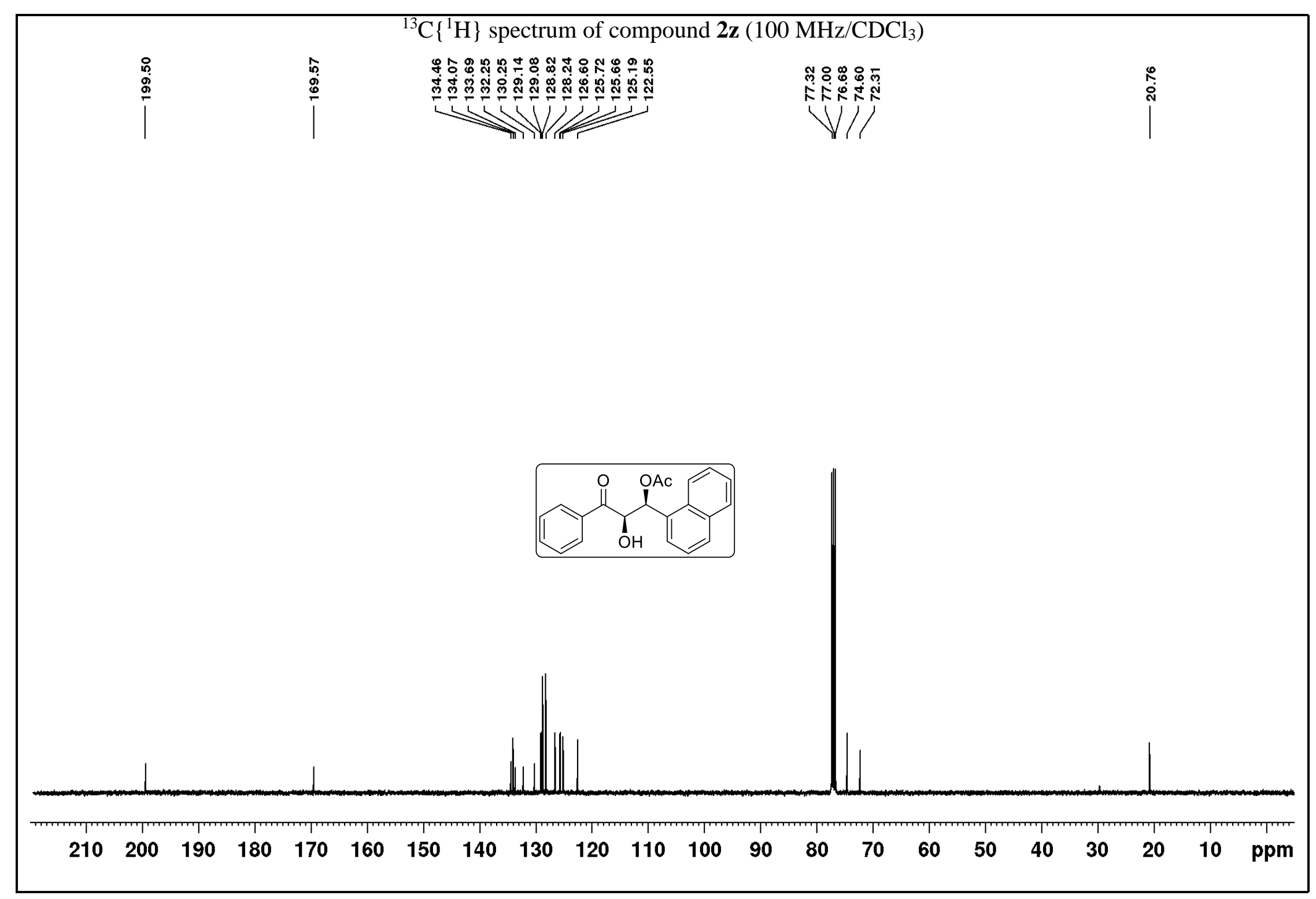




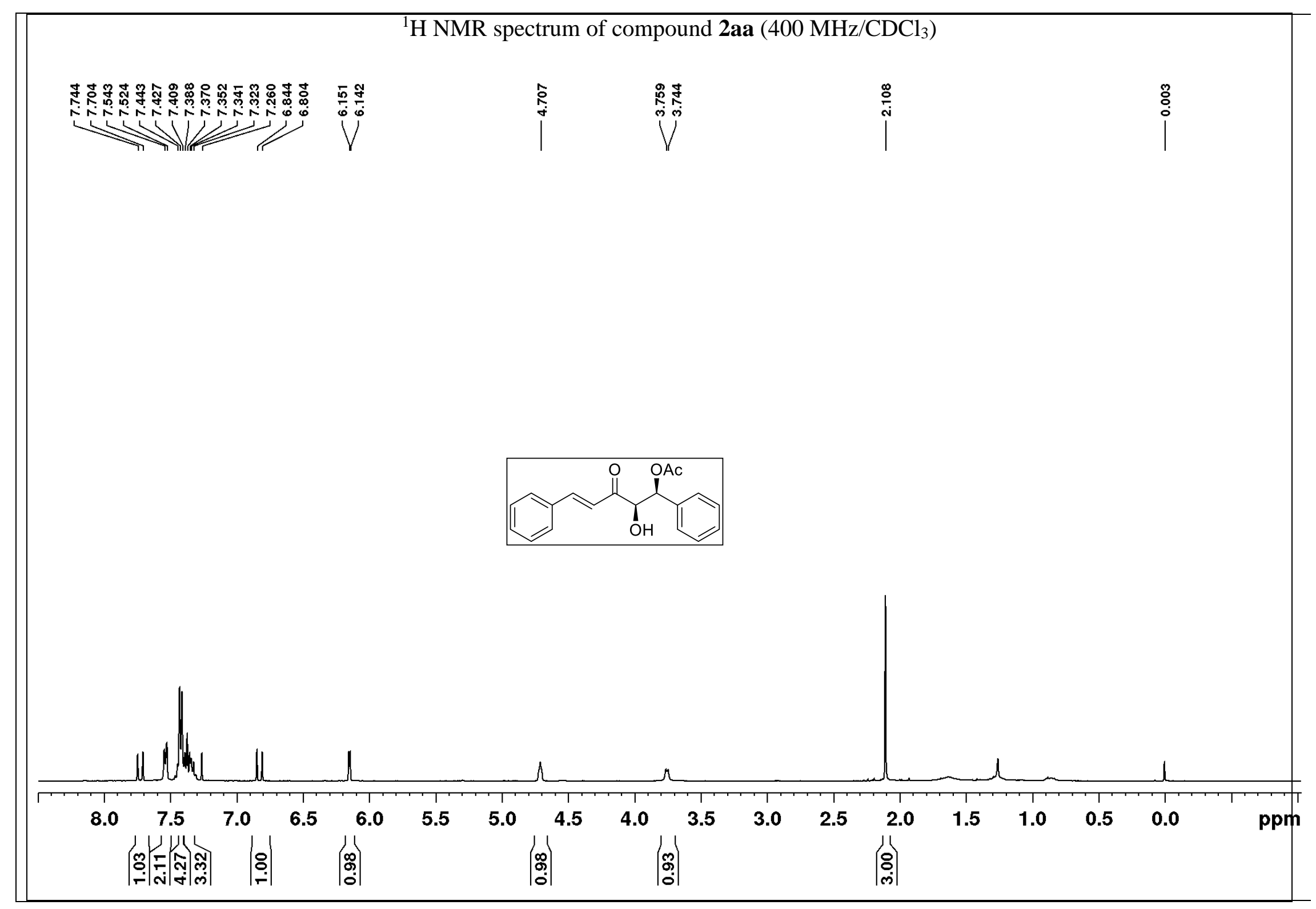




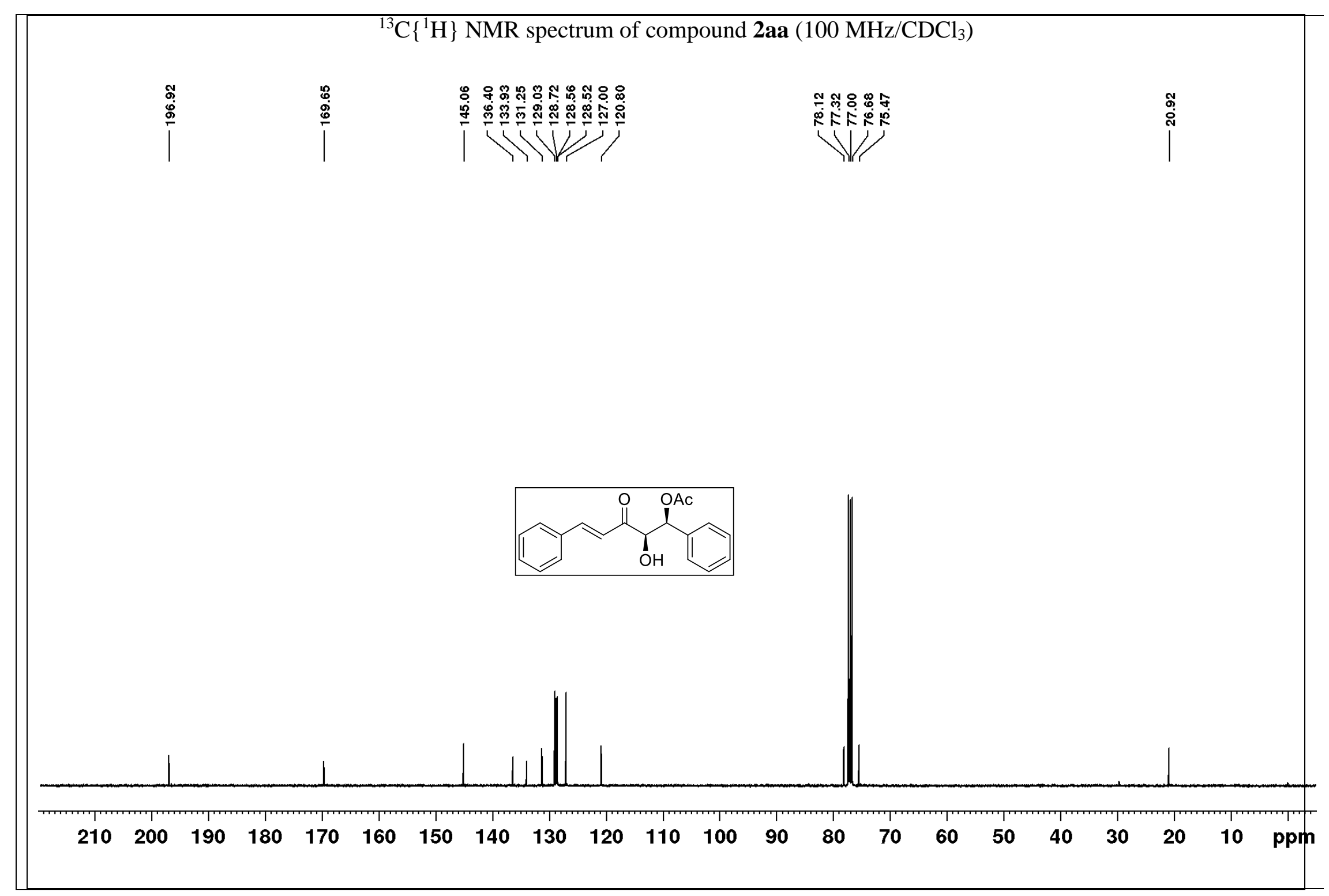




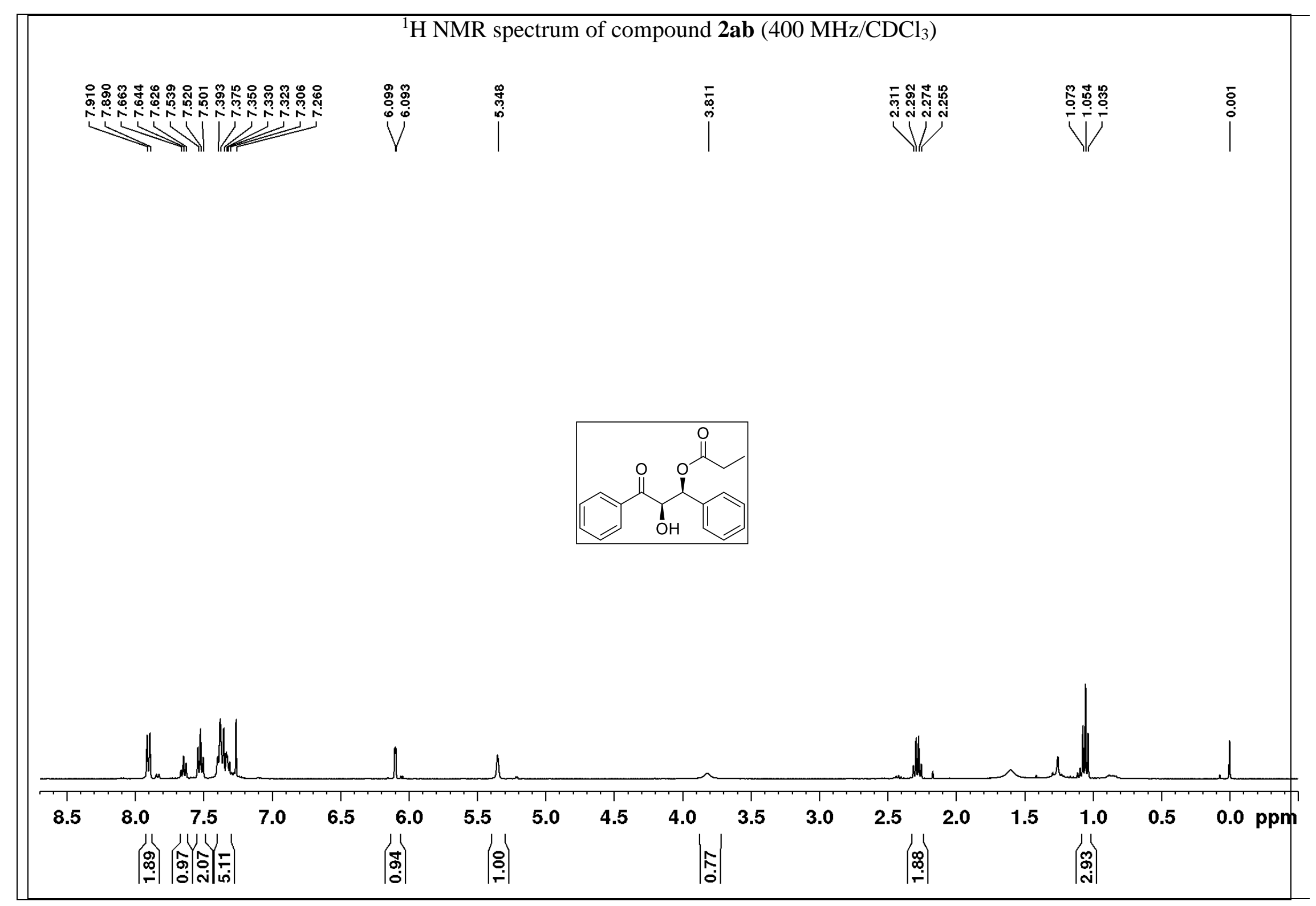




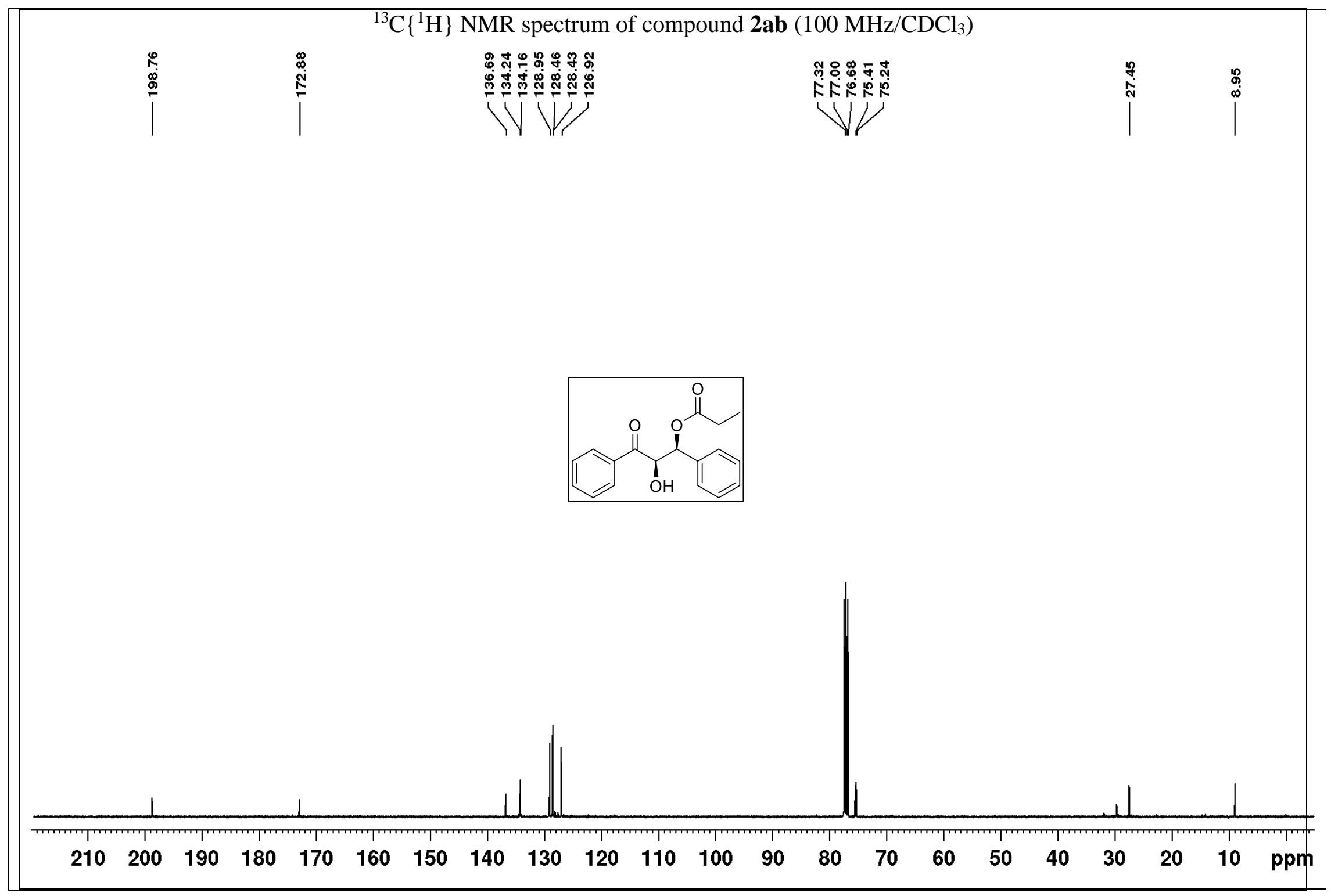

S68 


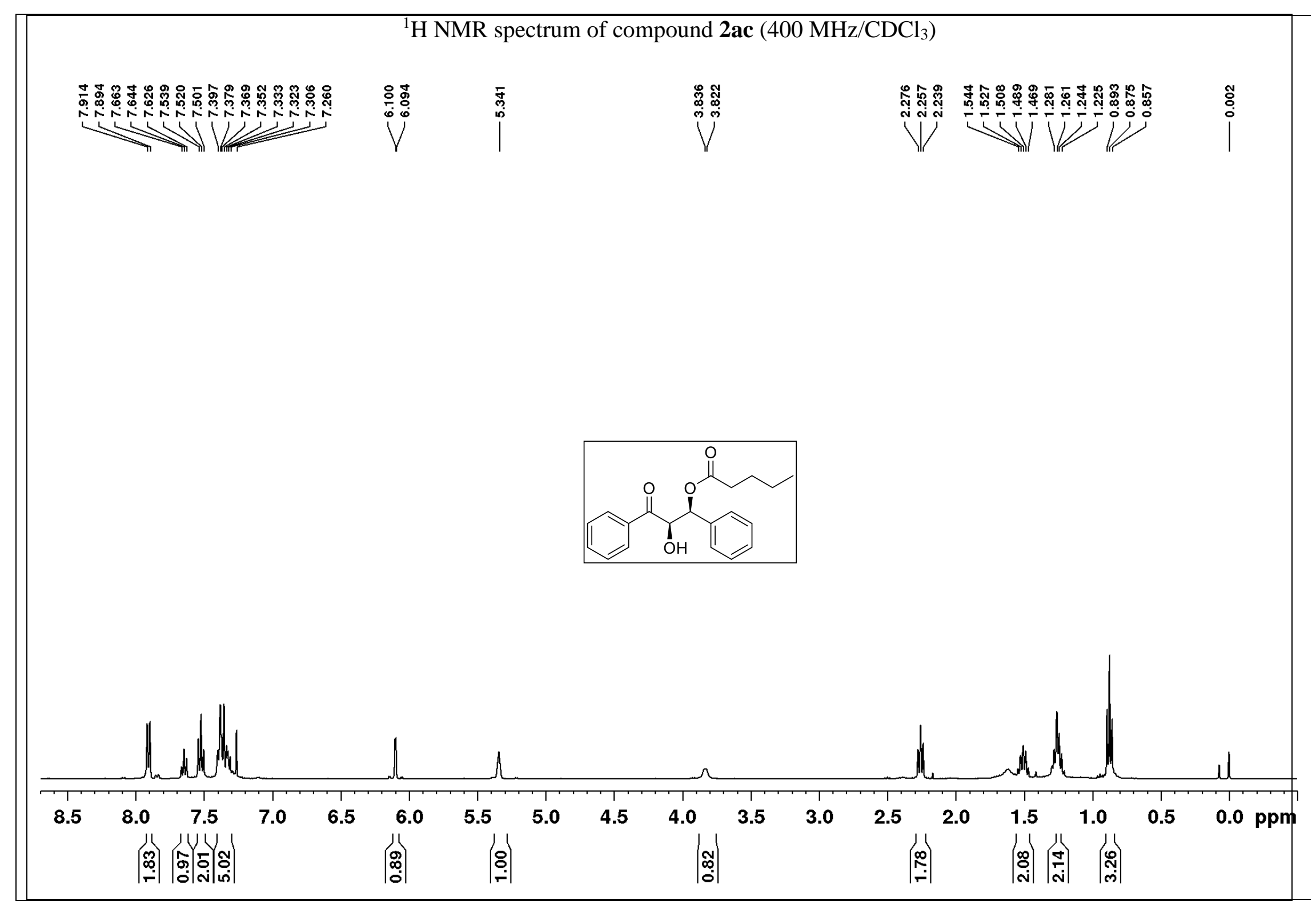




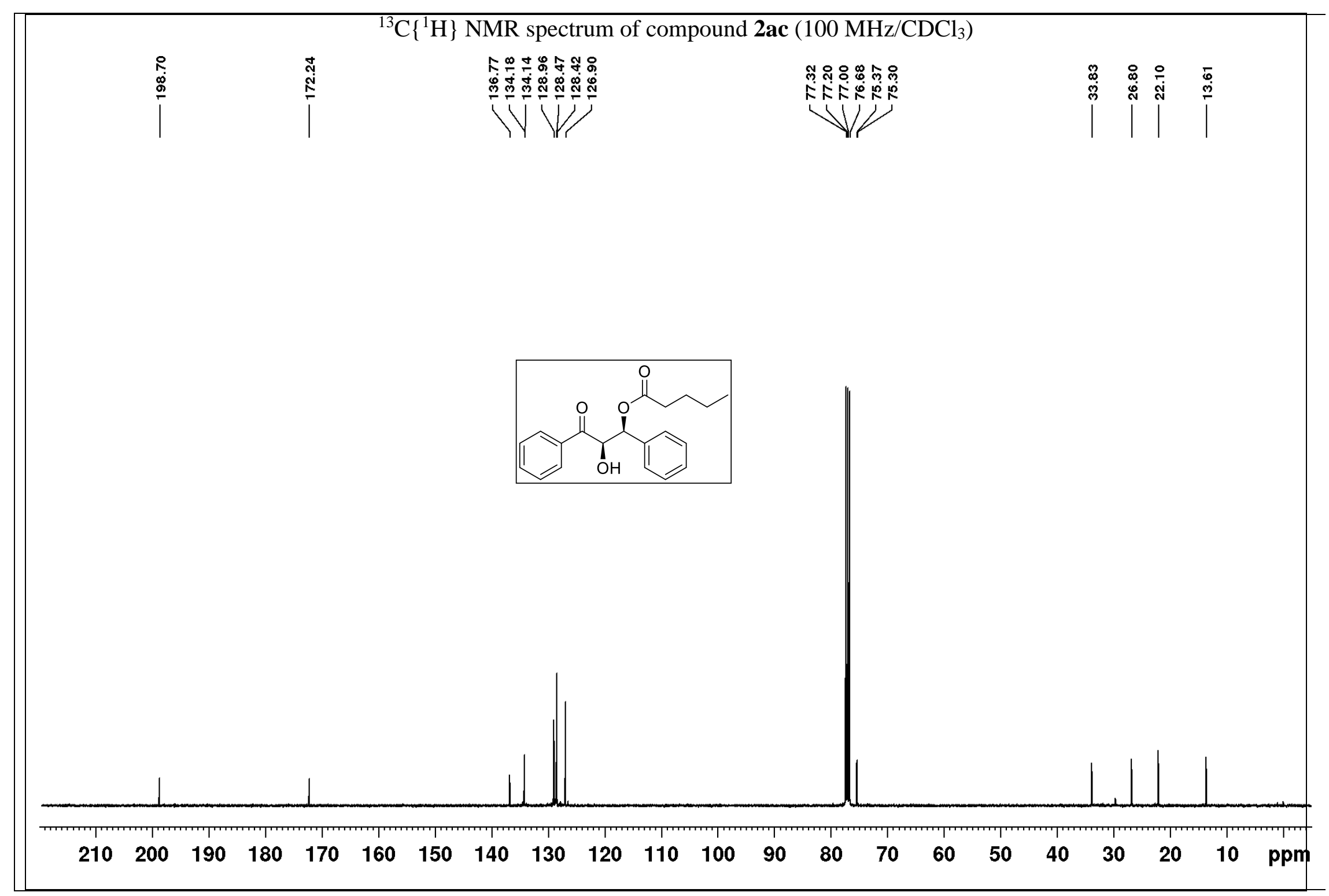


VIII. HPLC spectra of Hydroxy-oxyacylation products, 2:<smiles>O=C(c1ccccc1)c1ccccc1C(O)C(O)C(=O)O</smiles>

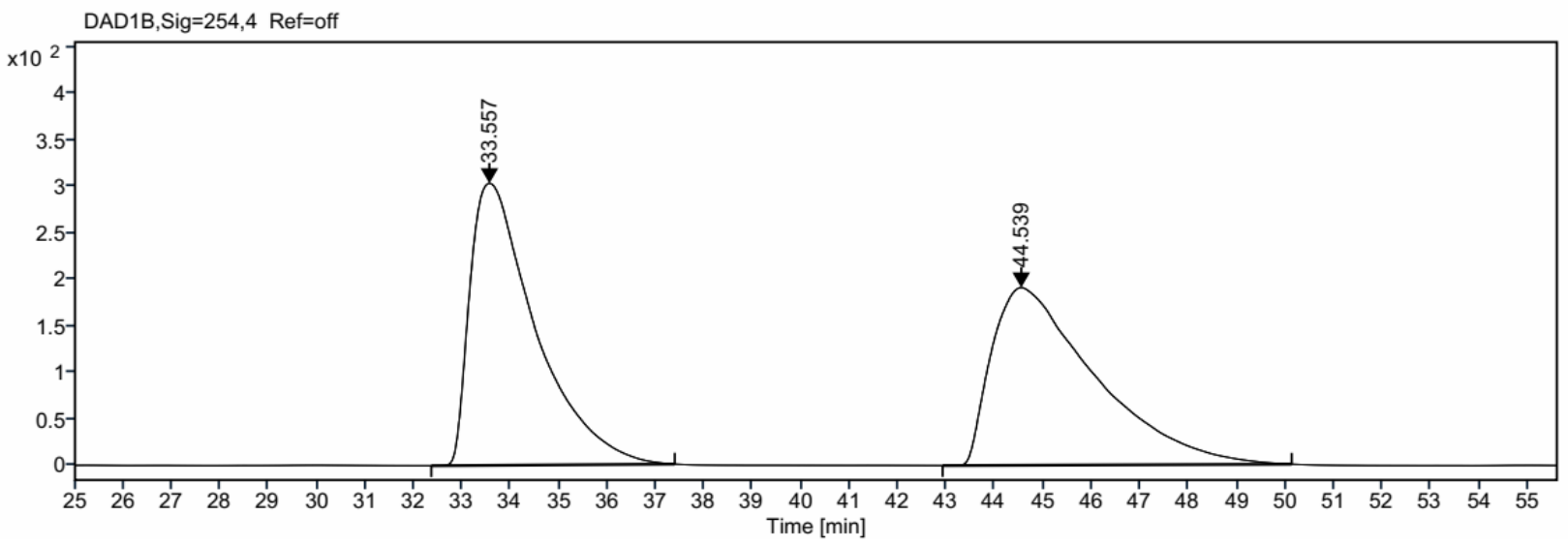

Signal: DAD1B,Sig=254,4 Ref=off

$\begin{array}{rrrrrr}\text { RT [min] } & \text { Type } & \text { Width [min] } & \text { Area } & \text { Height } & \text { Area\% } \\ 33.557 & \text { MM m } & 1.36 & 28406.55 & 302.84 & 50.14 \\ 44.539 & \text { MM m } & 2.18 & 28245.65 & 190.82 & 49.86 \\ & & \text { Sum } & 56652.20 & & \end{array}$

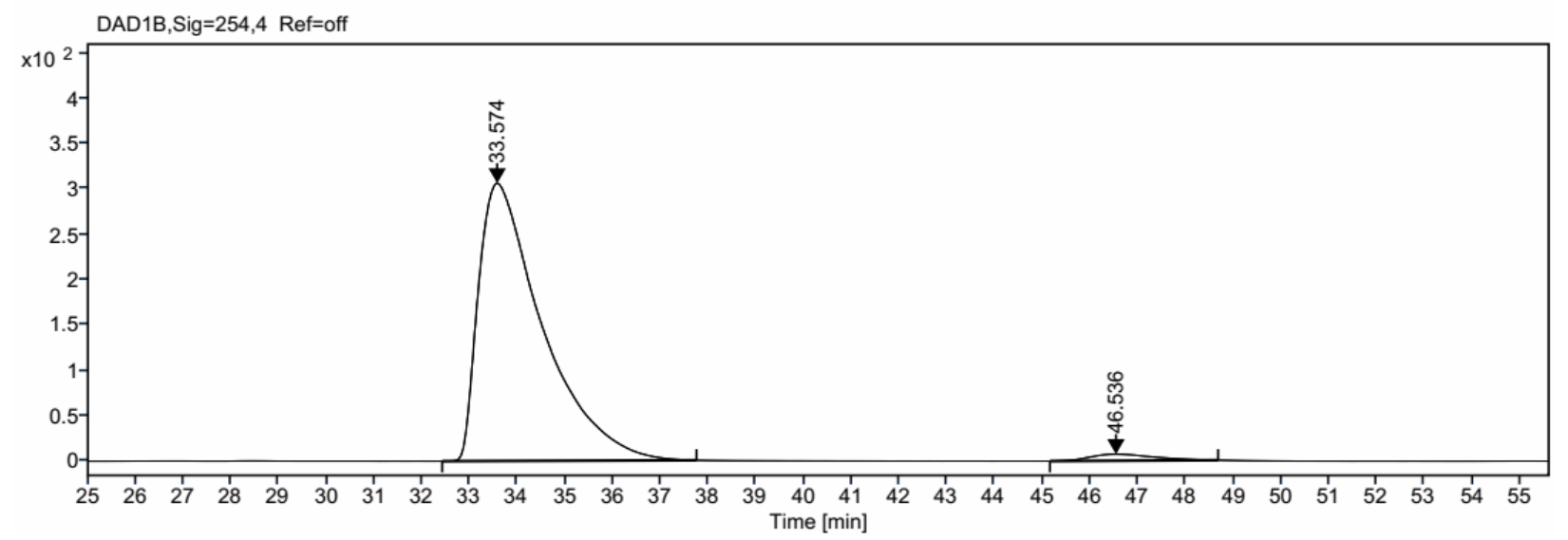

$\begin{array}{rrrrrr}\begin{array}{l}\text { Signal: } \\ \text { RT [min] }\end{array} & \text { Type } & \text { Width }[\mathrm{min}] & \text { Area } & \text { Height } & \text { Area\% } \\ 33.574 & \text { MM m } & 1.41 & 28423.61 & 306.51 & 97.68 \\ 46.536 & \text { MM m } & 1.17 & 675.56 & 7.26 & 2.32 \\ & & \text { Sum } & 29099.17 & & \end{array}$


<smiles>COC(=O)[C@H](O)[C@@H](O)c1ccc(C)cc1</smiles>

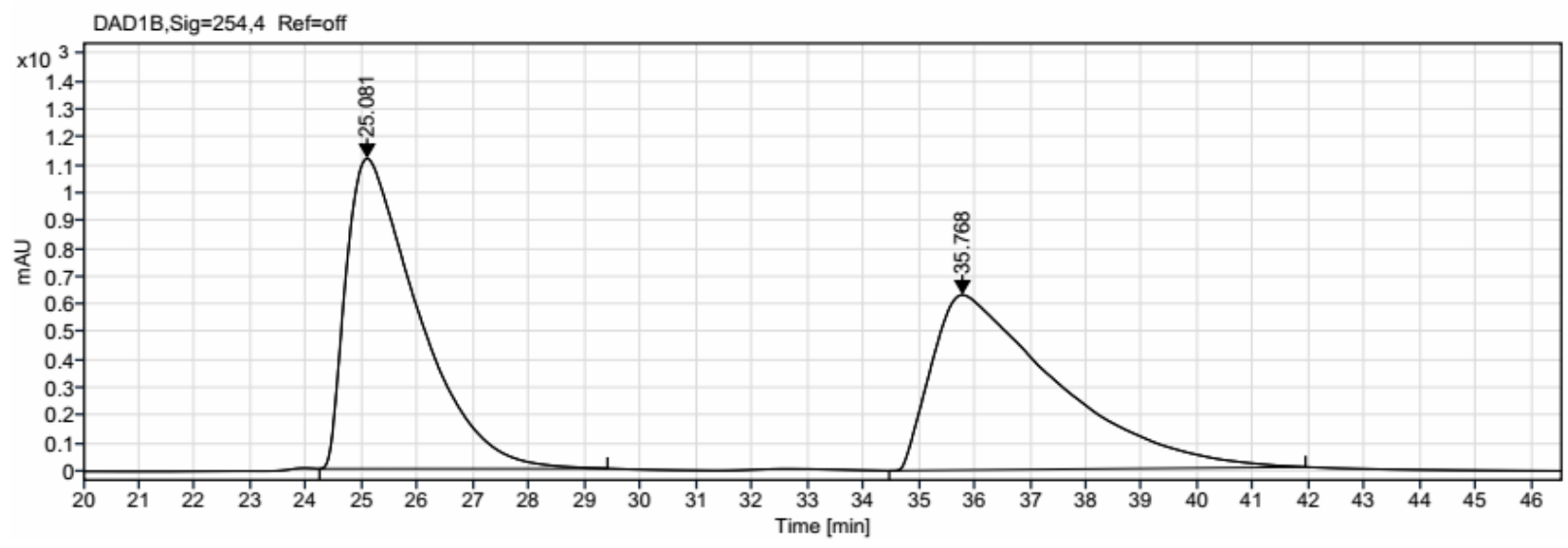

\begin{tabular}{rrrrrr}
\multicolumn{1}{l}{ Signal: } & \multicolumn{2}{c}{ DAD1B,Sig=254,4 Ref=off } & & & \\
RT [min] & Type & Width [min] & Area & Height & Area\% \\
25.081 & MM m & 1.29 & 100236.04 & 1114.27 & 50.64 \\
35.768 & MM m & 2.08 & 97707.75 & 629.34 & 49.36 \\
& & Sum & 197943.80 & &
\end{tabular}

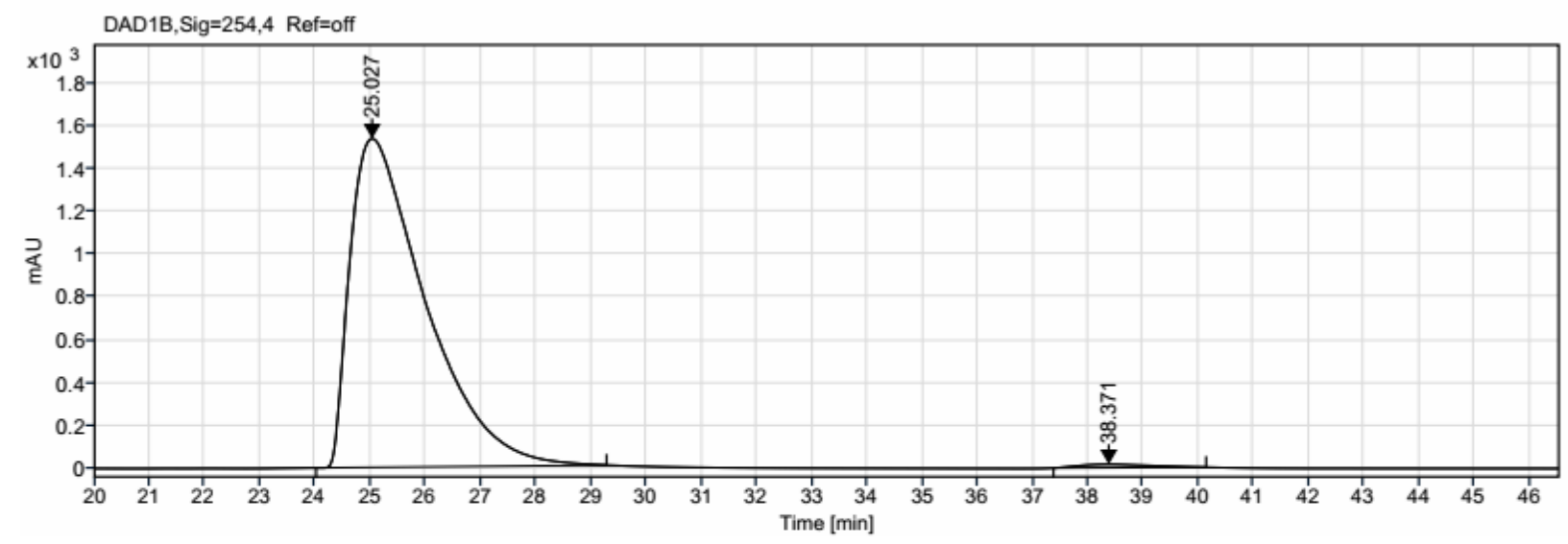

Signal: DAD1B,Sig $=254,4$ Ref $=$ off

$\begin{array}{rrrrrr}\text { RT [min] } & \text { Type } & \text { Width [min] } & \text { Area } & \text { Height } & \text { Area\% } \\ 25.027 & \text { MM m } & 1.36 & 145516.86 & 1535.24 & 99.03 \\ 38.371 & \text { MM m } & 1.15 & 1422.42 & 15.56 & 0.97\end{array}$



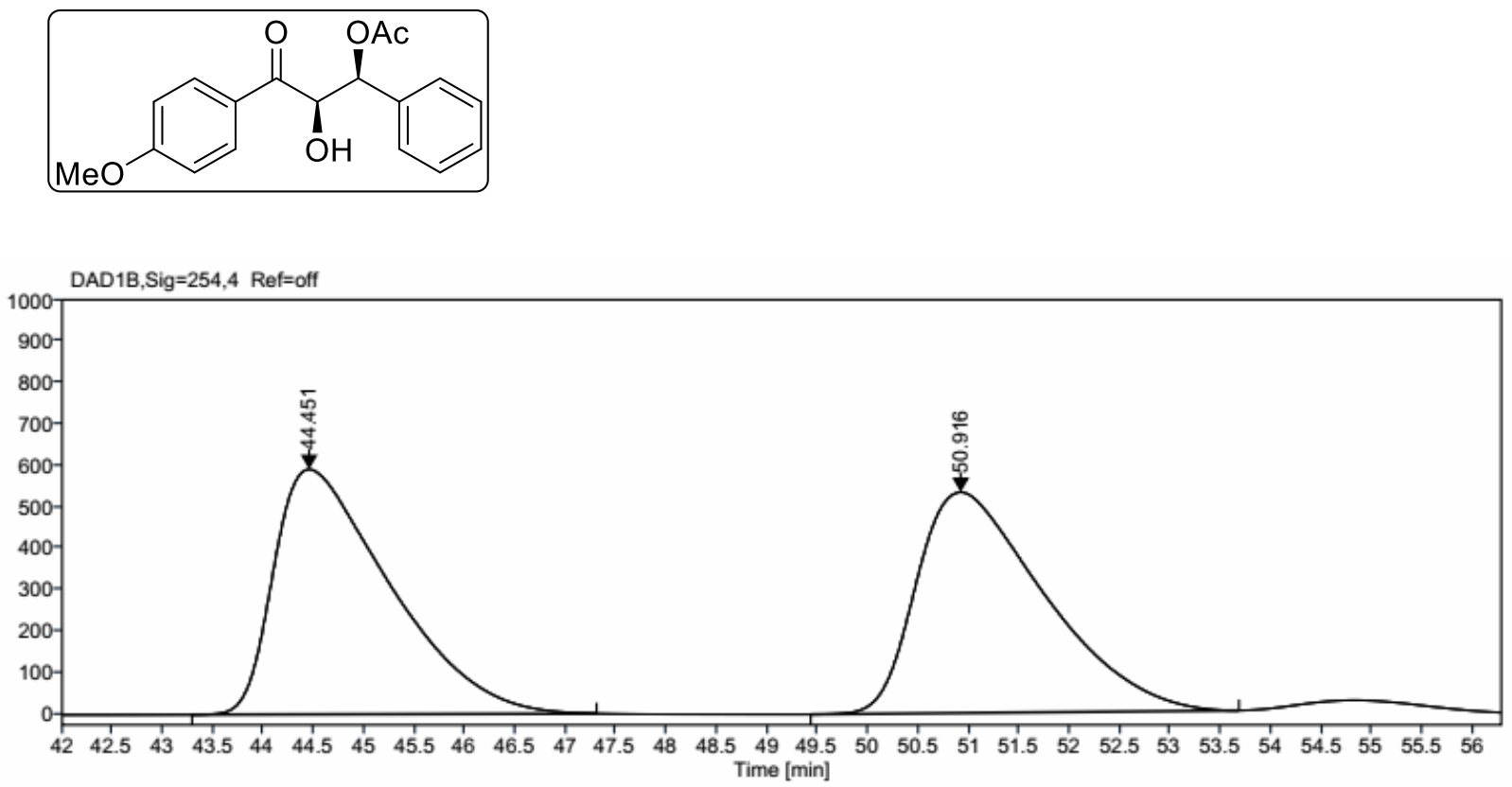

\begin{tabular}{|c|c|c|c|c|c|}
\hline Signal: & AD1B, & $=254,4$ Ref $=$ of & & & \\
\hline $\mathbf{R T}$ [min] & Type & Width [min] & Area & Height & Area\% \\
\hline 44.451 & $\mathrm{MM} \mathrm{m}$ & 1.17 & 47172.78 & 590.72 & 50.09 \\
\hline 50.916 & MM m & 1.35 & 46996.74 & 532.21 & 49.91 \\
\hline & & Sum & 94169.51 & & \\
\hline
\end{tabular}

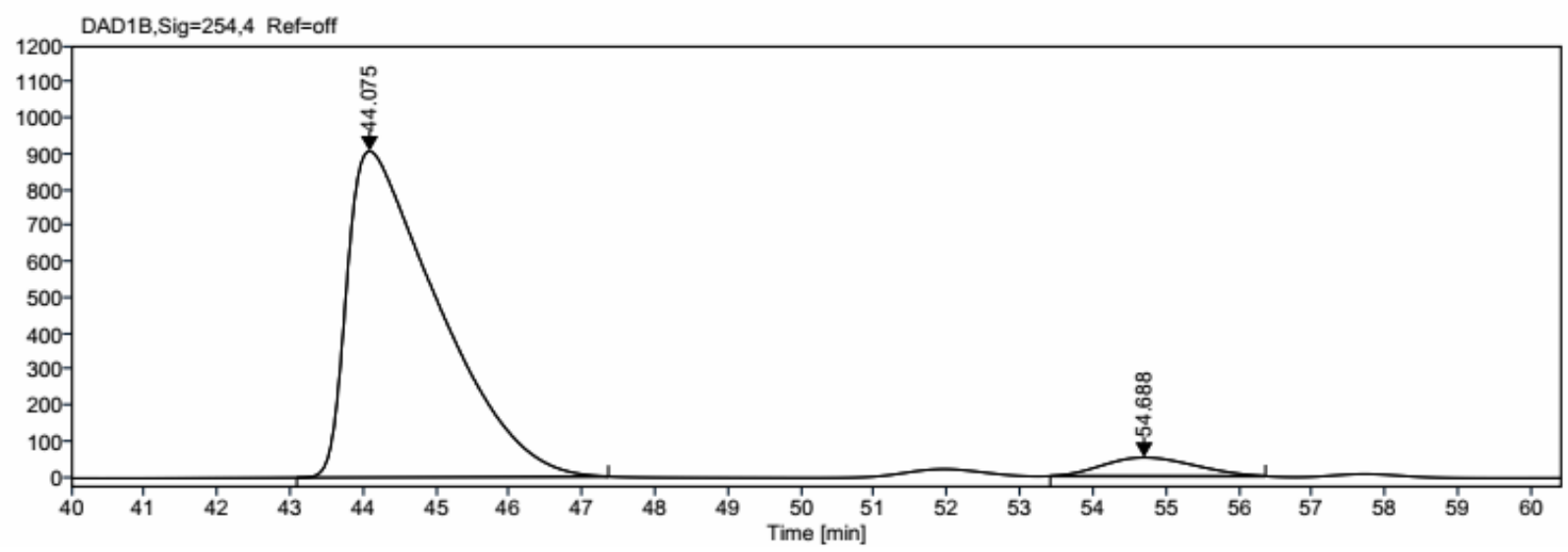

Signal: DAD1B,Sig $=254,4$ Ref $=$ off

$\begin{array}{rrrrrr}\text { RT [min] } & \text { Type } & \text { Width [min] } & \text { Area } & \text { Height } & \text { Area\% } \\ 44.075 & \text { MM m } & 1.24 & 78967.76 & 908.24 & 94.58 \\ 54.688 & \text { MM m } & 1.34 & 4522.52 & 51.88 & 5.42 \\ & & \text { Sum } & 83490.28 & & \end{array}$



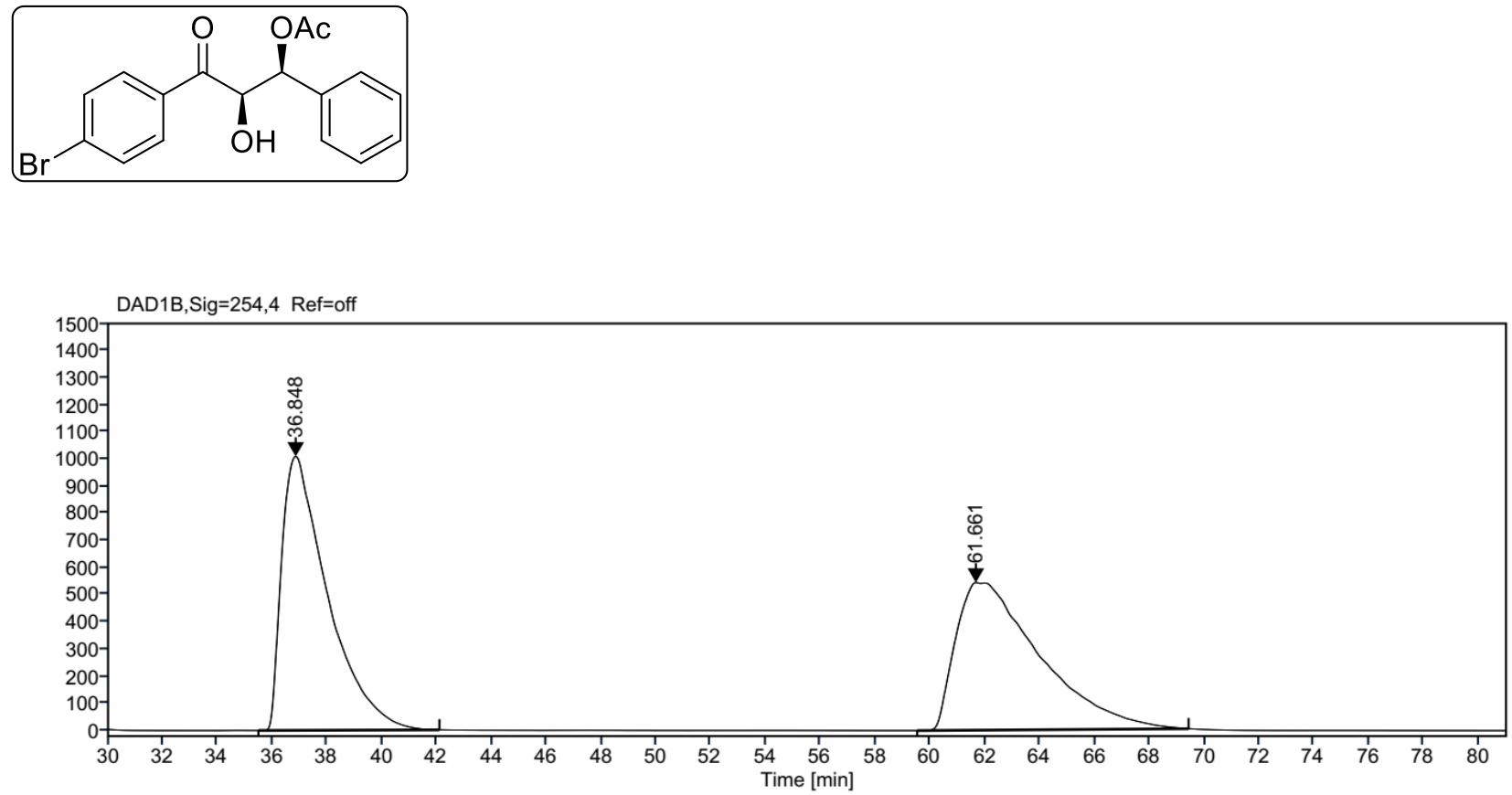

Signal: DAD1B,Sig $=254,4$ Ref=off

$\begin{array}{rrrrrr}\text { RT [min] } & \text { Type } & \text { Width [min] } & \text { Area } & \text { Height } & \text { Area\% } \\ 36.848 & \text { MM m } & 1.63 & 117249.81 & 1008.70 & 50.38 \\ 61.661 & \text { MM m } & 2.71 & 115480.64 & 543.72 & 49.62 \\ & & \text { Sum } & 232730.45 & & \end{array}$

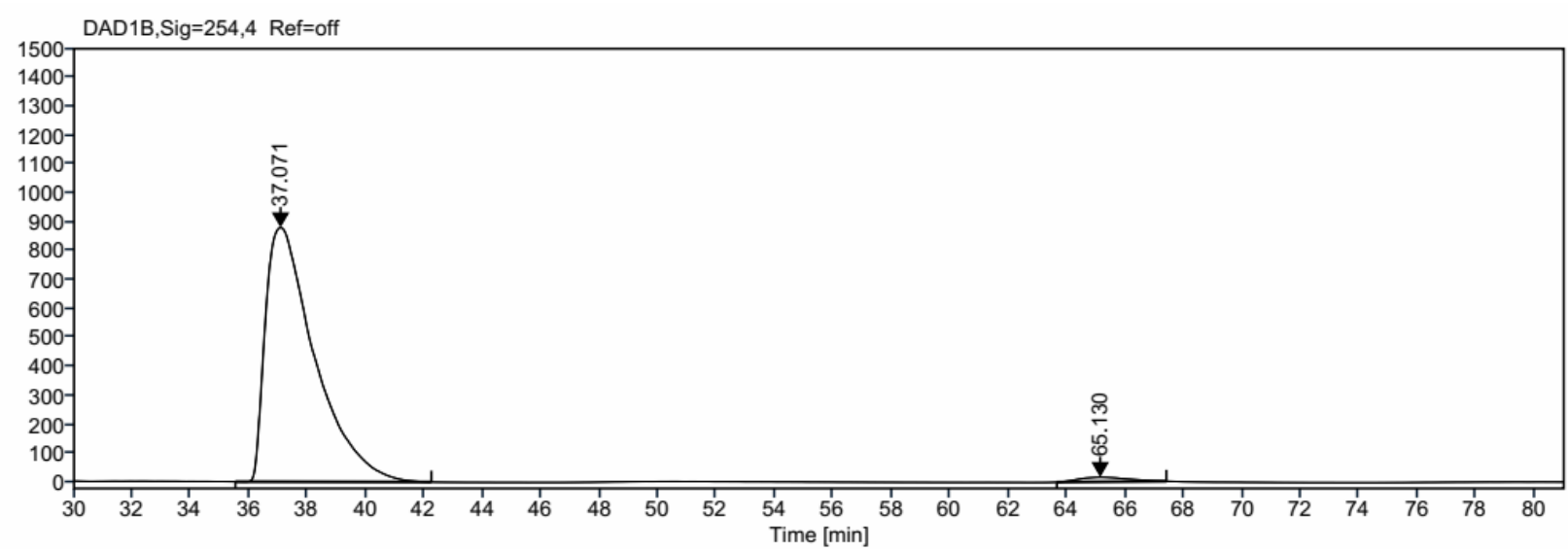

Signal: DAD1B,Sig $=254,4$ Ref $=$ off

$\begin{array}{rrrrrrr}\text { RT [min] } & \text { Type } & \text { Width [min] } & \text { Area } & \text { Height } & \text { Area\% } & \text { Name } \\ 37.071 & \text { MM m } & 1.73 & 102748.32 & 878.53 & 98.25 & \\ 65.130 & \text { MM m } & 1.41 & 1834.09 & 15.79 & 1.75 & \\ & & \text { Sum } & 104582.42 & & \end{array}$



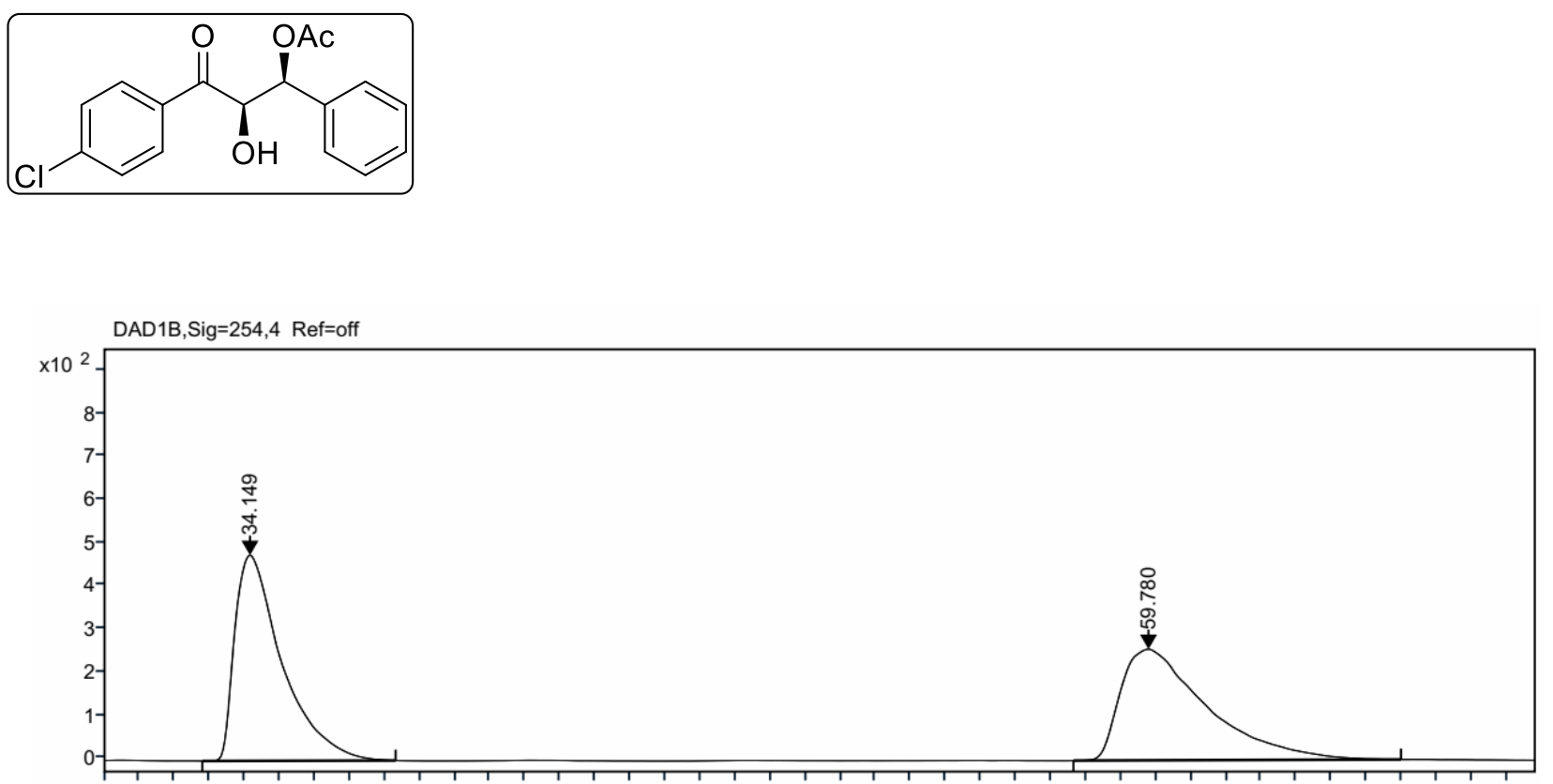

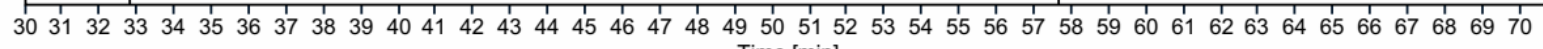
Time [min]

\begin{tabular}{rrrrrr}
\multicolumn{1}{l}{$\begin{array}{l}\text { Signal: } \\
\text { RT [min] }\end{array}$} & Type & Width [min] & Area & Height & Area\% \\
34.149 & MM m & 1.43 & 44740.45 & 477.63 & 50.51 \\
59.780 & MM m & 2.12 & 43833.22 & 258.17 & 49.49 \\
& & Sum & 88573.67 & &
\end{tabular}

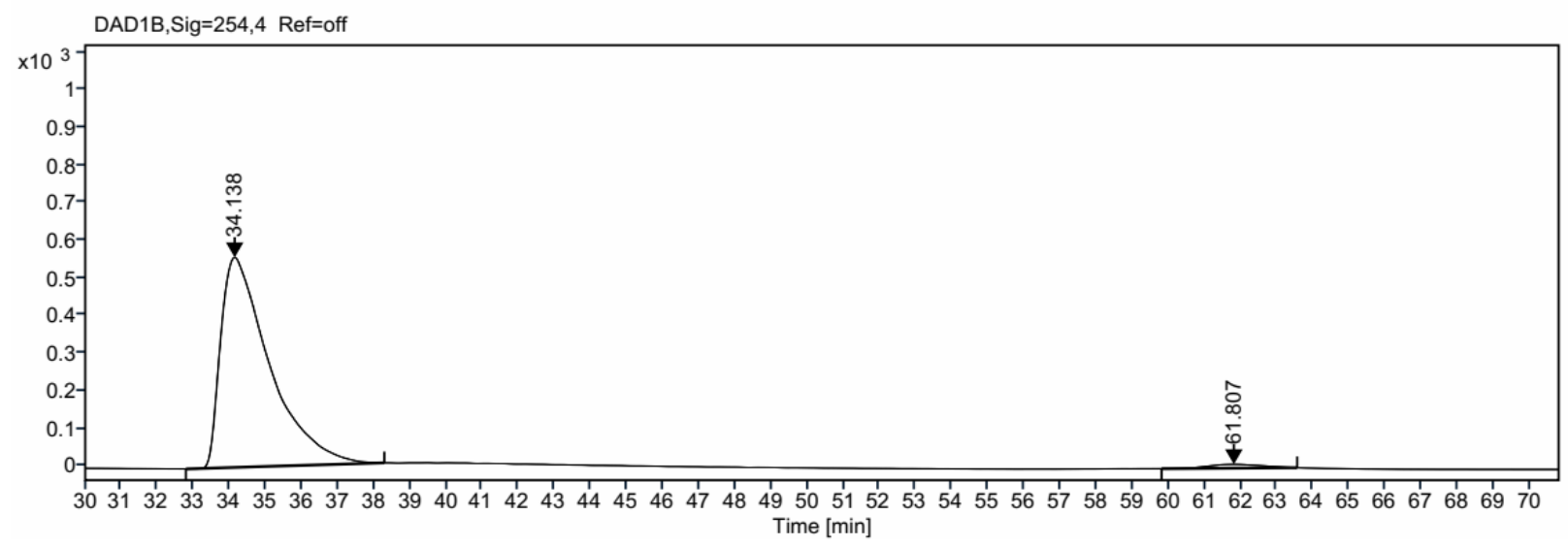

Signal: $\quad$ DAD1B,Sig=254,4 Ref=off

$\begin{array}{rrrrrrr}\text { RT [min] } & \text { Type } & \text { Width [min] } & \text { Area } & \text { Height } & \text { Area\% } & \text { Name } \\ 34.138 & \text { MM m } & 1.42 & 52200.33 & 557.72 & 98.07 \\ 61.807 & \text { MM m } & 1.21 & 1027.70 & 10.10 & 1.93 \\ & & \text { Sum } & 53228.03 & & \end{array}$



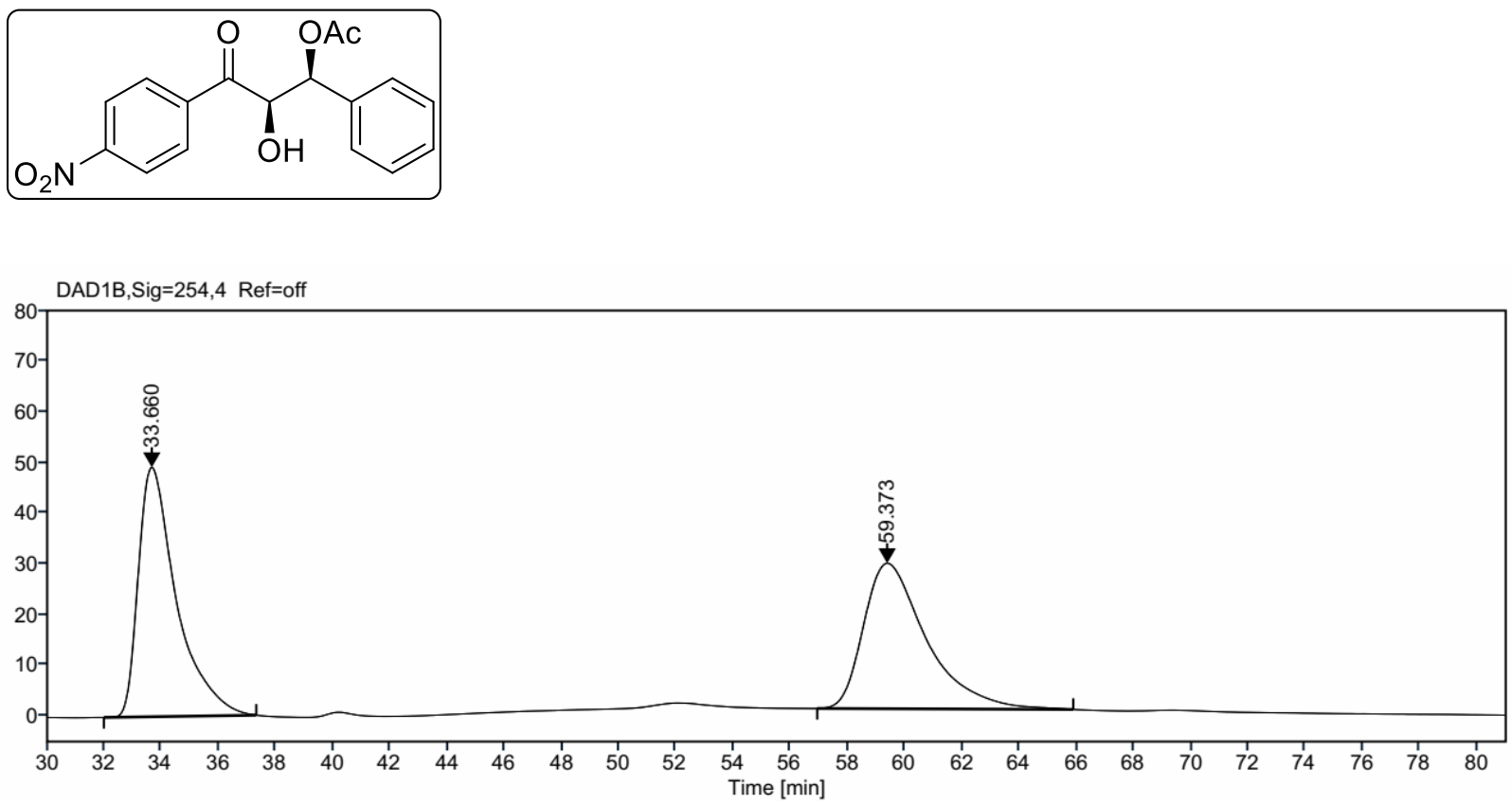

\begin{tabular}{rrrrrr} 
Signal: & \multicolumn{2}{c}{ DAD1B,Sig=254,4 Ref=off } & & & \\
RT [min] & Type & Width [min] & Area & Height & Area\% \\
33.660 & MM m & 1.36 & 4543.33 & 49.19 & 50.96 \\
59.373 & MM m & 2.03 & 4371.58 & 28.68 & 49.04 \\
& & Sum & 8914.91 & &
\end{tabular}

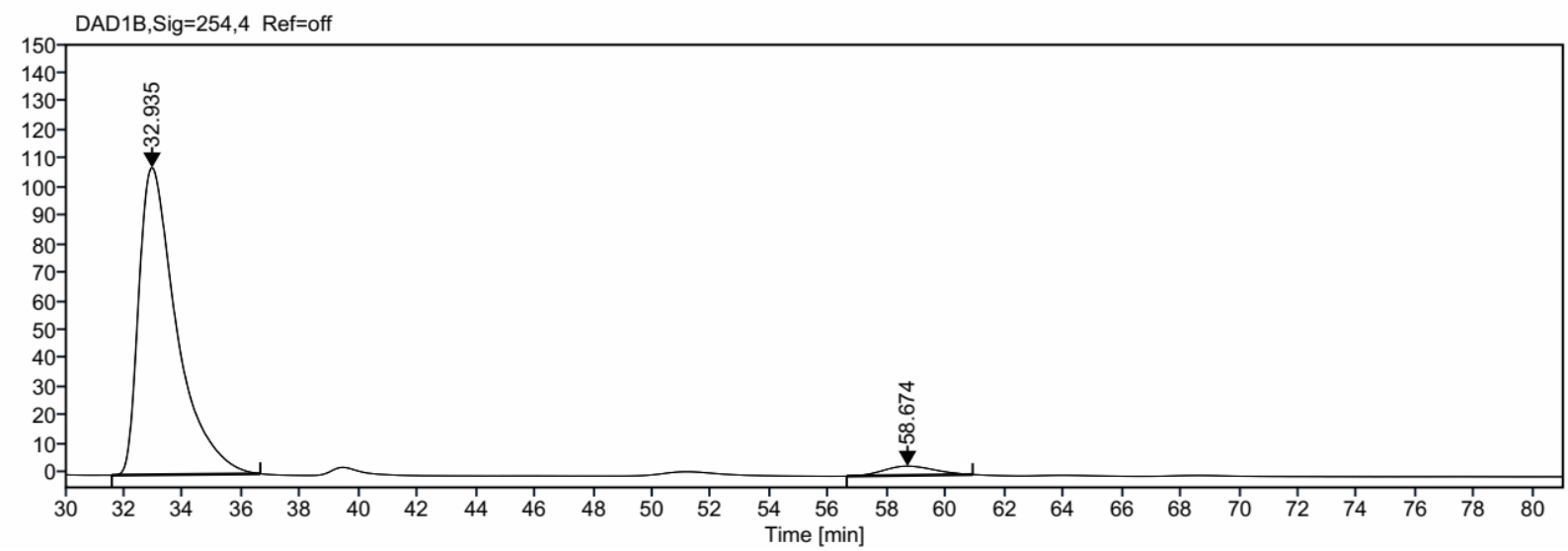

Signal: DAD1B,Sig=254,4 Ref=off

$\begin{array}{rrrrrrr}\text { RT [min] } & \text { Type } & \text { Width [min] } & \text { Area } & \text { Height } & \text { Area\% } & \text { Name } \\ 32.935 & \text { MM m } & 1.33 & 9618.28 & 107.53 & 96.15 \\ 58.674 & \text { MM m } & 1.40 & 385.32 & 3.23 & 3.85 \\ & & \text { Sum } & 10003.60 & & \end{array}$



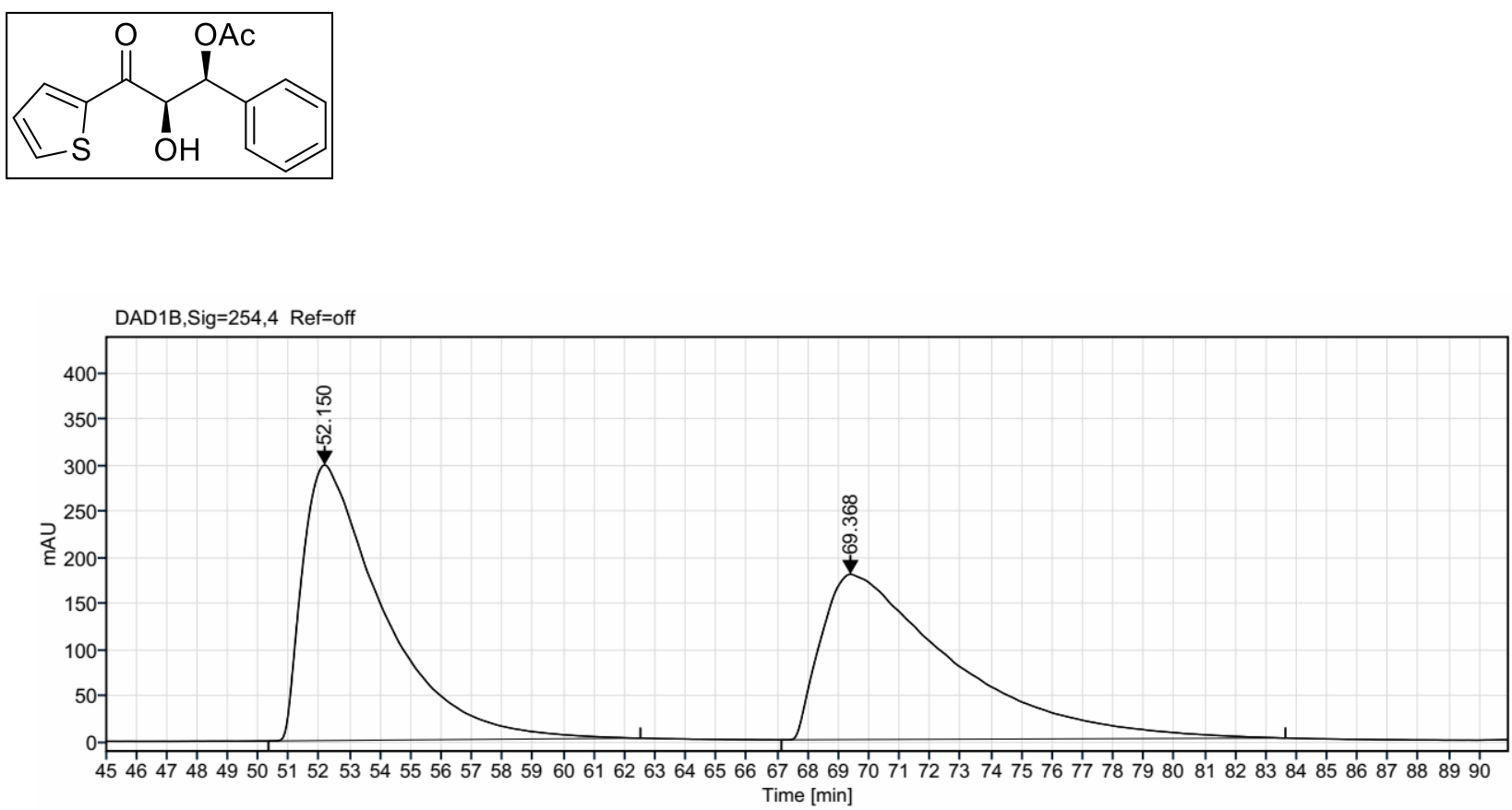

Signal: $\quad$ DAD1B,Sig=254,4 Ref=off

\begin{tabular}{|c|c|c|c|c|c|c|}
\hline RT [min] & Type & Width [min] & Area & Height & Area $\%$ & Name \\
\hline 52.150 & MM m & 2.52 & 54880.51 & 298.81 & 50.68 & \\
\hline \multirow[t]{2}{*}{69.368} & MM m & 3.74 & 53408.47 & 179.30 & 49.32 & \\
\hline & & Sum & 108288.98 & & & \\
\hline
\end{tabular}

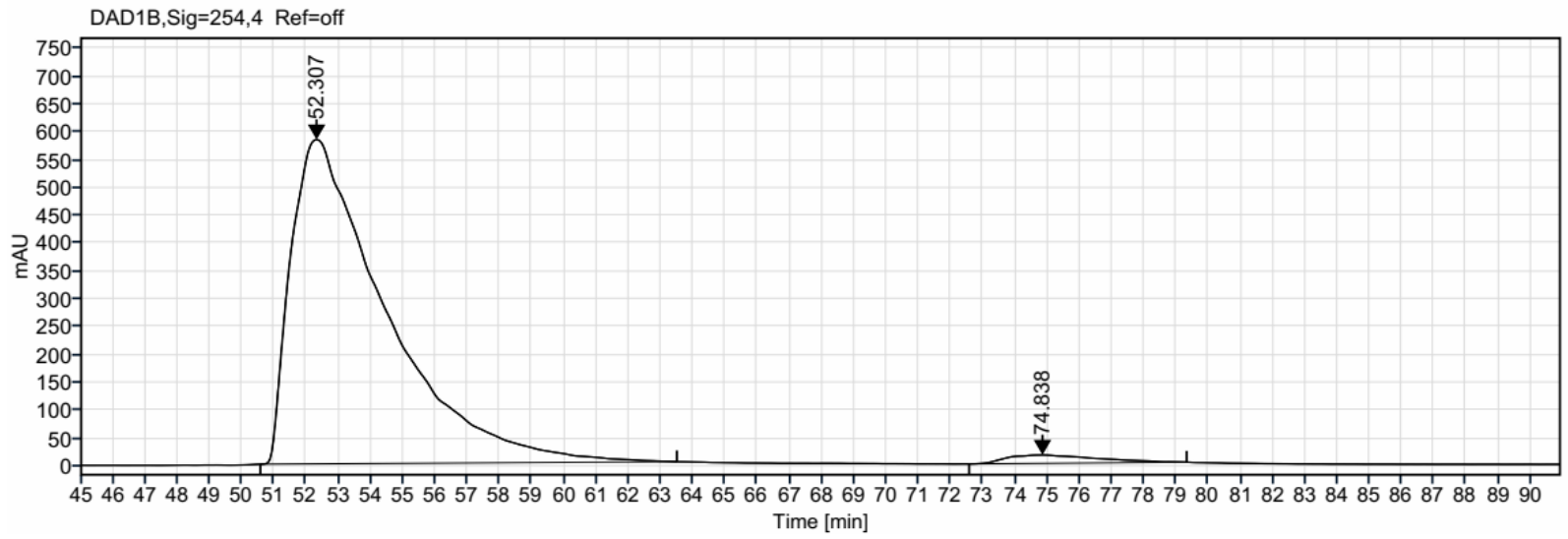

\begin{tabular}{lrrrrrr} 
Signal: & \multicolumn{2}{l}{ DAD1B,Sig=254,4 Ref=off } & & & \\
RT [min] & Type & Width [min] & Area & Height & Area\% & Name \\
52.307 & MM m & 2.62 & 119164.23 & 583.08 & 97.62 & \\
74.838 & MM m & 2.27 & 2901.15 & 15.03 & 2.38 \\
& & Sum & 122065.38 & &
\end{tabular}



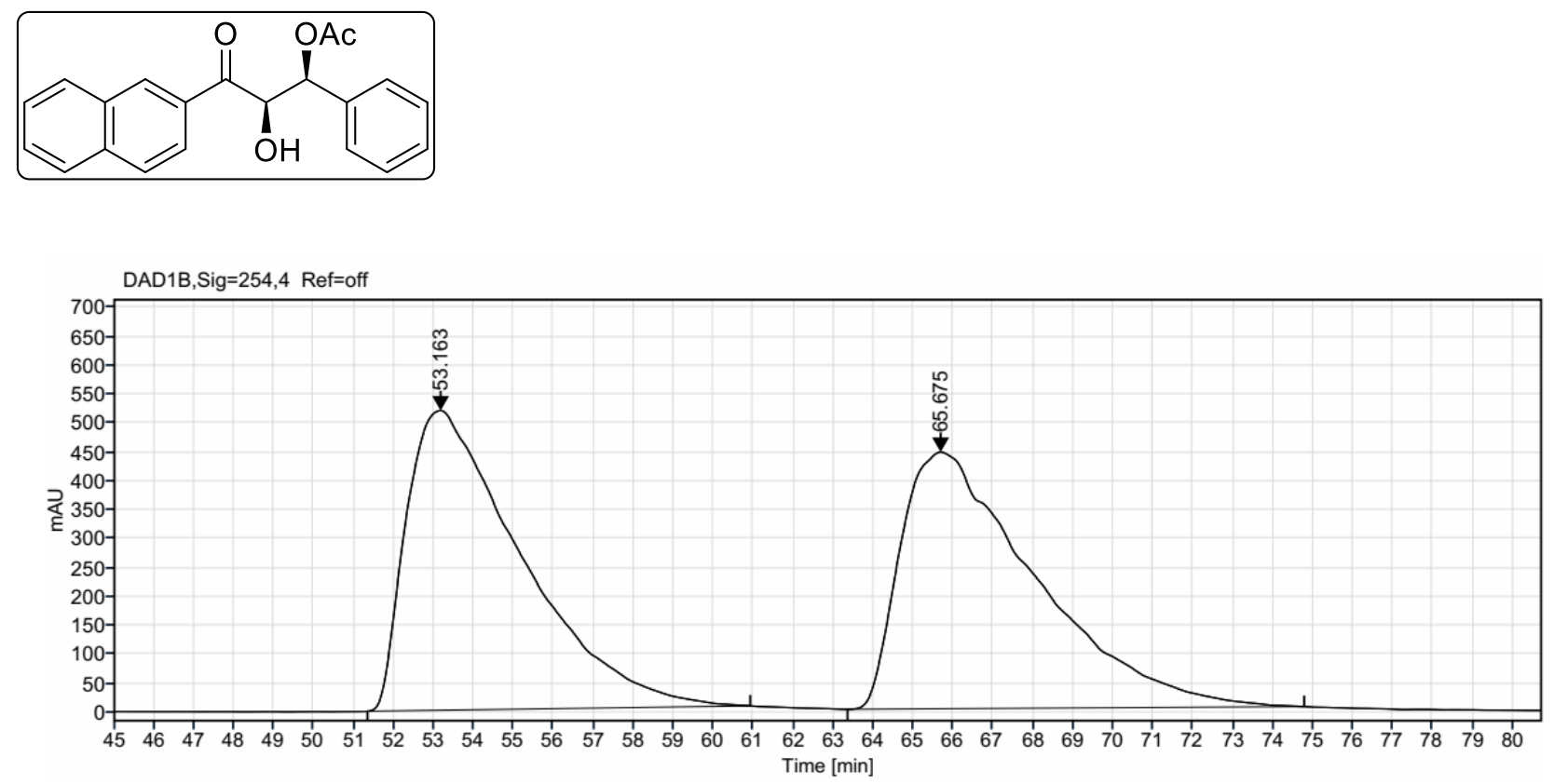

\begin{tabular}{|c|c|c|c|c|c|c|}
\hline Signal: & DAD1B,Si & $=254,4$ Ref $=$ off & & & & \\
\hline RT [min] & Type & Width [min] & Area & Height & Area\% & Name \\
\hline 53.163 & MM m & 2.61 & 103952.09 & 519.08 & 49.93 & \\
\hline 65.675 & $\mathrm{MM} \mathrm{m}$ & 3.00 & 104231.85 & 444.50 & 50.07 & \\
\hline & & Sum & 208183.93 & & & \\
\hline
\end{tabular}

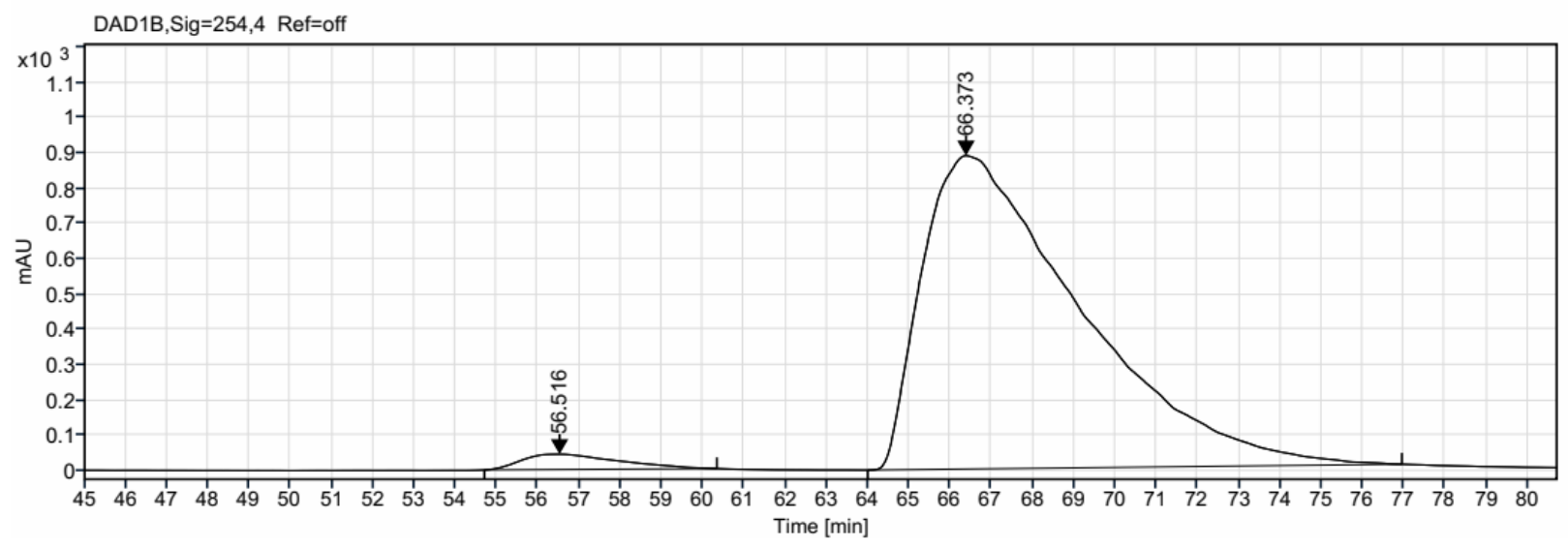

\begin{tabular}{rrrrrrr}
\multicolumn{1}{l}{ Signal: } & \multicolumn{2}{c}{ DAD1B,Sig=254,4 Ref=off } & & & & Name \\
RT [min] & Type & Width [min] & Area & Height & Area\% & \\
56.516 & MM m & 1.96 & 7034.02 & 43.21 & 2.88 & \\
66.373 & MM m & 3.23 & 237102.32 & 888.04 & 97.12 & \\
& & Sum & 244136.34 & & &
\end{tabular}



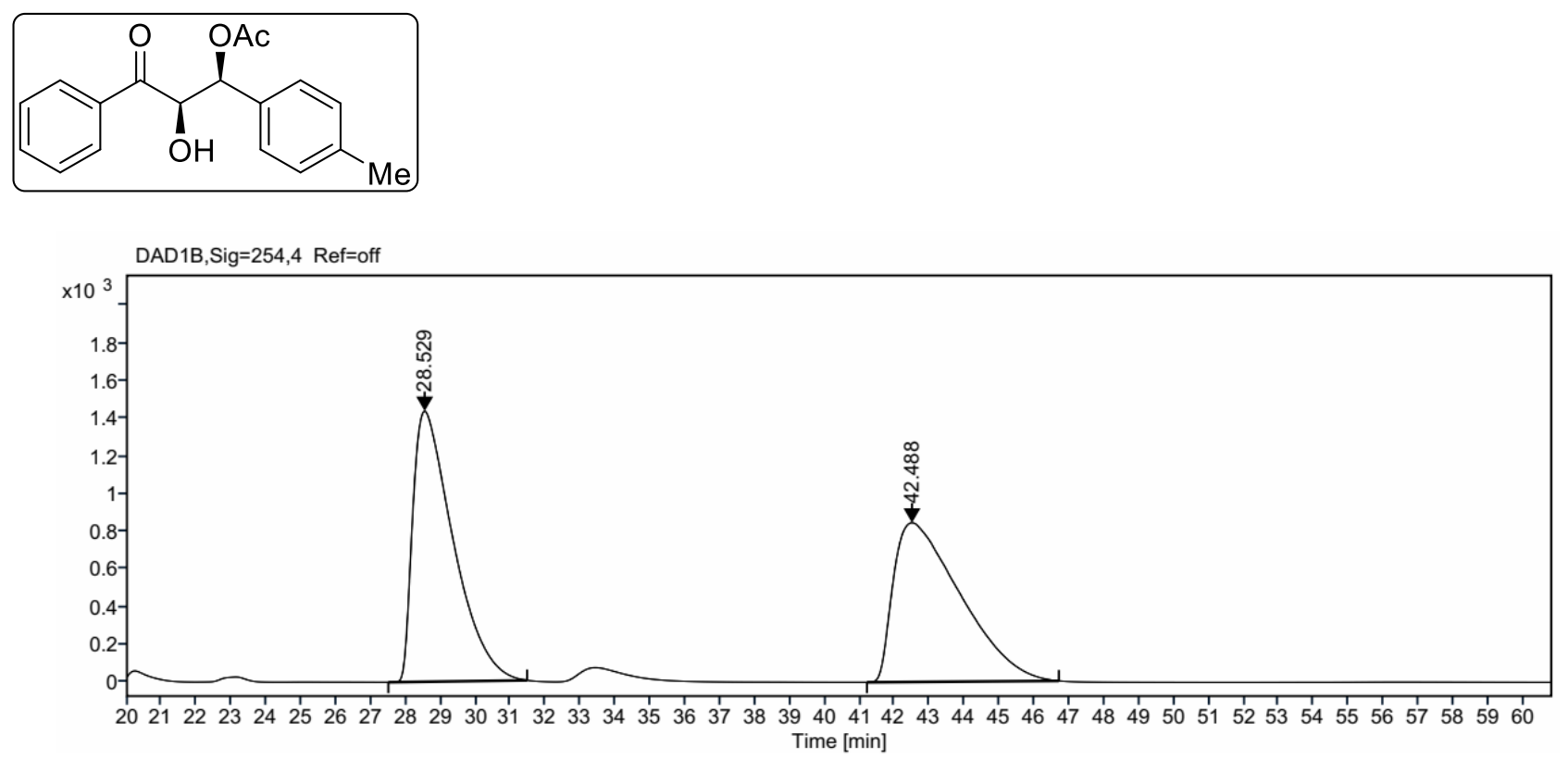

Signal: DAD1B, Sig $=254,4$ Ref=off

$\begin{array}{rrrrrr}\text { RT [min] } & \text { Type } & \text { Width [min] } & \text { Area } & \text { Height } & \text { Area\% } \\ 28.529 & \text { MM m } & 1.22 & 115404.72 & 1436.67 & 50.58 \\ 42.488 & \text { MM m } & 1.83 & 112778.01 & 845.00 & 49.42 \\ & & \text { Sum } & 228182.73 & & \end{array}$

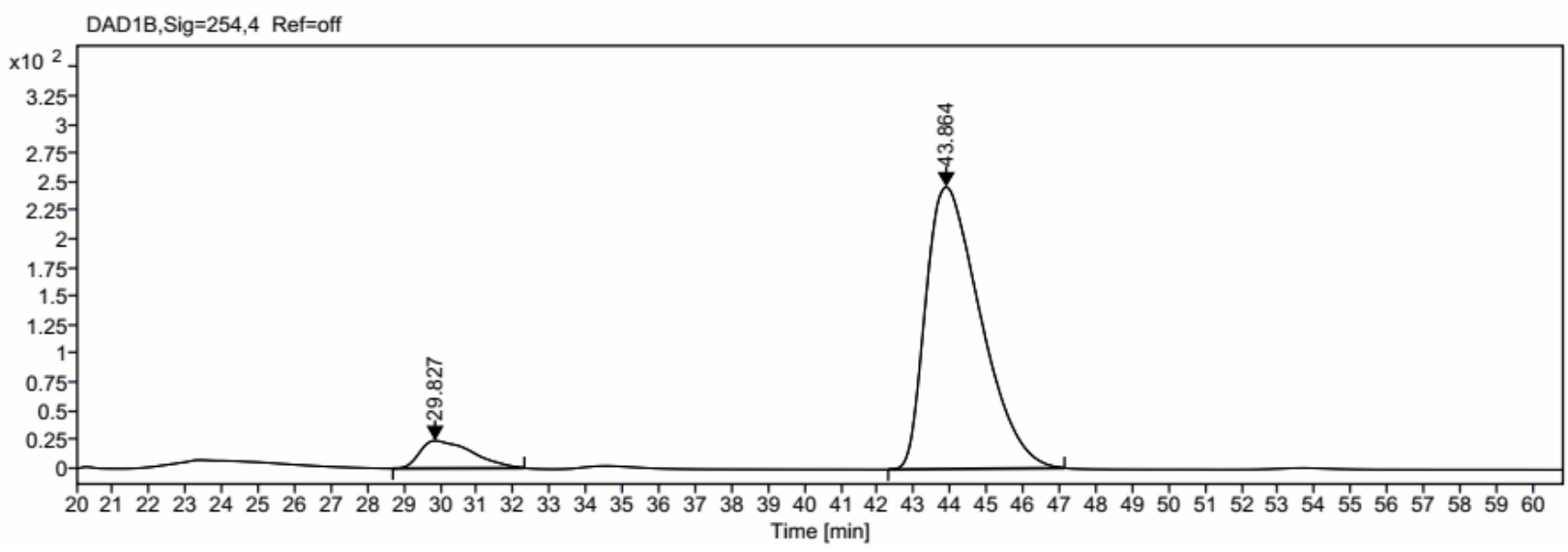

\begin{tabular}{rrrrrrr} 
Signal: & \multicolumn{2}{c}{ DAD1B,Sig=254,4 Ref=off } & & & & Name \\
RT [min] & Type & Width [min] & Area & Height & Area\% & \\
29.827 & MM m & 1.35 & 2389.89 & 24.08 & 8.49 & \\
43.864 & MM m & 1.62 & 25772.04 & 246.19 & 91.51 \\
& & Sum & 28161.93 & & &
\end{tabular}



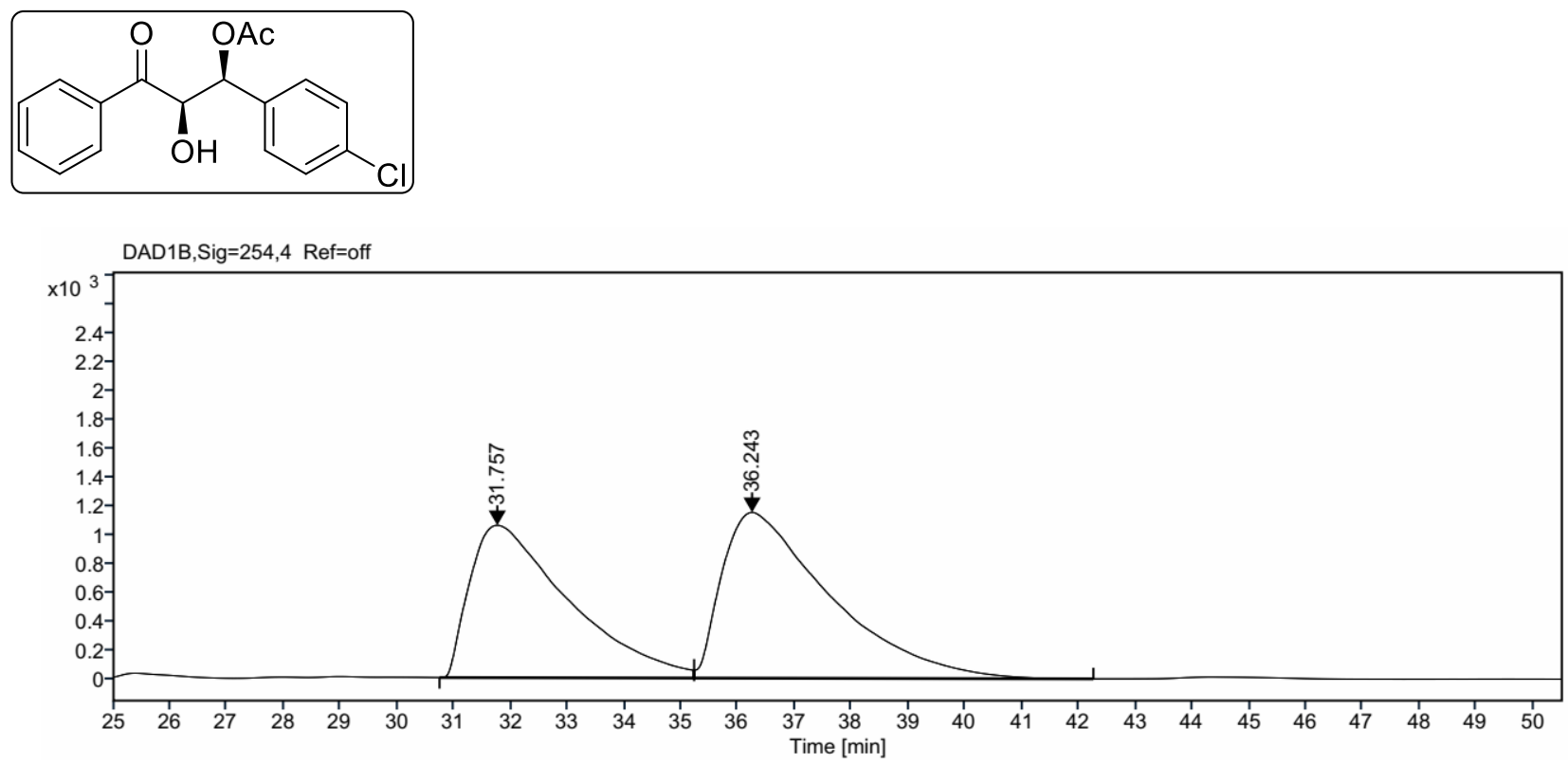

Signal: DAD1B,Sig $=254,4$ Ref=off

$\begin{array}{rrrrrr}\text { RT [min] } & \text { Type } & \text { Width [min] } & \text { Area } & \text { Height } & \text { Area\% } \\ 31.757 & \text { BV } & 4.48 & 127318.74 & 1055.40 & 45.65 \\ 36.243 & \text { VB } & 7.03 & 151571.00 & 1148.91 & 54.35 \\ & & \text { Sum } & 278889.73 & & \end{array}$

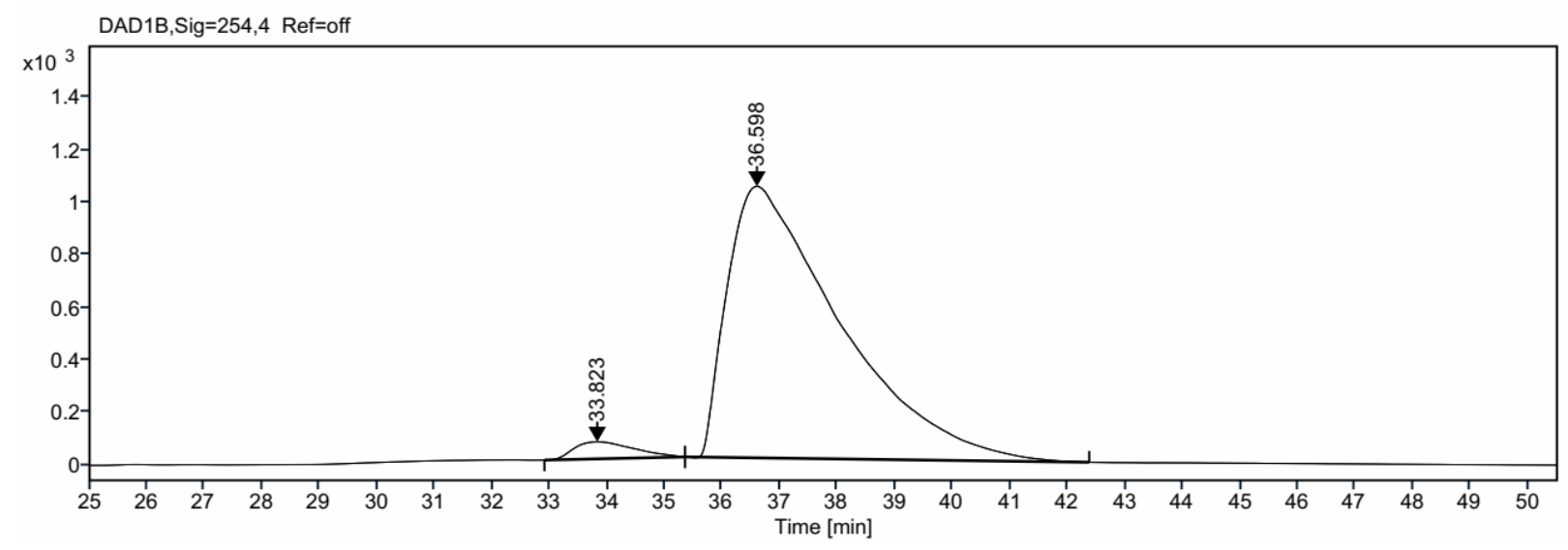

Signal: $\quad$ DAD1B,, ig $=254,4$ Ref $=$ off

$\begin{array}{rrrrrr}\text { RT [min] } & \text { Type } & \text { Width [min] } & \text { Area } & \text { Height } & \text { Area\% } \\ 33.823 & \text { MM m } & 1.08 & 4658.68 & 64.98 & 3.20 \\ 36.598 & \text { MM m } & 1.89 & 140777.01 & 1036.33 & 96.80 \\ & & \text { Sum } & 145435.69 & & \end{array}$



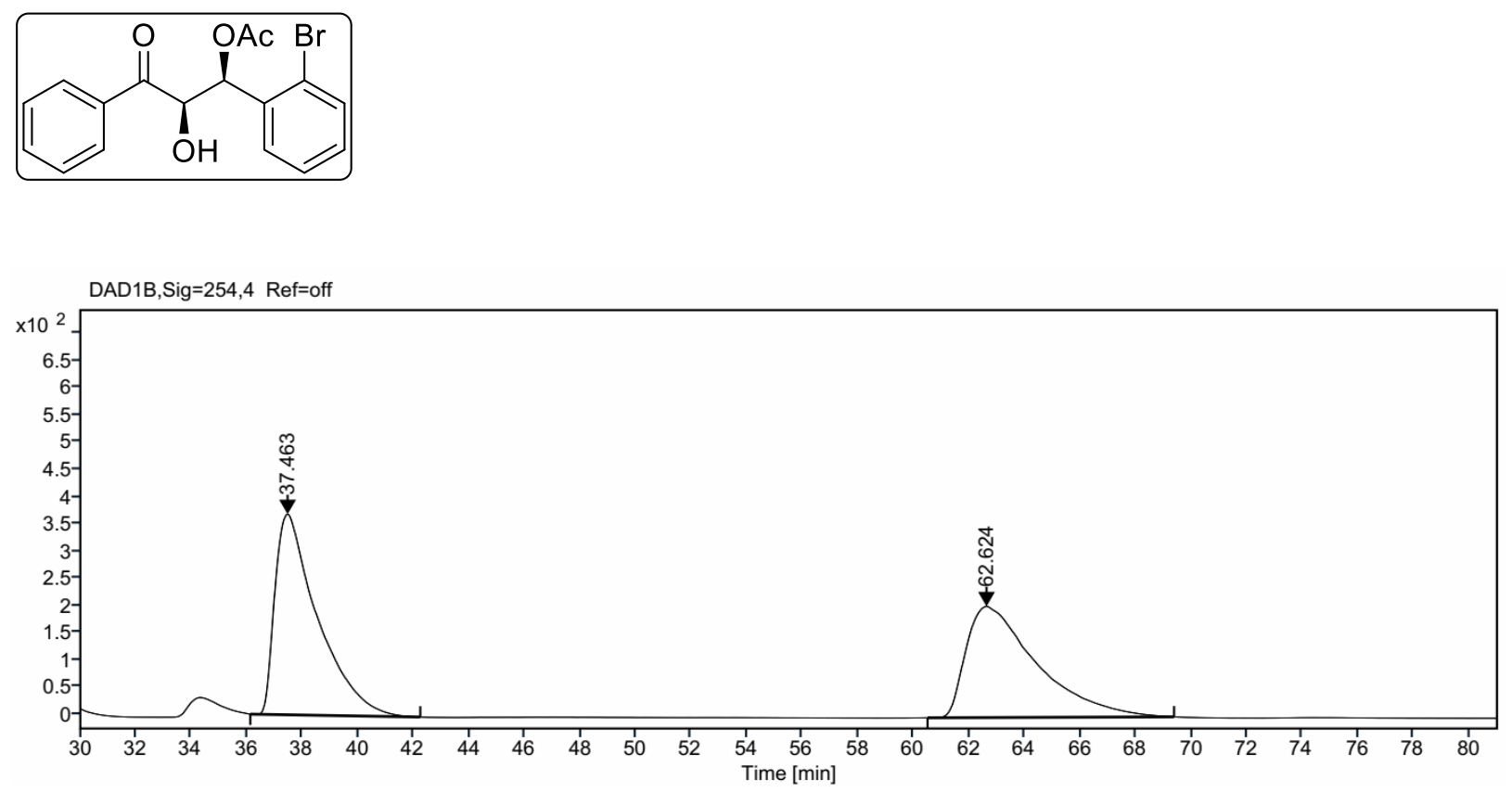

Signal: $\quad$ DAD1B,Sig=254,4 Ref=off

$\begin{array}{rrrrrr}\text { RT [min] } & \text { Type } & \text { Width [min] } & \text { Area } & \text { Height } & \text { Area\% } \\ 37.463 & \text { MM m } & 1.53 & 39272.64 & 368.76 & 52.23 \\ 62.624 & \text { MM m } & 2.34 & 35917.33 & 204.36 & 47.77 \\ & & \text { Sum } & 75189.97 & & \end{array}$

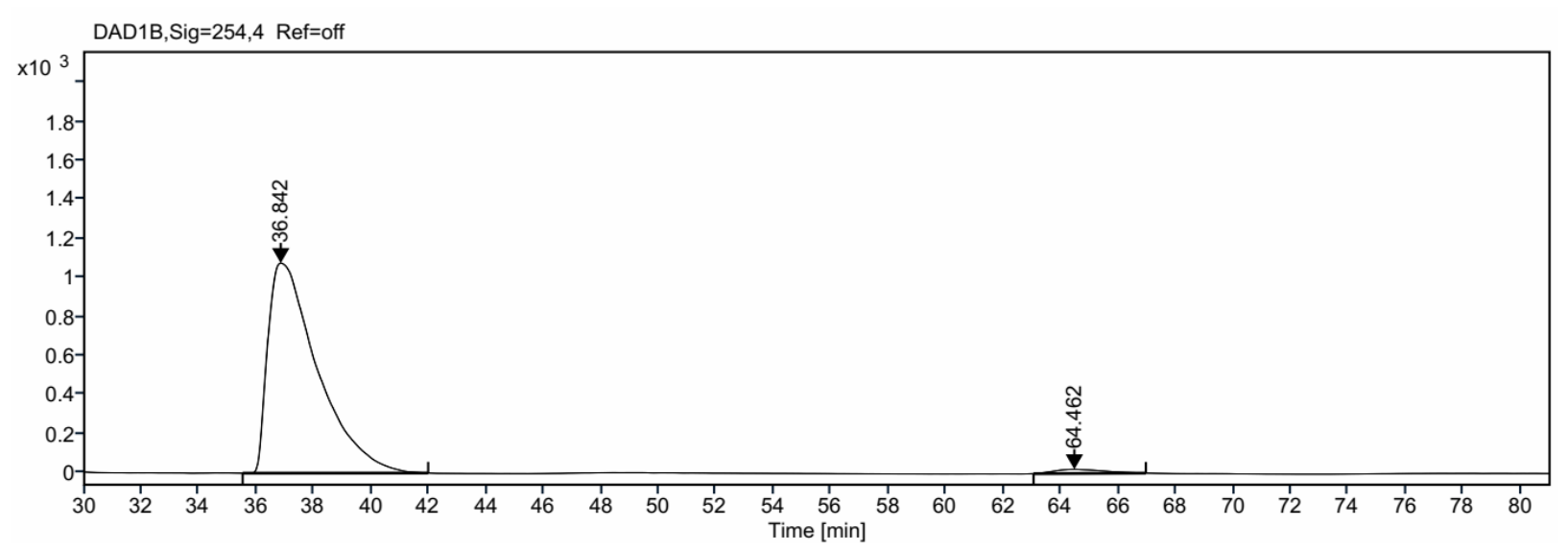

\begin{tabular}{rrrrrr} 
Signal: & \multicolumn{2}{c}{ DAD1B,Sig=254,4 Ref=off } & & & \\
RT [min] & Type & Width [min] & Area & Height & Area\% \\
36.842 & MM m & 1.73 & 128924.31 & 1076.70 & 98.09 \\
64.462 & MM m & 1.59 & 2508.54 & 21.23 & 1.91 \\
& & Sum & 131432.85 & &
\end{tabular}



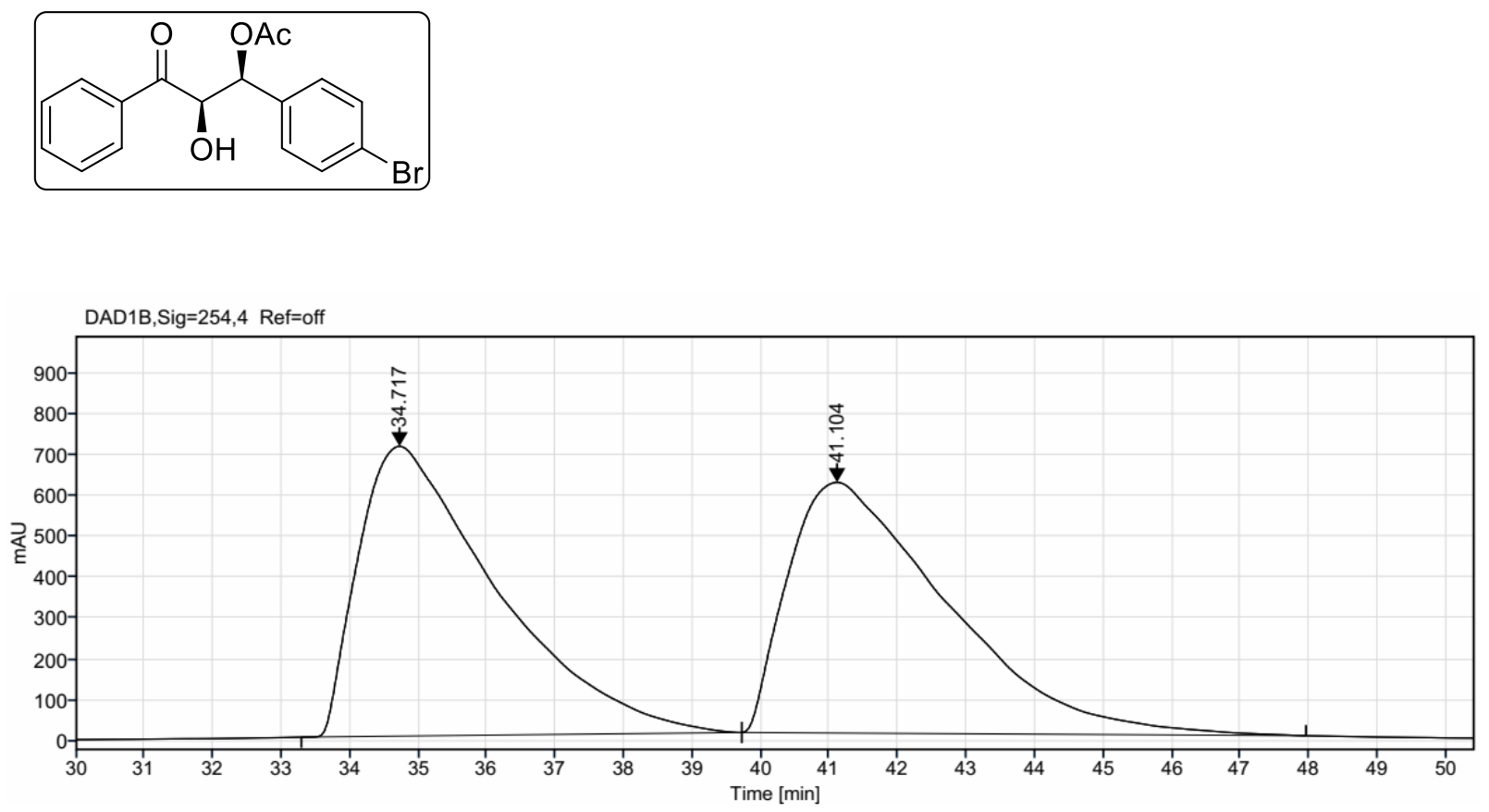

\begin{tabular}{|c|c|c|c|c|c|c|}
\hline Signal: & $\mathrm{AD} 1 \mathrm{~B}, \mathrm{~S}$ & $=254,4$ Ref $=$ off & & & & \\
\hline RT [min] & Type & Width [min] & Area & Height & Area\% & Name \\
\hline 34.717 & MM m & 1.90 & 99194.00 & 708.61 & 50.03 & \\
\hline \multirow[t]{2}{*}{41.104} & $\mathrm{MM} \mathrm{m}$ & 2.16 & 99065.71 & 612.90 & 49.97 & \\
\hline & & Sum & 198259.71 & & & \\
\hline
\end{tabular}

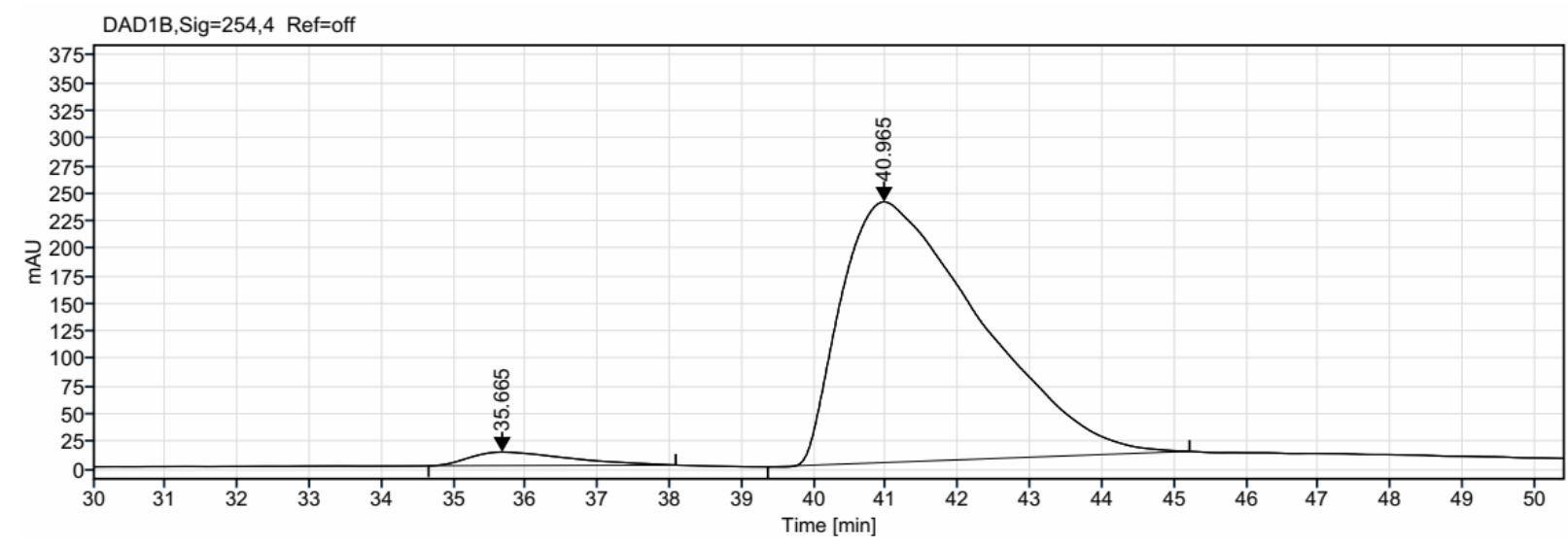

\begin{tabular}{rrrrrrr}
\multicolumn{1}{l}{$\begin{array}{l}\text { Signal: } \\
\text { RT [min] }\end{array}$} & Type & Width $[$ min] & Area & Height & Area\% & Name \\
35.665 & MM m & 1.30 & 1172.64 & 12.33 & 3.58 & \\
40.965 & MM m & 1.86 & 31608.13 & 236.36 & 96.42 \\
& & Sum & 32780.78 & &
\end{tabular}



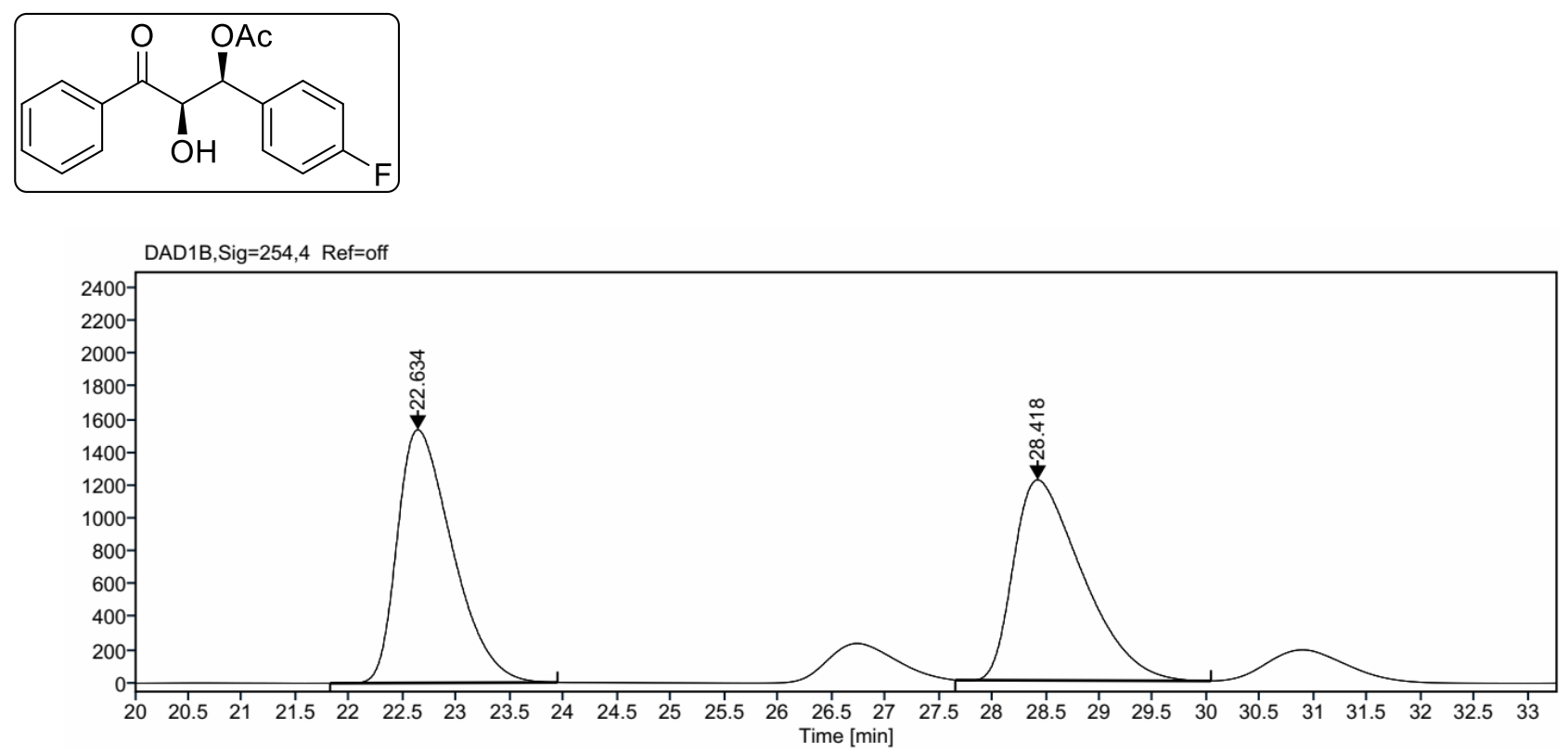

\begin{tabular}{rrrrrr}
\multicolumn{1}{l}{$\begin{array}{l}\text { Signal: } \\
\text { RT [min] }\end{array}$} & TAD1B,Sig=254,4 Ref=off & Width [min] & Area & Height & Area\% \\
22.634 & MM m & 0.55 & 55011.17 & 1539.17 & 50.22 \\
28.418 & MM m & 0.68 & 54524.11 & 1219.04 & 49.78 \\
& & Sum & 109535.28 & &
\end{tabular}

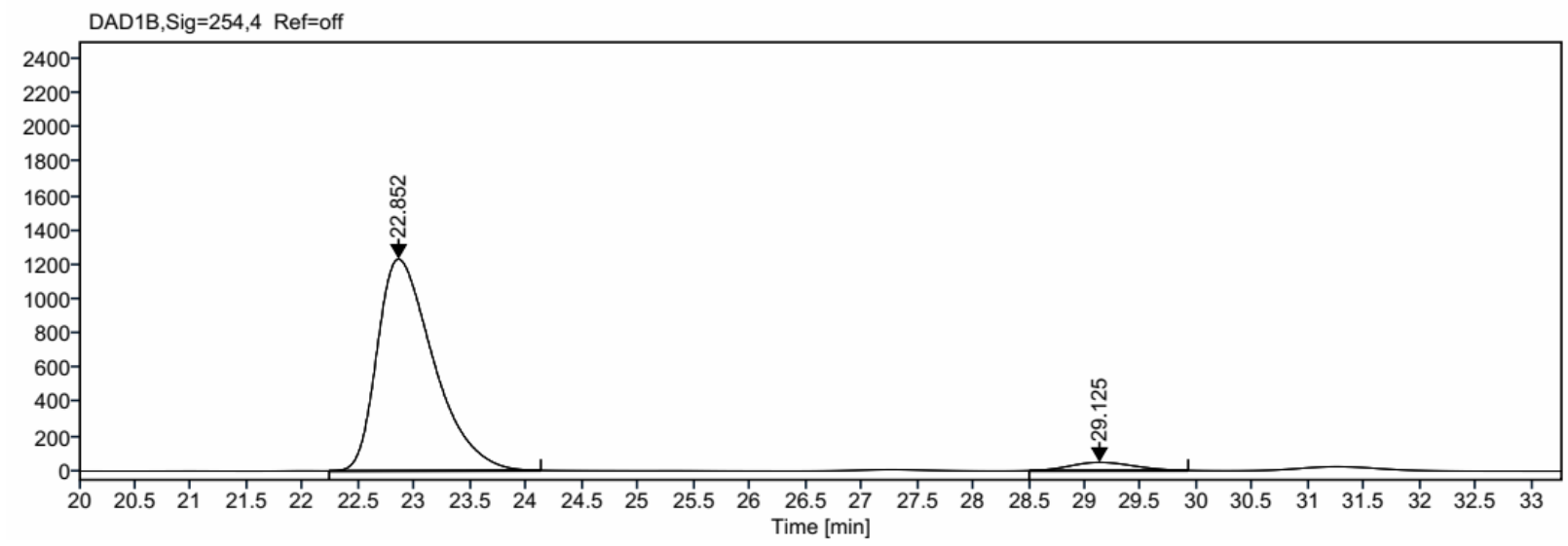

\begin{tabular}{rrrrrr}
\multicolumn{1}{l}{$\begin{array}{l}\text { Signal: } \\
\text { RT [min] }\end{array}$} & TyD1B,Sig=254,4 Ref=off & Width [min] & Area & Height & Area\% \\
22.852 & MM m & 0.54 & 43239.70 & 1233.84 & 95.79 \\
29.125 & MM m & 0.62 & 1900.18 & 48.22 & 4.21 \\
& & Sum & 45139.87 & &
\end{tabular}



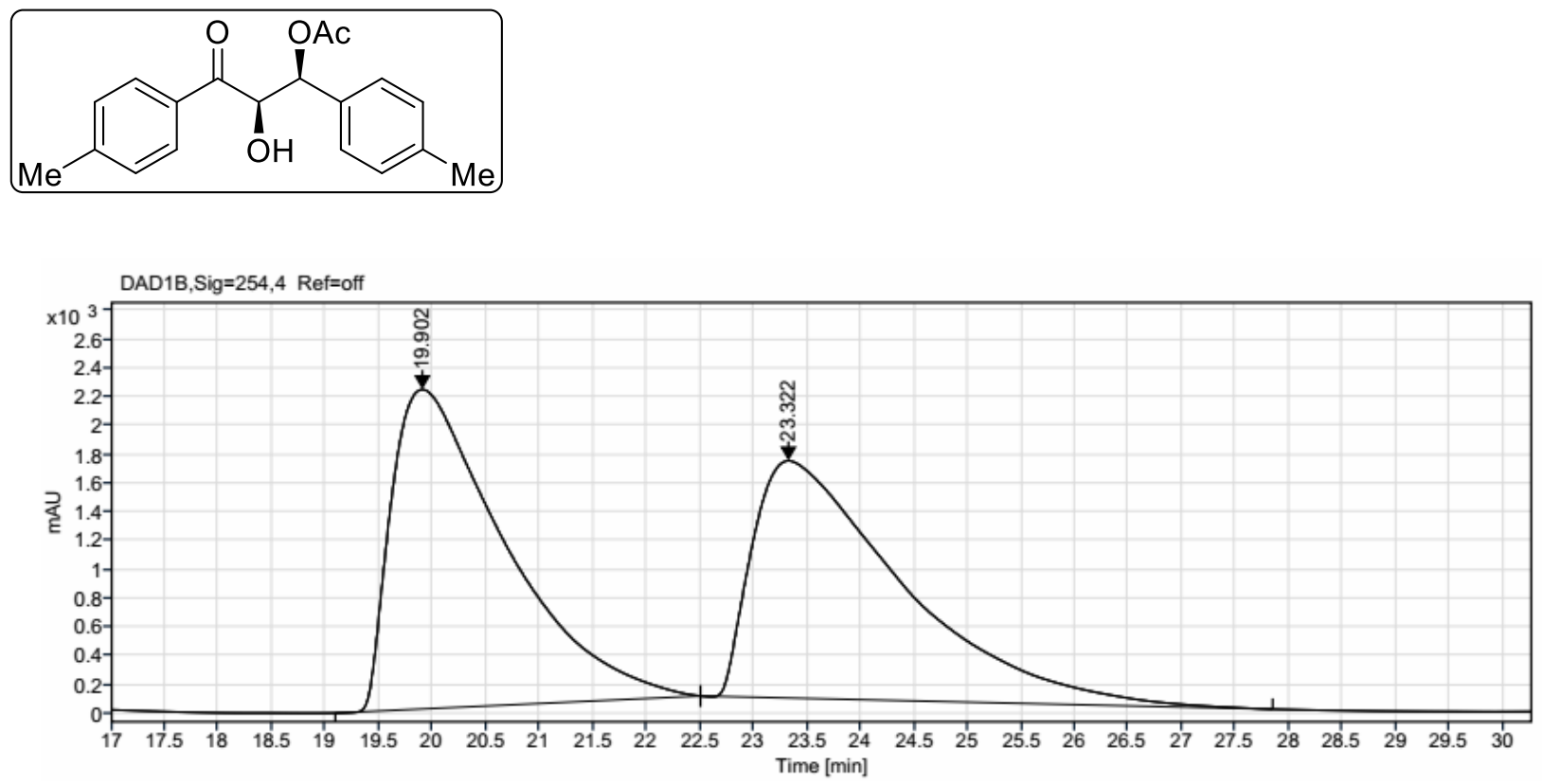

\begin{tabular}{rrrrrr} 
Signal: & \multicolumn{2}{l}{ DAD1B,Sig=254,4 Ref=off } & & & \\
RT [min] & Type & Width [min] & Area & Height & Area\% \\
19.902 & MM m & 1.08 & 164279.25 & 2219.76 & 50.66 \\
23.322 & MM m & 1.43 & 159968.80 & 1648.27 & 49.34 \\
& & Sum & 324248.06 & &
\end{tabular}

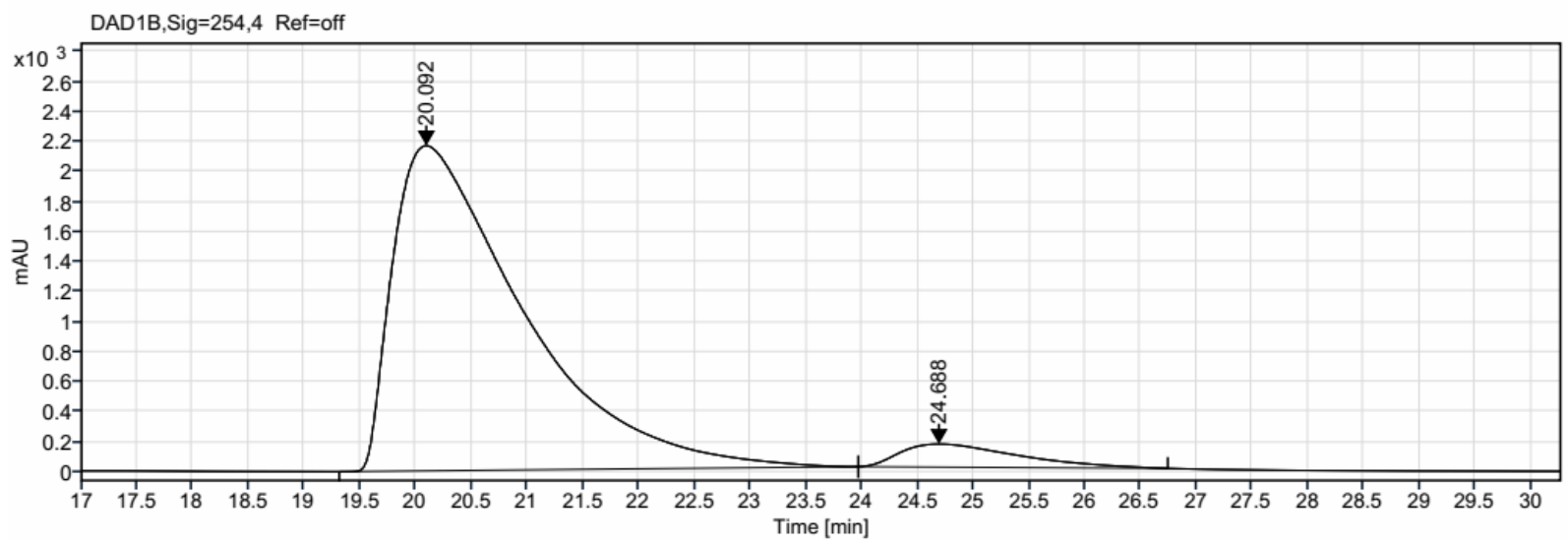

$\begin{array}{rrrrrr}\begin{array}{l}\text { Signal: } \\ \text { RT [min] }\end{array} & \text { Type } & \text { Width [min] } & \text { Area } & \text { Height } & \text { Area\% } \\ 20.092 & \text { MM m } & 1.22 & 175994.91 & 2166.91 & 93.99 \\ 24.688 & \text { MM m } & 1.10 & 11246.43 & 153.58 & 6.01 \\ & & \text { Sum } & 187241.34 & & \end{array}$



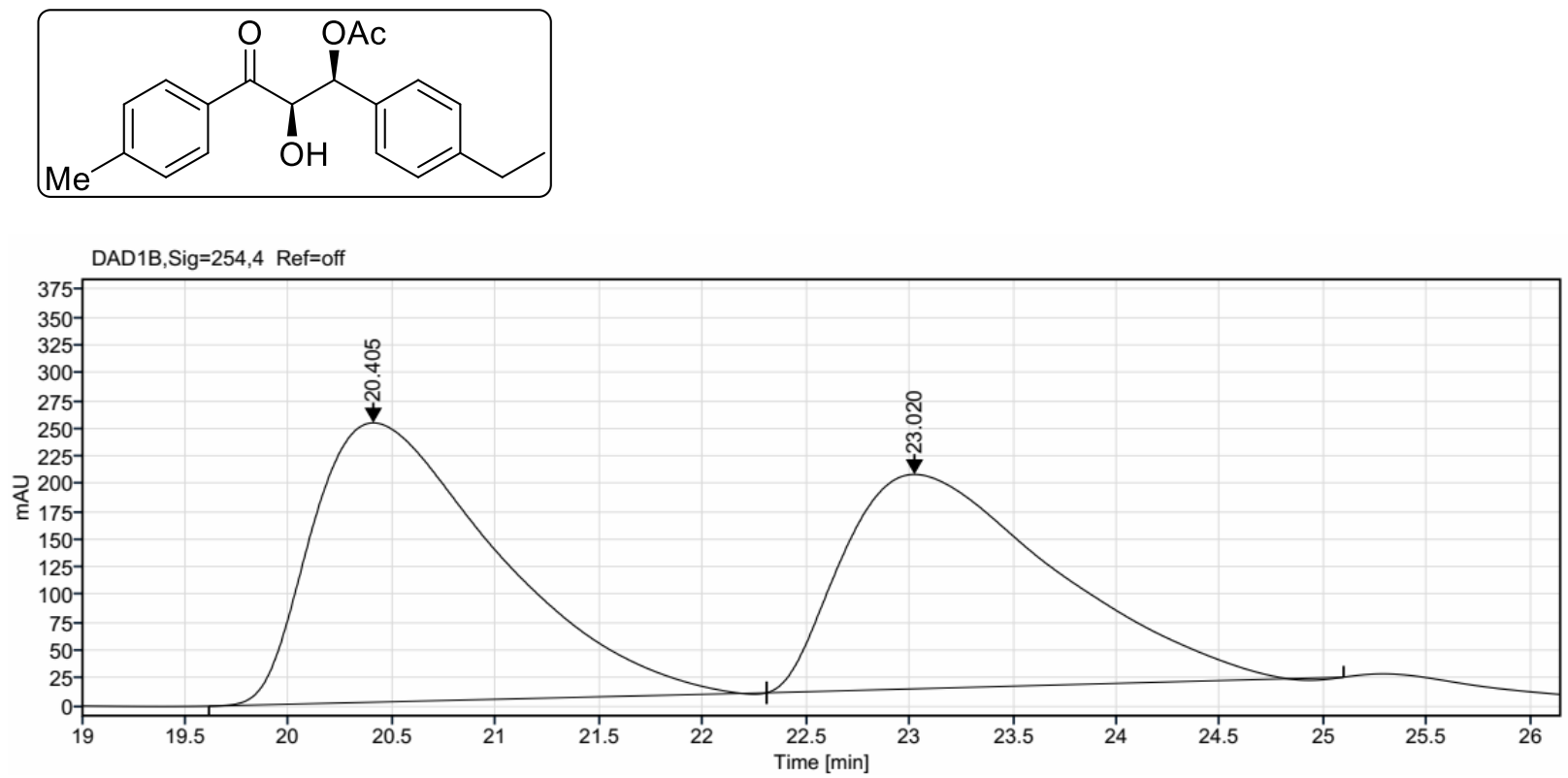

\begin{tabular}{rrrrrr} 
Signal: & \multicolumn{2}{c}{ DAD1B,Sig=254,4 Ref=off } & & \\
RT [min] & Type & Width [min] & Area & Height & Area\% \\
20.405 & MM m & 0.94 & 15663.77 & 251.77 & 53.25 \\
23.020 & MM m & 1.07 & 13750.21 & 193.19 & 46.75 \\
& & Sum & 29413.98 & &
\end{tabular}

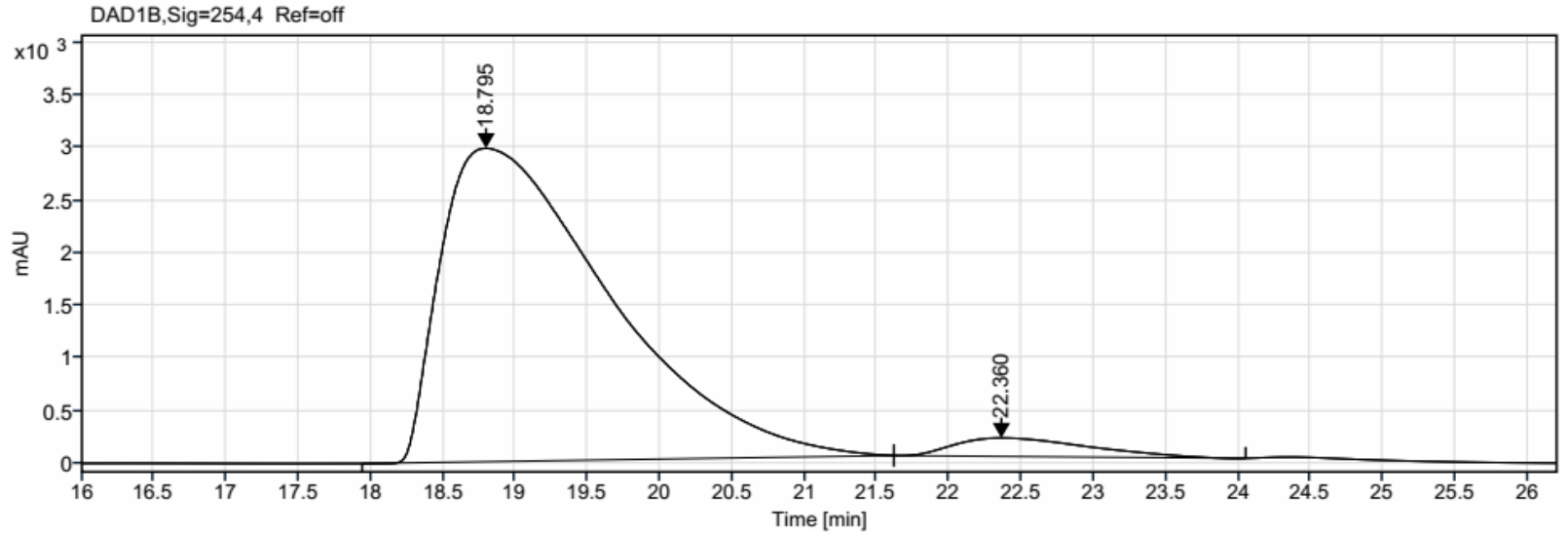

Signal: DAD1B,Sig=254,4 Ref=off

\begin{tabular}{|c|c|c|c|c|c|c|}
\hline RT [min] & Type & Width [min] & Area & Height & Area\% & Name \\
\hline 18.795 & MM m & 1.10 & 242047.25 & 2976.46 & 95.55 & \\
\hline \multirow[t]{2}{*}{22.360} & MM m & 1.02 & 11276.88 & 175.01 & 4.45 & \\
\hline & & Sum & 253324.13 & & & \\
\hline
\end{tabular}



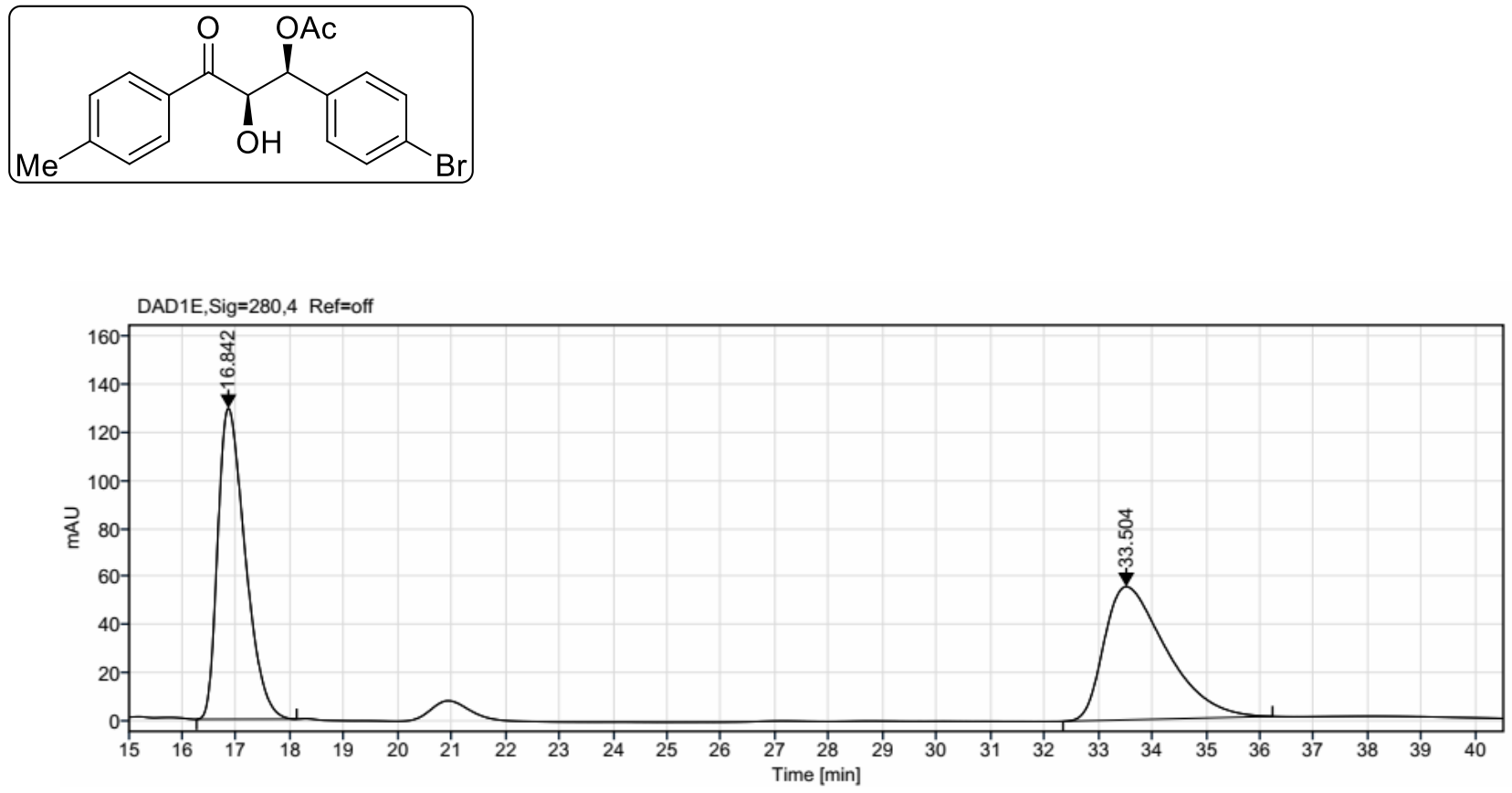

Signal: $\quad$ DAD1E, Sig $=280,4$ Ref $=$ off

\begin{tabular}{|c|c|c|c|c|c|c|}
\hline RT [min] & Type & Width [min] & Area & Height & Area\% & Name \\
\hline 16.842 & MM m & 0.55 & 4579.94 & 129.40 & 50.66 & \\
\hline \multirow[t]{2}{*}{33.504} & MM m & 1.17 & 4460.03 & 55.45 & 49.34 & \\
\hline & & Sum & 9039.97 & & & \\
\hline
\end{tabular}

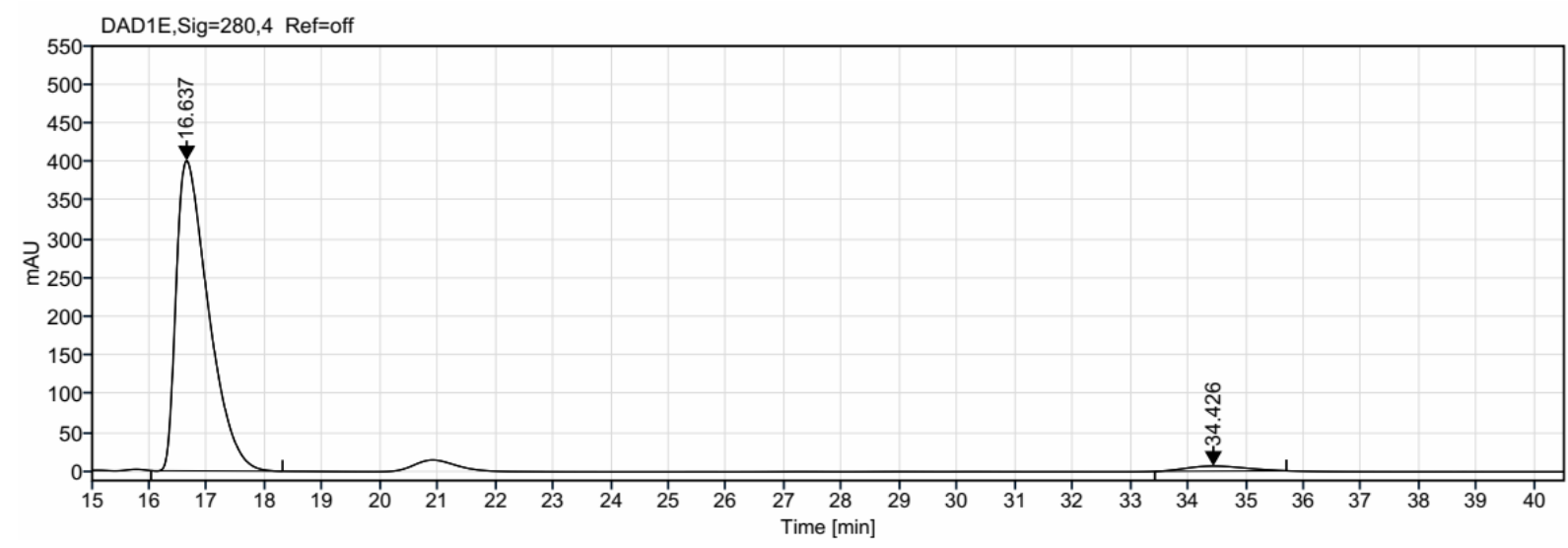

Signal: $\quad$ DAD1E, Sig $=280,4$ Ref $=$ off

$\begin{array}{rrrrrr}\text { RT [min] } & \text { Type } & \text { Width [min] } & \text { Area } & \text { Height } & \text { Area\% } \\ 16.637 & \text { MM m } & 0.59 & 15530.27 & 400.50 & 97.12 \\ 34.426 & \text { MM m } & 0.98 & 459.90 & 6.52 & 2.88\end{array}$



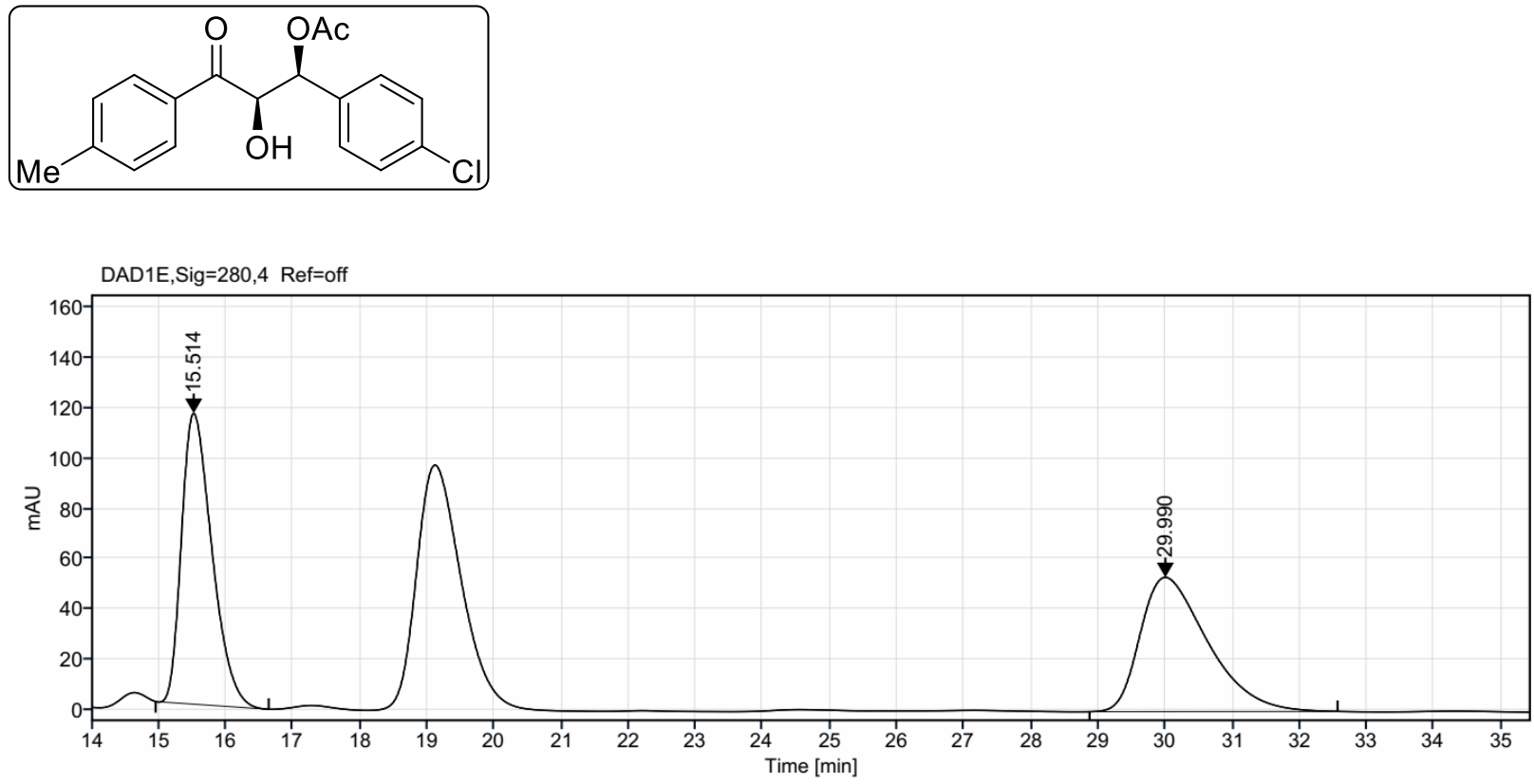

\begin{tabular}{rrrrrr} 
Signal: & \multicolumn{2}{l}{ DAD1E,Sig=280,4 Ref=off } & & & \\
RT [min] & Type & Width [min] & Area & Height & Area\% \\
15.514 & MM m & 0.49 & 3648.32 & 115.77 & 49.52 \\
29.990 & MM m & 1.06 & 3719.21 & 53.43 & 50.48 \\
& & Sum & 7367.53 & &
\end{tabular}

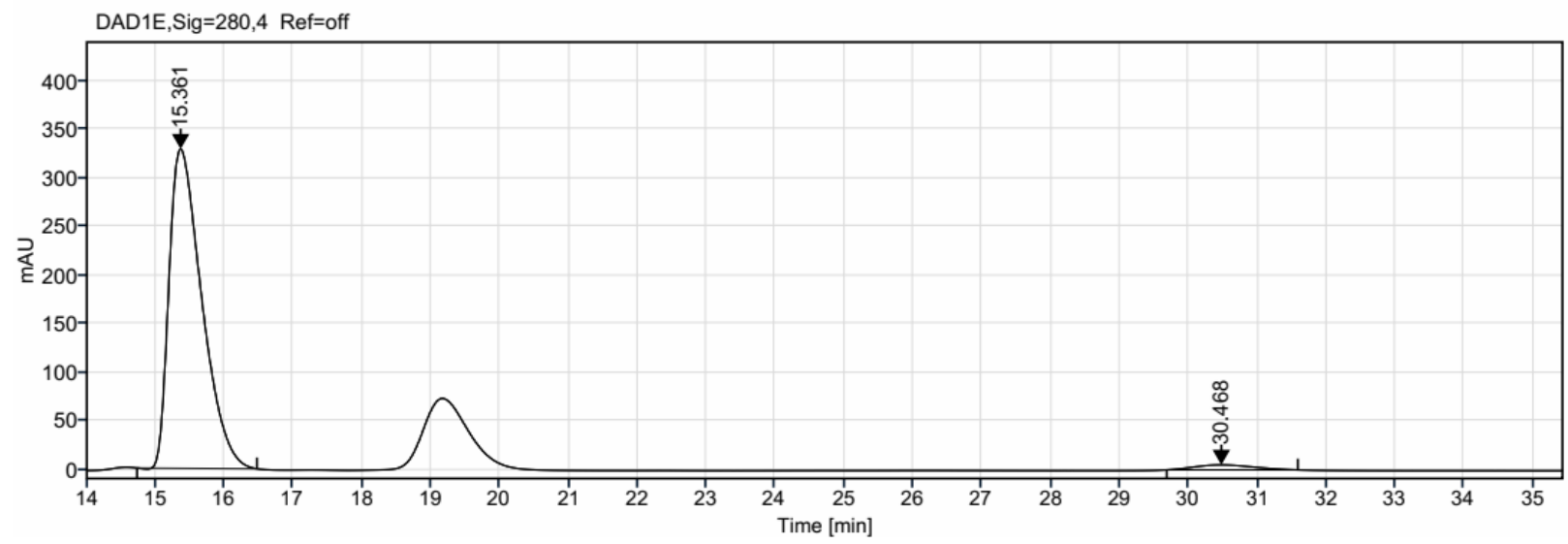

\begin{tabular}{rrrrrr} 
Signal: & \multicolumn{2}{l}{ DAD1E,Sig=280,4 Ref=off } & & & \\
RT [min] & Type & Width [min] & Area & Height & Area\% \\
15.361 & MM m & 0.51 & 10836.14 & 328.64 & 97.27 \\
30.468 & MM m & 0.78 & 303.83 & 5.16 & 2.73 \\
& & Sum & 11139.97 & &
\end{tabular}


<smiles>COC(c1cccc(C)c1)[C@H](O)[C@@H](O)C(=O)c1ccc(Br)cc1</smiles>

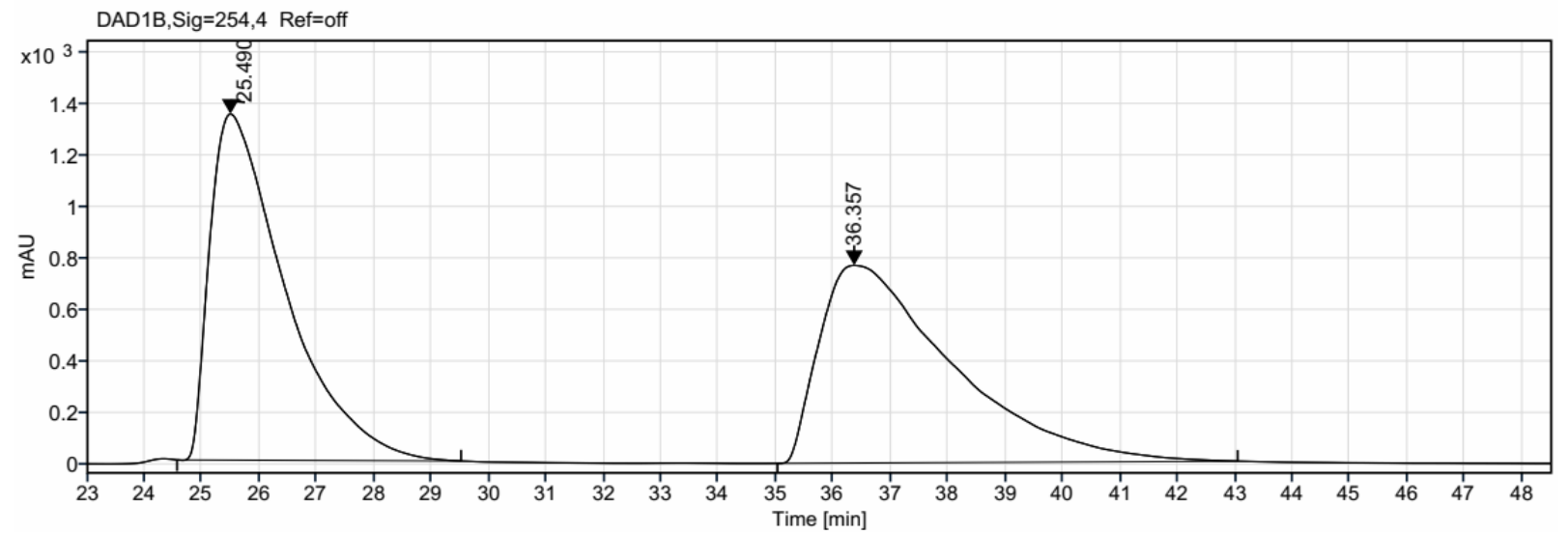

Signal: DAD1B,Sig=254,4 Ref=off

\begin{tabular}{|c|c|c|c|c|c|c|}
\hline RT [min] & Type & Width [min] & Area & Height & Area $\%$ & Name \\
\hline 25.490 & MM m & 1.37 & 123069.64 & 1346.20 & 49.89 & \\
\hline \multirow[t]{2}{*}{36.357} & MM m & 2.19 & 123632.07 & 768.63 & 50.11 & \\
\hline & & Sum & 246701.71 & & & \\
\hline
\end{tabular}

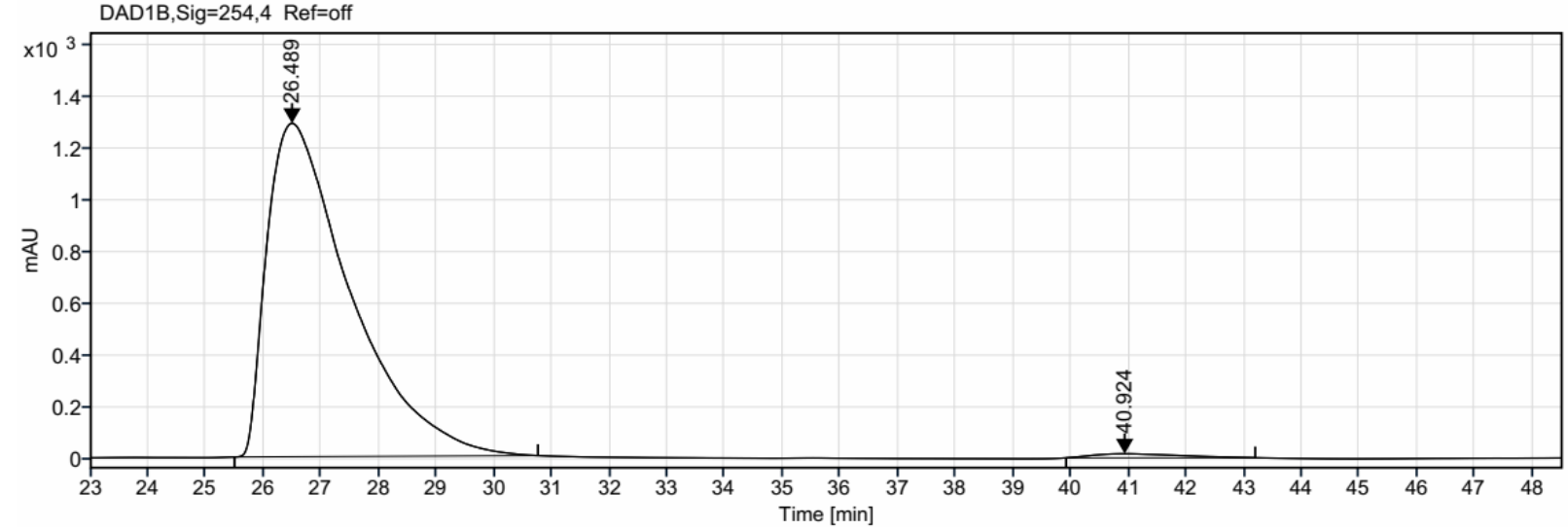

Signal: $\quad$ DAD1B,Sig $=254,4$ Ref $=$ off

$\begin{array}{rrrrrr}\text { RT [min] } & \text { Type } & \text { Width [min] } & \text { Area } & \text { Height } & \text { Area\% } \\ 26.489 & \text { MM m } & 1.46 & 129945.43 & 1289.98 & 98.77 \\ 40.924 & \text { MM m } & 1.38 & 1617.01 & 15.58 & 1.23\end{array}$



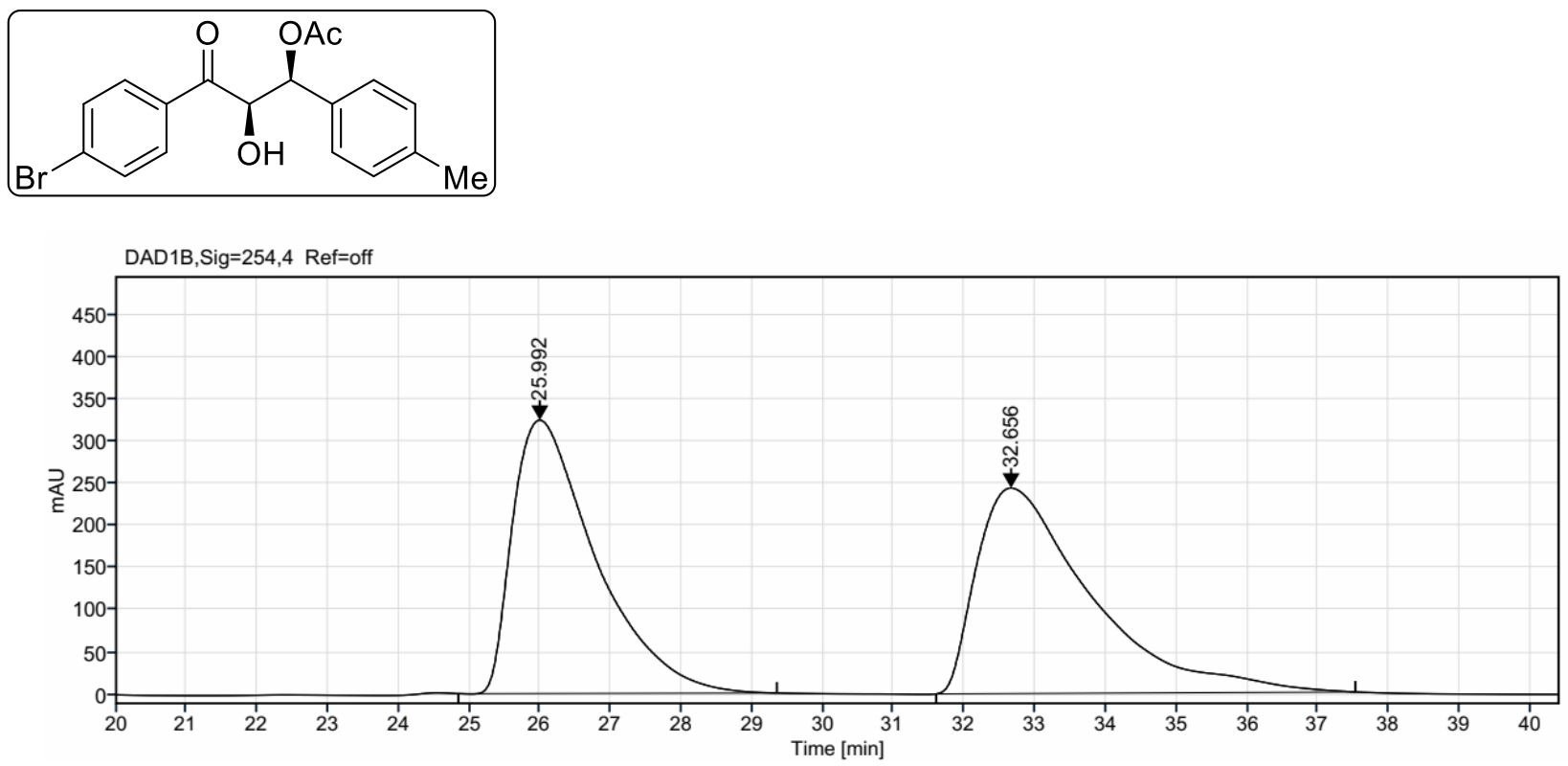

Signal: DAD1B,Sig $=254,4$ Ref $=$ off

$\begin{array}{rrrrrrr}\text { RT [min] } & \text { Type } & \text { Width [min] } & \text { Area } & \text { Height } & \text { Area\% } & \text { Name } \\ 25.992 & \text { MM m } & 1.22 & 26353.71 & 323.86 & 49.07 \\ 32.656 & \text { MM m } & 1.64 & 27351.50 & 243.51 & 50.93 \\ & & \text { Sum } & 53705.22 & & \end{array}$

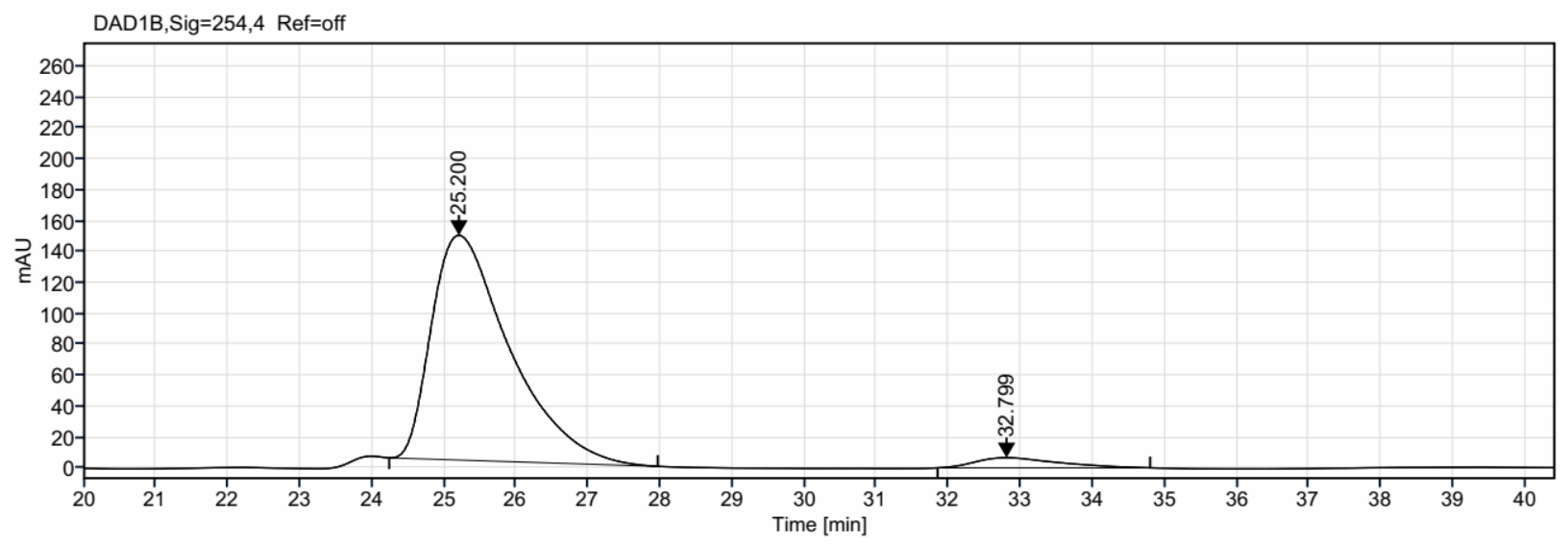

Signal: DAD1B,Sig=254,4 Ref=off

\begin{tabular}{|c|c|c|c|c|c|c|}
\hline RT [min] & Type & Width [min] & Area & Height & Area $\%$ & Name \\
\hline 25.200 & $\mathrm{MM} \mathrm{m}$ & 1.12 & 10962.23 & 145.28 & 95.43 & \\
\hline \multirow[t]{2}{*}{32.799} & MM m & 0.97 & 524.57 & 6.58 & 4.57 & \\
\hline & & Sum & 11486.79 & & & \\
\hline
\end{tabular}



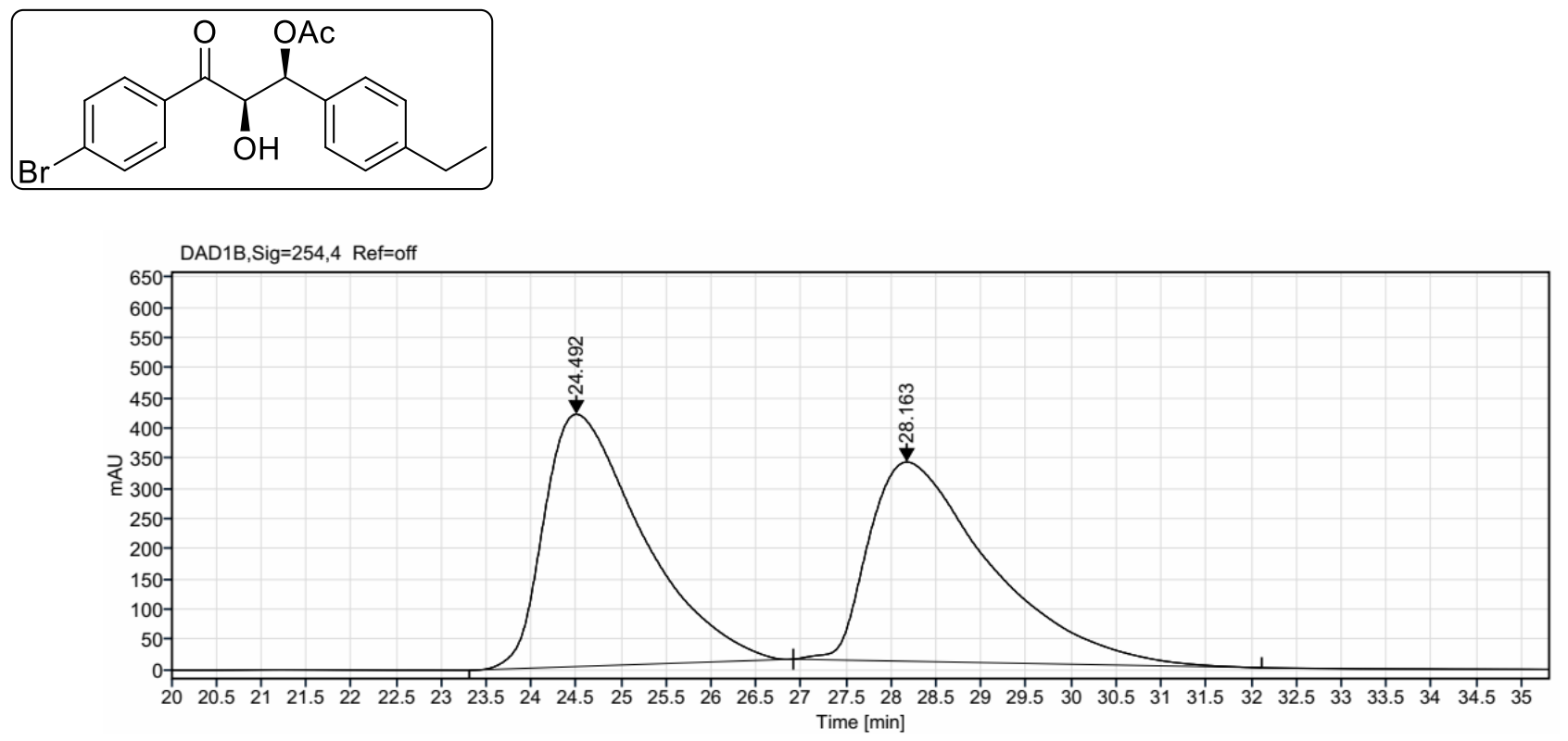

Signal: DAD1B,Sig=254,4 Ref=off

\begin{tabular}{|c|c|c|c|c|c|c|}
\hline RT [min] & Type & Width [min] & Area & Height & Area\% & Name \\
\hline 24.492 & MM m & 1.13 & 31829.27 & 418.06 & 50.69 & \\
\hline \multirow[t]{2}{*}{28.163} & MM m & 1.39 & 30966.04 & 329.94 & 49.31 & \\
\hline & & Sum & 62795.30 & & & \\
\hline
\end{tabular}

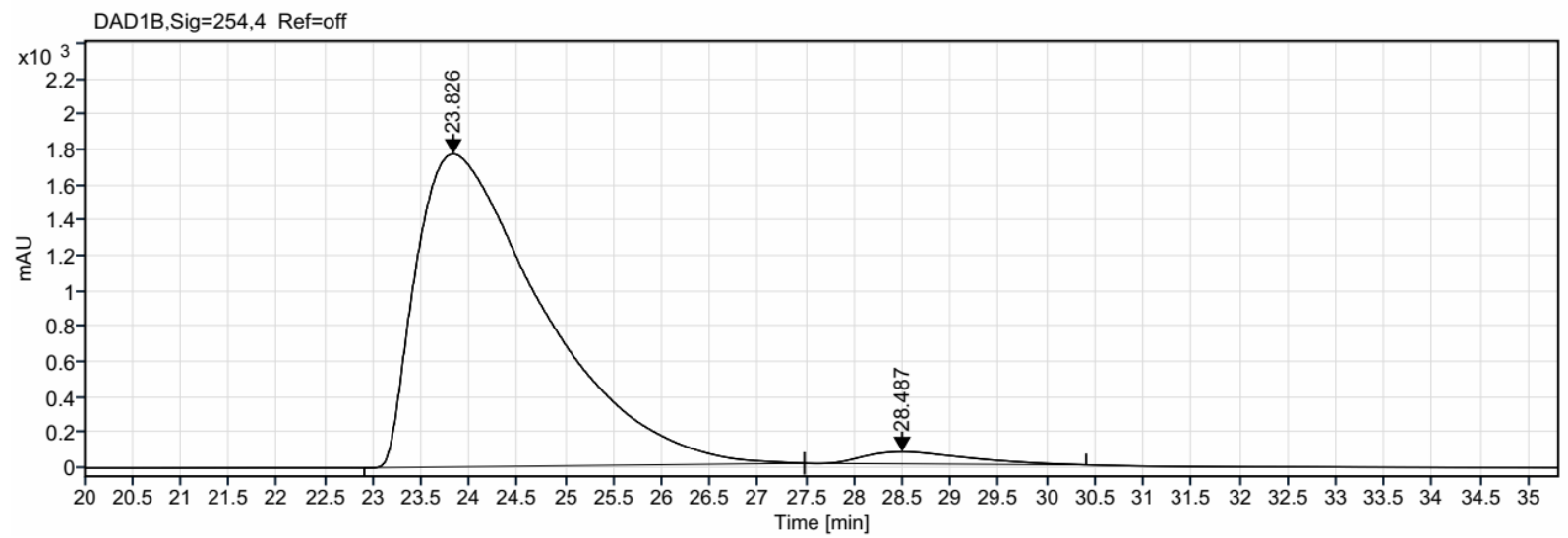

Signal: DAD1B,Sig $=254,4$ Ref $=$ off

$\begin{array}{rrrrrr}\text { RT [min] } & \text { Type } & \text { Width [min] } & \text { Area } & \text { Height } & \text { Area\% } \\ 23.826 & \text { MM m } & 1.39 & 162392.76 & 1772.57 & 96.96 \\ 28.487 & \text { MM m } & 1.10 & 5090.86 & 68.45 & 3.04\end{array}$



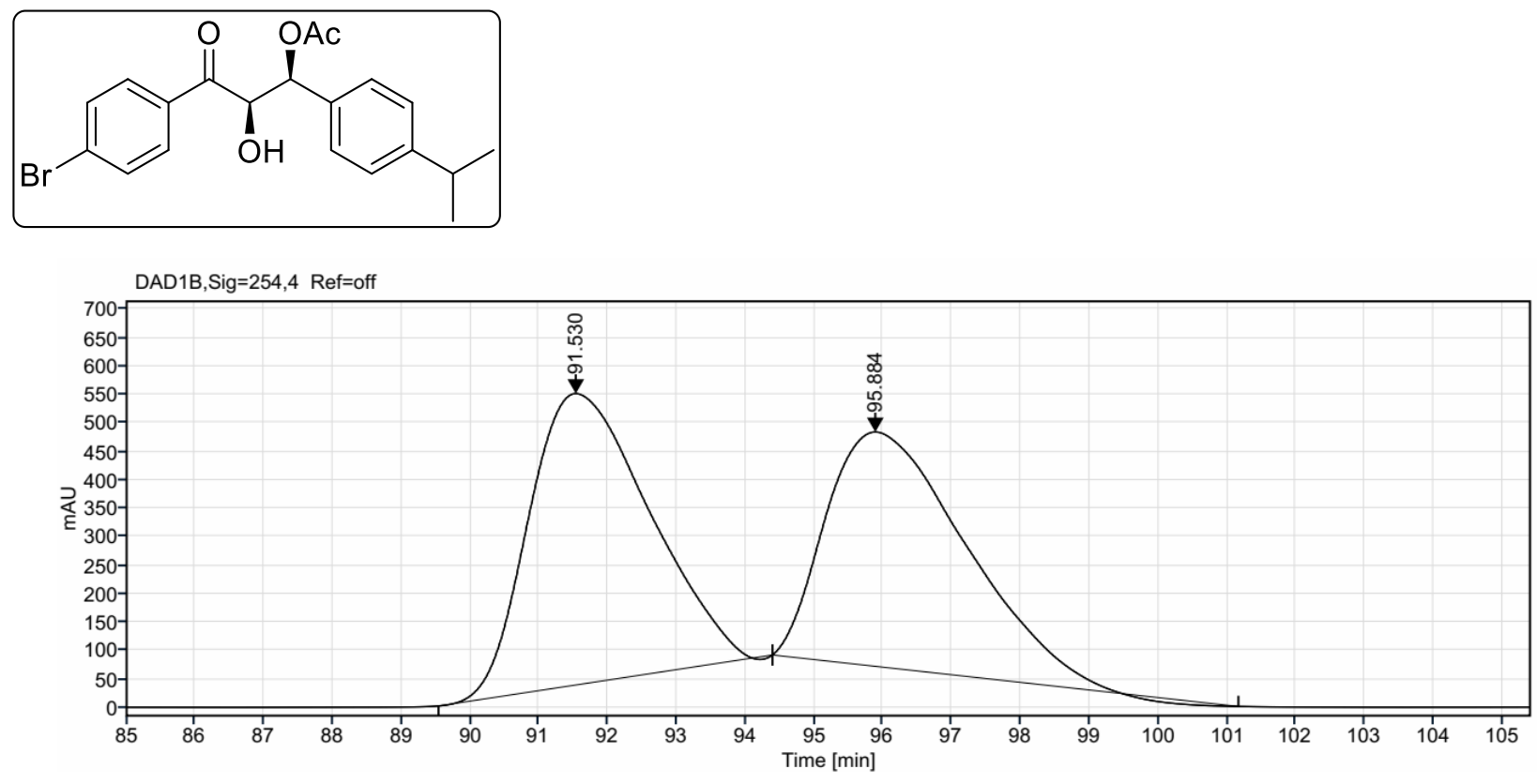

\begin{tabular}{rrrrrr} 
Signal: & \multicolumn{2}{c}{ DAD1B,Sig=254,4 Ref=off } & & & \\
RT [min] & Type & Width [min] & Area & Height & Area\% \\
91.530 & MM m & 1.88 & 62164.19 & 512.78 & 51.63 \\
95.884 & MM m & 2.16 & 58231.93 & 412.34 & 48.37 \\
& & Sum & 120396.13 & &
\end{tabular}

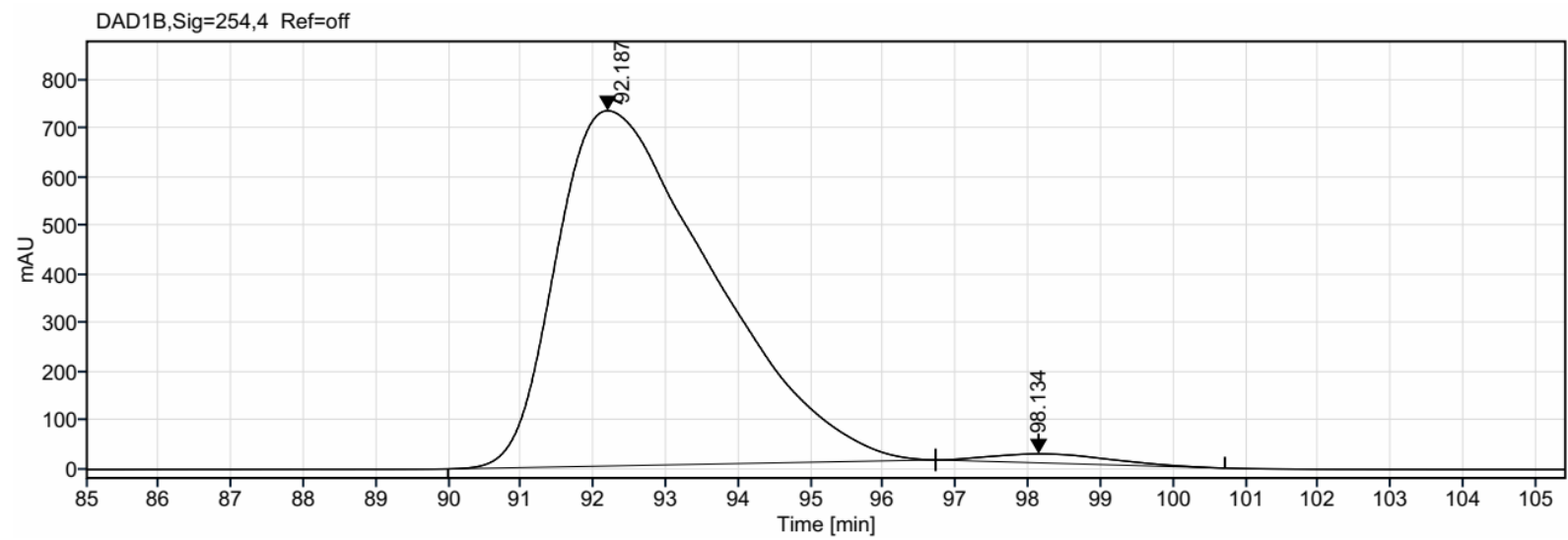

Signal: DAD1B,Sig $=254,4$ Ref $=$ off

$\begin{array}{rrrrrr}\text { RT [min] } & \text { Type } & \text { Width [min] } & \text { Area } & \text { Height } & \text { Area\% } \\ 92.187 & \text { MM m } & 2.20 & 109353.96 & 729.79 & 98.09 \\ 98.134 & \text { MM m } & 1.42 & 2131.54 & 18.37 & 1.91\end{array}$


<smiles>CCOC(C(C)=O)[C@H](O)[C@@H](O)C(=O)c1ccc(Cl)cc1</smiles>

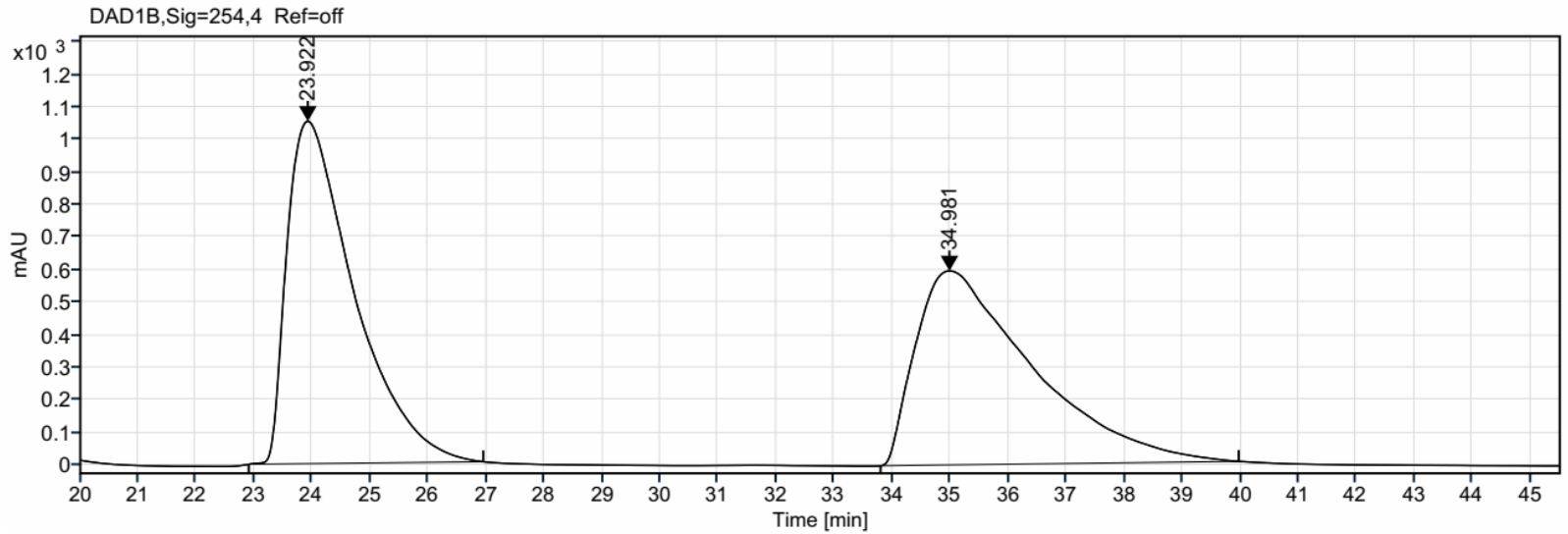

Signal: $\quad$ DAD1B,Sig $=254,4$ Ref $=$ off

\begin{tabular}{|c|c|c|c|c|c|c|}
\hline RT [min] & Type & Width [min] & Area & Height & Area $\%$ & Name \\
\hline 23.922 & MM m & 1.18 & 84693.43 & 1053.71 & 50.44 & \\
\hline \multirow[t]{2}{*}{34.981} & MM m & 1.91 & 83204.22 & 597.31 & 49.56 & \\
\hline & & Sum & 167897.65 & & & \\
\hline
\end{tabular}

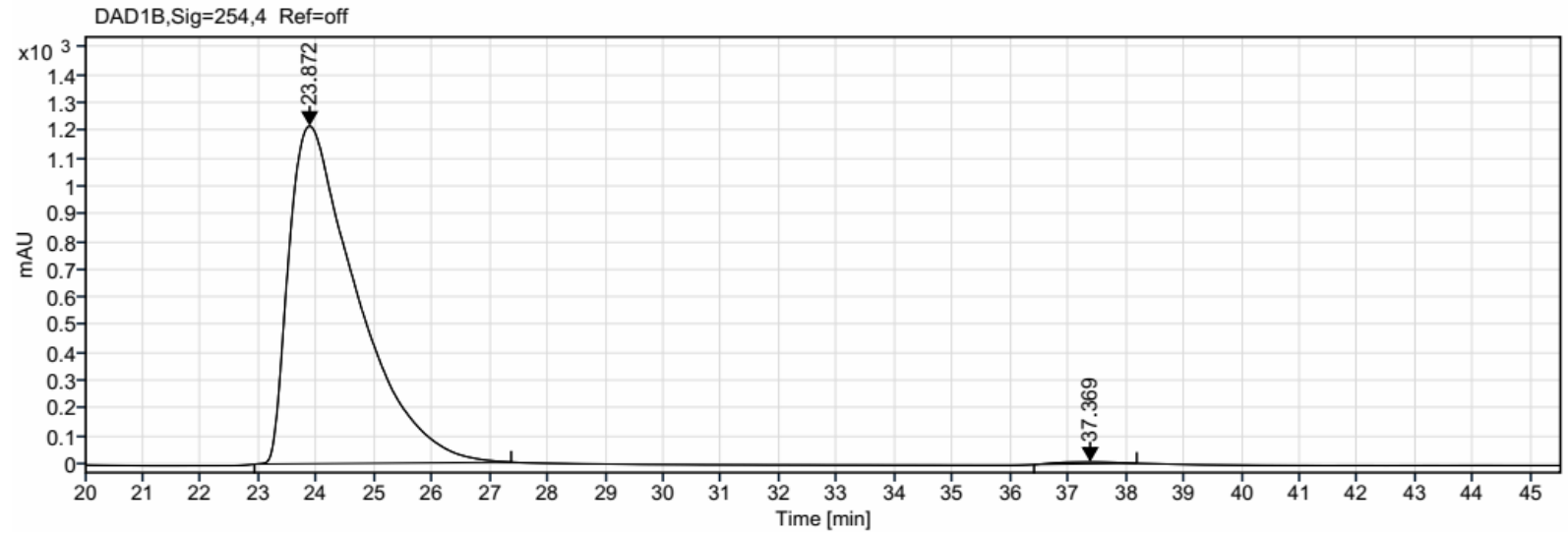

Signal: $\quad$ DAD1B, Sig $=254,4$ Ref $=$ off

\begin{tabular}{|c|c|c|c|c|c|c|}
\hline RT [min] & Type & Width [min] & Area & Height & Area\% & Name \\
\hline 23.872 & MM m & 1.19 & 99632.64 & 1215.85 & 99.51 & \\
\hline \multirow[t]{2}{*}{37.369} & MM m & 0.86 & 486.93 & 6.86 & 0.49 & \\
\hline & & Sum & 100119.57 & & & \\
\hline
\end{tabular}



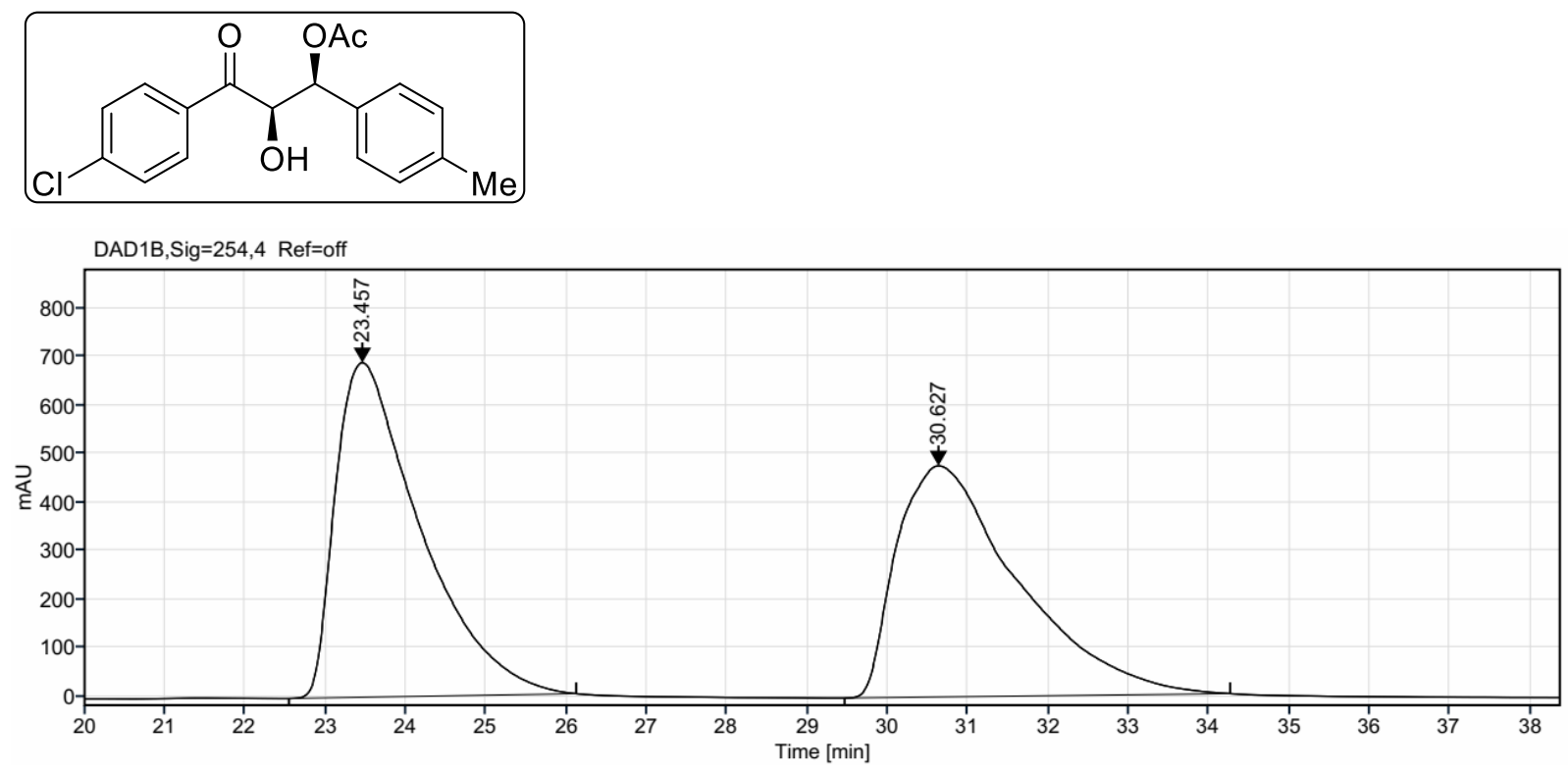

\begin{tabular}{rrrrrr}
\multicolumn{1}{l}{$\begin{array}{l}\text { Signal: } \\
\text { RT [min] }\end{array}$} & Type & Width [min] & Area & Height & Area\% \\
23.457 & MM m & 1.06 & 50547.21 & 688.97 & 50.39 \\
30.627 & MM m & 1.44 & 49766.87 & 476.09 & 49.61
\end{tabular}

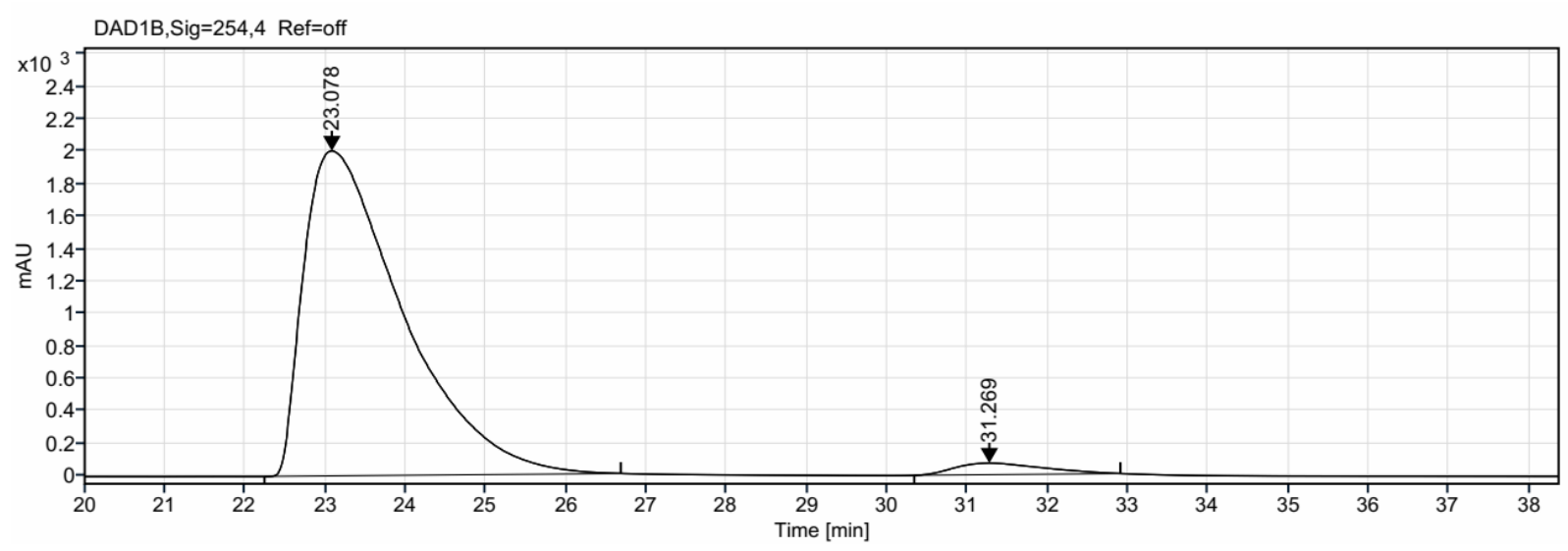

\begin{tabular}{rrrrrr} 
Signal: & \multicolumn{2}{c}{ DAD1B,Sig=254,4 Ref=off } & & \\
RT [min] & Type & Width [min] & Area & Height & Area\% \\
23.078 & MM m & 1.27 & 171699.01 & 2006.07 & 96.94 \\
31.269 & MM m & 1.13 & 5410.81 & 71.44 & 3.06 \\
& & Sum & 177109.83 & &
\end{tabular}



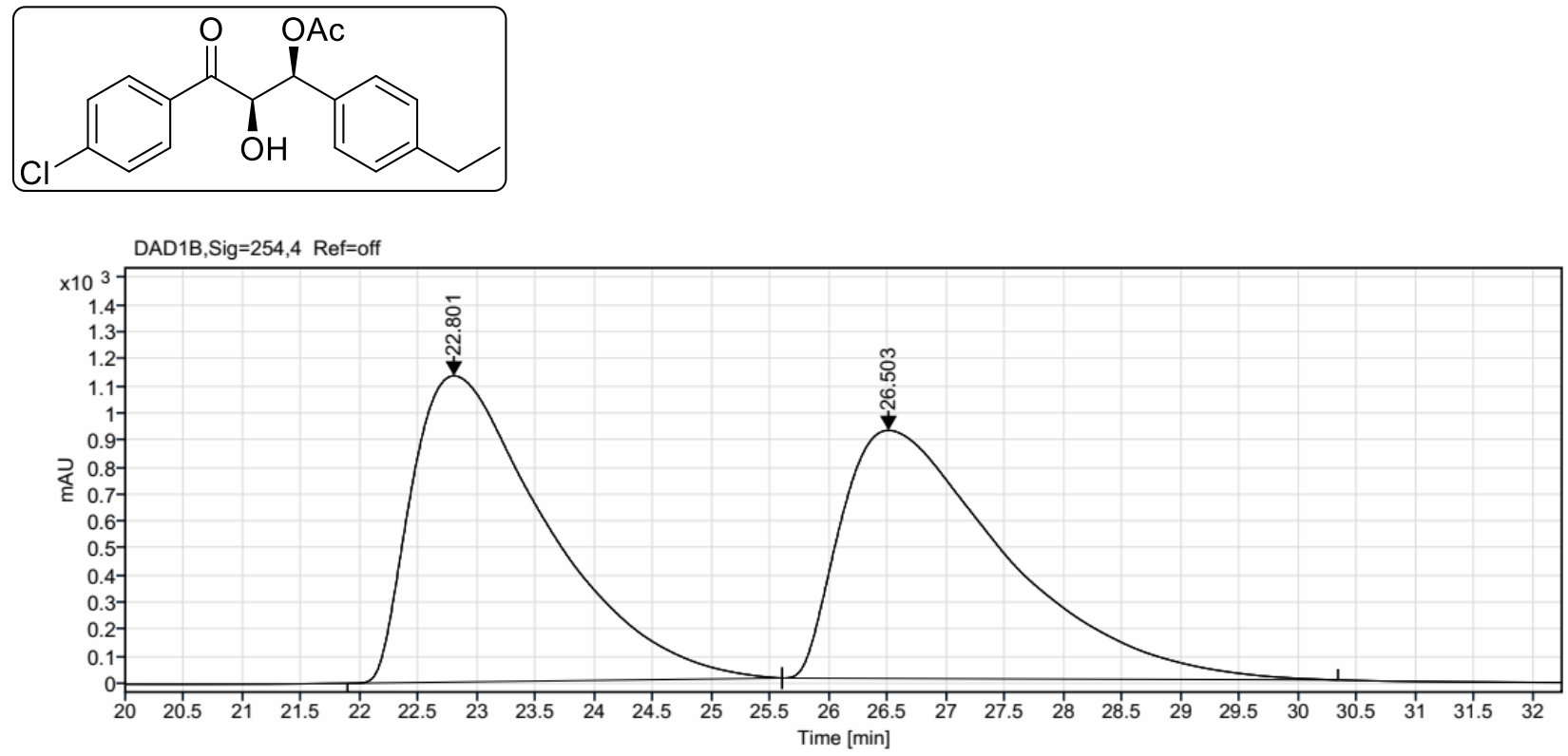

\begin{tabular}{|c|c|c|c|c|c|c|}
\hline Signal: & AD1B,S & 254,4 Ref=off & & & & \\
\hline RT [min] & Type & Width [min] & Area & Height & Area\% & Name \\
\hline 22.801 & MM m & 1.21 & 91183.64 & 1132.91 & 50.53 & \\
\hline 26.503 & MM m & 1.42 & 89286.42 & 917.60 & 49.47 & \\
\hline & & Sum & 180470.05 & & & \\
\hline
\end{tabular}

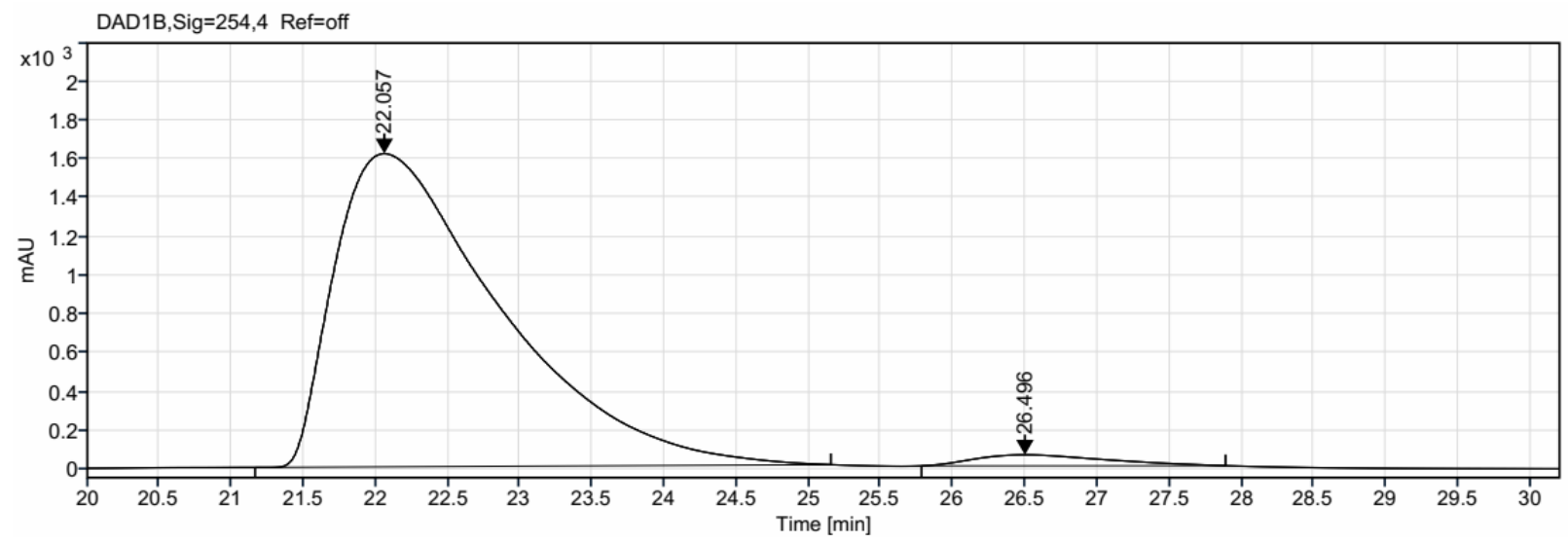

Signal: $\quad$ DAD1B,Sig=254,4 Ref=off

$\begin{array}{rrrrrrr}\text { RT [min] } & \text { Type } & \text { Width [min] } & \text { Area } & \text { Height } & \text { Area\% } & \text { Name } \\ 22.057 & \text { MM m } & 1.20 & 129223.57 & 1615.06 & 97.30 \\ 26.496 & \text { MM m } & 0.96 & 3589.63 & 56.57 & 2.70 & \\ & & \text { Sum } & 132813.20 & & \end{array}$



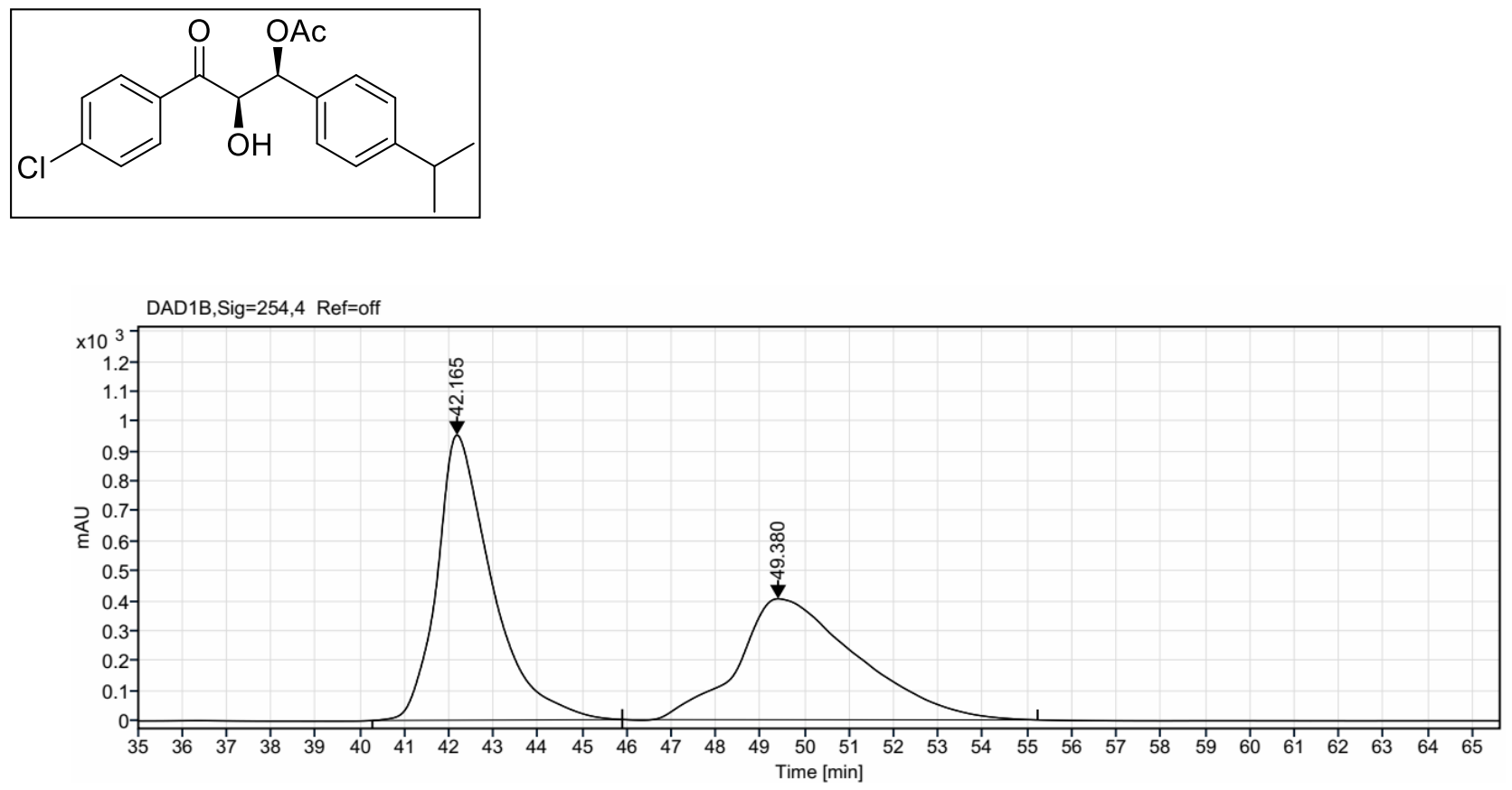

\begin{tabular}{|c|c|c|c|c|c|c|}
\hline Signal: & AD1B,S & $=254,4$ Ref $=$ off & & & & \\
\hline RT [min] & Type & Width [min] & Area & Height & Area\% & Name \\
\hline 42.165 & MM m & 1.20 & 82174.37 & 954.66 & 52.09 & \\
\hline \multirow[t]{2}{*}{49.380} & MM m & 2.42 & 75594.22 & 404.81 & 47.91 & \\
\hline & & Sum & 157768.59 & & & \\
\hline
\end{tabular}

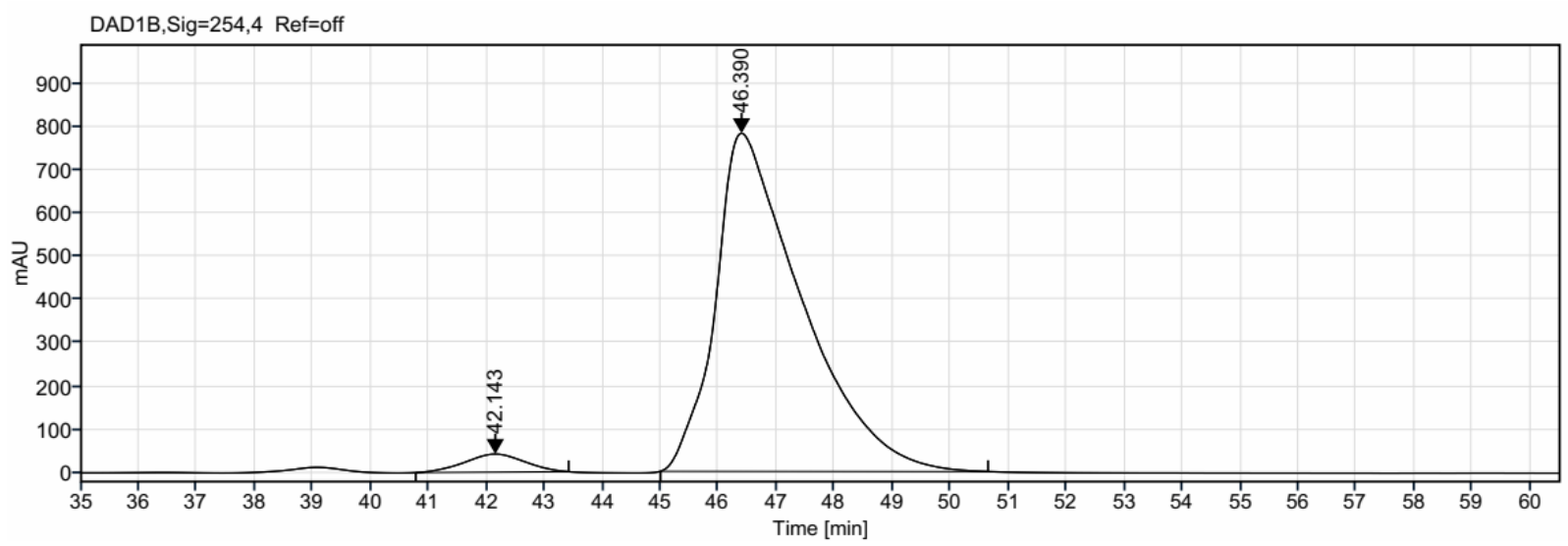

\begin{tabular}{rrrrrr} 
Signal: & \multicolumn{2}{l}{ DAD1B,Sig=254,4 Ref=off } & & & \\
RT [min] & Type & Width [min] & Area & Height & Area\% \\
42.143 & MM m & 1.07 & 3017.52 & 41.82 & 3.60 \\
46.390 & MM m & 1.40 & 80894.69 & 781.20 & 96.40 \\
& & Sum & 83912.21 & &
\end{tabular}



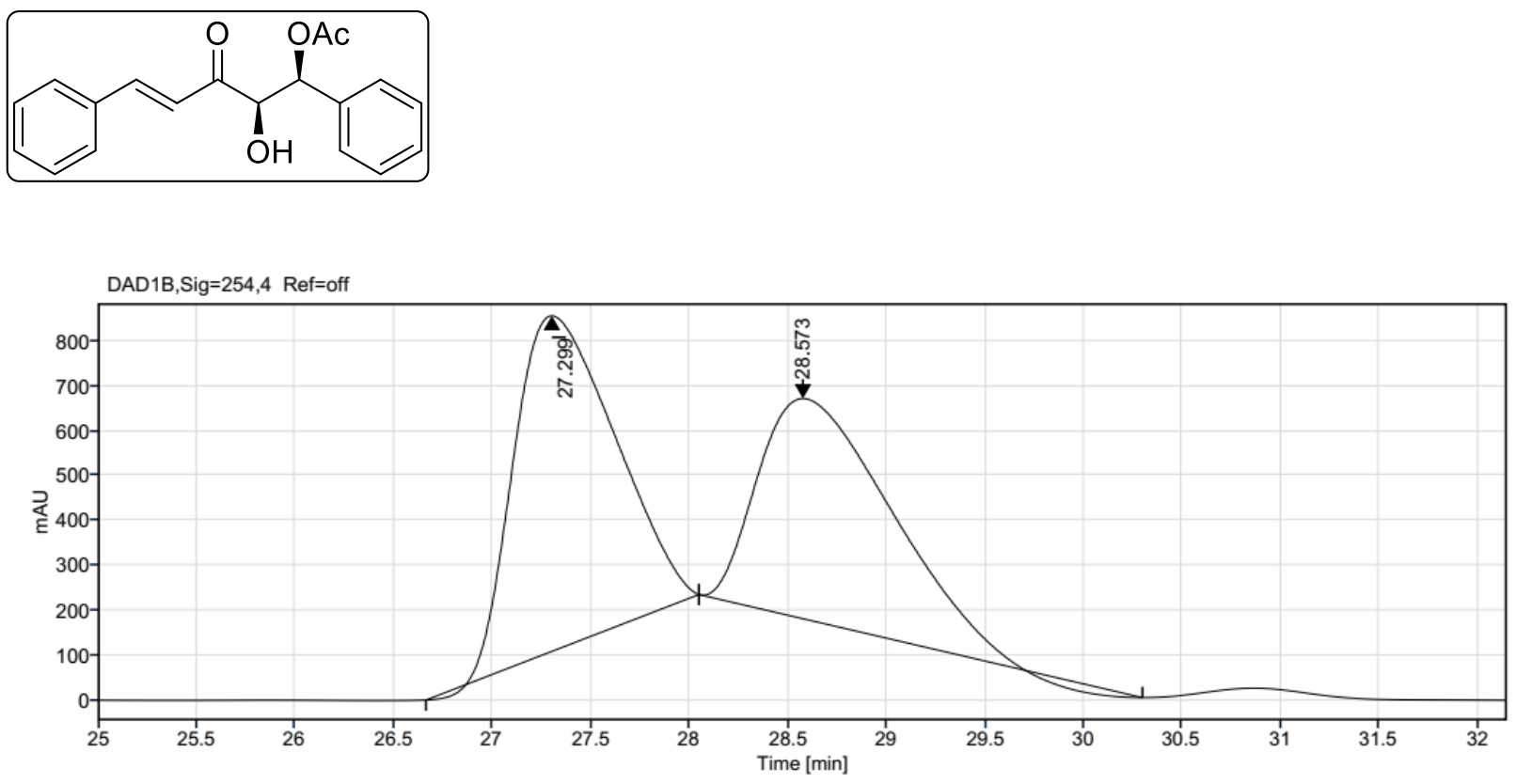

\begin{tabular}{rrrrrrr}
\multicolumn{1}{l}{$\begin{array}{l}\text { Signal: } \\
\text { RT [min] }\end{array}$} & Type & Width $[\mathrm{min}]$ & Area & Height & Area\% & Name \\
27.299 & MM m & 0.57 & 26392.91 & 746.41 & 53.45 & \\
28.573 & MM m & 0.75 & 22983.54 & 489.78 & 46.55 \\
& & Sum & 49376.45 & &
\end{tabular}

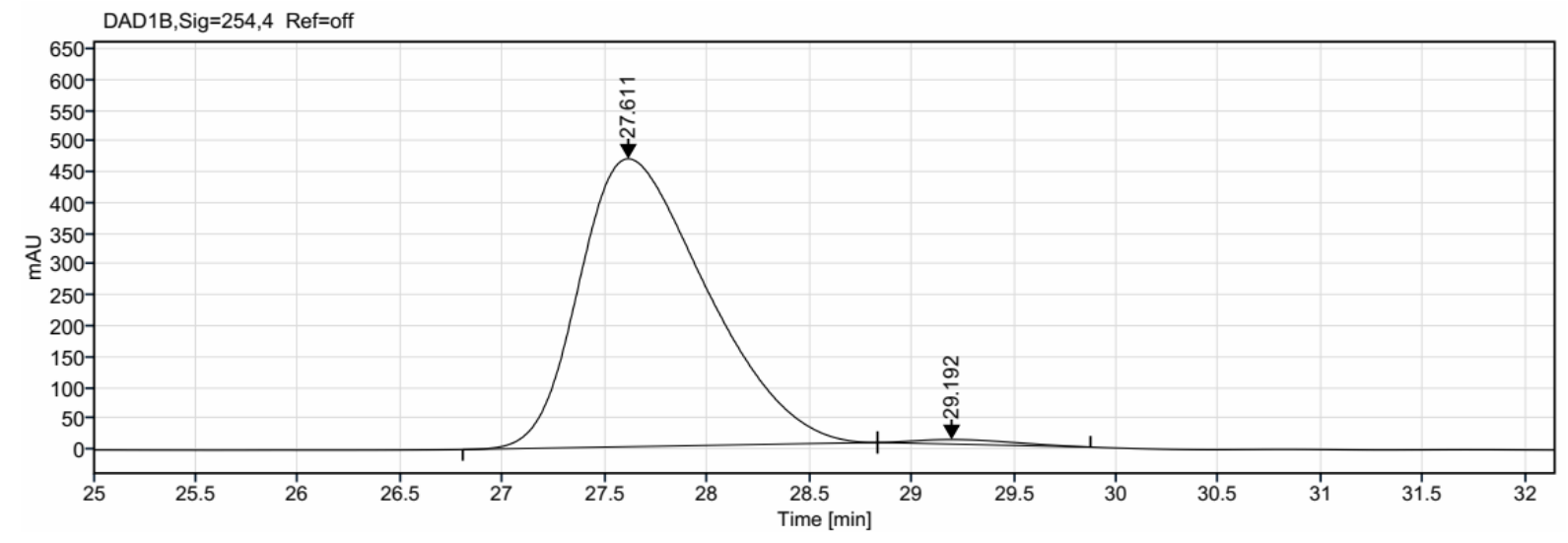

Signal: $\quad$ DAD1B,Sig $=254,4$ Ref $=$ off

\begin{tabular}{|c|c|c|c|c|c|c|}
\hline RT [min] & Type & Width [min] & Area & Height & Area $\%$ & Name \\
\hline 27.611 & $\mathrm{MM} \mathrm{m}$ & 0.67 & 20159.93 & 467.22 & 98.77 & \\
\hline \multirow[t]{2}{*}{29.192} & $\mathrm{MM} \mathrm{m}$ & 0.54 & 251.74 & 7.33 & 1.23 & \\
\hline & & Sum & 20411.67 & & & \\
\hline
\end{tabular}


<smiles>O=C(O[C@H](c1ccccc1)[C@@H](O)C(=O)c1ccccc1)C1CC2CCC1C2</smiles>

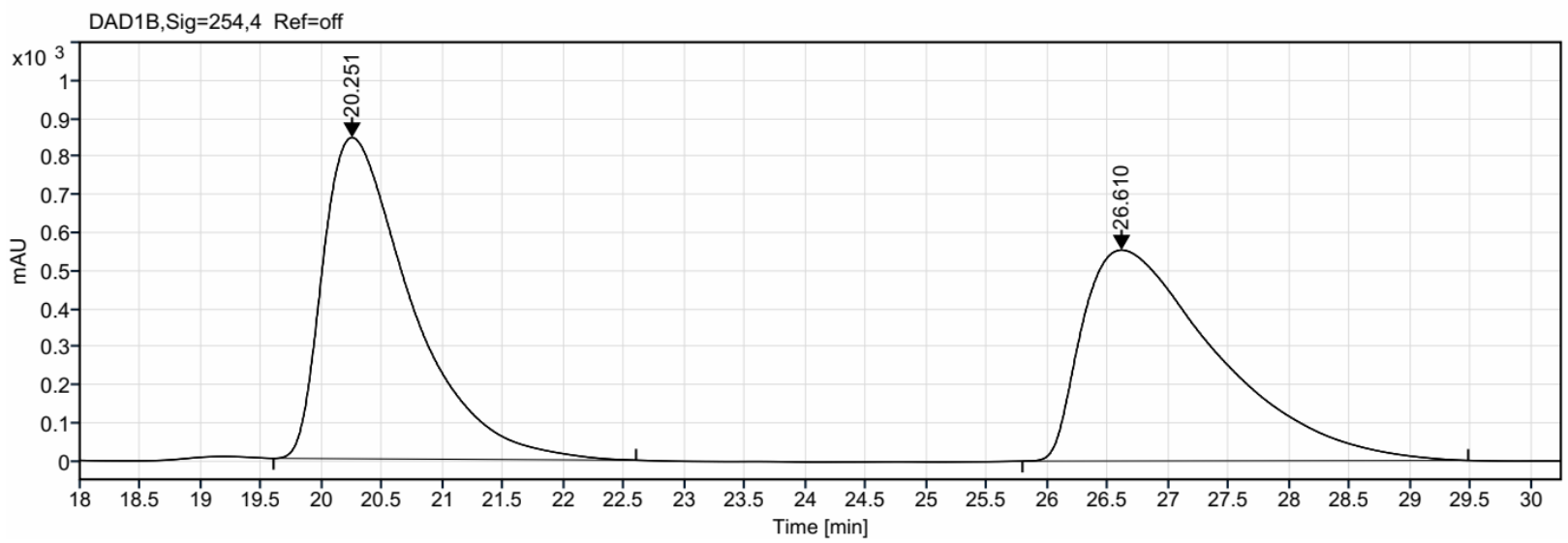

Signal: DAD1B,Sig $=254,4$ Ref=off

$\begin{array}{rrrrrr}\text { RT [min] } & \text { Type } & \text { Width [min] } & \text { Area } & \text { Height } & \text { Area\% } \\ 20.251 & \text { MM m } & 0.77 & 43018.14 & 843.23 & 50.02 \\ 26.610 & \text { MM m } & 1.17 & 42975.55 & 553.79 & 49.98\end{array}$

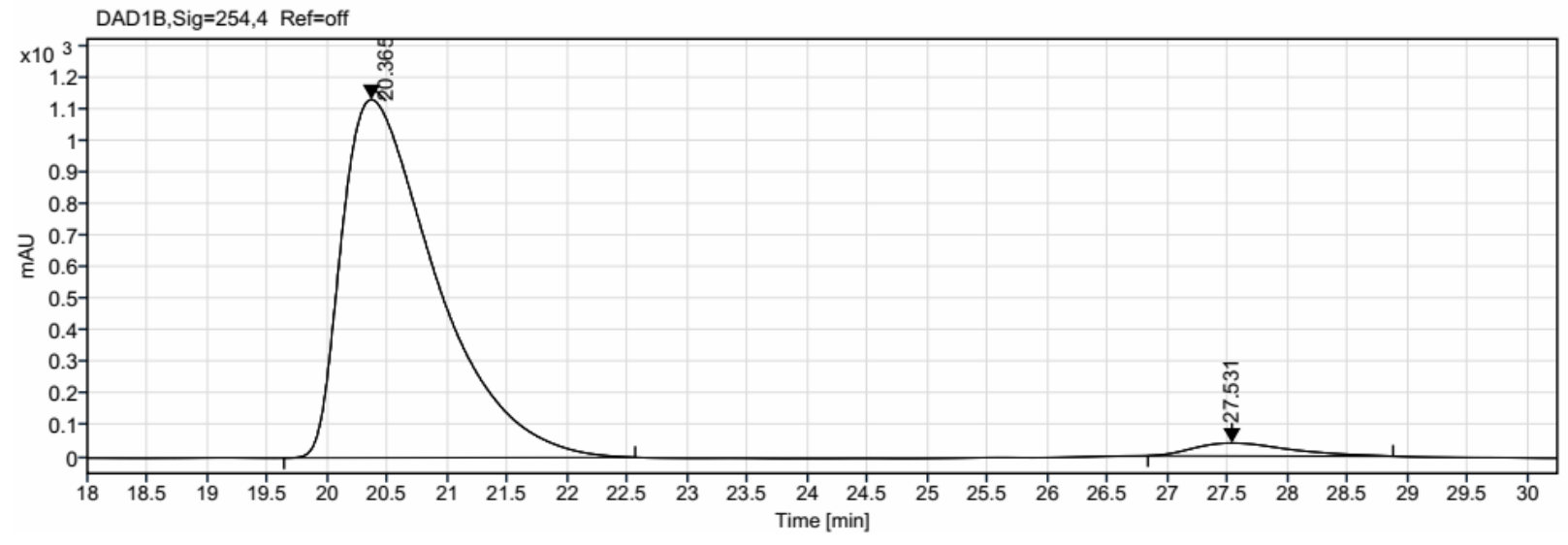

Signal: DAD1B,Sig=254,4 Ref=off

$\begin{array}{rrrrrr}\text { RT [min] } & \text { Type } & \text { Width [min] } & \text { Area } & \text { Height } & \text { Area\% } \\ 20.365 & \text { MM m } & 0.83 & 61702.63 & 1131.34 & 96.53 \\ 27.531 & \text { MM m } & 0.84 & 2221.27 & 40.47 & 3.47 \\ & & \text { Sum } & 63923.90 & & \end{array}$



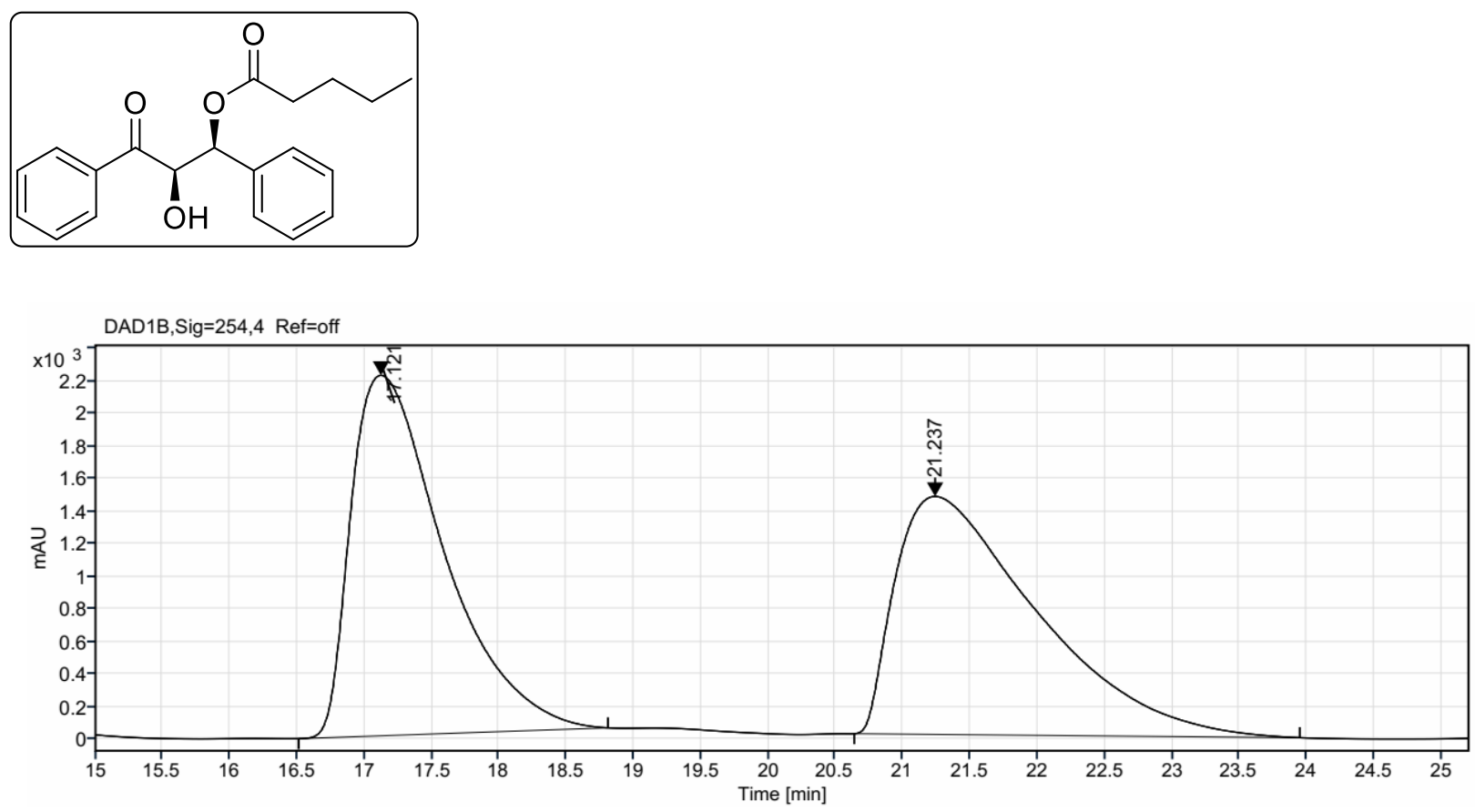

Signal: $\quad$ DAD1B,Sig $=254,4$ Ref $=$ off

$\begin{array}{rrrrrr}\text { RT [min] } & \text { Type } & \text { Width [min] } & \text { Area } & \text { Height } & \text { Area\% } \\ 17.121 & \text { MM m } & 0.73 & 106235.30 & 2214.97 & 49.83 \\ 21.237 & \text { MM m } & 1.10 & 106962.97 & 1462.41 & 50.17 \\ & & \text { Sum } & 213198.27 & & \end{array}$

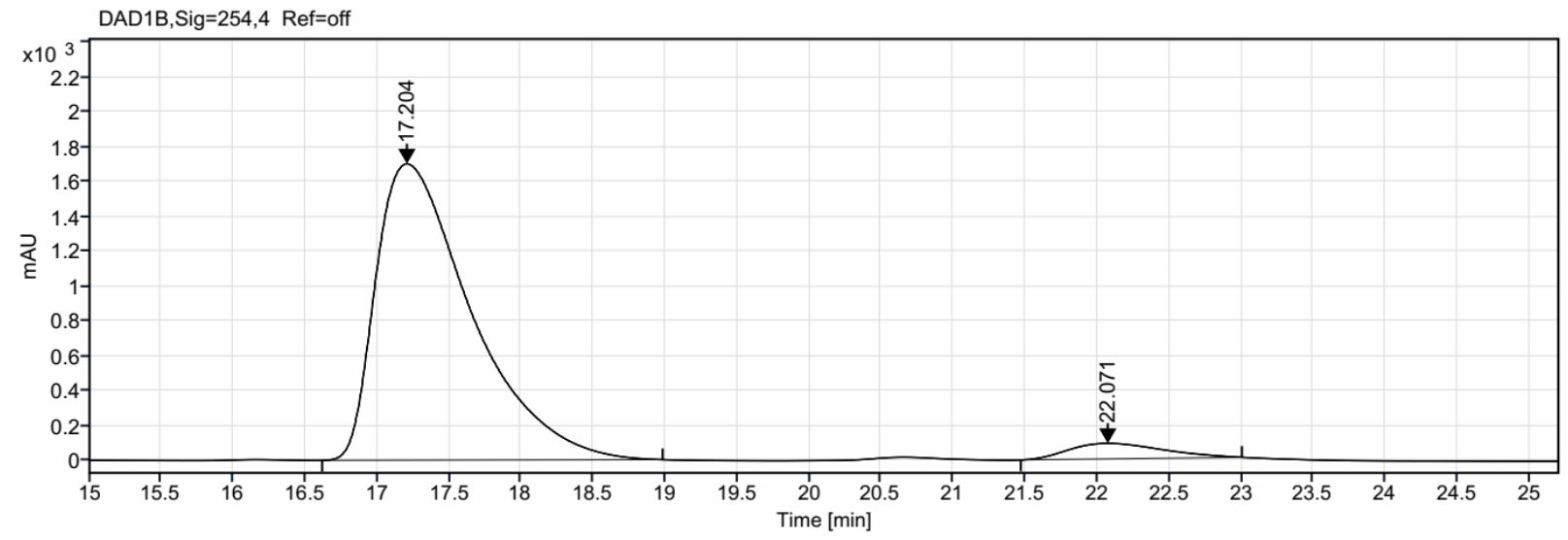

Signal: $\quad$ DAD1B,Sig=254,4 Ref=off

\begin{tabular}{|c|c|c|c|c|c|c|}
\hline RT [min] & Type & Width [min] & Area & Height & Area\% & Name \\
\hline 17.204 & MM m & 0.70 & 78239.19 & 1701.52 & 95.09 & \\
\hline \multirow[t]{2}{*}{22.071} & MM m & 0.72 & 4035.58 & 89.30 & 4.91 & \\
\hline & & Sum & 82274.77 & & & \\
\hline
\end{tabular}


